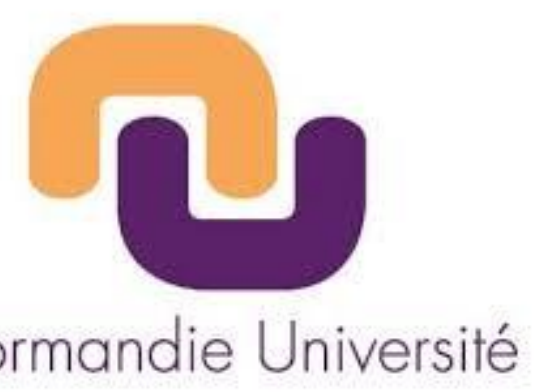

\title{
Enseignements à distance
}

Des arts savants, le génie d'un métier, l'instruction en valeurs : anthropologies d'une organisation éducative

\section{Olivier Marty}

Chercheur associé à l'université de Rouen (France) 
Sommaire

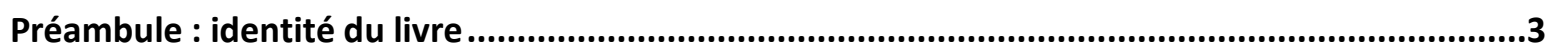

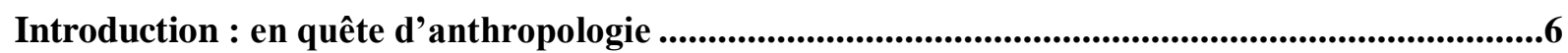

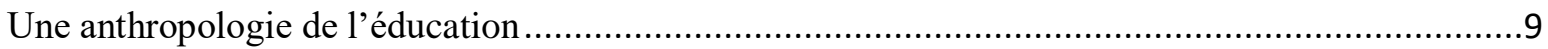

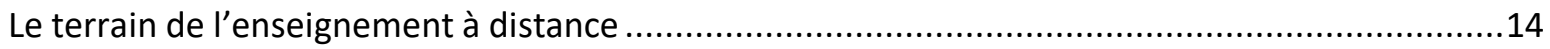

La méthode d'une présence prolongée en institution éducative ................................................17

Le statut des données : le récit d'expérience ...........................................................................19

Résultats de recherche dans les métiers de la formation ..........................................................21

Une institution industrielle de l'enseignement à distance ...................................................................41

L'entrée « management » dans l'enseignement : comptabilité, mercatique, gestion de projet ....51

La valeur comptable d'une formation en sciences de la gestion..................................................54

La carte commerciale : un outil de marketing de la formation ..................................................64

Le «projet G. » : le geste et son effet dans la conception de formation ..........................................70

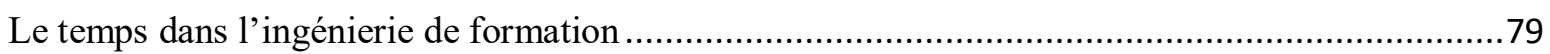

Les métiers de l'ingénierie de formation : variations sur les ingénus.......................................93

Le génie coopératif : contribution à l'ethnologie des opérations conjointes .................................96

Les arts et métiers d'une industrie éducative : des compétences aux outils ..................................115

L'engin des théâtres numériques et leur vocabulaire.................................................................126

Un exemple de conception en organisation éducative : la licence ouverte ....................................134

La sortie du terrain : les valeurs de l'apprentissage ..................................................................139

Le droit chemin : une méthode pour apprendre ...................................................................141

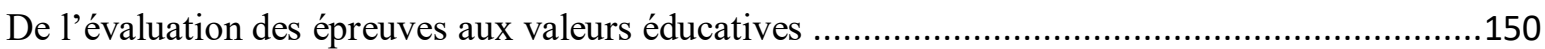

Retours sur la mesure de la valeur : que vaut un apprentissage ? ...........................................172

Conclusion : de l'anthropologie économique à l'anthropologie épistémique .................................177

Revue de littérature : préciser l'anthropologie de l'éducation par celle des savoirs .....................178

Au-delà de la notion de métier : les anthropologies mobilisées ................................................182

Une épistémologie équilibrée entre constructivisme et positivisme .............................................187

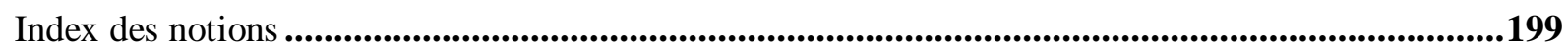

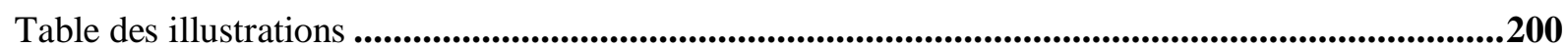

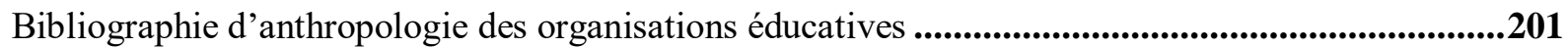

Ce livre s'est écrit en sept ans, entre 2012 et 2019. Le lecteur pressé pourra se concentrer utilement sur les titres que nous mettons en relief : le préambule puis les parties introductives de chacun des chapitres de premier niveau. C'est-à-dire le début de "l'introduction », " une institution industrielle dans l'enseignement", "l'entrée 'management' dans l'enseignement», "les métiers de l'ingénierie ", "la sortie du terrain » et la fin de la " conclusion ». L'appareil de lecture en bas de sommaire est également utile : index des notions, illustations, bibliographie non chapitrée. 
Ce livre fait état d'un terrain de trois années dans une institution d'enseignement à distance (2011-2014). Certains résultats ont été confimées par une de mes étudiantes qui est revenue sur le même terrain (2015) pour effectuer un terrain et confronter les résultats aux réalités, toujours mouvantes. L'équipe des enseignants étudiés s'est montrée compréhensive et nous les en remercions.

Les chapitres centraux, ouverts par une description générale de l'organisation éducative ${ }^{l}$ étudiée, montrent le rapport au terrain et la construction du point de vue du chercheur, déterminé par des préparations et un parcours antérieur : le regard n'est pas vierge et j'utilise mes préconceptions. Cet ouvrage s'intéresse donc aux liens entre formation et emploi. Le document décrit la situation d'emploi et comment sont mobilisées les formations dans l'action - et comment elles s'y déforment et s'y affinent dans le temps long. C'est l'usage des connaissances ou encore l'opérationnalisation des savoirs qui est à l'examen.

Après un diplôme d'études approfondies au département de sciences sociales de l'École normale supérieure, avec un mémoire d'ethnographie du travail innovant au Collège de France, je me suis inscrit dans une grande école de commerce dont j'ai décrit les exercices d'apprentissages dans un projet de recherche ${ }^{2}$. J'acquérais alors une éthique de l'action rénovée par une vague venue d'outre-Atlantique: mercatique, finance, comptabilité, ressources humaines, gestion de projet, etc. - et ses anglicismes. Le terrain d'observation présenté dans cet ouvrage est un site historique de l'enseignement à distance français dédié à l'enseignement post-bac. La recherche est l'occasion de montrer la logique gestionnaire à la manœuvre pendant trois années, dans ce qu'il est convenu d'appeler la nouvelle gestion publique $^{3}$ des cadres intermédiaires d'une industrie éducative (les «responsables de formation »). L'objet du travail scientifique est de porter un regard, formé par une école professionnelle, sur une institution se transformant elle-même vers ces formes. Ce ne sont pas les conceptualisations ordinaires des acteurs qui sont étudiées, mais les concepts savants,

\footnotetext{
${ }^{1}$ Nous préférons le terme d'« organisation» (du grec ergein, le travail) à celui, plus commun, d'institution (instituante car instituée) ou même d'agence, qui porte l'idée d'une action intentionnelle. Le terme, en vogue outre-Atlantique avec l'organizational analysis portée par le département d'éducation de l'université de Stanford, est perçu en France par le prisme des métiers. Ainsi il est possible de lire cette anthropologie d'une organisation éducative comme une anthropologie des métiers de l'enseignement (à distance).

${ }^{2}$ Document de travail publié en ligne : https://halshs.archives-ouvertes.fr/halshs-01233788.

${ }^{3}$ Quoique ce terme de New Public Management, avec ses controverses éclairant l'actualité du terrain, soit en perte de légitimité, l'évolution des savoirs laissant place à d'autres formes déconomicismes appliqués à l'action.
} 
appris académiquement lors d'une formation, que j'observe à l'œuvre et opérationnalisés dans l'action. Ainsi le premier chapitre central montre comment les sciences de gestion sont utilisées par les acteurs de terrain dans leurs opérations concrètes. En tant que chercheur j'utilise pleinement les cadres de pensées issus d'une formation préalable pour regarder une réalité qui s'y plie, à la fois dans ses pratiques et ses discours idéologiques (mercatique et finance de la formation, gestion de projet). Ce sont ainsi des arts savants qui sont décrit : l'habileté ou la compétence des professionnels est façonnée par la formation.

L'entrée gestionnaire, qui instrumentalise les sciences de gestion pour la description d'une institution d'éducation, débouche ensuite sur l'observation-apprenante des activités d'ingénierie de formation à distance - auxquelles je n'avais pas été auparavant formé et pour lesquelles je portais donc un regard plus neutre. Mes idéations d'ethnographe autour de l'ingénierie sont plus originales car moins instituées : c'est la déclinaison de l'ethnie des ingénus et de leur génie propre - ou l'art de manier les engins éducatifs et les métiers de la pédagogie numérique. Il s'agit du génie d'un métier ou des arts de manier un outil. Les ingénieurs ont de de l'expérience dans l'utilisation de leur instrument de travail, encore défini comme leur engin-propre. Ce deuxième chapitre central montre des points de vue sur l'ingénierie de formation à partir d'une logique philosophique candide - n'ayant pas été formé à l'ingénierie de formation et l'observant en m'acculturant à ces réalités étrangères, et par làmême étranges, avec des étonnements généraux (génie, engins, exemple de projet). Les conditions sociales de l'enquête habituellement retenues (je suis fils d'ingénieur) ne sont pas développées car jugées peu influentes sur ce travail de recherche (quoiqu'elles sont suscité une passion à l'égard de l'objet).

Le dernier des trois chapitres centraux prolonge l'entrée gestionnaire sur les métiers de l'ingénierie de formation par une sortie sur les valeurs de l'apprentissage à distance. Ayant été en partie formé par l'enseignement à distance (obtention de licences universitaires), je fonde l'évaluation de l'activité professionnelle sur les valeurs issues de mes expériences d'apprenant. C'est un regard de l'apprenant sur le professionnel, mais aussi de la génération du papier sur celle du numérique en matière d'enseignement à distance. Ce troisième chapitre central montre les valeurs de l'apprendre dans l'enseignement à distance et, là, j'ai recours à ma propre formation d'étudiant à distance pour comparer les pratiques professionnelles que j'observe avec les expériences que j'ai vécues en tant que destinataire des productions (théorie de l'activité, types d'évaluation). C'est ainsi l'instruction, élévation intérieure conséquente à toute éducation, qui est évaluée et donc mise en valeur par ses qualités et quantités. 
L'introduction et la conclusion de l'ouvrage sont issues d'une habilitation à diriger les recherches en sciences de l'éducation, intitulée «Anthropologies des organisations éducatives ». Ces deux parties présentent les résultats d'une collaboration avec le Centre de Recherche sur la Formation (devenu Formation et Apprentissages Professionnels en 2019) du Conservatoire national des arts et métiers, pour fonder le récit des expériences de terrain. La partie introductive apporte des éléments d'insertion dans le champ des sciences de l'éducation française, ce sont les apports sur différents objets : communication, conception, organisation, identité... La conclusion est un prolongement épistémologique de ma thèse de doctorat en philosophie : équilibre entre constructivisme et positivisme, ouverture vers une anthropologie épistémique pour comprendre l'institution de diffusion en masse des savoirs.

Nous avons hésité à intituler ce livre «Les ingénus ». Ceci s'explique par une double référence au chapitre le plus central sur l'ingénierie de formation - et cela peut paraître paradoxal lorsque l'on évoque l'enseignement. D'abord parce qu'il me différencie, en tant qu'enquêteur, doublement asservi par l'institution étudiée me salariant et par le laboratoire de recherche ou je suis affilié, par rapport à des ingénieurs ingénus que j'étudie ouvertement et qui sont plus libres car ne dépendant que de l'organisation les rémunérant. Ce titre fait aussi référence au même chapitre où la production du regard ethnographique est ingénu car moins formé ou préparé à observer des réalités selon des cadres préconçus. Ce chapitre se distingue en cela de ceux, instrumentant les sciences de gestion ou une expérience d'étudiant dans l'enseignement à distance, qui font l'identité du livre : présenter les préconceptions pour expliquer des observations, jamais neutres. Par ce titre où je voulais mettre à distance « les ingénus », je voulais me détacher donc à la fois des observés sur le terrain et des observateurs traditionnels : je me présentais, non comme un ingénu, mais comme un chercheur assumant ce qui génère ses observations scientifiques - un sceptique.

Le titre choisi « Enseignements à distance » éclaire à la fois l'organisation étudiée (qui effectue l'opération d'enseigner à distance) mais aussi ceux qui y travaillent (les enseignants) et ce qu'ils font (des enseignements à distance). C'est aussi la signature même du livre qui constitue un ensemble d'enseignements à distance sur son objet, ainsi mis en relief. 


\section{Introduction : en quête d'anthropologie}

La numérisation de l'enseignement supérieur est une transformation majeure de ce tournant de siècle, avec la transition de la formation assistée par ordinateur (les didacticiels) vers des environnements informatiques pour l'apprentissage humain (EIAH) complets, connectés à Internet et interactifs. Ainsi la pédagogie universitaire devient numérique et laisse une part aux Internet Studies pour comprendre les dynamiques de groupe en ligne, l'apprentissage social dans les réseaux et les interactions avec les programmes informatiques.

Dans ce cadre-là, les institutions d'enseignement à distance figurent comme des avantgardistes qui peuvent valoriser leurs expériences pédagogiques et leur didactique propre, vieilles de plus d'un demi-siècle, tout en expérimentant les nouveaux outils de formation. Les universités, qui sont à présent toutes connectées, peuvent bénéficier de ces apports et augmenter leurs pratiques.

Nous avons mené une étude ethnographique de trois années de présences dans une institution française d'enseignement à distance, opératrice de l'état, entre 2011 et 2014. Ce alors que l'organisation migrait ses plateformes numériques depuis un «campus électronique » remontant aux années 1990 vers un dispositif Moodle et Blackboard plus moderne. L'institution étudiée lors de ce terrain, et dont nous nous efforcerons de garder l'anonymat, gardait la trace sédimentée des précédentes technologies : d'imposantes rotatives fonctionnaient encore pour l'impression de cours stockés dans un entrepôt et les envois de copies faisaient l'objet d'accords pour des sacoches postales spécifiques ${ }^{4}$.

Notre étude a donné lieu à plusieurs publications de sciences de l'éducation que nous avons synthétisées autour de ce même thème de l'enseignement à distance. Nous voulons aujourd'hui présenter une partie de ce travail exposant nos principaux résultats, mais aussi des documents inédits qui permettent de compléter notre approche.

Nous verrons ainsi l'intention anthropologique en sciences de l'éducation qui anime notre propos : la comparaison entre des terrains de plusieurs métiers du numérique et de plusieurs aires géographiques permet en effet d'obtenir des résultats généraux concernant l'homme dans ses activités d'apprentissage. Nous situons ainsi notre anthropologie entre l'anthropologie des institutions éducatives américaine et la nouvelle anthropologie des

\footnotetext{
${ }^{4}$ Toutefois, l'objet de ce livre ne sera pas d'accumuler des descriptions du terrain ethnographique mais d'en
} extraire des abstractions anthropologiques 
savoirs. C'est une anthropologie des métiers tout autant épistémique qu'économique - ce sur quoi nous reviendrons en conclusion.

Après une présentation du terrain et de notre position d'enquêteur employé par l'institution, nous verrons le statut des données produites avant de lister plusieurs résultats par objets de recherche. Nos travaux nous ont permis de développer une méthode d'étude des déontologies (valeurs en actes, discours sur les normes et règles en vigueur) en organisations (entreprise, institution, administration) que nous avons appliquée au champ des métiers de la formation à distance des adultes. Nous avons ainsi montré, dans une série de publications, les conflits de valeurs entre catégories d'employés de l'enseignement à distance et leur harmonisation par un discours académique. Nous avons alors travaillé le champ des métiers de la formation à distance des adultes par des objets regroupés en trois axes, au Cnam et à l'université de Lille : le sens (identités et valeurs la formation comme la pédagogie et la didactique), les pratiques (la professionnalisation, les activités et notamment de conception) et les outils (médiatiques, organisationnels ou linguistiques comme la communication et le vocable de l'apprentissage) - nous présenterons ces objets dans l'ordre chronologique dans lequel ils ont été travaillés pour mieux saisir la construction naturelle de notre pensée.

Nous proposons ainsi une définition ternaire du métier, s'appuyant sur nos trois axes : c'est d'abord une expérience (« avoir du métier») qui donne sens à une communauté de pratiques («les gens du métier») se servant d'un outil (tel le «métier à tisser»), ici organisationnel et linguistique du fait de l'objet formation à distance. Les autres termes de notre titre feront l'objet d'un approfondissement dans la présentation de nos objets : la formation et l'idée de forme avec le suffixe -tion qui indique le mouvement vers les résultats d'apprentissages ; la distance avec l'inscription des signes dans un média ou support tangible.

Notre atout ethno-méthodologique est d'aborder ces objets sur le terrain avec plusieurs lunettes : celles des sciences sociales du fait éducatif, augmentées par le discours de justification philosophique, mais aussi celles des sciences de gestion et politiques pour l'accès $\mathrm{au}$ terrain et la proposition d'objets nouveaux (liberté, égalité). La synthèse à visée anthropologique s'appuie sur des abstractions issues de comparaisons: entre des terrains professionnels différents (nouvelle économie, fabrication de pirogues, profession artistique, enseignement à distance) et des analyses essentiellement linguistiques pour les aires 
culturelles complémentaires (comme pour l'arabe littéraire ${ }^{5}$ ) mais aussi des différentes cultures disciplinaires impliquées (par exemple la discussion du vocable juridique et des termes mathématiques).

La partie principale de l'ouvrage découle de notre positionnement d'enquêteur. Nous verrons en effet, après une brève contribution institutionnaliste présentant l'organisation étudiée, classique en anthropologie de l'éducation, que nous avons tenté d'instrumenter les sciences de la gestion - qui donnaient accès au terrain - en réfléchissant anthropologiquement à ses cadres d'analyse : la finance et le calcul de la valeur d'une formation, la mercatique et l'image de marque d'une institution d'enseignement voulant vendre ses produits ou encore les techniques de projet avec la conception finalisée du temps qu'elles impliquent.

Ceci nous amènera alors à préciser notre approche en termes d'ingénierie de formation à distance. La description détaillée de projets d'ingénierie de formation au sein de l'institution sera l'occasion de réfléchir à leurs engins (les théâtres numériques ou plateformes d'enseignement) comme à leur génie propre (les arts relationnels et pédagogiques de ces métiers de la formation) qui font ce métier. Nous définirons à cet endroit ce que nous avons nommé l' «ethnie des ingénus » lors de nos hésitations d'intitulé, en expliquant les avantages polysémiques et laudatifs de ce terme.

Enfin nous sortirons progressivement de notre terrain dans l'enseignement à distance en nous intéressant à la question des apprentissages, de ses valeurs et de son évaluation. Notre terrain concernant d'abord les cadres intermédiaires d'une institution d'enseignement à distance sera ainsi l'occasion d'être confronté à des étudiants à distance (dont nous avons été) et de mieux comprendre leurs activités (la méthode du droit chemin dans l'apprentissage), les valeurs qui fondent les nouvelles épreuves pour tester les compétences professionnelles et enfin l'évaluation quantitative (le «combien» pour classer la masse par ordre de performance) et qualitative (le « qui » pour apprécier l'élite individuellement).

La conclusion de l'ouvrage sera réservée à une ouverture sur l'évolution de l'anthropologie économique vers l'anthropologie épistémique. Ce par une analyse de la

\footnotetext{
${ }^{5}$ Nous pouvons ainsi situer linguistiquement notre propos par des comparaisons culturelles qui légitiment la posture anthropologique : à la racine arabe DRS (moudaris, medersa, darsoun ; le professeur, l'école, la leçon), il peut être ajouté l'héritage humaniste gréco-latin (intention pédagogique, decorum, méthode curriculaire) ; mais aussi l'hindi dekhna, "montrer », voire dekhana, "faire montrer ", qui seraient à l'origine, selon I'hypothèse d'un bien commun indo-européen, de la racine doceo et donc du signe éducatif. Dans l'enseignement à distance, la professionnalité professorale, ou parole devant audience, se heurte au paradoxe de l'écrit muet, avec des signes parfois revivifiés par des dispositifs tels que la classe virtuelle ou les enregistrements audiovisuels.
} 
littérature scientifique et par des remarques épistémologiques sur le constructivisme, le positivisme et la praxéologie.

\section{Une anthropologie de l'éducation}

La perspective anthropologique en sciences de l'éducation est, loin d'être nouvelle, largement reconnue en France et à l'étranger ${ }^{6}$. Au-delà des divers manuels existant sur le sujet, sur lesquels nous aurons à revenir du fait des titres changeants, appelant des commentaires quant à l'objet retenu par la communauté anthropologique, il est à noter que l'actuelle liste des revues de la section en sciences de l'éducation française, reconnue par le Haut comité pour l'évaluation de la recherche et de l'enseignement supérieur en 2016, compte deux titres mentionnant directement les notions d'anthropologie (Anthropology and Education Quaterly) ou d'ethnographie (Ethnography and Education) et plusieurs autres avec les notions proches d'études qualitatives en science sociales, fondées sur des terrains d'observation participante (International Journal of Qualitative Studies in Education ou encore Qualitative Inquiry). Ce à quoi il faut ajouter que bon nombre de revues accueillent ce type de travaux, comme Journal of Distance Education ou International Review of Research in Distance Learning qui accueillent aussi cette perspective anthropologique pour étudier l'enseignement à distance.

Nous voulons à présent revenir sur cette perspective anthropologique en montrant ses usages modernes dans l'étude des mondes contemporains.

L'histoire classique de l'anthropologie la fait remonter au $19^{\text {ème }}$ siècle colonial et à ses explorateurs rapportant des nouveaux mondes des récits extraordinaires sur des us et coutumes différents qu'il restait à penser et à étudier en les comparants avec les nôtres. Cette histoire liée à l'époque coloniale est certes remise en cause à l'ère postcoloniale pour chercher des racines plus anciennes, le plus souvent, toutefois, en s'accordant sur le rôle prépondérant

\footnotetext{
${ }^{6}$ Une histoire, nécessairement incomplète, de l'anthropologie de l'éducation en France pourrait débuter avec les travaux d'ethnométhodologie d'Alain Coulon à l'université de Paris 8 Vincennes à Saint Denis dans les années 1990, puis à ceux d'anthropologie de l'éducation de Marie Louise Martinez à l'université de Rouen une décénnie après, avec les coordinations de travaux parallèles de Danvers. On note qu'en 2019 Brigitte Albero, présidente de la section sciences de l'éducation du conseil national des universités français, est une anthropologue de l'éducation. Nous apportons à ces différents travaux, que l'on pourra retrouver en bibliographie, une spécificité institutionnelle : notre anthropologie des organisations éducatives retrouve les notions de travail, d'activité et d'endroit de production propres à la démarche ethnographique, fondement de l'anthropologie.
} 
des récits d'explorateurs - ceux-ci pouvant être de la période européenne antique du temps d'Hérodote ou même issus symétriquement d'autres aires culturelles comme lorsque les lettrés musulmans, alors plus avancés, découvraient l'Europe du Moyen-âge au travers des dires et écrits des émissaires de leur propre civilisation.

C'est ainsi l'expérience de l'altérité radicale, sur le terrain, quelle qu'en soit sa date historique et les civilisations concernées, qui est au fondement de la pensée anthropologique. Lévi Strauss distinguera ainsi, lui aussi classiquement dans les années 1960, l'ethnographe qui va explorer et décrire une ethnie exotique en particulier; de l'ethnologue spécialiste de plusieurs ethnies d'une aire géographique (comme l'Amérique Latine par exemple) qu'il a pu expérimenter ou lire au travers d'études de collègues; et enfin de l'anthropologue produisant des généralités sur le genre humain au travers de comparaisons ethnologiques - les anglosaxons usant de l'image du armchair antrhopologist enfermé dans son cabinet de lecture et qui « hait les voyages et les explorateurs », leur préférant les récits écrits.

Cette conception qui a pu prévaloir jusque dans les années 1980 a cédé sa place à l'anthropologie des mondes contemporains, portée notamment par Marc Augé à l'École des hautes études en sciences sociales, où il s'agit d'user de la méthode d'enquête ethnographique pour étudier les recoins méconnus de nos propres sociétés : artistes, parlementaires, managers.... Ainsi l'anthropologie se rapprochait-elle d'une sociologie qualitative elle aussi en pleine essor, liée à l'interactionnisme symbolique de l'école de Chicago, l'observationparticipante et, plus largement, les ethno-méthodes prônées par Goffman, Garfinkel et Becker.

La principale difficulté, de nature épistémologique, a été de conserver l'expérience de l'altérité ou le sentiment d'étrangeté au fondement du travail ethnographique. Ainsi le développement de l'ethnographie des organisations, qui a ses manuels anglo-saxons et ses directeurs de recherche (Michel Villette, Nicolas Flamant et, dans une moindre mesure, Patrick Fridenson) s'est-elle confrontée à des questions identitaires pour les chercheurs. Notre ethnographie des organisations éducatives, à visée anthropologique par comparaison de différents terrains professionnels, a approfondi, cette question de l'identité lors des terrains longs. Des notions anglo-saxonnes telles que les «halfies» sont ainsi d'utilité dans les épistémologies contemporaines sur ces questions («halfies» désigne les fils d'indigènes travaillant comme ethno/anthropologues, et nous le sommes relativement dans le sens où nous sommes issus d'une famille d'enseignants - nous avons proposé le terme de « hybrides » pour désigner ces chercheurs-là). 
On voit ainsi comment la pensée anthropologique de l'éducation, du moins celle du $20^{\text {ème }}$ siècle, telle qu'elle est présentée dans le manuel Visionary Observers. Anthropological Inquiry and Education ${ }^{7}$, a évolué d'anthropologues généralistes issus de terrains exotiques (Franz Boas, Ruth Benedict, Margaret Mead, Gene Weltfish, Hortense Powdermaker...) et présentant quelques résultats sur le fait éducatif dans différentes cultures; vers des anthropologues des mondes contemporains spécialisés dans le fait éducatif, auquel nous nous rattachons et pour lequel nous défendons la position que la généralisation est possible en comparant les métiers (et non pas des aires de géographie culturelle).

La qualification d' «anthropologique » de nos travaux tient ainsi non pas tant à un premier travail de recherche mené dans l'ethnie Djuka sur les rives du Maroni au nord de la Guyane française - terrain relativement classique dans sa forme exotique quoique qu'il ait débouché sur une réflexion épistémique d'architecture des savoirs - mais plutôt à cette particularité des différents métiers comparables au travers de mondes contemporains par des ethnographies en organisation : les entreprises évanescentes des Start Up et les métiers de l'innovation, ou encore l'ingénierie de formation à distance que nous présentons ici, voire la formation des cadres religieux dans une institution monothéiste. Ainsi ces différents terrains successifs permettent de s'abstraire de mondes contemporains en retirant de leur fréquentation des schémas explicatifs transposables.

Considérer les enseignants comme la culture d'un métier propre qui appelle à être examinée en tant qu'ethnie (en reprenant les concepts anthropologiques de rites, d'initiation, d'identité, de dimension spatiale cachée ou encore de temporalité propre, etc.) est donc une perspective qui peut poser des questions déontologiques et de réception des travaux par les mêmes enseignants ${ }^{8}$, pouvant être tentés de résister à l'objectivation en arguant de la confidentialité ou de l'intégrité du chercheur; mais cela s'inscrit dans une continuité de l'histoire des sciences qui fait de la position scientifique une attitude relativement normale et ancrée dans les mœurs de la recherche.

La question de la présence sur le terrain appelle celle des traces et notes de recherche qui différencient le chercheur réflexif du simple acteur. Au moment de l'étude, car il est en phase de recueil de données et de réflexions exploratoires. Pendant et après l'étude, pour

\footnotetext{
${ }^{7}$ Collectif, 2006, Visionary Observers. Anthropological Inquiry and Education, University of Nebraska Press. Lincoln and London. USA.

${ }^{8}$ Ainsi, dans le cas particulier de l'ethnologie de l'éducation, l'ouvrage de méthode qui pose ces questions de controverse et d'intégrité : Hammersley M, Traianou A., 2012, Ethics in qualitative research. Controversies and contexts, Sage, Londres.
} 
valoriser ce qu'il a trouvé auprès de la communauté scientifique sous différentes formes. Nous accordons ainsi une place importante aux moyens de la recherche : la méthode du carnet de terrain (complété par le film ethnographique : pour tirer parti de la révolution des caméras téléphoniques et de la diffusion plateforme vidéo pour la recherche de terrain, tout en préservant l'anonymat scientifique) et l'écriture réflexive. Les modalités entre le chercheur pur et le praticien avec une écriture réflexive fait l'objet de nuances multiples selon les situations d'études et les intentions de chacun.

Comme nous l'avons esquissé dans la conclusion à notre intervention orale à la Biennale de l'éducation $2015^{9}$, un principe perdure : une réflexion structurante pour définir les conditions pour passer de l'ethnographie à l'anthropologie. Ceci en accumulant des expériences réflexives dans des terrains éducatifs variés, dans des aires complémentaires afin d'en abstraire des modèles théoriques à la généralité croissante. Les terrains possibles à venir sont nombreux : l'enseignement supérieur et sa politique dans les lieux de gouvernance, l'enseignement à distance et ses plateformes numériques, la formation d'adultes dans le privé, les communautés éducatives en lien avec les établissements primaires et secondaires, les déontologies des disciplines avec leurs controverses en organisation éducative, etc.

On voit dans la succession de nos terrains ethnographiques passés la construction d'une démarche anthropologique visant à abstraire des principes généraux de leur multiplicité (on se reportera ainsi au triangle de l'analyse des distances, infra.). Il s'agit de terrains en milieux professionnels (édition numérique de la nouvelle économie et mode de travail traditionnel dans la jungle guyanaise, puis organisations éducatives : grande école, centre d'enseignement, organisme de formation professionnelle) qui se spécialisent dans différents postures éducatives (apprenant, enseignant, ingénieur) complémentaires. Le terrain de la thèse, concernant les professions taurines, apporte la dimension de l'étude des déontologies et est complété par un travail bibliographique.

\footnotetext{
${ }^{9}$ Marty O., 2015, Un modèle d'ingénierie coopérative. Contribution à l'ethnologie des opérations conjointes : l'algèbre du moment. Biennale de l'éducation, de la formation et des pratiques professionnelles 2015 : "Coopérer ? ", Jun 2015, Paris, France. $\leq$ halshs-01181335>.
} 


\begin{tabular}{|c|c|c|}
\hline Terrain & Année & Résultats \\
\hline $\begin{array}{l}\text { Start up, organisations } \\
\text { innovantes }\end{array}$ & $2000-2004$ & $\begin{array}{l}\text { Le sociogramme interagit avec l'organigramme, la parole est distribuée } \\
\text { en associant des mots et des interlocuteurs, les jeux d'alliances visibles } \\
\text { dans les regards sont déterminés par les relations structurelles. Trois } \\
\text { livres publiés, un article de recherche }\end{array}$ \\
\hline $\begin{array}{l}\text { Construction } \\
\text { pirogues en Guyanne }\end{array}$ & 2001 & $\begin{array}{l}\text { Le physique (voire juridique) détermine le social qui cadre le psychique, } \\
\text { un article de recherche }\end{array}$ \\
\hline Grande école & 2001-2005 & $\begin{array}{l}\text { L'action collective s'inscrit dans le temps avec ses dimensions de } \\
\text { mémoire collective, de cadre matériel et de stratégie futures. Un } \\
\text { document de travail publié dans HalsSHS }\end{array}$ \\
\hline $\begin{array}{l}\text { Théâtre et jeux de rôles } \\
\text { pédagogiques }\end{array}$ & 2003-2013 & $\begin{array}{l}\text { Les jeux de rôles sont des outils pédagogiques qui permettent de faire } \\
\text { vivre les langues et de s'identifier à des rôles professionnels, le théâtre } \\
\text { sert la formation notamment par l'apprentissage de la vie sociale. Un } \\
\text { dossier coordonnée pour une revue scientifique }\end{array}$ \\
\hline $\begin{array}{l}\text { Déontologie de la } \\
\text { profession de torero }\end{array}$ & $\begin{array}{l}\text { Acculturation } \\
\text { précoce, } \\
\text { thèse en } \\
2008-2011\end{array}$ & $\begin{array}{l}\text { Les valeurs déontologiques d'une profession (courage, honneur, loyauté) } \\
\text { controversée sont légitimées par la littérature. Thèse de doctorat publiée } \\
\text { sur www.theses.fr }\end{array}$ \\
\hline $\begin{array}{l}\text { Centre national } \\
\text { d'enseignement à } \\
\text { distance }\end{array}$ & $2011-2014$ & $\begin{array}{l}\text { Les métiers en présence dans l'institution font appel à des arts ou } \\
\text { compétences novatrices dans l'enseignement. Le calcul de la valeur } \\
\text { d'une formation selon le New Public Management se heurte aux valeurs } \\
\text { traditionnelles des acteurs, c'est un conflit déontologique en } \\
\text { organisation. Quatre articles de recherche bilingues publiés. }\end{array}$ \\
\hline Serious game & $\begin{array}{l}\text { (terrain de } \\
\text { seconde } \\
\text { main) }\end{array}$ & $\begin{array}{l}\text { Un didacticiel est un média qui force l'expérience d'apprentissage et } \\
\text { présente des biais épistémiques liés à son historique de constitution. Un } \\
\text { chapitre dans un ouvrage scientifique collectif }\end{array}$ \\
\hline
\end{tabular}


Entre 2011 et 2014 nous fûmes donc dans un double cadre institutionnel : le terrain du « Centre d'éducation à distance » $(\mathrm{Ced})^{10}$ nous occupait les jours ouvrés et le laboratoire du Conservatoire national des arts et métiers (Cnam) servait d'appui réflexif au miroir de ses axes d'analyse durant les heures de repos et de congés.

De ce terrain important dans notre parcours de chercheur, centré sur l'enseignement à distance supérieur du site de Vanves mais rayonnant par curiosité sur les autres sites du Ced et quelques comparaisons internationales, nous avons retiré tout d'abord deux documents de travail et trois articles de recherche complémentaires.

- Michael Grahame Moore, Olivier Marty.. 2015 «La théorie de la distance transactionnelle ». Trad. de M. G. Moore, The Theory of Transactional Distance. Handbook of Distance Education, 2007. $\leq$ halshs-00777034>

Le premier réflexe fut, lors de la période d'acquisition de la littérature sur l'enseignement à distance, de traduire un chapitre reconnu important d'un manuel récent sur le sujet - celui de M.G. Moore sur la distance transactionnelle. Avec l'autorisation de ce dernier et de l'éditeur de publier ma traduction sur HALSHS en document de travail.

Cette traduction allait influencer nos réflexions ultérieures sur l'enseignement à distance, notamment en reprenant son axiomatique orthogonale « dialogue » versus « structure » par les termes de «pédagogie » et de «méthode», qui allaient servir dans plusieurs schémas pour penser l'enseignement à distance et que nous présenterons ci-après.

Après ce premier document de travail, nous nous lançâmes dans la publication successive de trois articles de recherche.

- Olivier Marty. 2014. «Monetizing French Distance Education. A Field Enquiry on Higher Education Value(s) ». The International Review of Research in Open and Distance Learning, 2014, vol. 15 (n 2), <10.19173/irrodl.v15i2.1677>. 〈halshs-00994600>

Ce premier article est une inscription de l'enseignement à distance français dans l'histoire du système d'enseignement supérieur européen, précisé par une observation fine du quotidien et des valeurs distinctes des différents employés actuels (selon la méthode de l'analyse

\footnotetext{
10 Nous nous efforçons de présever l'anonymat de cette institution, notamment par l'invention de cet acronyme.
} 
organisationnelle des pratiques déontologiques en éducation : nous centrant sur les valeurs portées par chaque catégories d'employés et leurs éventuels conflits), notamment autour de la question du calcul de la valeur d'une formation dans le cadre du New Public Management.

Le positionnement ethnographique était relativement simple puisque, nouvellement arrivé dans les fonctions, nous étions encore suffisamment «étranger» aux individus observés pour pouvoir porter un regard neutre et neuf sur l'institution. Un regard suffisamment large et général pour intéresser les acteurs extérieurs au monde de l'enseignement à distance et les chercheurs du champ dans d'autres pays.

Le choix d'une revue de langue anglaise, canadienne, permettait de donner du sens à la description à grandes lignes du système éducatif français pour permettre les comparaisons internationales.

- Olivier Marty. 2015. «Professionnalisations à l'ingénierie de la formation à distance : comparer formation par le travail et formation au travail. » Travail et Apprentissages, p. 103115. 〈halshs-01239986>

Ce second article sur le thème de la professionnalisation correspond à une période où nous pouvions dresser un bilan introspectif sur nos apprentissages professionnels et l'objectiver par la mission de formation de nouvelles recrues que nous avions dans l'emploi.

Y sont comparées notamment la professionnalisation par le travail, sur poste et dans l'institution avec la professionnalisation que nous menions par des actions de formation de type conférencier invité en Sorbonne pour le doctorat professionnel ou formateur à la classe virtuelle de partenaires universitaires.

Le choix de la revue Travail et Apprentissages était lié à la lecture de la didactique professionnelle de Pierre Pastré et la volonté d'intégrer ces réflexions à nos propres travaux. Nous aurons l'occasion de développer cette description dans l'objet de recherche «professionnalisations »

- Olivier Marty. 2016. «Penser l'enseignement à distance. Valeurs historiques, économiques et esthétiques d'un nouvel élitisme» International Journal of E-learning and Distance Education, Athabasca University, 2016, vol. 32 ( $\mathrm{n}^{\circ}$ 1), <http://www.ijede.ca/index.php/jde>. $\leq$ halshs-01348565>

Enfin le troisième article scientifique marque un aboutissement des recherches sur deux dimensions. D'une part, car il est publié dans le Journal of Distance Education, revue 
emblématique du champ d'étude marquant une certaine reconnaissance scientifique internationale.

D'autre part, parce qu'il marque une évolution temporaire de l'ethnographe vers le philosophe. En effet, reprenant la genèse de notre formation pré-doctorale en sciences sociales puis doctorale en philosophie, nous effectuions le même mouvement dans la recherche sur l'enseignement à distance en commençant par publier en ethnographe décrivant les valeurs du champ étudié, puis en reprenant et portant ces mêmes valeurs comme un acteur du champ. Le processus d'indigénisation allait jusqu'à son terme puisque l'article semblait émaner d'un acteur du terrain, portant des valeurs synthétiques (économiques, historiques, esthétiques) valables pour tous les métiers de l'enseignement à distance et voulant les promouvoir.

L'analyse des métiers de la formation à distance qui est présentée dans l'article et qui sert le propos de la synthèse est ainsi en partie doublé d'un discours endogène clamant la valeur d'un élitisme par l'enseignement à distance, quittant le descriptif pour oser le prescriptif selon les canons prospectifs du milieu observé - et l'influence de Gaston Berger.

Cette évolution, peut être naturelle lorsque l'on est dans un terrain long en milieu éducatif, mérite d'être étudiée en soi dans l'analyse du travail du chercheur qui, aux prises avec ses identités multiples, voit son discours scientifique évoluer avec son intégration dans le terrain observé.

Ce mouvement se parachève avec le cinquième document présenté :

- Olivier Marty. 2016 « Licence ouverte. Utilisation des Cloms pour obtenir le grade de licence. » [Rapport Technique] Direction générale de l'enseignement supérieur et de l'insertion professionnelle. 〈halshs-01329191>

Ce document est un rapport qui a été adressé au ministère de l'enseignement supérieur et de la recherche en 2016 et qui a donné lieu à une audition à la direction générale de l'enseignement supérieur et de l'insertion professionnelle après une expertise directe par la directrice Simone Bonnafous. Il s'agit d'un rapport d'ingénierie présentant un nouveau curriculum possible avec ce qui est présenté comme l'enseignement à distance de nouvelle génération : les Moocs (ou Cloms en français).

Le propos du rapport est de quantifier et planifier la création d'un diplôme de licence obtenu par accrétion de Moocs. Il a donné lieu à un complément d'ingénierie par les agents du 
ministère proposant d'abord un diplôme d'établissement pour ensuite être soumis aux instances nationales pour devenir diplôme national.

Ici la recherche est finalisée et objectivée - pour reprendre les catégories descriptives et classificatoires de la stratégie nationale de recherche en vigueur en 2015, elle quitte le caractère descriptif pour entrer au service de la production. Le discours produit est celui d'un indigène et il intéresse essentiellement d'autres acteurs du même champ.

Le mouvement que nous avons esquissé dans la présentation chronologique de ces publications de terrain, de l'ethnographe vers le philosophe, du scientifique vers l'indigène technique, fera l'objet d'un article méthodologique que nous allons présenter.

\section{La méthode d'une présence prolongée en institution éducative}

Nous voulons donner une place particulière à une de nos publications d'article de recherche. Elle a en effet trait à la méthode, sujet que nous avons travaillé relativement précocement. En effet, dès notre mémoire sur les Start Up, nous avions inclus un appendice «Observer, lire et écrire » analysant notre propre travail de chercheur. Ceci s'est prolongé par un mémoire de fins d'études à l'Édhec portant sur l'ethnographie des organisations. Il s'agissait pour nous de décrire le champ, l’objet, les méthodes et les premiers résultats de ce que nous qualifions de discipline récente.

Lorsque nous sommes revenus à l'analyse des mondes professionnels, après la thèse, nous avons rédigé une synthèse de nos travaux autour de la thématique de la méthode.

- Olivier Marty. 2014. «A model of distance analysis. Epistemic field notes for education ethnographers ». Ethnography and Education, Taylor \& Francis (Routledge), 2014, vol. 10 (n 1), p. 17-27. $\leq 10.1080 / 17457823.2014 .922892>$. 〈halshs-01010283>

- Olivier Marty. 2013, «An epistemology of distance. Ethnography of training activities : engineering and management in higher education organizations ». International Conference. Ethnographies of higher education : researching and reflecting "at home", May 2013, Prague, Czech Republic. $\leq$ halshs-00833739>

Cet article a été préparé par une conférence à Prague où nous avons rencontré notre éditeur fondateur de la revue Ethnography and Education. Son attention a été retenue par notre présentation et il a proposé de la développer en article de recherche. 
Ainsi nous nous sommes efforcés de classer nos terrains et travaux ethnographiques selon un modèle des distances - modèle qui a été retravaillé pour Oxford Ethnography and Education Conference 2019 (OEEC 2019), avec le même éditeur. Ce modèle tridimensionnel croise les distances sociales au terrain et à la communauté scientifique à la distance psychique à soi (ordonnée en fonction des deux premières); qui varient toutes selon le moment de l'étude et les affinités structurelles du chercheur avec son terrain. Ainsi l'axe vertical du psychologique est ordonné par l'axe horizontal du sociologique en abscisse. La distance psychique à soi-même est fonction de la distance sociale (qualifiée de distance au terrain d'un côté et de distance à la communauté scientifique de l'autre) ; le tout constituant un modèle de méthode anthropologique utilisé en sciences de l'éducation. Nous pensons qu'un tel modèle n'est valable que dans le cadre d'un terrain long, où les effets psychiques du social se font sentir, et tout particulièrement dans le champ éducatif rapprochant le chercheur scientifique de l'éducateur indigène.
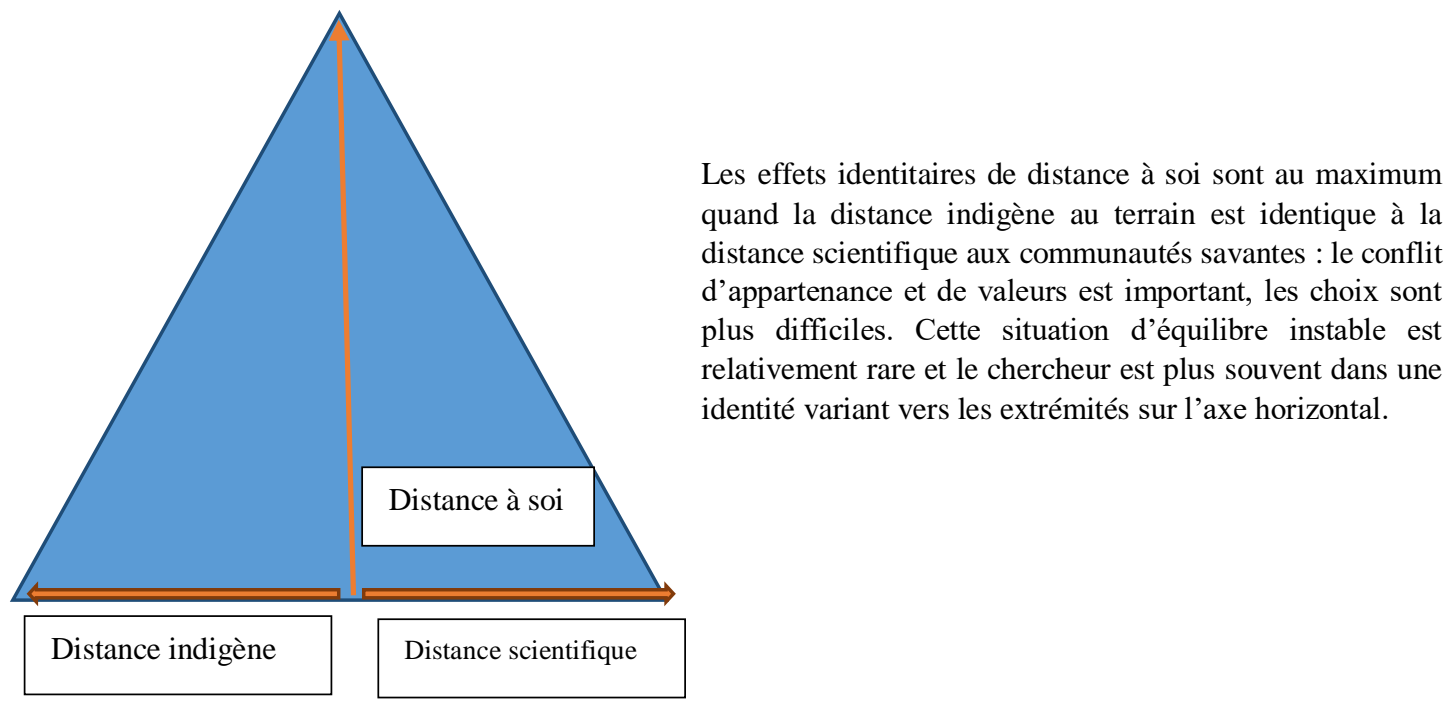

Le modèle d'analyse des distances

Ainsi nos données ethnographiques dans le terrain tauromachique ont montré une faible distance au terrain (notre enfance et expériences de jeunesses nous plaçant proche des 
indigènes) alors que les études sur les organisations innovantes (Start Up) et traditionnelles (construction de pirogues Djukas) nous plaçaient plus proches des communautés scientifiques que des populations observées.

Le cas particulier du terrain ethnographique de longue durée en éducation montrait des évolutions des distances (sur l'axe horizontal en bas de graphique) selon le moment de l'étude et le degré d'engagement dans les organisations étudiées, avec des moments d'hésitation créant des effets de distance à soi et de choix difficile lorsque l'on se sentait également chercheur scientifique et cadre éducatif sur le terrain.

Enfin remarquons, dans ces éléments méthodologiques, la place des mathématiques (qui est notre formation dominante au baccalauréat et que nous avons prolongée en autoformation) : le modèle fonctionnel des distances présenté ci-dessus et relevant de l'analyse a été complété par la notion d'algèbre éducatif (qui a été discutée en France et qui, je le défends, dépasse, par sa portée, le simple exercice de style, quoique cela pourrait aussi être un oxymore). La référence à la théorie des ensembles (et donc à l'étymon arabe g-b-r) en éducation permet de renouveler le vocabulaire commun de l'analyse par celui de l'algèbre en insistant sur ce qui rassemble plusieurs apprenants dans une forme commune. Nous présenterons les résultats liés à cet axe méthodique avec la notion de théâtre et d'approcheprogramme (renvoyant au processus de Bologne).

\section{Le statut des données : le récit d'expérience}

La méthode du terrain long en institution éducative, où le tiret liant deux fonctions souvent regroupées à l'université, l'enseignement et la recherche, est là distendu et défait entre deux institutions (cadrer les enseignements au Cned et rechercher au Cnam), implique des questions identitaires que nous avons pu développer. Le chercheur étant parfois radicalement scientifique et parfois confondu avec les enseignants qu'il observe sur le terrain. Les intentions-mêmes, c'est-à-dire la vie psychique interne, varient et les mouvements de distanciations et rapprochements ne sont pas que sociaux ou externes.

De cette méthode du terrain long découle un statut des données particulier qui s'apparente à un récit d'expérience. On a en effet pu voir comment le carnet de terrain ethnographique (fichier d'informatique mobile dans lequel sont prises les notes quotidiennes sur des événements précis observés et des dires importants qui pourraient être cités, mais aussi 
des analyses hebdomadaires sur ces faits) glissait vers une écriture réflexive, la seconde laissant plus de place aux orientations et finalités du professionnel de terrain dans les traces scripturales.

Le chercheur n'étant pas toujours déclaré ouvertement sur le terrain, et ses intentions étant variables lors de la participation aux événements, le matériau empirique contient plus des notes de travail ou récit d'expérience, se finalisant par des articles ou livres publiés une fois un travail effectué en concertation avec des éditeurs, que les traditionnels entretiens enregistrés et cités en annexes (quoique cela ait été parfois le cas, au début du moins) ou mêmes les photographies et documents filmiques (qui, eux aussi expérimentés, posent des questions d'anonymat et de droit à l'image).

Une synthèse de ces données empiriques donne le statut de récit d'expériences à notre travail scientifique scriptural, c'est-à-dire au matériau pris dans son ensemble. Le statut de ces données est lié au mode de recueil d'information et nous permet de compléter le panorama des méthodes en sciences sociales en liant deux traditions, l'une ethnographique que nous avons apprises à l'École normale supérieure et au Collège de France, l'autre d'écriture réflexive que nous avons développé au Conservatoire national des arts et métiers.

Cet outil méthodologique est ainsi au service de la fabrication de connaissances puisqu'il permet de produire des analyses scientifiques. Ses conséquences épistémiques, et nous retenons les mots de notre mentor Jean Marie Barbier «épistémophilie » et «anthroponomie », sont multiples, comme l'implication dans l'action qui oriente le regard descriptif mais aussi, parfois, les analyses. C'est en croisant les récits d'expériences ou matériau brut (quoique le terme «brut» soit discutable étant donné la formalisation nécessaire de toute écriture) avec les objets de laboratoires et centres d'intérêts des éditeurs scientifiques, qu'ont été produits les documents présentés sous forme synthétique.

Le récit d'expérience croise donc les problématiques classiques d'illusion (biographique notamment) puisqu'il présente un réel mis en forme, reconstruit pour les besoins du laboratoire ou des revues, qui n'échappent pas aux intérêts du moment : intérêts d'auteur et de chercheur dans sa carrière scientifique, intérêts des agents observés sur le terrain qui, parfois, lorsque la recherche est annoncée, tentent de l'instrumenter.

Le récit d'expérience, lorsqu'il est anonyme et discret, débouche le plus souvent sur des entretiens informels, des observations non programmées dont le sens est trouvé a posteriori. Mais il ne s'interdit pas l'approche ouverte où l'entretien déclaré est enregistré 
avec l'assentiment des l'interviewé et où la situation d'observation est provoquée intentionnellement - quoiqu'alors on se laisse moins surprendre par le terrain qui force la pensée exploratoire par ses nouveautés et que l'on ne trouve essentiellement que ce que l'on vient chercher, pour confirmer ou infirmer ses hypothèses.

Notre récit d'expérience est ainsi plus inductif, basé sur les faits bruts que l'on côtoie en permanence, que déductif, cherchant à confronter au réel des idées venues de l'extérieur. C'est cette dimension empirique qui en fait la scientificité et qui lui permet d'être plus ouvert à des littératures multidisciplinaires (sciences sociales, sciences de gestion, sciences politiques, etc.) pour éclairer le fait éducatif. L'inscription de l'abondance de faits dans une littérature scientifique prolixe permet la production régulière d'analyses, publiées régulièrement sous forme de documents de travail, c'est-à-dire avec un statut scientifique hybride.

\section{Résultats de recherche dans les métiers de la formation}

Notre position scientifique et sa méthode d'observation-participante ayant été présentés, détaillons à présent plusieurs des résultats que nous avons atteints dans les sciences de l'éducation et le champ de la formation des adultes en particulier. Nous allons procéder par « objet » de recherches, c'est-à-dire des axes structurants, jetés en avant pas à pas selon les mots de Jean Pierre Boutinet, qui ont guidé nos analyses au cours de nos enquêtes et qui marquent notre appartenance au laboratoire du Centre de Recherche sur la Formation du Cnam et au laboratoire Cirel de l'université de Lille.

\section{Formation : pédagogie et didactique}

Le Centre de Recherche sur la Formation (des adultes) a la formation pour champ principal d'investigation. Ceci correspondait bien à notre terrain d'observation au Ced puisque nous étions responsable de formation chargé d'ingénierie de formation, rattaché à la direction des formations et services. Le dispositif du Conservatoire national des arts et métiers, institution associant des professionnels à des chercheurs notamment par les cours du soir, correspondait bien au cadre de l'étude 
Un de nos premiers mouvements a été de présenter au séminaire dans lequel nous collaborions des éléments d'analyse formelle. En utilisant la méthode, fréquente chez le philosophe Vladimir Jankélévitch, consistant à faire varier des préfixes et suffixes pour éclairer la polysémie d'un étymon, nous avons ainsi présenté plusieurs notions connexes à l'idée de forme. Ainsi, par exemple, l'idée de conformité, avec le préfixe con- désignant le collectif, permet de penser une promotion dans une approche-programme en formation, visant à acquérir tous les mêmes résultats d'apprentissage. De même le suffixe -tion, désignant un processus, adjoint la racine forme, permet de donner l'idée d'un mouvement : mouvement de l'apprenant vers les formes communes (ou résultats d'apprentissage, qualités et compétences) à apprendre, mouvements de ces formes communes d'année à année selon l'actualité scientifique, culturelle et professionnelle. Ceci a constitué notre premier travail sémantique sur la notion de formation au sein même du laboratoire ${ }^{11}$.

Mais comme nous l'avons écrit plus haut, nous avons aussi commencé, du fait de notre terrain, par des lectures sur l'enseignement à distance et par la traduction de la théorie de la distance transactionnelle de Moore. Nous nous sommes réappropriés sa théorie de la formation à distance, avec ses deux axes « dialogue » et «structure » que nous avons insérés dans la tradition du Vieux Continent par un croisement de la pédagogie (au sens d'accompagnement discursif ; nous préférons le terme de pédagogie à celui d'andragogie du fait de l'apport des études de genre) et la méthode (au sens de «learning path » et de didactique). Ainsi, au-delà de l'analyse formelle, nous avons mené des travaux liminaires, en formation des adultes, sur la pédagogie et la didactique.

\section{Pédagogie}

En parallèle de notre thèse, nous nous sommes attelés à la direction d'un dossier scientifique dans la revue Éducation permanente, supervisé par son directeur et validé par le comité de lecture, sur le thème Théâtre et formation. Le lien avec la thèse de philosophie était évident, bien qu'il ne s'agisse pas de théâtre taurin : la méthode était l'observation d'un spectacle et la mise en actes de valeurs; le résultat était les enseignements tirés pour les acteurs et spectateurs. Soit que l'on apprenne en regardant un spectacle et comprenant son message, soit que l'on fasse vivre une langue vivante ou s'identifie à un rôle professionnel en

\footnotetext{
11 Marty O., 2012, Le "projet G. " : une ingénierie de formation. Conception et gestion d'un dispositif d'enseignement (le geste et l'effet). Document de travail pour I'Axe 4 « Organisations et formation » du CRF. <halshs-00776590>
} 
étant soi-même acteur d'un dispositif scénique dirigé, soit, enfin, que les techniques mêmes de la théâtralité comme la modulation de la voix ou le langage corporel nous forment au métier d'enseignant ${ }^{12}$.

Ainsi nous avons étudié les pédagogies actives dont le théâtre constitue un exemple lorsque le formateur met en scène les apprenants qui sont alors acteurs de leur formation. Pour ce faire nous avons dirigé la rédaction de quatorze articles d'universitaires sur les usages du théâtre en formation, dont la moitié, incluant le nôtre, portait sur le genre innovant du théâtre d'improvisation et se rapprochait des jeux de rôles pédagogiques en formation d'adultes.

Le lien entre le théâtre et l'enseignement à distance est devenu celui d'une complémentarité, nombre d'auteurs reconnus du champ s'efforçant de montrer que l'hybridation entre la présence et la distance est nécessaire. Nous avons prolongé ce travail sur la complémentarité dans un livre collectif en analysant un serious game, constituant un jeu de rôle sérieux médiatisé dont nous avons montré l'utilité pour la pédagogie et les limites liées à la distance et la nécessité de le renforcer par des activités similaires en présence ${ }^{13}$.

La pédagogie active en présence a ainsi été prolongée par une réflexion sur les pédagogies numériques. Le serious game présenté dans le chapitre collectif est un premier exemple. Il introduit la notion de théâtre numérique qui constitue un nouvel algèbre éducatif : le dispositif technique est en effet une nouvelle façon de mettre en équivalence des apprenants, de «faire classe » comme disait Philippe Meirieu. Ainsi les participants des jeux sérieux, mais aussi les participants d'une classe virtuelle, sont assemblés comme éléments d'une même formation par l'outil technique lui-même, qui détermine la relation trigonale enseignant-enseignés-enseignements par ses artifices techniques. Enfin, notre terrain étant l'enseignement à distance, nous avons réfléchi aux dynamiques de groupes dans les forums où les apprenants sont les acteurs numériques de leur formation. Nous avons ainsi présenté ces résultats dans une conférence internationale à Séville avec actes ${ }^{14}$ et qui ouvre aussi des

\footnotetext{
12 Marty O., 2012, "La formation par le théâtre : scènes, acteurs et improvisations. Éditorial. » Éducation permanente. p. 56. Documentation française. Paris. $\leq$ halshs-00806539>. L'éditorial présente le travail de recherche dirigé. Il est suivi de mon propre article hybride entre la philosophie et les sciences sociales de l'éducation: Marty O., 2013, "Lumières sur l'improvisation théâtrale : trois usages éducatifs d'une pratique sociale innovante ". Éducation permanente, p. 59-68. Documentation française. Paris. $\leq$ halshs-00806538>

13 Marty O., 2015, "Du métier informatique à l'art de la formation. Une critique épistémologique et esthétique du didacticiel Ammonite. " chapitre dans Cardona Gil Emmanuel, Lemaître Denis (dir.). La modélisation des activités managériales au défi de la formation. Analyse d'un serious game, p. 75-92, L'Harmattan. Collection Action et savoir. Paris. $\leq$ halshs-01187721>
}

${ }^{14}$ Marty O, 2013. "Learning on an online campus. Students team building through online interactions with a LMS. » ICERI2013. 6th International Conference of Education Research and Innovation, Seville. Spain. $\leq$ halshs-00923472> 
perspectives de réutilisation méthodologique dans les Internet studies pour analyser, non seulement la pédagogie, mais aussi le travail d'apprentissage et les dynamiques humaines en ligne. Ce sont les sciences de l'apprendre (à distance) qui sont travaillées par observation directe sur les plateformes.

\section{$\underline{\text { Didactique }}$}

Le deuxième axe que nous avons travaillé est celui de la didactique. Développant les travaux de Moore sur l'enseignement à distance, nous avons rédigé en 2012 document qui croise, dans le schéma de Moore, la pédagogie et la méthode. Nous sommes revenus sur ces travaux et pensons que l'intégration la plus durable dans la tradition de recherche française est celle de didactique et non de méthode (du fait des travaux menés par Jean Pierre Astolfi il y a une quinzaine d'années).

Notre intérêt a aussi porté sur la didactique professionnelle de Pierre Pastré au Cnam. Nos premiers travaux de DEA sur la socialisation d'un employé nouvellement arrivé dans l'organisation se sont ainsi prolongés par l'article précédemment cité sur les professionnalisations à l'ingénierie de formation à distance. Nous avons ainsi observé et analysé comment les acteurs les plus anciens de l'organisation enseignaient leur métier aux nouveaux venus et ce par différentes voies : soit par la formation sur poste de travail (de type compagnonnage), soit par formation préalable au travail par des interventions en cursus de formation (de type enseignements professionnels).

Dans cette lignée, nous avons préparé une intervention pour une conférence de didactique professionnelle, sur la didactique professionnelle de la recherche montrant, pour former de jeunes chercheurs, les spécificités de la multi-appartenance institutionnelle du chercheur en éducation dépendant à la fois d'un laboratoire et de l'organisation éducative qu'il observe. Cette double appartenance est source certes de contraintes et de pluriactivités parfois contradictoires dans certaines circonstances mais aussi d'une ambivalence qui renforce sa valeur (ce qui est fait dans une institution peut-être rétroactivement reconnu dans l'autre) et augmente sa liberté d'action et de recherche. 


\section{Professionnalisation}

Cette seconde didactique, la didactique professionnelle, a été pensée comme une des branches de la professionnalisation. Ainsi Richard Wittorski écrit comment la professionnalisation inclut à la fois la constitution sociétale d'une profession, mais aussi les efforts d'un individu pour augmenter ses compétences dans cette profession et enfin les dispositifs mis en place par les organisations pour développer l'intégration des acteurs dans la profession. La didactique professionnelle peut alors être pensée rétroactivement comme ce troisième cas de la professionnalisation.

Richard Wittorski étant directeur du laboratoire du CRF alors que nous étions dans notre terrain du Ced, nous avons plus particulièrement travaillé sur cet axe de la professionnalisation qui concerne l'apprentissage progressif du métier par les nouveaux entrants. Ainsi dans l'article précité sur les professionnalisations à l'ingénierie de formation à distance, nous avons prolongé un travail sur la socialisation du nouvel employé que nous avions déjà entrepris dans une de nos monographies de Start Up.

L'approche en termes de socialisation, qui relève des sciences sociales et de la sociologie en particulier, nous a permis de mettre en évidence comment la relation entre formateur et formé (en situation de travail comme en formation préalable au travail) primait sur la prise en main des objets techniques. Ainsi, que ce soit l'acquisition du matériel de bureau en Start Up, la prise en main du logiciel de scolarité ou encore de l'outil de classe virtuelle dans l'enseignement à distance, ceci est cadré par une relation de tutorat qui complète l'autoformation, l'augmente et lui ouvre des perspectives. Il s'agit bien d'une professionnalisation anthropotechnique, plaçant l'anthropique avant le technique. La professionnalisation étudiée est plus qu'un dispositif sociotechnique, elle inclut la relation affective, cognitive et développementale entre agent professionnalisant et agent professionnalisé - en agence.

Notre méthode d'analyse organisationnelle permet d'introduire ici l'apport de la professionnalisation à la déontologie et aux valeurs propres au métier. Ainsi, alors que nous étudions la formation du nouveau manager à l'outil de comptabilité analytique, nous voyons comment s'intègre l'importance accordée à la valeur de la rentabilité, que ce soit en actes par la mesure de cette valeur ou dans les discours puisque la référence à l'outil, son mode de calcul et ses résultats servent d'argument dans les discussions avec les enseignants (portant un 
autre régime de justification). La professionnalisation à une déontologie organisationnelle peut ainsi être une piste de recherche pour des développements futurs.

Enfin, l'objet professionnalisation, qui est une direction plus encore qu'un axe de recherche, peut être étudiée en usant des travaux de Jacques Perriault (relatifs au Ced et à l'innovation), prolongés par la notion de catachrèse. Le détournement de l'usage, la différence entre l'intention du concepteur et la pratique de l'utilisateur, peut éclairer la question de la professionnalisation. En effet, que se passe-t-il si, une fois l'agent professionnalisé, il est détourné de son usage pour servir d'autres professions?

C'est une question qui rejoint la multi-appartenance institutionnelle dans la didactique professionnelle du chercheur. Le chercheur socialisé et professionnalisé, compétent dans son travail, ne sert plus directement l'organisation mais est dévoyé, en quelque sorte, par la recherche qui l'amène à d'autres fonctions (c'est ce qui nous est arrivé en devenant enseignant chercheur temporaire à Lille, chargé de cours à distance et dirigeant un mémoire d'ethnographie sur le site du Ced où nous avions exercé). Les particularités d'un terrain dans l'enseignement et des sciences de l'éducation en général est alors la réutilisation des compétences professionnelles de terrain (éducatif) dans la recherche et l'enseignement (ici à distance).

C'est aussi la question d'une professionnalisation de la profession qui peut être travaillée alors que professionnel d'une autre profession : le terrain de l'enseignement à distance est quitté alors que l'acteur est professionnalisé, il ne sert plus directement l'institution d'étude mais contribue, depuis son poste d'enseignant-chercheur, à définir les canons de l'enseignement à distance et donc à aider la profession à se constituer socialement (par des enseignements sur ce qu'elle est). C'est par exemple ce que nous avons fait à l'université de Lille où nous avons écrit notre publication Penser l'enseignement à distance et où nous avons délivré un cours de master d'ingénierie didactique sur ces métiers-là.

\section{Organisations}

L'objet de recherche « organisation » va dans le prolongement des trois monographies d'organisations que nous avions réalisées en Start Up au préalable de l'étude sur 
l'enseignement à distance. Nous avons donc réfléchi à la notion d'organisation, et nous l'avons défini provisoirement comme un processus dynamique de coopération de plusieurs organes professionnels, ou plusieurs métiers, ayant chacun leurs compétences et déontologie.

Ceci nous a permis de penser plusieurs terrains en plus de celui du Ced. Nous avons indiqué que notre doctorat avait été écrit alors que nous étions cadre dans un organisme de formation professionnelle continue. Nous avons retraité l'expérience accumulée dans cet organisme de formation lors d'une conférence invitée en Sorbonne, pour le doctorat professionnel en sciences sociales de Paris $5^{15}$. Cette conférence invitée a été l'occasion de comparer le terrain dans l'enseignement à distance à nos connaissances empiriques de la formation professionnelle continue (où nous avions exercé deux années comme cadre durant notre thèse). Nous en avons retiré un schéma heuristique dessinant les lignes de forces du champ de la formation professionnelle continue privée. Ainsi sont assemblés en une vue systémique les fonctions des ressources humaines des entreprises consommatrices de formation, les formateurs indépendants vendant leur compétences sur le marché et les intermédiaires constitués par les organismes de formation - auxquels s'associent des instances de légitimation qu'ils s'approprient : éditeurs professionnels, revues, organisateurs de prix appartenant au même groupe financier.

Nous sommes ici au niveau d'un ensemble d'organisations faisant système. À ce niveau, nous avons aussi opéré un retour sur l'institution éducative Edhec au cours de deux conférences en colloque international :

- Olivier Marty. 2013. « Studying (in) a French grande école. Combining mobility, motives and mobilization ». Colloque HEM. Higher Education and Mobilities, Dec 2013, Grenoble, France. 〈halshs-00831980v2>

- Olivier Marty. 2013 «Étudier (dans) une grande école française. À la croisée du modèle américain néolibéral et des valeurs éducatives du vieux continent ». Colloque international. Les questions vives en éducation et formation : regards croisés France-Canada, Jun 2013, Nantes, France. <halshs-00831524v2>

Ces deux conférences, une en français et l'autre en anglais, portent le même titre principal. Ceci ne doit cependant pas leurrer le lecteur : comme l'indique le sous-titre les contenus sont différents. Il s'agit en fait de la même démarche : étudier une grande école en étant inscrit

\footnotetext{
${ }^{15}$ Marty O., 2013, « Les métiers innovants de la formation. Le champ de la formation professionnelle et l'ingénierie de formation à distance ». Les métiers innovants de la formation, Paris. France. <halshs-01007448>
} 
comme élève (c'est le sens de la parenthèse dans le titre); mais les résultats sont différents. Dans la conférence grenobloise nous avons voulu montrer le parcours historique de l'institution et sa mobilité vers une reconnaissance internationale. Dans la conférence nantaise nous nous sommes intéressés à la valeur de la liberté sous-tendant sa politique, reprenant ainsi les thèmes premiers que nous avions travaillé en philosophie politique à Toulouse.

La notion d'organisation telle que nous l'avons travaillée est donc proche de celle de champ bourdieusien (les organisations de la formation professionnelle continue et leurs instances de légitimation symbolique) et des approches institutionnelles (l'histoire d'une grande école sur un siècle, la valeur de la liberté dans ses orientations éducatives influencée par le Nouveau Monde). Dans les deux cas nous avons retraité une expérience passée avec le filtre des questions de recherche propres aux sciences de l'éducation.

Ceci a été complété par des travaux d'encadrement de mémoire à l'université de Lille. En effet, nous avons dirigé le mémoire d'un ingénieur en reconversion professionnelle qui s'était inscrit dans le master ingénierie pédagogique et multimédia. Ce mémoire traitait de la mise en place d'une école de formation interne au sein de la police judiciaire. Il analysait les controverses, positions et arguments, des différents acteurs impliqués dans la création de l'école au sein de l'organisation.

\section{Activités}

Nous nous sommes aussi efforcés de travailler des objets complémentaires à celui des organisations. Ainsi nous pouvons présenter Le droit chemin : une méthode. Acte, activité et action d'apprentissage qui est le support d'une conférence. Ce support d'intervention oral est modeste dans ses dimensions, il est cependant important car il propose une théorie insérant l'activité dans un continuum allant de l'acte à l'action en s'appuyant sur le cas concret des agents du Ced s'ingéniant à concevoir une formation. Ainsi la répétition rythmée d'actes fait l'activité et le sens donné par les valeurs transforme l'activité en action.

Dans la continuité de ce travail, nous pouvons relire une grande partie de nos travaux comme un effort pour penser les activités et l'action en général. Cette réflexion s'est faite selon plusieurs cadres d'analyses classiques qui nous permettent de regrouper nos résultats au fil de notre parcours : 
- La poétique ou l'action créative, que ce soit pour l'innovation en Start Up ou les TICE, nous avons insisté sur la dimension nouvelle et créative des actes observés.

- Le drame ou l'action sociale, collective et coactive, comme nous l'avons développé dans notre recherche sur les usages du théâtre et des jeux de rôles en éducation, en grande école notamment.

- L'ergologie ou la réflexion sur l'action productrice, l'analyse du travail. Ainsi on peut relire nos travaux, depuis les Start Up et la construction des pirogues, jusqu'au terrain dans l'enseignement à distance, comme une ergologie. Même notre thèse, réflexion sur les valeurs d'un métier, est une analyse des référentiels internes de cette pratique-là, basée sur une observation d'une éthique professionnelle en actes.

- La praxéologie, réflexion plus ancienne encore sur les pratiques, qui est notre objet au sens large, quoiqu'allant en se concentrant sur les pratiques des métiers de la formation. $\mathrm{Du}$ fait de l'analyse organisationnelle des pratiques déontologiques, nous préférons le pluriel praxéologies. Pluriel qui est double : il s'agit d'étudier les logiques sur les pratiques des différents acteurs, éventuellement en conflit ou en phase d'harmonisation.

Cette praxéologie est une logique de la pratique non nécessairement pragmatique. Ainsi, pour reprendre la discussion de Richard Wittorski sur les liens entre la réflexion et l'action, nous proposons une réflexion de l'action, à la fois sur (c'est son objet) et pendant (c'est son mode) l'action, mais pas pour l'action vers laquelle elle convergerait : au contraire la réflexion est parallèle à l'action, voire en libère, car elle est légèrement divergente. Nous nous démarquons ainsi des sciences de gestion visant à augmenter la productivité et nous nous inscrivons dans le champ du critique et de la compréhension sociologiques, du récit historique, de la prospective éducative.

Nous ne défendrons pas ici, car ce n'est pas le propos de cet ouvrage, un lien entre éthique et ethnique, autour de l'action collective, la morale ou les mœurs propres à un ensemble humain. Ceci pourrait toutefois constituer un fondement anthropologique solide.

Notre pensée de l'activité est certes une pensée noétique mais elle appartient à la logique scientifique du dicible et du réfutable, ouverte à la contradiction et au dialogue poppériens. Ce alors que, paradoxalement, elle relève d'une épistémologie scientifique constructiviste : la réfutabilité vise à corriger les constructions collectives pour mieux les insérer dans les paradigmes existants et la science normale, sans pour autant prétendre les rapprocher d'un réel absolu dont l'ontologie serait inaccessible. 
Nous présentons ainsi plusieurs de nos résultats que nous pensons féconds et qui pourraient être développé dans une direction de travaux :

- La dimension du temps, parce qu'elle est intrinsèque à l'action mais aussi à sa mise en récit scientifique.

- La dimension de l'espace dans ses repères physiques visibles et culturels, parfois cachés.

- La dimension coactive des drames et ce qui en découle : le verbe et les sonorités des interactions colloquiales. La dimension linguistique invite à explorer les cadres sémantiques des sémiologies non occidentales à l'ère postcoloniale, comme nous avons commencé à le faire avec l'arabe littéraire (la racine d-r-s dans l'analyse du travail éducatif) mais qui appelle à une extension (nous nous intéressons à une des langues de l'Asie du Sud : l'ourdou). Il s'agit de pouvoir penser l'action éducative en dépassant le cadre helléno-latin, voire germanique, grâce à des mots à la polysémie originale et l'étymologie complexe.

- La dimension sociale et psychique : puisqu'il s'agit de personnes, comprendre au-delà des dynamiques de groupe, la genèse de leur personnalité et de leurs valeurs d'acteur, la clinique de leur activité, les facultés cognitives activées : la sociologie et la psychologie dans leur union autour de l'action et non uniquement leur intersection.

\section{Communication}

Notre intérêt principal pour cet objet portait sur le vocabulaire : les mots et leur sens, la sémiologie et la sémantique. Ainsi, dans nos monographies d'organisations innovantes comme dans notre travail ethnographique en Guyane, nous avions déjà travaillé sur le « vocabulaire des indigènes » et leur interprétation dans la langue scientifique. Ceci rejoignait, dans le vocabulaire de l'analyse des activités éducatives, la définition du savoir par Jean Marie Barbier, comme énoncé valorisé socialement - ce qui ne va pas sans introduire un relativisme auquel nous adhérons en majeure partie. 
Ainsi nous avons travaillé au vocabulaire utilisé au $\operatorname{Ced}^{16}$ pour décrire les apprenants qui étaient considérés différemment par les métiers coopérant: la scolarité parlait « d'inscrits », les enseignants «d'élèves », la direction commerciale de «clients ». Cette même profusion de statuts se retrouvait dans le vocabulaire des grands établissements distinguant différents parcours : les «élèves » fonctionnaires, les «étudiants » universitaires ou les «stagiaires» issus du monde professionnel. La description de ces vocables, et les tentatives de définition scientifique précises de termes stables (ainsi notre travail autour de «l'apprenant» comme celui qui prend une forme, à la fois actif dans son apprentissage et collégial dans ses compréhensions), nous a permis de prendre de la distance avec les discours des personnels étudiés durant le terrain long. C'est un travail initiatique qui, d'emblée, place le chercheur en observateur de l'organisation qu'il intègre et le sensibilise aux conflits sur les valeurs, idées et appellations des réalités au sein du milieu observé.

Ce travail sur le vocabulaire a été repris et élargi par des analyses intégrant plusieurs langues dans une perspective étymologique. Tout d'abord les langues mortes (grecque ou latine) nous ont servi à explorer des champs sémantiques, soit en faisant varier les préfixes et suffixes comme précédemment montré (dans l'analyse formelle du terme « formation »), soit en rapprochant des mots à la racine identique (ainsi, dans le programme de recherche sur le doctorat de Françoise Cros : «docteur », « document », « éducation ${ }^{17}$ ). Et, dans un second temps, nous nous sommes intéressés aux mots exotiques, venant d'autres aires culturelles majeures en interaction avec l'occident. Nous avons ainsi exploré la racine de l'arabe littéraire D-R-S au cours d'une conférence au Cnam en montrant comment le rapprochement lexical entre darsoun (la leçon), madrasa (l'école) et moudaris (le professeur) permettait de remettre en perspective un héritage grec centré sur les mots pédagogie, méthode, didactique, précisé par les latins sur la relation éducative, le parcours d'apprentissage ou encore les signes enseignés. Nous pensons que ce travail peut être prolongé en étudiant les polysémies pour comprendre les échos que peuvent avoir, selon la langue, une notion - car elle véhicule, par une appellation commune, d'autres notions dans l'inconscient linguistique que l'on s'efforce de mettre à jour.

\footnotetext{
${ }^{16}$ Marty O., 2012, "Le Ced : administration ou industrie éducative ? Les valeurs et la culture éducative d'une institution en formation ". <halshs-00777012>. Ce document est une sociologie de l'institution éducative du Ced, orienté et guidé par Jacques Perriault, fondateur du premier laboratoire du Ced (le LABIC), alors au CNRS lorsqu'il nous prodigua ses conseils.

${ }^{17}$ On se référera ici utilement aux notes de base de page supra, que l'on peut reprendre en disant que si l'on se fonde sur l'hypothèse d'un bien commun linguistique indo-européen, il est possible de faire remonter doceo à dekhna (montrer) et dekhana (faire montrer) par la racine encore vivante dans l'hindi dekh rapprochement alors l'éducation du signe de l'enseignement.
} 
Au-delà du vocabulaire, des indigènes, scientifique ou étymologique, nous avons repris nos cadres d'analyses issus de nos livres en science sociales - ce que nous avions montré dans nos livres Le sons et le sens et Klikoo.com : la notion de parole distribuée fait notamment l'objet d'un graphique sous forme de carte mentale pour montrer les effets subliminaux en situation éducative d'association d'un mot à un interlocuteur. Ainsi les adjectifs peuvent être aisément associés à tel ou tel interlocuteur par un regard ou une adresse lors de la prise de parole en groupe, ce qui produit des effets sur la structure évanescente du groupe. De même pour les articles, voire les noms communs.

Enfin l'objet « communication » a été travaillé au détour d'un document de travail sur le marketing de la formation. Il s'agissait de comprendre la politique de valorisation des formations du Ced par l'intermédiaire d'un site Internet («webzine ») présentant des parcours atypiques d'étudiants (sportifs de haut niveau, voyageurs, artistes itinérants, etc.). Nous avons décrit cette communication en nous inspirant des travaux de Patrice Flichy sur les imaginaires de l'Internet (l'audiovisuel informatique se prêtant bien à cette notion d'imaginaire développée par le philosophe Gaston Bachelard dans ses analyses poétiques sur les éléments). Ici la communication est réfléchie et travaillée autour d'un artefact technique qui présente les usages atypiques de dispositifs de formation de l'institution. C'est une vision plus générale que le souci du vocabulaire et de sa distribution sociale qui constituaient nos premiers résultats.

\section{Identité}

Après avoir présenté nos principaux objets de recherche au Conservatoire national des arts et métiers, montrons les résultats liés à un contrat d'attaché temporaire d'enseignement et de recherche à l'université de Lille 1. Nous intégrions alors un nouveau laboratoire avec des objets nouveaux. Nous allons présenter l'objet «Conception» mais, avant cela, appuyonsnous sur l'objet « identité » qui est commun aux deux laboratoires, notamment en la personne de Mokhtar Kaddouri qui a appartenu aux deux et qui nous a accueilli dans son séminaire lillois. 
Nous avons en effet pensé l'objet « identité » en référence à l'étymon idem qui indique à la fois une continuité dans le temps (identique à soi-même) et à la fois une adaptation à l'environnement (identique aux autres). Le terme d'identique n'interdisant pas un positionnement social différencié : chacun ayant sa propre identité. Et l'environnement étant changeant, cette identité complexe est ainsi amenée à évoluer puisque l'acteur est pris dans des contraintes sociales extérieures nouvelles qui génèrent des évolutions psychiques internes plus lentes.

C'est ce que nous avons présenté autour de «l'identité du chercheur » en rappelant le modèle de l'analyse des distances et des variations entre l'identité scientifique et l'identité de terrain, les concessions et les compromis faits pour maintenir cette double appartenance le temps du terrain. Ces travaux prolongeaient une conférence à Prague en sciences de l'éducation, spécialisée sur les terrains longs en enseignements ${ }^{18}$.

La discussion des travaux de Mokhtar Kaddouri porte alors sur les notions d'engagement et de valeur, voire de transition professionnelle. En effet, l'engagement sur le terrain, qui est signe scientifique important, peut ralentir l'engagement dans la communauté d'enseignants, profession souvent associée à la recherche. Des valeurs contradictoires peuvent apparaître et il faut pouvoir penser la transition entre les années de terrain et le retour à son poste de travail académique. Ceci bénéficie en partie de notre formation initiale en ethnographie au Collège de France et nombreux sont les anthropologues qui, de Mauss à Bazin, pensent ces allers-retours avec le terrain et ses conséquences identitaires. L'apport des concepts du professeur Mokhtar Kaddouri permettant de renouveler les termes de l'analyse sur ce sujet classique, notamment par la transition professionnelle d'une identité à l'autre (le changement de catégorie à l'Insee n'allant pas sans conséquences matérielles et il peut être important d'étudier la préparation à ces transitions par la formation initiale complexe évoquée ci-avant et présentant déjà ces types de contradictions).

\footnotetext{
18 Marty O, 2013, «An epistemology of distance. Ethnography of training activities: engineering and management in higher education organizations. International Conference. Ethnographies of higher education: researching and reflecting "at home"», Czech Republic. <halshs-00833739>
} 


\section{Conception}

Le travail sur l'objet «conception» est associé à celui d'Annie Jézégou et à ses recherches discutant les apports de Moore à la théorie de l'enseignement à distance. Nous avions commencé à travailler sur ce domaine-là, de la «conception », de l'ingénierie et de l'innovation, dès les monographies d'organisations pré-doctorales puisque nous avions défini les Start Up comme un pari sur une innovation, innovation qui était au cœur de leur modèle économique. Ce dans la lignée des travaux de Patrice Flichy en sociologie de l'innovation. Il faut cependant ici différencier le produit d'une conception qui est une innovation mise sur le marché par une petite organisation d'une part, et le processus d'innovation en tant qu'objet d'étude: la fabrique de l'innovation qui a été l'objet de nos travaux en sciences de l'éducation.

Ainsi, concepteur de formation dans le privé puis chargé d'ingénierie de formation au Ced (c'est-à-dire en charge de la conception de produits de formation à distance d'envergure nationale, conventionnant plusieurs universités ou écoles partenaires), nous avions un poste d'observation pour cet objet d'étude. Jean Marie Barbier nous avait indiqué une différence fondamentale entre les travaux scientifiques portant sur l'objet «conception » ou ingénierie d'une part; et les travaux professionnels d'ingénierie.

Ainsi nous voulons présenter séparément un projet d'ingénierie en lien avec le ministère de l'enseignement supérieur et de la recherche : celui d'ingénierie d'un diplôme national de licence basé sur l'enseignement à distance de nouvelle génération (les Moocs) qui a fait l'objet d'une audition et d'une collaboration de la chef de service de l'enseignement supérieur au ministère (document de travail initial rendu public sur HalSHS, tout en préservant la confidentialité de l'apport ministériel) ${ }^{19}$. Même si la frontière entre la science et les techniques en éducation sont ténues, il nous paraît important de mentionner ce document car il est un produit de l'activité d'ingénierie qui peut servir de base, dans sa description, pour mieux comprendre les processus de conception (ce que nous avons fait en relisant, ci-dessus, l'évolution des travaux dans le terrain long en enseignement à distance).

Le document sur «le projet G. » décrit plus en détail et scientifiquement le processus de conception d'un produit d'ingénierie et se veut un complément scientifique à la technique de

\footnotetext{
${ }_{19}$ Marty O, 2016,. Licence ouverte. Utilisation des Cloms pour obtenir le grade de licence. [Rapport Technique] Direction générale de l'enseignement supérieur et de l'insertion professionnelle. Paris. $\leq$ halshs-01329191>
} 
gestion de projet. C'est un document de travail sur le temps en formation : non pas le temps du récit scientifique ou indigène qui linéarise dans un propos situé les étapes du projet. Nous nous écartons de la reconstruction linéaire de l'histoire du projet avec lissage des aléas passés et prolongation dans le futur de la ligne droite nouvellement tracée, gommant toutes les lignes brisés et les tentatives manquées que relèveraient une clinique de l'activité. Nous optons au contraire pour un modèle cyclique s'appuyant sur les temps ou moments-forts (c'est-à-dire valorisés) qui scandent l'activité et le projet d'ingénierie : demande initiale, recherche d'information, travail collectif, remise des rendus de dispositif, ouverture au public, évaluation, amélioration. C'est une analyse des rythmes de ces moments qui reviennent régulièrement, selon des boucles plus ou moins longues, et non des lignes de narration trop réductrices. Ceci est valable quel que soit le niveau de conception : ingénierie pédagogique des enseignants, ingénierie de formation des managers ou ingénierie institutionnelle des décideurs (certains préfèrent le terme d'ingénierie sociale). Seul l'empan temporel change, avec le rythme cyclique qui se ralentit : plus on monte dans la hiérarchie, plus la maturation est longue et les conséquences durables. Les étapes sont plus distantes et ont une portée organisationnelle avec cascade hiérarchique plus importante. Il en va ainsi du support de conférence $^{20}$ sur l'industrialisation des classes virtuelles dans l'enseignement à distance qui décrit une ingénierie relativement brève à l'échelle individuelle et d'une formation (processus d'un an dans le document) mais qui s'installe progressivement depuis une dizaine d'années dans les différentes sphères de la formation - sans que ce mouvement sociétal ne soit encore réifié par une décision capitale.

À l'inverse, on retrouve ce processus au grain le plus fin de l'analyse : une conception individuelle ou l'ingénierie d'un projet d'autoformation repose non plus l'idée d'une formation continuée (prolongeant la formation initiale) mais d'une formation réinitialisée (revenant en boucle sur des apprentissages de base pour les renouveler). Notre propre parcours d'autoformation par licences multiples (diplômé avec mention : sciences politiques, philosophie, sociologie / formation dans les dispositifs à distance sans inscription en scolarité : sciences de l'éducation, psychologie, arts plastiques) montre cet effort de réinitialisation régulier pendant une vingtaine d'années, de recyclages. Ce alors que la recherche constituait une formation scientifique continuée, linéaire et elle aussi cumulative.

\footnotetext{
${ }^{20}$ Marty O., 2013, "Industrializing virtual classrooms in distance education. How to measure added value of a training ? » ICERI2013. 6th International Conference of Education Research and Innovation, Spain. <halshs-00923473>
} 


\section{Apprentissages}

L'idée d'une conception de sa formation, l'ingénierie personnelle de son autoformation, nous amène à l'objet « apprentissages ». Cet objet est important pour l'équipe «Apprenance » de Philippe Carré étudiant notamment le rapport au savoir. Notre approche du rapport au savoir ne porte pas sur les apprenants défavorisés (du fait de leur capital culturel voire de leur parcours de non scientifique selon les termes d'Olivier la Verganas) mais au contraire sur le personnel de l'enseignement à distance. Ce personnel, hautement formé, légitimé par des titres académiques et une position professionnelle est, selon nous, plus dans une logique bourdieusienne de production innovante de savoirs que de reproduction de savoirs. Certes la production n'est pas entièrement libre et est contrôlée par les pairs, selon les règles de disciplines et les possibilités d'expression, mais elle place le professionnel de l'enseignement dans une situation de source en amont et non de récipiendaire en aval du flot d'énoncés valorisés. Ceci étant relativement complexe puisque la source productrice se régénère en puisant dans une masse de connaissances accumulées dans son parcours souterrain et par son réseau. Le producteur de savoirs est aussi un reproducteur, voire un transformateur et ce qu'il produit n'est valable que dans un contexte culturel donné et l'appareil académique qui le légitime en le plaçant dans une position sociale haute.

Nous avons ensuite travaillé l'objet «apprentissage » en nous référent, au-delà de la littérature sociologique, aux sciences de l'apprendre en essor et faisant l'objet de plusieurs manuels anglophones. Le passage à Lille nous a notamment permis de découvrir les travaux d'Albert Bandura, psychologue devenu sociologue traduit en français par les chercheurs de Paris 10 et ceci s'est concrétisé par un support de conférence ${ }^{21}$ au colloque E-formation sur les effets cognitifs d'une technique de médiatisation : l'influence de l'écriture numérique sur l'apprentissage. Nous y avons montré l'importance de la redondance dans ce que nous appelons le genre littéraire du «guide de formation » (c'est-à-dire le livret de l'étudiant), facilité par le dispositif informatique qui place l'information au bon endroit et au bon moment selon plusieurs appareils de lectures encastrés (notre étude portait sur Moodle et comparait les répétitions d'information dans le panneau calendrier, l'arborescence des cours et le forum des nouvelles).

${ }^{21}$ Marty O, 2015, "L'écriture numérique du guide de formation : Quelle influence sur l'apprentissage des étudiants ? » Colloque : E-Formation des adultes et des jeunes adultes, Lille, France. $\leq$ http://www.trigone.univ-lille1.fr/eformation2015/>. $<$ halshs-01181339> 
Élargissant nos observations de terrains, nous avons cherché à définir l'apprentissage en nous basant sur les travaux de Nietzsche (qui étaient centraux dans notre thèse de philosophie) et les études nietzschéennes (trois livres occidentaux portent sur l'éducation chez ce philosophe, ainsi qu'une vingtaine d'articles, comme nous sommes en voie de le faire reconnaître par la communauté de sciences de l'éducation française). Nietzsche pense l'apprenant avant l'éducateur, préfigurant ainsi dès le $19^{\text {ème }}$ siècle John Dewey puis la «bolognisation » de l'enseignement supérieur. Sa philosophie nous incite ainsi à faire le lien politique entre les sciences de l'éducation et les catégories juridiques en vigueur. Nous avons alors réfléchi à la place de l'apprenant (en situation active de prendre la forme d'un programme) dans les catégories de Bologne : le formel, l'informel et le non-formel par exemple ; abondamment promu par l'agence européenne sur la formation des adultes (Cedefop). Dans un support de conférence de cette période sur le dispositif d'e-portfolio ${ }^{22}$, nous avons mis en avant le concept de «formalisation» pour synthétiser les différents moments d'apprentissages (formels, non formels et informels) dans une composition ad hoc mettant en valeur les résultats d'apprentissages (learning outcomes). Nous avons ainsi une préparation de la « reconnaissance » des acquis éducatif par le public visé par le média.

Si penser scientifiquement les catégories juridiques est une activité classique en sciences politiques, c'est cependant relativement périlleux dans le sens où les instances politiques sont sources de financement (ANR, ERC) et donc influencent subrepticement par leurs demandes (dans leurs propres termes et avec leurs propres catégories) le résultat de la recherche. C'est aussi une façon d'anticiper les réalités institutionnelles car les catégories juridiques, réifient la société, soit en la préfigurant par une initiative politique soit en clarifiant les mœurs. C'est par exemple ce qui s'est observé dans le dispositif de France Université Numérique mis en place par la loi de 2013 et réifiant l'enseignement supérieur de nouvelle génération : les cours en ligne ouverts et massifs.

Une autre approche institutionnelle des apprentissages porte sur le glissement du vocable juridique de «qualification» vers «compétences ». Le premier renvoi à la qualité définissant qui est le formé à l'issu de sa formation et donc les fonctions qu'il est à même d'exercer. Le second, plus précis et venu d'outre-Atlantique, porte sur le faire et non l'être : les compétences ou verbes d'action en situation (c'est-à-dire avec leur objet et compléments

\footnotetext{
22 Marty O, 2013, "Digital skills' portfolio: formalizing the informal in computer learning. An ethnography of distance education engineers. " Document de travail. SSRE2013 Annual Conference. Integrating formal and informal learning. Lugano. 2013. <halshs-00814233v2>. Le document était accepté pour présentation en conférence en Suisse mais nous n'avons pu nous y rendre du fait d'un conflit d'emploi du temps avec notre terrain.
} 
circonstanciels de lieu, de temps et de manière), voire en 2019 une préférence ministérielle pour les substantifs, qui décrivent ce que pourra accomplir le formé. Nous pensons que ce glissement de l'être vers le faire est ambivalent: soit que l'on change radicalement de paradigme (l'action avant le statut), soit que l'on pense que l'action prolonge l'être et le précise ( «je fais ce que je suis », renversant l'adage existentialiste selon une éthique antique). C'est le sens de ce que nous avons co-construit avec l'étudiant dont nous avons dirigé le mémoire de master lillois sur le sujet « qu'est-ce qu'une compétence ?».

Nos apports à l'objet «apprentissages » ne se limitent cependant pas à une pensée politique sur le vocable juridique (de droit commun) des pratiques éducatives. Nous avons en effet tenté de remettre en vigueur certaines étymologies et avons retrouvé les mathématiques, substantif issu d'un verbe grec signifiant apprendre. Nous avons ainsi opéré la variation lexicale autour «d'apprendre »: prendre, comprendre, appréhender (dans sa polysémie surprenante : s'emparer et s'effrayer); et cette variation nous a amené à réfléchir à la notion fondamentale de métamorphose en formation, c'est-à-dire de transformation au cœur de l'apprentissage. La revivification d'autres langues a aussi permis de reprendre la hiérarchie platonicienne des sens : la vision primant sur l'audition, l'odorat, le toucher et le goût ; ce qui est le sens du mot «idée » (apprendre des idées) devenu « forme » en latin, et concurrençant, à l'ère de l'audiovisuel, la notion, reconnue dans la discipline des sciences de l'éducation, de « savoir ». Ici une légitimation comparatiste peut être effectuée avec une autre civilisation : le sanskrit et les «védas» qui désignent aussi des visions, et non des saveurs, comme principales sources de connaissances dans leur gnose contemplative.

\section{Valeurs}

La terminologie des apprentissages en termes de formes et d'idées permet de retrouver nos travaux de thèse de doctorat sur l'objet « valeur » (le sous-titre de notre thèse est « Les valeurs du combat selon Frédéric Nietzsche») ainsi que ceux du laboratoire Cirnef de l'université de Rouen. Nous avions en effet, durant notre travail de philosophie, cherché à comprendre la genèse des valeurs pour un individu : l'affection pour certaines idées devenant des valeurs donnant sens à son action. Notre directeur de thèse Francis Wolff avait élargi cette définition en indiquant les valeurs logiques, éthiques et esthétiques fondant notre travail, 
ayant chacune leur propre mode générique. Ce qui nous avait amené à écrire ceci dans notre thèse de doctorat (p.197-198) ${ }^{23}$, sur les valeurs fondées par la métaphysique nietzschéenne :

« De l'éthique à l'esthétique : l'art exprime les valeurs

$(\ldots)$

Nous avons vu que Frédéric Nietzsche concevait le réel comme une lutte de forces qui s'agrégeaient en complexes et cherchent à s'étendre. Pour le philosophe, il n'y a pas d'unité fixe (de sujet ou d'objet) et tout est en lutte perpétuelle : les forces s'opposent et s'agrègent, cherchent à dominer d'autres forces. Le réel est en perpétuel devenir ${ }^{24}$.

Le philosophe Martin Heidegger, dans son commentaire de Frédéric Nietzsche, rapproche cette conception métaphysique du conseil moral : "Deviens qui tu es"25 : il n'y a pas d'individu fixe, la personne est en mouvement et en perpétuelle transformation. Tout au plus peut-elle suivre une direction qui lui donne sens.

Dans le deuxième tome de son ouvrage consacré à Frédéric Nietzsche ${ }^{26}$, Martin Heidegger analyse les valeurs comme relatives à l'augmentation de la puissance de celui qui les institue - comme nous l'avons vu dans l'introduction sur le concept de valeur. A de la valeur ce qui sert au développement de la personne qui cherche à devenir ce qu'elle est. (...)

Tout ce qui entoure l'individu-œuvre-d'art est alors évalué en fonction du mouvement de croissance qui le porte : seuls ont de la valeur les objets qui servent l'œuvre. L'art va bien dans le sens du réel : il l'amplifie et lui donne sens par des actes d'évaluation. (...)»

On retrouve ainsi clairement dans ce premier écrit doctoral sur l'objet « valeurs » les fondements de ce que nous allions travailler en sciences de l'éducation : la confrontation de valeurs entre différents corps (de métier en organisation). Ainsi, la réception française des

23 Accessible en ligne sur http://www.theses.fr/2011PA100067

24 Martin Heidegger, 1971 (1961), Nietzsche, tome 1, p. 426. Gallimard. Bibliothèque de philosophie, Paris.

25 Martin Heidegger, 1971 (1961), Nietzsche, tome 1, p. 16. Gallimard. Bibliothèque de philosophie, Paris.

26 Martin Heidegger, 1971 (1961), Nietzsche, tome 2, p. 83-85. Gallimard. Bibliothèque de philosophie, Paris. 
ouvrages de John Dewey (notamment par le commentaire récent d'Alexandra Bidet) laisse une place majeure à la notion de «valuation", mise en valeur ou valorisation, de son environnement et pratiques. Après avoir acquis les valeurs déontologiques de son métier par la professionnalisation, l'acteur éducatif participe à la professionnalisation des impétrants en imposant des valeurs qui lui servent directement. C'est là une réutilisation scientifique de propos philosophiques du $19^{\text {ème }}$ siècle (Nietzsche avant Dewey), alliant les objets «professionnalisations » et «valeurs », et qui est une contribution à l'histoire des idées en éducation. 


\section{Une institution industrielle de l'enseignement à distance}

Après cette introduction présentant la méthode anthropologique, le terrain long en institution éducative et quelques résultats condensés par objets de recherche et appartenance aux laboratoires, les chapitres suivants vont regrouper sous une forme originale des travaux inédits. Le mouvement sera d'abord de présenter l'institution étudiée, par un document unique sur sa trajectoire historique et ses valeurs. Puis de montrer notre entrée particulière dans cette institution par le «management» de dispositifs d'enseignement, au cœur de ses activités. Ceci sera l'occasion de réfléchir sur les catégories indigènes de sciences de gestion utilisées au quotidien: la finance pour le calcul de la valeur d'une formation, la mercatique pour l'image de marque ou encore la gestion de projet et ses a priori temporels. Ceci nous amènera à préciser le métier de l'enseignement étudié : l'ingénierie de formation et ce que nous avons nommé l'ethnie des ingénus qui est au centre des enseignements à distance. Nous verrons ainsi son engin propre (les théâtres numériques et autres plateformes d'enseignement) et ses génies associés (pédagogie collective, art relationnel fondant ce métier). Ceci sera analysé au fil de descriptions de projets d'ingénierie particuliers. De-là nous tirerons plusieurs apports complémentaires, esquissés dans le chapitre sur nos résultats, aux théories de l'activité en formation et à celles de l'éducation en général.

Mais commençons par le tableau institutionnel présentant l'organisation étudiée. La description de terrain nous permet de saisir les réalités sociales et éducatives du Centre d'éducation à distance (Ced), où nous avons effectué notre recherche. Et de voir notamment la transition d'une culture administrative vers une culture industrielle et commerciale. Nous montrons les valeurs (intérêt général, bien commun, service à l'apprenant) portées par la langue et les habitudes ainsi que la valorisation comptable et numérique des activités de formation en pleine évolution. Ceci nous permet d'esquisser une vision comtienne de la trajectoire sociale de l'institution.

Le tableau institutionnel est donc celui d'une organisation qui évolue lentement depuis une logique administrative vers une logique industrielle. Avec une transformation profonde de ses valeurs qui se manifeste dans le recrutement de ses agents comme dans son vocabulaire et ses scènes quotidiennes (vêtements, modes d'interactions, arguments recevables dans une controverse). Présenter ainsi l'institution nous permet d'annoncer notre propre entrée pour 
l'étude qui doit une grande partie aux sciences de gestion qui y prennent de la place et que nous nous efforcerons de réfléchir en action.

\section{Le Ced : administration ou industrie éducative?}

Le Centre d'éducation à distance (Ced) est une jeune institution : créée en 1939, fille de la troisième République ${ }^{27}$, elle a fait ses classes sous les quatrième puis cinquième Républiques, par de nombreuses modernisations et changements d'appellation. Fascicules papier, téléphone, télévision, minitel, Internet,... l'organisation expérimente, puis industrialise ${ }^{28}$, les nouvelles technologies éducatives. La formation du Ced a donc été progressive.

Aujourd'hui la forme juridique de l'institution est celle d'un Établissement public administratif (Epa) mais se pose la question d'un éventuel passage au statut d'Établissement public industriel et commercial (Epic). Avant le Ced, France Telecom - et même Renault, dans le sens inverse - ont connu cette transformation de l'administration vers l'industrie et le commerce. Ceci est plus qu'un simple changement de droit : dans les faits, c'est toute une culture d'action $^{29}$ qui doit évoluer, avec le sens que les hommes et femmes donnent à leurs actions - leurs valeurs. Quelles sont les valeurs du Ced et en quoi leur devenir est-il signe de l'évolution historique de l'institution?

Nous entamerons notre propos par une analyse de l'évaluation du Ced: pour qui l'organisation a-t-elle de la valeur ? Ce sera l'occasion de montrer les différentes logiques quelle sert, et leurs valeurs différenciées : intérêt général, bien commun, service à l'apprenant. Puis nous nous appuierons sur un matériau issu d'observation participante dans deux sites : Vanves, en région parisienne, lieu historique puisqu'ancien bureau du recteur ; et le parc du Futuroscope, à Poitiers, siège actuel et moderne de la direction générale. C'est le quotidien des employés qui nous permettra de comprendre les changements humains sous-jacents à l'évolution statutaire potentielle : l'hybridation des cultures publique et privée qui se traduit par une évolution linguistique et une révolution numérique. Nous verrons ainsi les mutations

\footnotetext{
${ }^{27}$ Nous nous référons l'histoire produite par l'institution elle-même et disponible sur son site Internet. Nous en préservons toutefois l'anonymat à grands traits.

${ }^{28} \mathrm{Au}$ sens de production à grande échelle d’après les travaux de Jacques Perriault. Voir aussi Moeglin P., 2010 , Les industries éducatives, PUF. Que Sais-Je ?. France.

${ }^{29}$ Barbier JM., 2010, "Cultures d'action et modes partagés d'organisation des construction de sens ", Revue d'anthropologie des connaissances.
} 
dans les valeurs portées par le vocabulaire quotidien comme les outils de valorisation du contrôle de gestion.

\section{Intérêt général, bien commun, service à l'apprenant}

Quelle est la genèse des valeurs ? Dans un premier temps, une valeur est relative à celui qui évalue ${ }^{30}$. Dans un deuxième temps, ces valeurs sont abstraites de l'évaluateur et de la situation pour être considérées comme des absolus. Avant de se demander quelles sont les valeurs du Ced, il faut donc se demander qui sont ceux qui l'évaluent. Nous comprendrons alors leurs critères, leurs logiques, qui génèrent les valeurs de l'institution.

Le Ced est placé sous la double tutelle du Ministère de l'éducation nationale et du Ministère de l'enseignement supérieur et de la recherche. La première autorité tutélaire subventionne le Ced pour qu'il assure le service d'académie en ligne pour les publics ne pouvant pas être scolarisés jusqu'à l'âge légal. La finalité est alors de former tout citoyen français en lui donnant un socle commun de connaissance, sur lequel il sera libre d'ériger sa propre personne et de sculpter sa personnalité selon ses expériences individuelles. C'est une mission d'intérêt général codifiée par la loi (l'article L 111-1 du code de l'éducation fait de l'éducation la «priorité nationale »), rappelée par les élus de notre démocratie (par exemple les accords de Bologne puis de Lisbonne) et mise en œuvre par l'administration (dont le Ced). La valeur d'intérêt général du Ced est ainsi mesurée par des valeurs qui relèvent de l'économie de la connaissance : niveau de qualification d'une classe d'âge, lien formationemploi, compétitivité par rapport à d'autres territoires. L'intérêt général est un intérêt administratif et national. Quand il est international, c'est pour valoriser le territoire et la langue par rapport à ceux des autres nations. Il relève de la partie la plus ad-ministrative du Ced, dépendante du Ministère de l'éducation nationale.

Il en va autrement du deuxième co-tutélaire : le Ministère de l'enseignement supérieur et de la recherche. Avec l'influence anglo-américaine d'éducation supérieure payante et de recherche plus proche des applications économiques privées, la demande faite au Ced est

\footnotetext{
${ }^{30}$ Nous avons soutenu en 2011, à l'université de Paris Ouest Nanterre la Défense, une thèse de doctorat en philosophie (éthique) portant sur la notion de valeur.
} 
d'être à budget équilibré dans les enseignements supérieurs délivrés. La valeur de l'intérêt général national laisse donc sa place à un bien commun : celui de la monnaie. La rentabilité demandée est calculée à plus court terme et chaque formation est supposée dégager une marge positive. Ces valeurs économiques, qui l'apparentent aux industries et commerce privés, sont un bien commun mondial (au sens du «common good» anglais) : celui de la quantification des actions et de leur évaluation sous forme monétaire, avec une visée à plus court terme que celles des États. Nous allons montrer comment les valeurs industrielles et commerciales ont fait irruption dans le quotidien de l'institution avec la réforme des métiers en 2010.

Ces deux tables de valeurs: intérêt général administratif (qualification, emploi, compétitivité nationale) et bien commun industriel et commercial (quantification, monnaie, profit à court terme) se croisent sur un terrain commun et fertile. Le Ced est en effet l'hybridation de ces deux cultures et des valeurs qu'elles portent en germe. Public et privé, administration et industrie commerciale, s'unissent pour former le quotidien des employés. L'institution, qui était simple moyen au service de fins extérieures (les ministères) voit alors fleurir ses propres valeurs - celles de son secteur d'activité. Aux valeurs imposées par les autorités servies s'ajoutent des valeurs endogènes, liées à l'activité de l'institution. Qualité de l'enseignement délivré, autonomie et liberté de l'apprenant du fait de la distance, pertinence de la formation qui, tout au long de la vie, intervient au moment idoine et au format adapté... décrivons ces valeurs en actes avec les données issues de notre observation-participante ${ }^{31}$.

\section{$\underline{\text { L'évolution linguistique }}$}

La culture éducative du Ced a été profondément bouleversée en 2010 avec une réforme qui a remodelé son organisation. Des directions métiers sont apparues : innovation, offre et services, commerciale, scolarité, production ; direction métiers qui devaient collaborer quel que soit l'un des huit sites géographique d'implantation du Ced.

Cette réorganisation a été l'occasion d'une violence symbolique consistant à renommer plusieurs métiers de la tradition administrative en les rapprochant des appellations

\footnotetext{
${ }^{31}$ Nous avons occupé pendant 3 ans un poste de Responsable de Formation - Chargé d'Ingénierie de Formation, en charge de formations sur les sites de Vanves et Vinci (Poitiers).
} 
du secteur industriel et commercial ${ }^{32}$. Ainsi, à la place des pôles et services administratifs, des lignes de marchés ont été crées et regroupées en unités d'affaires. Au plus haut niveau, le recteur a renoncé à ce titre de l'administration éducative pour se désigner comme directeur général, plus proche des appellations du privé ; le Ced étant ainsi dirigé et non plus régi ${ }^{33}$.

Le travail des chargés d'ingénierie de formation a été remodelé par la mise en place d'un processus du cycle de vie de l'offre. Chaque produit de formation est décrit les étapes de son cycle de vie : création, lancement, développement, suppression - selon le cadre marketing tel qu'il a été construit dans les États-Unis d'après guerre et tel qu'il s'enseigne dans les grandes écoles de commerce françaises où recrute en partie le Ced.

Une indécision de terminologie porte sur la «cible » visée : tantôt client (un service client est là pour répondre aux demandes; la direction commerciale mène des actions pour fidéliser la clientèle), tantôt inscrit (pour la scolarité qui enregistre les dossiers et fournit les relevés de notes et attestation de fin de formation), tantôt apprenant (pour les responsables de formation qui personnifient la pédagogie ou les chercheurs de la direction de l'innovation qui améliorent la qualité technique des modes d'apprentissage).

Ce glissement sémantique du vocable de l'administration éducative vers des appellations du secteur industriel et commercial est toutefois limité. En effet, alors que le secteur privé français, dont celui de la formation et de ses nombreux organismes, est sensible aux discours anglo-saxons («blended learning», «marketing », « benchmark»), l'établissement public suit les circulaires ministérielles sur l'emploi du français : on y parle ainsi de formation hybride, de mercatique (dans certaines formations) et d'étalonnage et d'étude de marché. Les exemples cités relèvent de la simple traduction, celle-ci n'est pourtant pas toujours évidente, comme dans le cas de la formation ouverte (le Ced dispose d'une école de l'ingénierie de la formation distance - Eifad) qui désigne en France une formation qui laisse ouvertes les portes de la classe et se fait donc à distance ; alors que, dans le monde anglophone, marqué par l'Open University anglaise et ses épigones indiens ou chinois, l'open education désigne une formation ouverte sans prérequis de diplôme.

\footnotetext{
${ }^{32} \mathrm{~A} \mathrm{l}$ 'heure de la relecture de cet article, ce mouvement est atténué par un retour en arrière vers la tradition administrative et son vocable, ressac qui ne remet pas en cause le mouvement d'ensemble.

${ }^{33} \mathrm{Ce}$ qui marque un changement de la culture de l'honneur à la française qui ploie sous le poids des cultures mondiales, selon l'expression de Philippe d'Iribarne.
} 
Poursuivant notre analyse du vocabulaire et des façons de dire, nous constatons que certains non-dits ${ }^{34}$ sont très parlants. Ainsi l'argent fait partie de ces réalités mises en valeur à force de vouloir les taire. Nous avons observé la vie quotidienne du Ced sur deux sites très distincts : le site de Vanves, ancienne lieu de direction, est resté très «vieille école » avec une population excessivement diplômée (le site est spécialisé dans les formations du supérieur) et possédant un capital intellectuel excédant leur capital monétaire. Les locaux sont en grande partie anciens : escaliers dont la peinture rappelle les couloirs de nos plus vieilles universités, moquette usée par les ans,... alors même que le site est de ceux qui reviennent le plus cher à l'institution car il est en région parisienne. À l'opposé, la direction générale nouvelle est placée sur le site du Futuroscope de Poitiers, distante de tout (sauf de Paris puisqu'une ligne de TGV dessert la gare du parc) est résolument moderne et orientée vers les techniques de l'image. L'architecture aux vitres teintées et à l'ambiance aseptisée rappelle les sièges sociaux des entreprises modernes de la Défense, ou encore des celui des cabinets de conseil.

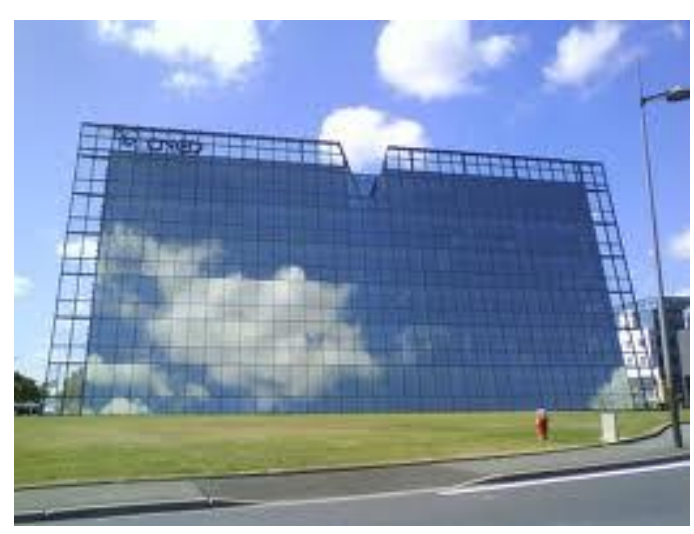

$\underline{\text { Site de la direction, près du Futuroscope }}$

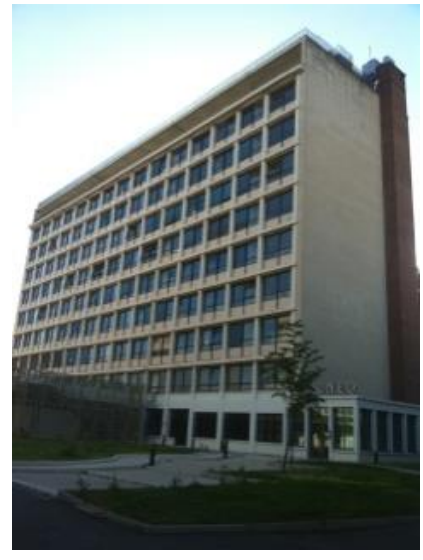

$\underline{\text { Site de l'ancienne direction, Vanves }}$

\section{Vues du CED}

À Vanves, même parmi le personnel administratif de catégorie A, la tenue vestimentaire est décontractée et la hiérarchie informelle des responsables de formation est basée sur une sacralisation des parcours scolaires. Certaines disciplines valent plus que d'autres (car le concours d'agrégation est plus couru), l'adjectif « normal » revient assez souvent pour désigner ce que l'on fait et le couloir est placé sous l'autorité d'un ancien élève

\footnotetext{
${ }^{34}$ Nous faisons référence aux travaux du sociologue Pierre Bourdieu pour ces analyses sur ce que parler veut dire et sur les violences symboliques dans un champ scientifique.
} 
d'une université américaine (Harvard). Quand les titres scolaires ne suffisent pas, ou pour compenser quand le parcours est autre, c'est la course au partenariat renommé avec telle université ou telle maison d'édition en vue. Et dans ce cas on ne parle pas d'argent mais de prestige. Le silence sur la monnaie est parfois rompu par un cri : «fric » - ce dont on dit toujours manquer mais sans lequel on affirme pouvoir très bien faire.

À l'opposé, dans les couloirs de la direction commerciale, située au sein même du siège du Ced, les «benchmark » se multiplient et les offres promotionnelles sont proposées dans nombre de supports commerciaux pour appâter le chaland. On croise souvent des employés costumés et cravatés, l'expression «-20\% pour une double inscription » est valorisante sur un document commercial et on compte en euros le prix de conquête d'un nouvel inscrit, avec entrain et réalisme. Plusieurs employés sont issus de grandes écoles de commerce et ont donc été formés à ces logiques marchandes et à un esprit capitaliste débarqué d'outre-océan avec la guerre économique.

Certes, cette description ethnologique ${ }^{35}$ opposant deux sites est réductrice. Montrer c'est cacher, car l'index qui pointe vers telle partie de la réalité délaisse le reste. Pour saisir la réalité sociale à pleines mains, il faudrait dire qu'à Vanves plusieurs employés ont été recrutés après des écoles de commerce pour aller dans le sens actuel de l'évolution de l'institution, de l'administration vers l'industrie et le commerce. D'autres sont purement administratifs et ont fait leur carrière dans d'autres organisations éducatives publiques. Et, bien sûr, il faudrait pouvoir comparer avec les six autres sites géographiques. Mais ils ne sont pas à portée de main de notre étude : nous avions sous les yeux un site quotidiennement et l'autre site mensuellement. À cette limite d'extraction de données de données de terrain, il faudrait rajouter enfin nos lunettes scientifiques issues des sciences sociales et de l'éducation. Comme pour le hors-champ des cinéastes, plusieurs éléments de réalité ne rentraient pas dans notre champ scientifique et n'ont pas retenu notre regard.

\section{$\underline{\text { La révolution numérique }}$}

Après l'évolution linguistique qu'a introduite la réforme de 2010, décrivons un autre changement qui traduit le passage de la culture administrative à la culture industrielle et

\footnotetext{
${ }^{35}$ Dans la lignée des travaux des sociologues Erwin Goffman sur les cadres de l'expérience sociale et Howard Becker sur les acteurs sociaux et leurs interactions.
} 
commerciale. Il s'agit d'un projet, en cours en 2012, consistant à instaurer un outil de contrôle de gestion de calcul des coûts sous forme de tableur et qui représente une véritable révolution numérique pour les responsables de formation. En effet, c'est un parachèvement de la logique industrielle de l'organisation scientifique du travail : des outils sont donnés aux cadres pour qu'ils puissent eux-mêmes calculer et optimiser leurs activités.

Dans le sillage des travaux sur la mesure de François Vatin ${ }^{36}$ et de la sociologie économique telle qu'elle s'enseigne à l'École normale supérieure, nous voulons montrer que ce calcul des coûts aidé par les outils du contrôle de gestion est un construit social. La création du contrôle de gestion, rattaché à la direction générale, est récente. Elle a fait l'objet du recrutement d'un employé issu du monde industriel privé. Celui-ci a été chargé de collecter les modes de calcul des coûts des différents métiers et sites du Ced avant de les intégrer tous dans un tableau tenant sur une feuille de calcul du logiciel Excel. Cet outil a ensuite été autorisé par la direction générale qui en a imposé l'utilisation à tous les responsables de formation, afin de cadrer leur activité de cadre en les obligeant à valoriser leurs projets de formation : recettes escomptées mises en balance avec les dépenses engagées. Si le dispositif de mesure de la valeur a été critiqué par les employés une fois mis en place, force est de constater qu'ils ont participé à la construction de ce mode de calcul qui leur a ensuite été imposé.

Quelles furent les principales critiques ? Les points de mésententes étaient la difficulté à parler un langage commun entre les différents métiers et à trouver des unités de mesure stables. Une fois le tableau créé, il a fallu rentrer dans les cases du tableur et ceci est apparu compliqué : qu'est-ce qu'un «produit multimédia »? Est-on sûr que l'estimation des coûts que le responsable de formation donne est la même que celle de la responsable de la production? Lorsque l'un appelle l'autre pour vérifier, il apparaît que ce qui est demandé en coûts journée-homme d'un chef de projet multimédia dans le tableau du contrôle de gestion se mesure dans l'autre direction comme un coût à la page de site Internet... L'outil de calcul de la valeur pose des problèmes de qualification de la réalité tout autant que de quantification des coûts. Apprendre à compter la valeur d'une formation en euros avec cet outil de pilotage a constitué un changement culturel profond.

\footnotetext{
${ }^{36}$ Par exemple Vatin F. (dir.), 2009, Evaluer et valoriser. Une sociologie économique de la mesure, PUM, Toulouse. On retiendra notamment la présentation de la thèse d'Alexandra Bidet concernant la métrologie et la construction sociale des normes de calcul. Cette approche historique des calculs peut être mise en perspective avec celle d'Olivier Godechot qui, dans sa sociologie des marchés financiers, rattache l'utilisation de telle ou telle méthode de calcul à l'origine sociale des traders.
} 
Ce qui est certain c'est que l'outil d'aide à la gestion objective les coûts. Au lieu que chacun ait une vision subjective, soumise à des données éparses, un objet est construit, identique pour tous, qui rassemble toutes les données et permet de prendre de la hauteur face à elles. La décision est alors facilitée, la gestion est aidée plus que contrôlée. Cet objet comptable est d'ailleurs une véritable machine à calculer : une fois que les responsables de formation acceptent de «rentrer dans les cases» en remplissant le tableau, ils voient apparaître automatiquement une image de la rentabilité espérée sur les premières années du projet. Plus qu'un tableau comptable, l'objet implique l'assimilation d'un nouveau paradigme : celui du tableur, de la raison informatique.

Le formatage des activités des responsables de formation par le contrôle de gestion a en effet donné lieu à une formation aux outils informatiques. Pour se servir du tableau de calcul des coûts il a fallu se former au tableau et au logiciel-même permettant l'existence du tableau. Cette révolution ne va pas sans rappeler celle que Jack Goody ${ }^{37}$ a appelé la révolution de la raison graphique. Comme l'apparition de l'écriture a transformé les façons de penser, l'utilisation de l'écriture informatique change profondément la façon de penser des responsables de formation. Certes le tableau existait avant : croiser une ligne et une colonne dans une cellule n'est pas une opération mentale nouvelle pour les responsables de formation qui l'ont déjà appris sur du papier. Mais que les cellules soient liées entre elles et que l'une s'additionne à l'autre pour instantanément donner la somme dans une tierce cellule, ceci est révolutionnaire. Le dynamisme de l'écriture numérique est la principale nouveauté. Elle se retrouve dans les graphiques - là aussi opération connue des responsables de formation - qui se mettent à jour instantanément. La courbe de rentabilité change de tracé lorsque l'on fait varier la quantité de fascicules de cours à imprimer. Emerveillés devant l'outil, l'ancienne génération des responsables de formation apprend ce que les plus jeunes connaissent depuis le lycée.

En-deçà même de la logique du logiciel, c'est la raison informatique qui s'impose. La décomposition des informations en unités très fines qui se recomposent en langages qui s'enchâssent (du binaire fondateur à l'interface du tableur décrit) permet à l'information d'être retraitée et valorisée à différents niveaux. Et cette raison informatique formate les pratiques de formation : elle permet de penser en unité de formation fines que l'on recompose selon celui que l'on veut former (ce dont il a besoin et dans quel ordre) et donne les moyens

\footnotetext{
${ }^{37}$ Goody J., 1979, La raison graphique. Éditions de Minuit. Le sens commun. France.
} 
techniques de le faire. Les unités d'enseignement sont en effet réparties en modules qui, en quelques clics, peuvent être agencés pour construire une formation adaptée.

\section{Conclusion}

Comprendre la trajectoire du Ced, de l'administratif vers l'industriel et commercial, peut déboucher sur une physique sociale, selon le mot d'Auguste Comte ${ }^{38}$. Celle-ci permet de voir l'institution comme un corps mobile, corps doté d'une inertie et sur lequel s'appliquent des forces. L'inertie est composée de traditions sociales (de recrutement ou de hiérarchie valorisant les diplômes, de certains tabous quant à l'argent) et d'habitudes (des façons de compter, un vocabulaire et les valeurs qu'il implique). Cette inertie est d'autant plus importante que l'institution est grande («c'est partout pareil ») et vieille (« on a toujours fait comme ça »). Les forces qui s'appliquent sur l'institution mobile seraient l'attraction pour les savoirs que l'on prononce avec gravité, les technologies de la communication qui électrisent le marché de l'enseignement et enfin la puissance atomique de la fusion en une seule cotutelle des deux ministères. Ainsi les mouvements du Ced seraient suscités par des forces plurielles et freinés par une inertie complexe.

C'est ce solide humain que nous avons voulu décrire: les formes profondes qui se forment avec le temps et font ainsi l'identité de l'institution sous ses transformations superficielles. Habits valorisés, habitats de travail, habitudes de langue: les hommes collaborent en une réalité sociale assez dure pour durer et mobilisée par des forces plurielles.

La physique sociale est une hypothèse fondatrice de la sociologie. Ce qui est certain c'est que la vaste institution du Ced connaît une division du travail poussée qui n'est pas sans rappeler la manufacture d'épingles d'Adam Smith. Privée ou publique - ce n'est jamais qu'un changement de droit applicable - la réalité sociale est celle d'une usine à savoirs, digne de celles de l'âge industriel. Sous l'éventuel passage d'un statut public à un statut privé, passage qui se ferait brutalement sur le papier, un lent glissement culturel s'opère : celui des hommes et de leurs compétences.

\footnotetext{
${ }^{38}$ Créateur français du mot « sociologie » et ayant hésité avec l'appellation « physique sociale ».
} 


\section{L'entrée « management » dans l'enseignement : comptabilité, mercatique, gestion de projet}

Notre tableau institutionnel présente une organisation qui évolue, du fait du contexte international relayé par les pressions ministérielles, vers une logique marchande. Les formations à distance du supérieur sont vendues et il faut donc un personnel adapté à ces nouvelles missions. Ainsi nous entrons dans notre étude par cette nouvelle gestion publique (New Public Management), aujourd'hui dépassée, et, travaillant comme cadre, une partie de nos modes de pensée sont déterminés par les sciences de gestion.

Les quatre textes suivants proposent une analyse de ces outils en action au quotidien dans l'institution. Nous faisons un effort anthropologique pour réfléchir les sciences de gestion dans un projet plus général de sciences humaines de l'éducation. Ainsi nous allons présenter successivement le calcul de la valeur d'une formation par une comptabilité et une finance adaptées - ce qui ne va pas sans des conséquences économiques et une centration sur la demande du marché. Puis nous verrons comment se construit l'image de marque de l'institution et de ses modes d'apprentissages par une mercatique orientée sur le numérique et sur l'originalité des parcours de formation atypiques et leur imaginarie (sport de haut niveau, vie itinérante de voyageur ou d'artiste, etc.). Enfin, les deux derniers textes autour de projets d'ingénierie de formation déconstruisent les techniques de gestion de projet en s'intéressant aux notions temporelles qu'elles mobilisent. Le temps linéaire et orienté de l'action finalisée, reconstruisant à un instant «i » un passé explicatif et futur probable, est déconstruit à la faveur d'un temps cyclique où reviennent des «moments forts » collectifs. Les différents projets s'encastrent dans des temporalités allant croissant selon le niveau hiérarchique dans l'organisation et la portée des décisions.

Plus précisément, le premier chapitre de cette section discute les apports des sciences de gestion aux sciences de l'éducation, en prenant le cas des outils utilisés par les ingénieurs de formation lors de leur travail de conception. Ainsi l'étude de marché, le cycle de vie de l'offre ou encore l'image de marque sont travaillés en amont puis une comptabilité analytique permet de mesurer la valeur anticipée et financière d'un produit de formation. Cet usage des techniques de gestion en éducation est à modérer, d'une part du fait des limites et impulsions juridiques de la puissance publique qui légifère, mais aussi parce que les valeurs éducatives de qualité ou encore de pertinence pédagogique heurtent parfois frontalement une logique 
purement marchande. Nous sommes conscients, de par nos origines familiales dans le milieu éducatif, que les sciences de l'éducation se heurtent parfois aux logiques gestionnaires. Ceci par conflit de section universitaire mais aussi, plus communément, par déontologie de métier et orientation politique fondamentale. Nous nous efforçons donc de tenir un discours qui ne fera pas l'objet de critiques et de suites virulentes, oubliant les débats de fonds pour ne faire entendre que l'erronée « droitisation » de savoirs un temps légitime.

Cette logique marchande est hypostasiée dans le second chapitre traitant de l'imaginaire vendeur des formations. En effet, il s'agit de montrer la carte commeciale de l'institution, dont le slogan était «connecté à votre avenir », et qui met en avant des parcours atypiques de formation sur son site Internet pour attirer de nouveaux inscrits. Ainsi les vies de voyageurs itinérants (sportif de haut niveau, artiste, tour du monde en famille à la voile, etc.) sont annoncées comme possibles grâce à l'institution et ses formations à distance qu'elle met au service de projets personnels. Le webzine étudié, outil de marketing de la formation, permet de tracer une topographie de cette imaginaire et de ces récits merveilleux en les regroupant sous deux archétypes de récits de formation vendeurs : l'exploit individuel et l'aventure familiale. À chaque fois des éléments oniriques issus du loisir (sport, culture, voyages) sont associés aux études à distance pour inciter les internautes à s'inscrire dans les dispositifs de formation. La carte de ces imaginaires-là est donc aussi une carte commerciale et tous ses chemins convergent vers l'inscription administrative dans les programmes de formation.

Enfin les deux chapitres suivants traitent, non plus du calcul comptable de valeur ou de la valorisation commerciale d'une formation, mais des techniques de gestion de projet lors de sa conception - techniques relevant elles-aussi des sciences de gestion. Nous présentons ainsi d'abord un projet d'ingénierie de formation (le «projet $G$ ») qui consiste à développer une école de gestion en ligne, d'une durée de trois années, visant un public de néo-bacheliers, avec une équipe pédagogique d'enseignants. Décrire ce projet nous permet de présenter l'organisation et l'ingénierie de formation en instrumentant les sciences de la gestion en sciences de l'éducation. Nous montrons ainsi comment la gestion passe par des gestes efficaces d'actants partageant une culture d'action commune, c'est-à-dire des procédures et des formes institutionnelles. Notre ethnographie se centre sur l'action de conception telle qu'elle se matérialise par des formes et formulaires particuliers et cherche à définir les modalités temporelles de cette matérialisation. Ce qui est repris et développé dans le quatrième chapitre : l'ingénierie de formation est décrite comme une activité de conception marquée par des temps forts qui reviennent régulièrement et rythment l'activité. Un modèle graphique en 
est présenté et ouvre ensuite sur une analyse de la mise en récit de la formation par ses concepteurs-mêmes. Le temps du projet de formation n'est pas celui de son récit, toujours orienté par des intérêts autres. Le temps, ressource cadre des techniques de gestion de projet mais rarement réfléchi comme tel, est ainsi déconstruit empiriquement pour être repensé anthropologiquement tel qu'il se présente dans les actes du quotidien. La vision linéaire et finalisée (la flèche du temps) est remplacée par une vision cyclique, scandée de moments répétitifs et d'intensités variables.

Nous pouvons finalement questionner ce modèle anthropologique de réflexion des sciences de gestion en actes dans l'institution. En effet, dans Le projet Inter Lang : analyse d'un groupe de travail en grande école ${ }^{39}$, nous avions fait œuvre d'ethnographe en montrant l'acquisition de compétences de gestion dans une formation. Le présent ouvrage peut en être vu comme la suite chronologique. Nous étudions en effet ici le lien formation-emploi, non pas sur le marché du travail où est valorisé la formation, mais dans l'utilisation de l'outillage formel pendant l'emploi. À cet égard, la formation de gestion que nous avons eue, par l'étude menée au préalable, influence les analyses anthropologiques : le regard sur l'institution et sur l'emploi éducatif est préformaté. De même il est à préciser que nous étions à un poste d'observation mobilisant les sciences de gestion (nous étions responsables de formations en gestion). Une anthropologie menée au regard d'un poste d'observation comme responsable de formations en mathématiques, formé aux mathématiques, aurait eu des perspectives différentes ${ }^{40}$.

\footnotetext{
${ }^{39} \mathrm{https}: / /$ halshs.archives-ouvertes.fr/halshs- 01233788 . Ce document en ligne est un carnet de terrain ethnograhique d'études menées en grande école en parallèle d'un parcours doctoral en sciences sociales.

40 Une discussion peut être entamée pour préfigurer notre conclusion sur l'anthropologie épistémique. En effet, dans le lien formation-emploi que nous étudions, nous pensons que le critère d'emprise d'une formation sur le réel, ou le pouvoir d'action de cette connaissance, ne sont pas valables pour hiérarchiser les formations. Nous montrerons en effet comment la perception du réel (et donc de l'emprise sur celui-ci) dépend des conceptions apprises en formation. Ainsi l'on vit dans le monde auquel on a été formé et vouloir mesurer le pouvoir de ses connaissances à l'aune de notre perception de leurs effets sur le monde paraît tautologique.

La hiérarchie des connaissances et des formations semble le fruit indéterminé de l'histoire des rapports de force entre pays (comme pour la nouvelle gestion publique importée des États-Unis) et institutions (en France, les grands établissements et grandes écoles qui se partagent l'espace de transmission spécialisé et y placent leurs anciens étudiants formatés).
} 


\section{$\underline{\text { Introduction : instrumenter les sciences de gestion }}$}

Concevoir une formation pour adultes dans un organisme public d'enseignement à distance pose des questions de gestion, que ce soit au responsable de la formation s'y ingéniant - formé ou non à cet exercice au préalable - ou à l'observateur attentif effectuant un travail de recherche de type ethnographique en milieu éducatif (Marty, 2015). Et l'on sait que le praxéologue est 'participantobservateur' plutôt qu'observateur participant scientifique, les deux perspectives peuvent être réunies en la même personne sur le terrain. Comment concevoir une formation pour adulte de valeur ? C'est-àdire, rentable économiquement ? Comment, alors, mesurer cette valeur et quels outils employer pour la maximiser?

Nos précédents travaux de sociologie par observation participante ont montré comment une telle logique gestionnaire rencontrait des résistances de la part d'acteurs (surtout dans le secteur public : Marty, 2014) revendiquant des valeurs dépassant l'argent, l'économique et touchant à leur identité professionnelle (service public, service à l'apprenant, qualité pédagogique, refus du chiffre réducteur par les littéraires, etc.). Nous voulons ici revenir sur cette enquête en allant « dans le sens de l'histoire » (pour reprendre les directives des organismes de formation étudiés, largement d'inspiration du public management néolibéral) pour exposer par le détail le calcul économique de la valeur d'une formation par les outils de gestion utilisés sur le terrain et ne laisser que la part congrue à la critique qui faisait l'objet de nos précédents travaux.

Notre problématique est donc complexe: nous proposons un document de sciences de l'éducation qui fait état des théories des sciences de gestion mises en pratique en France, pour un lectorat de sciences de l'éducation. Nous ne cherchons donc pas à élaborer de nouveaux outils de sciences de gestion propres à l'éducation, mais à présenter ceux qui sont déjà présents dans la réalité sociale des institutions éducatives étudiées. À la différence d'un travail de sciences de gestion appliqué à l'éducation voulant innover par son terrain d'application ou ses instruments d'analyse, nous voulons donner à la communauté de recherche en sciences de l'éducation (non nécessairement tous économistes ou gestionnaires) un état fidèle, synthétique et actualisé, des savoirs fondamentaux utilisés en formation des adultes. Car, de fait, sur le terrain, un avantage considérable est donné aux acteurs maîtrisant ces savoirs (parce qu'ils y ont été formés au lieu de les apprendre tardivement sur leur poste de travail) par rapport à ceux pénalisés par leur ignorance de l'approche gestionnaire. Il s'agit donc 
bien d'une recherche scientifique, relevant de l'éducation et non de la gestion, basée sur des données de terrain et, pour aller au-delà d'une simple praxéologie, cadrée par un corpus théorique. La difficulté problématique consistant à faire dialoguer deux cultures disciplinaires différentes, autour de réalités sociales en milieu éducatif.

Notre hypothèse centrale est qu'une enquête de terrain en sciences sociales permet de connaître les modèles gestionnaires mis en pratique et d'en connaître l'état et les détournements actuels. Questionner la notion de valeur devrait ainsi nous permettre de voir les logiques gestionnaires en pratique dans les milieux éducatifs et d'en rendre compte fidèlement. Cette hypothèse, si confirmée, devrait nous permettre de continuer, en fin de document, à développer la thèse du lien entre évaluation de la formation et valeurs de référence.

Le terrain ethnographique, dans une «industrie éducative » (Moeglin, 2010) de premier plan pour l'enseignement à distance public français était situé à un poste d'observation de cadre intermédiaire. En tant que responsable de formation chargé de l'ingénierie de formation, aux côtés de quatre vingt autres titulaires de la même fonction répartis en huit site géographiques ayant chacun leur spécialité en France, nous avons observé les pratiques de gestion s'immiscer dans le secteur éducatif public. Nous n'étions pas au cœur des débats de la direction générale, en partie relayés par la revue scientifique éditée par l'administration, mais pouvions voir comment un personnel relativement âgé (une quarantaine d'années en moyenne) et non nécessairement sensible aux discours gestionnaires, devaient se plier à cette logique par le biais de formulaires administratifs et ordres hiérarchiques édictés dans la langue des sciences de la gestion.

Nous étions, quant à nous, sensibilisé à la gestion pour y avoir été formé dans une grande école, en parallèle d'un premier travail de thèse de sociologie où nous avions tenté de mettre à distance les savoirs enseignés. C'est donc là qu'il faut trouver l'origine de notre questionnement sur les rapports des agents éducatifs à la discipline des sciences de la gestion et à ses pratiques. Nous nous centrerons sur la question de la valeur car elle était saillante dans les débats sur le terrain (c'était le " problème » du calcul de la valeur économique) et qu'elle était charnière dans notre seconde thèse de philosophie (traitant des valeurs d'une communauté professionnelle) ${ }^{41}$.

41 Les données concernant nos deux types d'écrits doctoraux sont disponibles sur https://cv.archives-ouvertes.fr/marty 


\section{$\underline{\text { La mercatique ou la valeur machande }}$}

\section{Valeur pour le client ou valeur pour le consommateur}

Le premier outil de gestion employé par les cadres intermédiaires de la formation des adultes en organisme public est la mercatique (ou marketing pour reprendre le terme anglais plus couramment prononcé sur le terrain). Les concepteurs de formation s'ingénient à trouver des clients (quoique la pudeur pédagogique du langage dans le secteur public amène parfois aussi à s'exprimer en termes de « usagers» ou «apprenants ») auxquels vendre leur formation. Dans les organismes privés la distinction entre le client et le consommateur se calque sur celle entre le responsable de formation d'une organisation privée souhaitant mettre à son catalogue de formations les productions de l'organisme (c'est le client de l'organisme de formation) et l'employé de l'entreprise qui assistera à la formation (c'est le consommateur). Ainsi la valeur de la formation n'est pas toujours la même selon qu'elle est calculée par le client (ce qu'il paie en anticipant la valeur ajoutée pour les forces productives de son organisation) et pour le consommateur (le crédit de temps passé au sein de son organisation pour une formation qu'il valorisera au sein de cette dernière mais peut être aussi en dehors).

\section{Une conception de la formation avec les outils de mercatique}

\section{Études de marché}

Le concepteur de la formation effectue une veille permanente sur ses marchés et lit l'ensemble des indicateurs (presse professionnelle, actualité juridique,...) pour trouver une demande de formation à laquelle il pourrait répondre par une offre. Il construit alors un produit à offrir ciblant la demande. Le produit ainsi construit dans une logique de mercatique sera mis sur le marché par des canaux de communication: catalogue de formation papier, méls publicitaires, relances ciblées et rencontres de clients importants par l'équipe commerciale de l'organisme.

Quand ces opérations de conception mercatique sont formalisées, comme dans l'industrie éducative constituant notre terrain, des formulaires administratifs de conception ( sont à remplir en indiquant la quantité de clients potentiels mais aussi leur qualité, c'est-à-dire des éléments pour mieux cerner le prix qu'ils seraient prêts à payer (selon leur âge, leur catégorie socioprofessionnelle, etc.), le tout pour déterminer un chiffre d'affaire potentiel de la formation proposée. 
Le concept mercatique de cycle de vie de l'offre, relativement récent dans cette discipline, permet d'avoir une idée de l'espérance de vie du produit de formation à concevoir et des recettes espérées à chaque étape de son développement : le chiffre d'affaires lors de son lancement, puis de sa croissance, de son arrivée à maturité et enfin celui de sa période de déclin. Pour des raisons économiques évidentes, les organismes préconisaient à ses concepteurs de travailler à des produits de formation pérennes, susceptibles d'être maintenus sur le marché pendant une longue période de temps et constituer des vaches à lait (selon le vocable imagé du Boston Consulting Group et des matrices que cette institution a élaboré, qui s'enseigne en gestion et fait partie du langage de terrain). Les promotions s'inscrivant à la formation s'accumulent alors et le gain financier espéré ne cesse d'augmenter - ce qui penche en faveur d'un investissement dans la conception de ce produit plutôt que d'un autre.

L'utilisation des «4P»du marketing est fréquente sur le terrain : le Prix, la Promotion, le Produit et la Place de vente sont demandés aux chefs de projets ou ingénieur de formation qui doivent penser au marché et aux débouchés de ce qu'ils ont à concevoir. Cette vision quadrangulaire est un cadre de pensée qui détermine les discours des acteurs. Le Produit, notamment, est pensé en termes de marque sur laquelle il faut communiquer pour en augmenter la valeur. Ainsi les clients vont acheter un titre de formation (souvent développé en objectifs pédagogiques montrant les compétences à acquérir sous forme de verbes d'action et qui reviennent à assimiler voir s'assimiler au titre en l'incorporant), dans le catalogue de tel organisme dont le prestige importe (notamment par ses liens avec la branche professionnelle et sa reconnaissance par les OPCA, mais aussi de par ses méthodes pédagogiques propres) et avec tel ou tel formateur qui est reconnu pour son expérience ou ses publications sur le sujet. Le produit mercatique peut ainsi se décomposer en différentes marques hiérarchisées qui en augmentent la valeur sur le marché, aux yeux des clients regardant la plaquette commerciale de présentation ou écoutant le discours du vendeur.

\section{Limites juridiques d'une mercatique de la formation}

Une telle approche mercatique est cependant limitée dans le sens où le marché qu'elle met en avant et qui s'explore par des études de marché est parfois réifié, plus encore que régulé, par le droit. Certes l'actualité juridique peut susciter un nouveau besoin de formation (connaitre une nouvelle réglementation par exemple) mais le droit est surtout un cadre (par exemple la loi de 1971 sur la formation professionnelle continue et les différentes réformes qui l'actualisent), voire une prescription 
à laquelle il faut répondre. Plus loin encore, l'organisme public de formation à distance que nous avons étudié se détache souvent de considérations économiques de type mercatiques pour répondre à un acte unilatéral de la puissance publique, à savoir le ministère qui le subventionne et qui impose de créer un vaste dispositif (parfois estimé non rentable à court terme) sur tel ou tel domaine. La mercatique entretien ainsi des relations complexes avec le droit et la valeur marchande cède parfois la priorité aux décrets et législations.

\section{$\underline{\text { La comptabilité analytique et la valeur de fabrication }}$}

Les concepteurs de formation étudiés, dans le deuxième terrain en industrie éducative de la formation à distance publique, où une comptabilité analytique se mettait peu à peu en place, procèdent par comptabilisation des coûts pour estimer la faisabilité de leur projet de formation. Ils calculent ainsi la valeur de fabrication ou valeur industrielle. Ils ont ainsi des tableaux électroniques à compléter pour mesurer les dépenses liées à tout projet de formation. L'exercice qui leur est demandé est de remplir les cases d'un tableur le plus « sincèrement » possible, en intégrant des coûts dont ils sont informés par différents services qu'ils centralisent (scolarité qui inscrit, production qui imprime et fabrique les sites Internet, partenaires extérieurs comme des universités ou des enseignants du second degré qui rédigent les documents pédagogiques, etc.).

L'élaboration de ces tableaux est un processus complexe qui amène plusieurs services à coopérer pour trouver une unité de mesure commune (comptabiliser des pièces produites ? des jours/hommes ? avec quel degré de finesse ?) et un langage commun (un même « document » devient un «fascicule » voire un « cours » selon les différentes étapes de la chaîne opératoire de fabrication qui le fait passer dans différents services). Et une fois le tableau construit, reste encore à l'utiliser - et nous avons constaté des instrumentations de cet outil de gestion pour servir des fins individuelles ou de service. La « sincérité » demandée par le contrôle de gestion était donc, comme nous allons le montrer, mise à l'épreuve des faits et des ambitions.

\section{Evaluer les coûts}

Nous présentons ci-dessous un tableau similaire à celui imposé dans l'institution d'enseignement à distance que nous avons étudiée. Les concepteurs de formation s'ingéniant à créer de nouveaux dispositifs devaient en présenter un bilan comptable sous forme de tableur. Le tableau 
comptait trois feuille de calculs : la première concernait la valeur mercatique (chiffre d'affaires annuel, espérance de vie, selon le prix et le nombre de clients estimés), la seconde la valeur comptable des dépenses (tableau que nous présentons dessous distinguant les dépenses d'investissement de celles d'exploitation), le troisième fait l'objet du sous-chapitre suivant et met les recettes en regard des dépenses dans une analyse financière (seuils de rentabilité, taux de marge, amortissement, etc.).

La deuxième opération de mesure de la valeur concerne ainsi les coûts. Après avoir été attiré par un «marché porteur », le concepteur vérifie que la formation qu'il veut mettre en place aura des coûts inférieurs aux recettes espérées. Ainsi il chiffre les différents postes de dépenses listées dans le tableau ci-dessous :

\begin{tabular}{|c|c|}
\hline Investissement & Coût amortissable durant la période d'exploitation \\
\hline & Journée/homme d'ingénierie de formation \\
\hline & Journée/homme de coordination de projet \\
\hline & $\begin{array}{l}\text { Rédaction des contenus de formation (cours et } \\
\text { devoirs) : achat externe }\end{array}$ \\
\hline & $\begin{array}{l}\text { Mise en forme des contenus de formation par le } \\
\text { service de production }\end{array}$ \\
\hline & Fabrication du site Web par le service de production \\
\hline $\begin{array}{l}\text { Réinvestissement dans } \\
\text { une « réingénierie » }\end{array}$ & $\begin{array}{l}\text { Mise à jour des contenus pédagogiques : nouveaux } \\
\text { programmes, nouveaux devoirs, etc. : achat externe }\end{array}$ \\
\hline Exploitation & $\begin{array}{l}\text { Coût variable selon le nombre de clients et répété à } \\
\text { chaque année du dispositif }\end{array}$ \\
\hline & $\begin{array}{l}\text { Journée/homme de coordination de dispositif de } \\
\text { formation }\end{array}$ \\
\hline & $\begin{array}{l}\text { Communication et vente de la formation par le } \\
\text { service commercial }\end{array}$ \\
\hline & Impression des documents par le service de \\
\hline
\end{tabular}




\begin{tabular}{|l|l|}
\hline & production \\
\hline & $\begin{array}{l}\text { Envoi des documents par courrier papier ou } \\
\text { électronique }\end{array}$ \\
\hline & Correction des devoirs par des prestataires externes \\
\hline & $\begin{array}{l}\text { Inscription de participants par le service de scolarité } \\
\text { présence par des vacataires }\end{array}$ \\
\hline & $\begin{array}{l}\text { Location de salle d'examen et surveillance } \\
\text { Maintenance des sites Internet par le service de } \\
\text { production }\end{array}$ \\
\hline
\end{tabular}

\section{Liste des coûts d'une formation à distance}

Le tableau a le mérite de présenter à chacun des concepteurs de formation la liste exhaustive des postes de dépenses, avec leur unité de mesure (non reproduite ici car trop dépendante de l'institution observée pour être généralisable et d'intérêt scientifique). L'agent public a alors à estimer chaque coût et le tableau en calcule la somme, en la démultipliant selon le nombre d'années d'exploitation du dispositif. En haut de tableau figurent les coûts d'investissement : les journées passées à la conception du dispositif, la mise en œuvre du projet, la commande des cours, leur mise en formation, la production du site Internet, la rédaction des documents administratifs, etc. Puis, pour chaque année d'exploitation du dispositif de formation, en bas de tableau figurent : l'impression des cours, la distribution postale, les frais de commercialisation du dispositif (plaquettes, méls, catalogues, etc.), la correction des devoirs, l'animation des espaces de formation, la location de salle d'examen et le maintien des sites Internet. Ne figurent pas dans ce tableau les frais de structure, somme des coûts des services supports (ressources humaines, service juridique, direction de l'innovation, etc.) que le concepteur, via le tableau qu'on lui donne, réparti sur tout projet de formation et, dans cette institutionlà, estimés à $15 \%$ des dépenses. 


\section{De l'investissement à l'exploitation}

Le tableau présenté se divise ainsi entre, d'une part, le haut de tableau dédié à l'investissement, avec des coûts fixes et amortissables durant toute la durée d'exploitation; et, d'autre part, le bas de tableau consacré à l'exploitation: des dépenses qui augmentent avec le nombre d'inscrits (impressions, correction de devoirs, etc.) et surtout qui se répètent pour chaque année où le dispositif fonctionne. Il va de soi qu'il est demandé aux concepteurs de formation de maximiser les investissements et de minimiser l'exploitation, afin d'être plus rentable et de mieux contrôler les dépenses.

Ceci se traduit, par exemple, par une automatisation des corrections sous forme de questionnaires à choix multiples à correction informatique : un investissement supplémentaire est fait pour avoir un lot de sujets varié avec des réponses types à entrer dans un logiciel informatique adapté. Ceci appelle plus de frais lors de la conception du dispositif, mais la diminution des coûts de correction, d'année en année et quel que soit le nombre d'inscrits, permet d'amortir rapidement l'investissement. À l'opposé, la pression pour mettre en place des formations hybrides, faisant intervenir des formateurs en présence pour une plus grande qualité pédagogique, fait passer du « haut de tableau » (investir dans un fascicule de cours à distance réutilisable plusieurs années et imprimable à faibles coûts) vers le «bas de tableau » (le formateur doit revenir chaque année et est payé pour chaque prestation).

Face à cette injonction de la direction de préférer l'investissement à l'exploitation, les concepteurs de formation sont tentés de faire glisser, par jeu comptable, des éléments de bas de tableau vers le haut de tableau, c'est-à-dire faire passer pour de l'investissement ce qui est de l'exploitation afin de paraître plus rentable. Ainsi, sur le terrain, nous avons assisté à une réaction des concepteurs de formation qui, collectivement, ont imposé au contrôle de gestion produisant l'outil comptable la ligne « réingénierie » comprenant la mise à jour des fascicules de cours chaque année. Il s'agissait d'un groupe de responsables de formation chargé d'ingénierie de formation travaillant chacun à la préparation des concours d'agrégation de Capes dans leur discipline. Une partie du travail était, dans ce dispositif de formation stabilisé (les types de clients et l'infrastructure de la préparation aux concours restent identiques d'une année à l'autre), est de renouveler des contrats d'auteurs avec des vacataires pour s'adapter aux programmes changeants de l'Éducation nationale. Considérer que ce travail était de la «réingénierie » (terme inventé par ce groupe d'employés) et le faire figurer en investissement permettait de valoriser par jeu comptable leur activité. Ce alors qu'il est possible de considérer qu'il s'agit d'un réinvestissement, c'est-à-dire d'un investissement annuel et donc d'un coût d'exploitation. 


\section{L'analyse financière : les seuils}

La troisième feuille de calcul du tableur présente l'analyse financière mettant en regard les recettes issues de la mercatique avec les dépenses comptabilisées. Cette feuille synthétique offre une vue sur l'ensemble de la période d'exploitation de l'amortissement des investissements (divisés par le nombre d'année). Elle permet de déterminer des seuils comme le nombre minimal d'inscrit pour que la formation soit rentable (c'est-à-dire que le revenu soit supérieur aux dépenses), le nombre minimal de session pour que la formation soit rentable ou encore le prix minimal. Ainsi le personnel peut faire varier tel ou tel paramètre de la feuille de calcul pour afficher tous ces indicateurs en vert. Certes le prix est vérifié par un service support de la direction commerciale (un price analyst) mais les propositions des concepteurs de formation sont déterminantes.

Un taux de rentabilité compris entre quarante et soixante-dix pourcent est le plus fréquent, quoique, comme nous l'avons montré dans les paragraphes précédents, un acte unilatéral administratif de la puissance publique peut imposer à l'organisme public de créer un dispositif non rentable - et inversement, des produits exceptionnels peuvent se montrer très rentables faute de concurrence sur le marché.

Ainsi, le concepteur de formation a à son actif un portefeuille de formations qu'il peut mesurer et faire valoir avec un chiffre d'affaires (et non pas simplement un nombre d'inscrits), une marge totale et un taux de rentabilité moyen. Ceci afin de défendre sa bonne gestion lors des entretiens annuels d'évaluation et permettre sa progression dans la hiérarchie de l'organisation.

\section{Conclusion : perspectives distanciées sur la notion de valeur}

Nous avons tenté de montrer, dans les chapitres précédents, comment les sciences de gestion étaient instrumentées et devenaient des pratiques légitimes pour concevoir les formations. Nous soutenons que l'étude de ces pratiques fait partie des sciences de l'éducation (à titre de sociologie des pratiques de gestion éducative, voire d'outils d'apprentissage de l'ingénierie de formation). La culture multidisciplinaire des sciences de l'éducation impose cependant de revenir à un regard complexe et distant par rapport à ces outils et la logique du tout économique qu'ils instillent. Nous allons ainsi présenter des critiques de terrain mais aussi celles, classiques, de la littérature. Pour compléter ce que nous avons mentionné concernant les rapports de forces pour imposer les valeurs de tel ou tel service 
ou personnel dans les documents arrêtant les procédures d'évaluation, nous commencerons par avoir recours à la critique économique de Karl Marx en développant la notion de valeur d'usage.

\section{La valeur d'usage : vers l'experience d'apprentissage}

Au chapitre «Marchandise et monnaie» du Capital (Marx, 2008), nous relisons : «Le caractère utile d'une chose en fait une valeur d'usage» puis «La valeur d'usage ne se réalise effectivement que dans l'usage ou la consommation ». Autrement dit, il apparaît que le calcul de la valeur monétaire du «produit de formation» par le concepteur qui le fabrique est discutable. Tout d'abord parce que le produit est peut être, contrairement au langage du terrain, un service (d'accompagnement, de guidage, d'orientation : un service pédagogique) mais aussi et surtout parce que celui qui en détermine la valeur est d'abord celui qui en a usage, c'est-à-dire le consommateur apprenant lors de la session de formation. Un décentrement du calcul de la valeur devrait ainsi mettre l'apprenant au centre (comme le recommande l'Union Européenne par le processus de Bologne) pour comprendre quels sont ses gains (immédiats ou différés) et ses coûts (en argent, en temps ou en effort ?) lorsqu'il apprend. Il semble alors qu'il serait préférable de construire le modèle de la valeur, à l'image de Gary Becker (1984), sur l'idée d'un capital humain dans lequel investissent les apprenants pour s'enrichir. Mais force est de constater que ces considérations économiques ne sont pas encore utilisées sur le terrain des organismes étudiés où le décentrement ne s'opère que par la pratique et les retours d'expérience des coordinateurs de formation: aucun modèle gestionnaire n'est proposé permettant d'intégrer l'usage et la valeur qu'a la formation pour celui qui y consacre du temps, de l'énergie et de l'argent. Tout se passe comme si le concepteur, marchand et fabriquant de la formation, ne connaissait pas très bien ses clients et leur donnait un produit sans se soucier de l'utilité réelle qu'elle aurait pour eux. Tout au plus un effort est fait sur le terrain pour rendre positive l'expérience de formation et ainsi en diminuer le coût : une tendance au ludique tente de transformer la formation en jeu sérieux prenant en compte l'esthétique et la sensibilité de l'apprenant. Cet effort ergonomique dans les dispositifs de formation passe par une relation individualisée de meilleure qualité mais aussi un sens de «l'emballage » où le produit de formation est présenté sous son meilleur jour pour laisser entrevoir une expérience de formation de qualité et espérer des usages multiples la valorisant.

\section{Evaluation et valuation : reconnaître les valeurs professionnelles}

Nous avons vu comment l'évaluation monétaire de la formation par les outils de sciences de gestion passe, sur le terrain, par des rapports de force (ou valuation) : chacun essaie de faire entrer ses 
propres valeurs dans le système auquel se réfère l'évaluation. La difficulté pour trouver un langage simple est que, lorsque l'on considère l'abstraction «valeur » dans ses manifestations concrètes sur le terrain, on se rend compte qu'elle change selon qui évalue et à quelle fin : est-ce le concepteur de formation pour optimiser son portefeuille? Le client de l'entreprise pour augmenter sa force productive ? Le consommateur pour augmenter son employabilité ? La même formation a ainsi plusieurs évaluateurs aux objectifs variés et donc plusieurs valeurs car ils ne parlent pas tous la même langue. La valeur marchande et la valeur de fabrication sont par exemple différentes de la valeur d'usage de la formation.

Nous laisserons le dernier mot aux sciences de l'éducation, et, au-delà de l'anti-économisme, voire du refus de principe de la réduction à un chiffre ou une monnaie uniques qui existent dans certains cas sur le terrain, nous reprenons les travaux de Richard Wittorski (2007) sur la professionnalisation et Anne Jorro (2007) sur la reconnaissance : postulant que la formation n'est pas un loisir qui est une fin en elle-même, nous la regardons ici comme un moyen d'être reconnu par une profession. Alors l'évaluation de la formation passe par l'intégration, dans son système de références, des valeurs de la communauté professionnelle visée. Et, de fait, nous avons pu constater sur le terrain que les concepteurs de formation de gestion, comme leurs apprenants se destinant à ces professions, étaient plus sensibles au discours gestionnaire pour évaluer la formation - qui n'est que le discours d'une des communautés des professionnels de l'éducation.

\section{La carte commerciale : un outil de marketing de la formation}

\section{$\underline{\text { Introduction }}$}

Dans la lignée des récits d'étude institutionnels, un magazine en ligne - ou webzine présente des chemins de formation atypiques qui ont été suivis grâce à un établissement public d'enseignement à distance. Nous proposons une analyse de ce webzine institutionnel.

Notre démarche se fonde sur des données empiriques recueillies par observationparticipante dans les bureaux de l'institution d'enseignement à distance et notamment la réunion de présentation du webzine aux employés par son créateur, rattaché à direction commerciale. Nous nous appuierons aussi sur un commentaire, article par article, du site Internet tel qu'il parait en ligne le 8 décembre 2012 : l'analyse des articles de journalisme documenté qu'il contient fera l'objet de notre première partie. 
Ce matériau empirique sera travaillé avec les outils conceptuels des sociologues des récits de vie (Bertaux, 1996, Bourdieu 1986) et, plus largement, par l'interactionnisme symbolique qui s'attache aux jeux de présentation (Goffman, 1991). Nous aurons recours aux analyses de Jean-Marie Barbier (Barbier, 2011) et de l'équipe du Centre de recherche sur la formation du Cnam pour comprendre ces récits comme une activité de gestion de la formation : la direction commerciale de l'institution d'enseignement fait œuvre de marketing de la formation en communicant au mieux sur ces trajectoires de formation réussies pour créer une communauté en ligne et ainsi vendre des formations.

Nous procèderons en trois temps : d'abord le commentaire du site Internet, pour ensuite cartographier les chemins atypiques de formation présentés (leur représentation imagée est racontée selon un mode narratif particulier) et enfin comprendre la dimension commerciale de cette carte de visite (un acte de gestion pour mieux vendre la formation).

\section{Commentaire du webzine}

Lorsque nous parcourons la page d'accueil du webzine, nous découvrons d'emblée des photos et un logo hauts en couleurs, qui frappent l'œil. En haut à gauche, la marque mêle le vert pomme au rose vif, puis une série de photos qui défilent, avec des titres accrocheurs : « Au bout du monde », « Success story »,...

Le créateur du webzine annonce aux employés de l'institution, en octobre 2012, que le titre du site Internet renvoie à la fois au mot anglais « smooth» et au verlan de « zooms ». En effet, poursuit-il, le site Internet veut être une série de zooms sur des trajectoires d'études hors du commun suivie avec l'institution d'enseignement à distance. D'où l'effet de vertige pour l'Internaute, à la vue de ces photos aux couleurs chamarrées, sur fond de coucher de soleil et de voilier voguant vers le grand large.

Le slogan «Votre avenir est notre histoire », ou, un peu plus loin « Le site qui vous raconte des histoires d'avenir », rappelle le slogan de l'institution ("Connecté à votre avenir $\gg$ ) et joue sur les confusions de temps, entre passé, présent et avenir pour viser l'atemporel, ou peut être le fait que les auteurs ont un temps d'avance sur les lecteurs cibles. La plupart des articles présentés sont en effet écrits au passé composé et racontent l'avenir éventuel d'un inscrit potentiel - qui vient rêver devant ces trajectoires possibles ainsi mises en vitrine. La promesse du site est la suivante : «Depuis plus de 70 ans, l'établissement vous aide à préparer votre avenir. Voilà qui promet de jolis zooms... ». Autrement dit, ce que d'autres étudiants ont fait par le passé, qui est le quotidien l'institution, sera votre avenir si vous la rejoignez pour apprendre dans ses programmes. 
Dans la rubrique servant de plan du site, l'internaute peut lire : "Résolument tourné vers vos projets, le webzine est la passerelle idéale qui vous permettra d'anticiper votre avenir. Quel que soit votre objectif, vous trouverez forcément des pistes pour le préparer au mieux.". La métaphore de la piste, du chemin ou de la trajectoire de formation, est donc bien présente.

Pourtant, le site, avec ses nombreuses images de sports extrêmes (escalade, VTT, snowboard...) et d'aventures familiales exceptionnelles (tour du monde en voilier, vie de saltimbanques,...) décrit des chemins de formation hors du commun, des trajectoires horspistes, hors des sentiers battus. Il présente, en deux mots, des chemins atypiques.

Le site montre des images inimaginables à l'Internaute, pour lui ouvrir des perspectives d'études inattendues, dans des lieux insolites. La prise de distance avec le quotidien permet de faire entrer dans notre ordinaire un peu d'extraordinaire : de l'exotique, du passionnel et du succès. Le webzine fait connaitre des chemins de formation inconnus, repousse les frontières du méconnu et invite au voyage et donc associe loisirs et études.

Le hors-piste en formation passe par le hors-classe : le cliché du sportif solitaire ou de l'explorateur qui refuse le chemin grégaire du troupeau et lui préfère la formation individualisée, tracée par soi et pour soi - avec l'aide de l'institution. La formation «surmesure » (selon la langue vernaculaire) relève du hors-série, de ce qui ne se mesure a aucun standard type, qui n'est fabriqué qu'en un exemplaire unique.

Le site Internet est ensuite composé de plusieurs rubriques, nourries de vidéos et de nombreux liens hypertextes vers les blogs des étudiants aventuriers : il s'agit d'un document hypermédia hautement interactif, dans la lignée de «l'écriture numérique » recommandée par l'institution à ses équipes pédagogiques (notamment dans le document de développement stratégique remis aux employés en novembre 2012).

Voyons à présent deux exemples de chemins atypiques tels qu'ils sont décrits dans les articles du site Internet et qui nous paraissent constituer des archétypes.

\section{Une carte topographique : deux chemins de formation atypiques}

Nous soutenons que les différents récits de formation que l'on peut lire dans le webzine constituent une carte topographique d'un imaginaire bien particulier : celui des chemins de formation hors-classe, suivi avec une académie en ligne. La page de plan du site présente une image qui conforte notre thèse : celle d'une carte des itinéraires de formation. C'est une mappemonde avec une boussole. Le webzine ne donne pourtant pas une carte scientifique des itinéraires de formation : malgré des données objectives relevant de la sociologie (profession, âge, genre, nombre d'enfants des aventuriers scolaires mentionnés à 
chaque début d'article), il semblerait que ce soit l'imaginaire de l'Internaute, ses rêves et ses désirs, que visent les itinéraires de formations présentés.

Le site présente un ensemble de clichés et de brèves histoires de vie de «people » qui montrent des itinéraires dissemblables, réunis par leur seul lien commun : celui de l'institution d'enseignement à distance. Ainsi défilent sur l'écran un voilier (l'histoire d'une famille partie faire le tour du monde), un surfer (l'histoire d'une jeune championne de France du surf), d'un féru d'escalade, d'un compétiteur de karting, d'un jockey... Nous choisissons de les regrouper en deux archétypes de l'atypique ces figures emblématiques du sportif ou du voyageur au chemin de formation singulier : d'une part l'aventure familiale et d'autre part l'exploit individuel.

\section{L'exploit individuel}

Le premier article qui retient notre attention est un reportage vidéo qui apparaît en première page, dans le bandeau dynamique défilant à l'écran. Le reportage montre une jeune fille de quinze ans qui vit sa passion du surf en accord avec des études par correspondance. Après plusieurs belles images de surf, l'Internaute peut visionner un entretien avec l'élève qui raconte les deux entraînements par jour (et donc l'impossibilité d'aller au lycée) et la nécessité d'un «mental» pour tenir la distance (elle vient de Guadeloupe). Le reportage montre quelques chutes dans l'eau et le mot «infaisable» est prononcé. Mais l'erreur est tolérée et la jeune fille remonte sur le surf pour surmonter les remous et les difficultés qui déferlent. Suit un entretien avec son père qui explique, rassurant, que sa fille fait partie du pôle espoir surf dont il s'occupe. Les images montrent les préparatifs de sa fille avant qu'elle ne se jette à l'eau : elle referme sa combinaison, s'échauffe et prend son surf sous le regard bienveillant de celui qui l'accompagne à distance. Elle revient à l'écran pour affirmer «le brevet j'ai trouvé facile (...) avec l'enseignement à distance c'est beaucoup plus facile de passer un brevet que si on était à l'école », puis son père rappelle la flexibilité de l'emploi du temps pour suivre la météo des vagues capricieuses et pour se déplacer sur les lieux de compétitions, il complète : «Les jeunes ont de très bons résultats, ils gagnent en autonomie dans leur travail ». Et le générique s'achève sur une image de surf sous-titrée : «Le 31 octobre 2012, Kim est devenue championne de France espoir de Surf ».

Il apparaît ainsi clairement que l'exploit individuel de la jeune lycéenne (ce qui appelle au rêve) est soutenu par le père responsable (qui rassure les parents dans le projet d'études) et, en filigrane, l'institution qui permet flexibilité et adaptation à l'itinéraire singulier. La vidéo présente donc un ensemble d'acteurs engagés pour assurer la réussite de celle qui tient le 
premier rôle. Les spectateurs, à la fois étonnés et rassurés, se prennent à imaginer à un itinéraire de formation tracé pour eux-seuls.

\section{L'aventure familiale}

À ce premier archétype de l'atypique nous voulons ajouter le miroir complémentaire : l'aventure familiale. C'est là un itinéraire collectif, qui est souvent porté par le rêve individuel d'un père ou d'une mère qui veut faire partager sa passion à sa famille.

La rubrique du site « au bout du monde » annonce : « en camping-car, en bateau ou à dos de chameau, un jour, ils ont décidé de partir à l'autre bout du monde pour voir si la terre est ronde ». Nous prendrons ici l'exemple d'une famille partie faire la traversée de l'Atlantique en voilier. Le reportage vidéo montre la famille sur le voilier (avec un lien vers leur blog) et des articles parallèles recueillent l'avis de la grand-mère, restée à quai et pourtant enthousiaste à l'idée de cette aventure. Un expert de l'institution d'enseignement est interrogé et ramène le voyage à un ensemble de formalités administratives pour pouvoir inscrire ses enfants à distance. Quelques anecdotes racontent la poste restante, les communications Internet, la vie dans le cockpit alors que le père tient le cap et finalement les très bons résultats scolaires des quatre enfants. Dans cet itinéraire de formation, c'est le père qui tient la barre et l'ensemble de la famille qui lève l'ancre pour voguer vers le grand large.

Les enfants « tourdumondistes » deviennent une catégorie à part entière, un archétype, et le reportage s'achève sur un lien vers le site Internet de vente de formation et le numéro de téléphone du télé-accueil, en charge de la prospection des clients. Nous touchons alors à une dimension importante du site : cette topographie des itinéraires de formation atypiques semble être une carte commerciale non négligeable pour l'institution publique.

\section{Le webzine : une carte commerciale pour l'enseignement à distance?}

Sous le couvert de journalisme documenté, c'est en effet un discours de marketing de la formation qui se dissimule. Le but de la revue institutionnelle est de donner envie d'apprendre à distance et de s'inscrire. Avec le webzine, l'institution d'enseignement joue donc une carte commerciale pour vendre de la formation en communiquant sur des profils atypiques auxquels s'identifier. Nous pensons donc qu'il s'agit d'un acte de gestion: l'atypique revient à un fonds de commerce et une bonne communication marketing permet de le vendre au mieux.

Chaque récit de formation est ponctué par des allusions aux avantages de l'institution : le webzine exploite les exploits d'études avec un discours marketing qui se compose de phrases courtes, écrites à la troisième personne et à l'indicatif, marquées par des points 
d'exclamation. Les images et ce style particuliers appellent au rêve sans juger subjectivement : l'indicatif montre objectivement et l'Internaute s'exclame avec l'auteur. Les données socioprofessionnelles sont là pour donner un semblant de journalisme documenté et véridique. Une structure du discours revient dans chaque article : l'image qui fait rêver, les difficultés de l'itinéraire, et enfin l'exploit grâce au à l'institution salvatrice. Ce style, ces images et cette structure accrocheurs sont un outil de marketing pour vendre du rêve, ou du moins présenter la formation sur le mode onirique.

Nous pouvons conforter ceci en soulignant la dimension collective du rêve. Une section du site s'intitule « la cafet' ». Nous pouvons y entrer librement en nous inscrivant dans les bases de données. Plusieurs forums s'offrent alors au visiteur curieux, où sont débattus des thèmes comme «le welcome café », « réussir sa scolarité », « examens et concours », « enseignement à distance », « recherche de stage et emploi », «vivre à l'étranger » et enfin «le coin des $\mathrm{D}^{42} »$. Un « $\mathrm{D} »$ est une catégorie qui désigne un inscrit ou un employé de l'institution. Cette identité collective renforce l'adhésion. C'est dans ces forums que se crée la convivialité qui manque à l'enseignement à distance : les inscrits et clients potentiels débattent comme dans une cour de récréation où les classent se mêlent. L'informel prend forme et les récits d'études extraordinaires circulent spontanément.

Les débats $\mathrm{du}$ forum ne sont cependant pas totalement libres : une community manager est là pour les modérer et répondre aux questions pratiques. Elle se présente par un mot de bienvenue qui annonce : «Vous souhaitez suivre une formation à distance et vous avez des questions ? La cafet' est faite pour vous :) ». Le ton informatif et le sourire électronique annoncent bien le lieu de recrutement où l'on vend du rêve de formation, avec ses clichés et ses connexions promises à l'emploi et au succès. La rubrique donne en effet plusieurs conseils pratiques pour réussir et un partenariat avec un site de recherche d'emploi.

\section{Conclusion}

Le commentaire du webzine nous permet de comprendre que la carte de l'imaginaire de formation atypique est aussi une carte commerciale bien réelle. Le ski, l'équitation, le surf, le snowboard, le voilier ou le cirque familial sont tour à tour utilisés pour vendre du rêve. Et à chaque occasion l'institution est mise en valeur : la rigueur, l'organisation, l'autonomie ou encore la responsabilité sont des valeurs qui reviennent au détour de nombreux articles.

\footnotetext{
${ }^{42}$ Nous anonymisons le terme « D »
} 


\section{Le « projet G. » : le geste et son effet dans la conception de formation}

Nous présentons, en spectateur averti, un projet d'ingénierie de formation dans le cadre d'une institution d'enseignement française opérant en Europe - dont nous sommes acteur. Le projet d'ingénierie consiste à développer une école de gestion en ligne (anonymisée par l'appellation «projet G. »), d'une durée de trois années, visant un public d'une soixantaine de néo-bacheliers, avec une équipe pédagogique d'une dizaine d'enseignants employés par le Ministère de l'Education nationale.

Décrire ce projet, en cours de déploiement au moment de la description mais qui a finalement été abandonné par l'institution, va nous permettre de présenter l'organisation et l'ingénierie de formation, par l'utilisation de la gestion en sciences de l'éducation.

\section{Du geste à l'effet}

Le Dictionnaire étymologique et historique du français Larousse (2001) dérive le mot gestion $\mathrm{du}$ latin gerere : le faire, l'action fondent la gestion ${ }^{43}$. Une histoire des sciences de la gestion s'enracinerait ainsi dans la phénoménologie de l'action du philosophe Maurice Blondel ${ }^{44}$ au siècle de l'industrie, sortirait de terre avec les économistes praxéologistes, dont l'illustre Ludwig von Mises ${ }^{45}$, et fleurirait au $20^{\text {ème }}$ siècle ${ }^{46}$. Mais c'est l'épistémologie de la gestion qui nous retient, avec le geste et l'effet comme type causal premier de la logique de l'action.

Quels sont les bons gestes qui produisent les bons effets ? Les gestes efficaces et effectifs de l'employé confirmé ? Et non les gesticulations défectueuses du débutant ? En quoi certains actes de conception des experts relèvent de la gestation et produisent des effets gigognes ? Cette dialectique du geste et de l'effet est à l'œuvre dans le projet d'ingénierie de formation que nous exposons, nous soutenons que c'est un implicite de la pensée gestionnaire.

Actes et actants : les routines de la gestion de formation

Quels sont les actants concernés par le projet d'ingénierie de formation ? Le projet compte d'abord des responsables de formation, c'est-à-dire des gestionnaires, qui agissent en coordonnant différents métiers au sein de l'institution d'enseignement. Production des supports pédagogiques, commercialisation des produits de formation, administration des inscriptions, innovation dans les dispositifs, appartiennent chacun à une direction métier et le responsable de formation, en charge d'un

\footnotetext{
${ }^{43}$ Voir aussi les travaux de la FNEGE pour centraliser les sciences de l'action

${ }^{44}$ Blondel M., 1993, L'action, PUF. Quadrige. France.

${ }^{45}$ Mises L. von, 2004, Abrégé de l'action humaine, Belles Lettres. France.

${ }^{46}$ Une histoire des sciences de la gestion dans la première moitié du $20^{\mathrm{ème}}$ siècle est esquissée dans le travail sociologique de Boltanski L., 1982, Les cadres. La formation d'un groupe social, Éditions de Minuit. Le sens commun. Paris.
} 
ou plusieurs produits de formation, correspond avec des interlocuteurs dans chacune de ces directions métiers. En d'autres termes, il agit en chef de projet effectuant du management transversal.

Les responsables de formation, dans le site de l'institution que nous observons, sont issus du personnel de l'Education nationale : certifiés ou agrégés dans leur discipline, parfois docteurs, ils ont évolué vers cette fonction à responsabilité après quelques années d'enseignement. Contrairement au personnel de direction classique, proviseur de lycée ou principal de collège, ils ne sont pas chef d'un établissement. L'ampleur de leur tâche est donc moindre, quoique les moyens d'action au service de l'innovation pédagogique soient plus nombreux: ils ont pour charge d'assurer l'ingénierie de formation, c'est-à-dire de proposer sans cesse de nouveaux dispositifs de formation dans leur périmètre d'activité.

Les responsables de formation œuvrent de leur chef et de leur main puisqu'ils agissent de la conception du projet jusqu'à sa réalisation et son suivi. Nous nous centrerons sur le chef-d'œuvre (la conception) mais n'oublions pas que les responsables de formation sont aussi main-d'œuvre: ils signent les attestations de formation, accompagnent la commercialisation en téléphonant aux apprenant pour mieux les comprendre, assurent la réponse aux questions pédagogiques sur les forums des sites Internet de formation, garantissent l'absence de faute dans les supports de cours par des relectures attentives.... Certes ils ne font pas tout : ils font faire une bonne partie du tout en déléguant. Mais la responsabilité leur revient toujours et ils sont amenés à vérifier sans cesse, à «attester du service fait » des nombreux personnels vacataires auxquels ils ont recours ponctuellement.

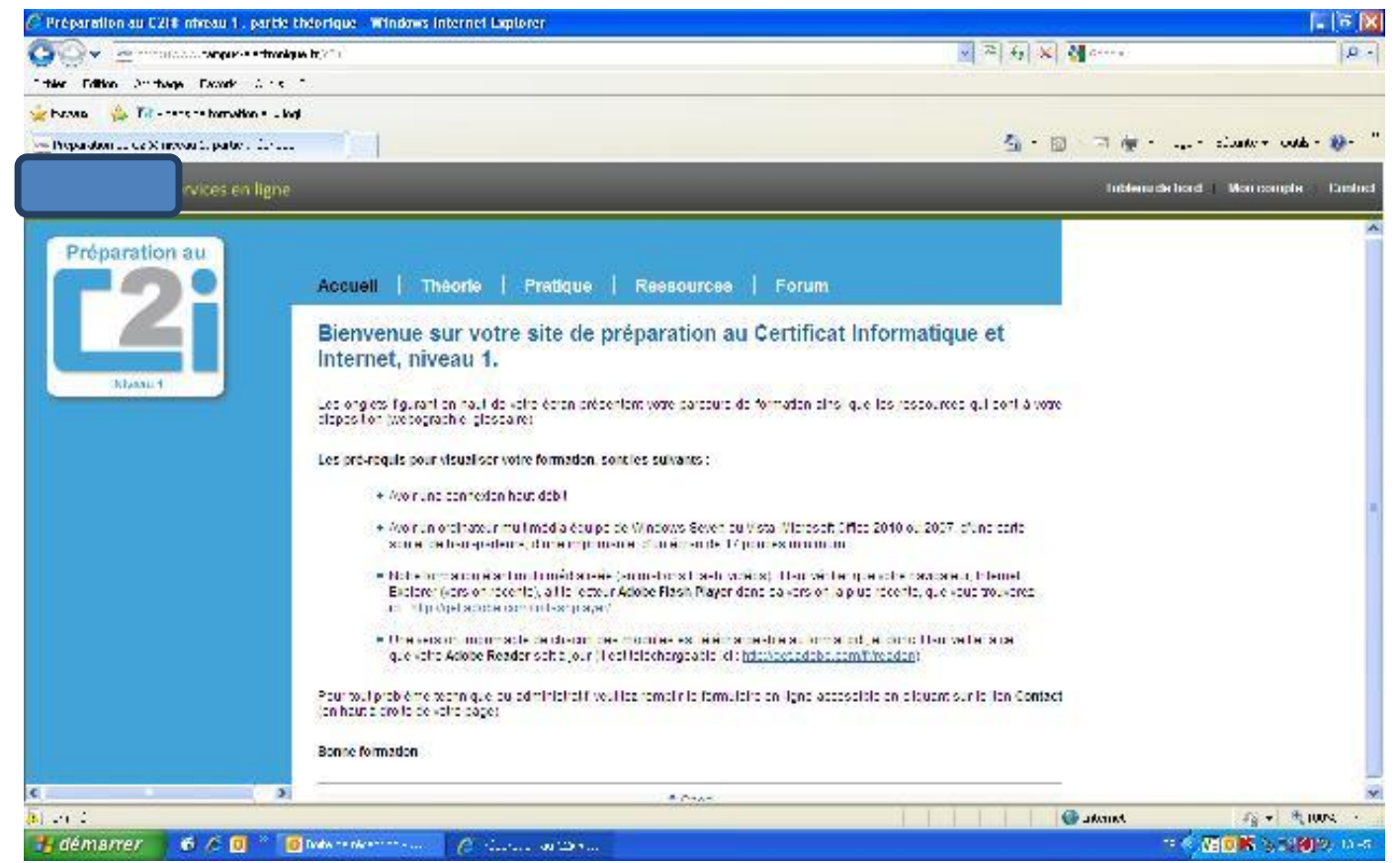


Comment s'effectue l'ingénierie de formation? L'idée de nouveau dispositif vient rarement d'un seul responsable de formation isolé. Le retour par téléphone d'un inscrit, une direction des ministères dont dépend l'institution, une réunion informelle avec des collègues le temps d'une pausedéjeuner, l'imitation d'un concurrent... sont autant d'éléments déclencheurs. La valeur ajoutée du responsable de formation chargé d'ingénierie pédagogique consiste alors à entrer dans les formes de l'institution pour se conformer aux méthodes de travail de ses collègues et donner ainsi une ampleur collective à ses projets.

Un geste créatif, la bonne idée, ne sera effectif que s'il a prise sur les réalités de l'institution. Une idée de formation n'est que du vent si elle est simplement prononcée au détour d'une conversation, à la pause-déjeuner. L'idée de formation devient réelle si elle prend une forme assez dure pour durer. Cette forme solide peut être un document papier, qui laissera une trace dans les archives et revivra à chaque relecture ; ou encore un groupe de travail, avec un calendrier et des actes de réunion qui autorisent les décisions prises. Le geste trouve alors son effet : un effet solide dans le cas d'un écrit (c'est l'effet ricochet: l'idée rebondit dans tous les esprits qui la lisent), un effet instituant dans le cas d'une réunion (c'est l'effet boule de neige, l'idée comptant de nouveaux adeptes au fur et à mesure que le groupe de travail grandit).

La nouvelle formation est elle-même comprise comme un « dispositif » : c'est un ensemble de cours (sur support papier ou numérique), de procédures (d'inscription, de circuit des copies, d'évaluation notée) et de règles (interactions des apprenants avec les tuteurs par téléphone ou sur le site Internet) qui constitue un objet. L'emballage commercial (le marketing du public visé, prix affiché, promotion envisagée, lieu de vente) et institutionnel (discours sur le contenu, partenariats avec d'autres institutions) en font un produit de formation complet. Le responsable de formation n'encadre pas directement des enseignants et des élèves; il s'ingénie à créer des objets physiques qui cadrent indirectement les actions humaines pour lui. L'institution, machine constituée de procédures et de formes écrites, autorise les actes du responsable de formation. En réglant la machine par des gestes précis et idoines, il s'assure qu'elle formatera les esprits des apprenants. 


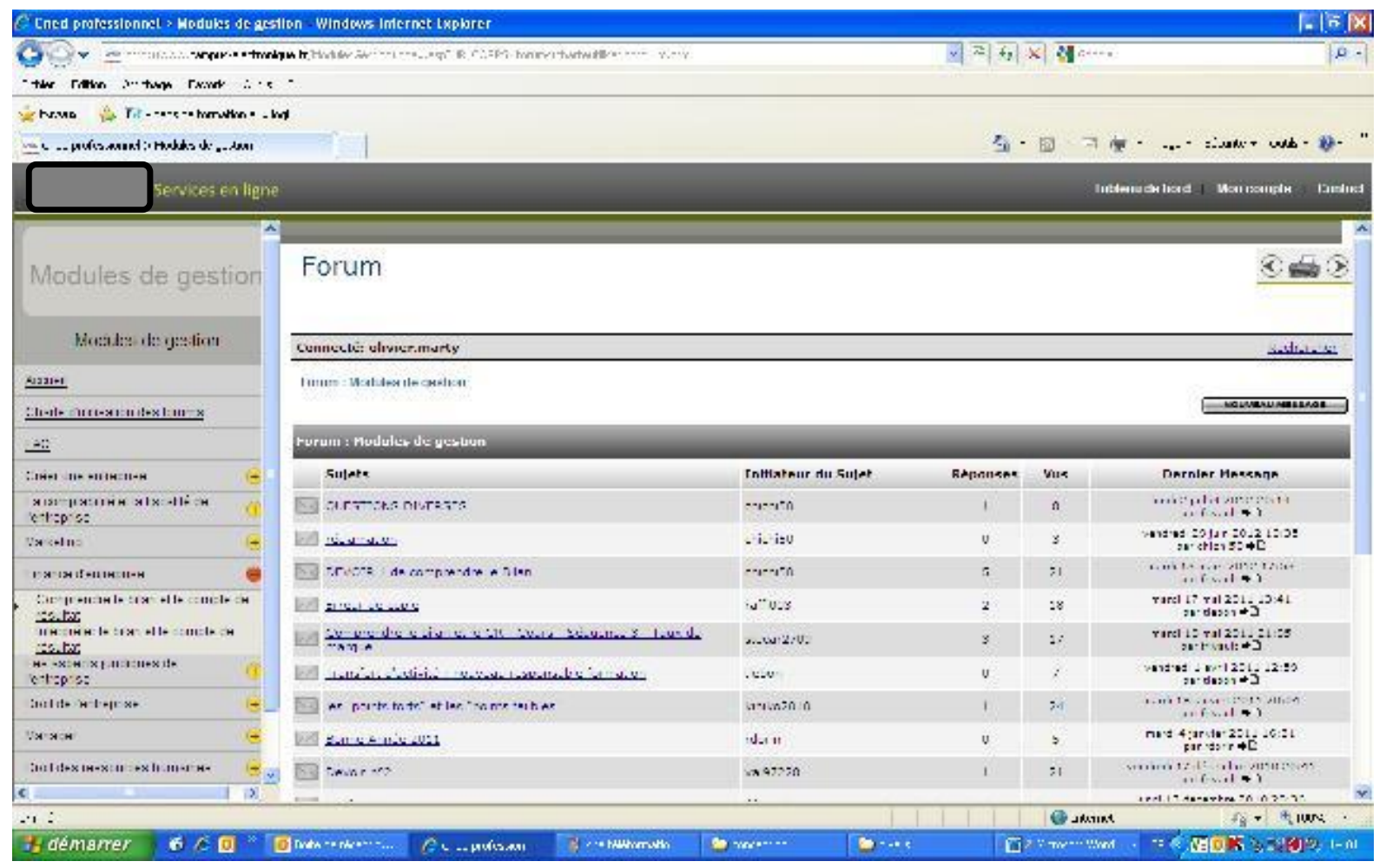

\section{Exemple d'actant contenant les gestes des acteurs : le forum d'un site de formation}

Comme nous le voyons, les responsables de formation, acteurs humains chargés d'ingénierie de formation, sont insérés dans un réseau d'acteurs non-humains: écrits, groupes de travail (organisation, procédure), actes de réunion (formalités)... Il n'est pas anodin que la théorie de la traduction $^{47}$, réunissant actants humains et non-humains, soit présente en annexe d'un ouvrage technique à destination de ce public ${ }^{48}$. Les professionnels de la formation reconnaissent que la bonne idée a plus de valeur si elle s'impose «par la force des choses » au lieu que de ne reposer uniquement sur la volonté des hommes. Les humains s'entourent d'inhumains pour que leurs gestes aient des effets surhumains. La gestion s'immerge dans le sociotechnique. Ou encore, la culture d'action ${ }^{49}$ de l'institution mêle des hommes et des objets, elle est à la fois humaine et matérielle.

\footnotetext{
${ }^{47}$ Latour B., Callon M., 2005, La science en action : introduction à la sociologie des sciences, La découverte. France.

${ }^{48}$ Annexe de "Outils de pilotage des systèmes éducatifs", séminaire de la Mafpen et de I'lufm de l'académie de Lyon, 1995, textes coordonnés par Jacques Jumentier, CRDP de l'académie de Lyon

${ }^{49}$ Barbier JM, 2010, "Cultures d'action et modes partagés d'organisation des construction de sens ", Revue d'anthropologie des connaissances.
} 
$\underline{\text { Une rupture innovante : transformations de la culture d'action éducative }}$

Le projet d'ingénierie de la formation d'une école de gestion («Projet $G$ ») retient notre attention en tant que geste inchoatif ou de gestation avortée sous les yeux de l'observateur. Nous sommes dans le cas des balbutiements d'une innovation: il ne suffit plus de s'assurer du fonctionnement de produits de formation existant, ou de répliquer une formation modèle pour en créer une nouvelle (de type BTS, licence ou master) mais bien d'innover par une formation atypique dans l'institution. À effet nouveau, geste nouveau : le projet G. signale plusieurs changements de fonds dans la culture d'action éducative de l'institution étudiée.

Comme la montré Jean Marie Barbier, la culture d'action éducative est d'abord partage d'un sens commun. Elle est, pour nous, manichéenne : elle sépare le «bon geste» du «mauvaise geste » d'un point de vue déontologique comme d'un point de vue pratique. Le « mauvais effet» est aussi bien la vision d'un mauvais geste que celle de ses conséquences. Opposant « ce qui se fait » à «ce qui ne se fait pas », voire à «ce qui ne le fait pas », la culture d'action éducative définit des obligations et des interdits. Comment opère-t-elle dans le cas du projet G. ?

Le responsable formation chargé d'ingénierie pédagogique est supposé faire preuve d'une éthique professionnelle vouée à la connaissance (trahie par le paradoxe du « je ne sais pas » comme réponse courante, qui signifie en réalité « je sais beaucoup de choses mais ceci je ne le sais pas »), désintéressée (on ne parle pas d'argent et de rémunération dans les couloirs de l'institution étudiée) et affichant le service public à tout va (la langue vernaculaire, proscrivant l'anglais international et prescrivant le français recherché, parle volontiers «d'apprenant», «d'inscrit» mais jamais de « client », à la différence d'organismes de formation privés).

Or le Projet G. est le signe d'une transformation de cette culture d'action éducative. Il est censé être rentable à court terme ; il se positionne sur un marché dominé par des écoles privées et doit donc adopter leur logique ; il est, enfin, mené par un responsable de formation qui a été formé dans une grande école de commerce privée. Là où les responsables de formation étaient quasi exclusivement issus de l'Education nationale, une nouvelle vague de recrutement a fait entrer dans l'institution un personnel issu de grandes écoles de commerce et ayant travaillé au préalable dans le secteur privé. À nouveau projet d'ingénierie de formation, nouvelle culture éducative.

Les hommes changent et avec eux les valeurs (l'argent, le profit s'introduisent subrepticement), les normes et règles de mesure (compter son «investissement» au plus près, rentabiliser). Ceci passe, au niveau de la direction générale de l'institution, par la création d'un service de « contrôle de gestion ». Ce nouveau service, en contrôlant la gestion des responsables de formation, va faire plus qu'inspecter les gestes des uns et des autres : il introduit des manières d'agir et de penser 
propres au secteur privé. Les actions sont quantifiées, mesurées par des tableaux de calcul sous format Excel et finalement encadrées par cette nouvelle raison numérique ${ }^{50}$.

Le projet G. révèle la transformation vers un nouveau sens commun : des valeurs chiffrées, des mesures et des unités de mesure qui font sens (alors qu'elles étaient incomprises auparavant, insensées), des règles de calcul communes. C'est une nouvelle politique qui prend forme: l'établissement public hésitant dans son ensemble à passer du statut d'Établissement public administratif à celui d'Établissement public industriel et commercial. Le geste d'ingénierie du projet G. est donc un geste qui va dans le bon sens : il n'est pas à contresens de l'évolution de l'institution, il suit la direction du vent de l'histoire.

Geste complet, le projet G. mobilise les actants de l'ancienne et de la nouvelle génération (procédures, responsables, services, documents officiels) pour produire les bons effets : formation conforme à la culture d'action commune, aux représentations que l'institution se fait de ce qui doit être fait et de comment cela doit être fait. Ainsi le projet G. est en harmonie avec l'institution dans ce qu'il définit être les attentes des apprenants : ce qui mérite d'être appris (et qui s'inscrit dans cette culture gestionnaire chiffrée, internationale, du geste efficace), le niveau de qualité pédagogique espéré ou encore le prix qui doit être à la fois fidèle à la tradition de service public (abordable par tous) et rentable.

\section{$\underline{\text { Temps et procédures d'action }}$}

Le projet G. est un processus qui est scandé par des formes propres à l'institution. Chaque étape du développement du projet d'ingénierie de formation est marquée par un document qui doit être validé par le comité de direction idoine. Le produit de formation a ainsi un cycle de vie qui débute avec une fiche de proposition de nouvelle formation, suivie d'une fiche de conception de la nouvelle formation (impliquant les différents métiers de l'institution : production, commercialisation, scolarité, innovation) et ainsi jusqu'à une fiche de suppression de la formation. Le responsable de formation doit remplir la bonne fiche, geste qui lui permet de créer ou de modifier les produits de formation dont il est responsable.

Le projet G., au moment où nous le décrivons, a fait l'objet d'une fiche de proposition. Il a donc fallu verbaliser l'origine de l'idée, le public visé et le dispositif pédagogique dans ses grandes lignes pour le faire valider par la direction métier. Après cinq mois d'attente, la deuxième étape a été remplie avec la fiche de conception, beaucoup plus détaillée et impliquant la signature de toutes les

\footnotetext{
${ }^{50} \mathrm{~A}$ cet égard, l'introduction de la bureautique et des tableurs pourrait constituer une raison numérique, ou une logique informatique, aussi importante dans ses effets que l'apparition de l'écriture décrite par Goody J. 1979, La raison graphique, Éditions de minuit. Le sens commun. France.
} 
directions métiers qui sont sollicitées pour donner une estimation des ressources (temps et moyens humains) nécessaires pour réaliser le projet. La reprise des supports de cours dans les différentes disciplines (management, finance, ressources humaines, droit, marketing, entrepreneuriat, comptabilité), de la création de services d'accompagnement (suivi de rédaction des mémoires des étudiants, informations trimestrielles sur l'actualité de la gestion, information sur les débouchés après le parcours de formation) des évaluations autocorrectives sur site Internet, des documents d'inscriptions,.... doit être estimée pour obtenir l'accord final du comité de direction, autorisation formelle.

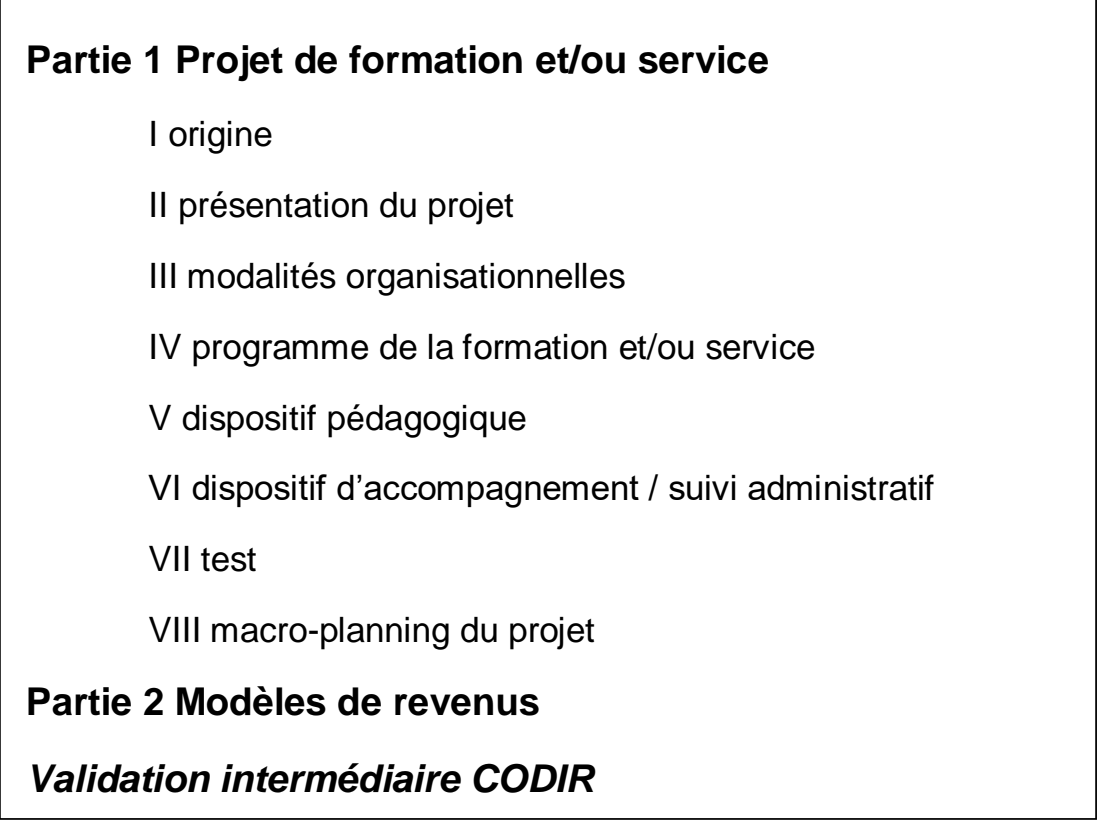

Aperçu de la fiche de conception du nouveau produit de formation (sommaire du document)

Ne nous méprenons pas sur ce délai de cinq mois d'attente entre deux étapes de validation. Plusieurs temps s'enchevêtrent dans la vie quotidienne du responsable de formation : la frénésie du calendrier quotidien (courriels, réunions) ne doit pas être masquée par l'avancée trimestrielle des projets d'ingénierie (comme le projet G.), ni même annuelle des changements de missions et statuts du personnel, trisannuelle des recrutements (la rotation des responsable de formation est de trois ans en moyenne, elle renouvelle la culture d'action commune) et le temps long des réformes (délocalisation du siège dans les années 1990, réforme en directions métiers dans les années 2000). Les grands gestes 
des hauts niveaux se font avec ampleur et plus lentement, ils font longtemps effet. C'est la vision nietzschéenne du grand homme avec ses gestes graves et son déplacement majestueux ${ }^{51}$.

Le temps du projet d'ingénierie est donc variable selon la hauteur de vue que l'on prend. Ceci vaut aussi bien pour les acteurs humains que pour les actants non-humains. Nous opposons ainsi le stylo du responsable de formation qui vibre quotidiennement pour signer les attestations de fin de formation aux lentes rotations des machines de production de cette industrie de la connaissance ${ }^{52}$ : elles nécessitent un temps plus important pour être achetées, installées et allumées ; mais alors elles produisent des milliers de fascicules par jour. Ce sont des actants au geste lent et à l'effet puissant.

Le formulaire à remplir par le responsable de formation peut être vu comme une procédure avec son temps particulier. Le délai de cinq mois n'est cependant pas fixé par la procédure. Il est lié au vivant informel de l'institution : les rapports de pouvoir entre acteurs laissent à moment donné une opportunité pour faire avancer le projet. Le responsable de formation sent en effet un moment opportun car il s'aperçoit que le directeur dont il dépend veut instrumentaliser le projet pour servir ses ambitions personnelles. Le responsable de formation réitère donc sa demande orale et presse ainsi la procédure de validation de la fiche. Une impulsion individuelle du directeur se transforme en décision collective et l'institution avance dans la production d'une formation qui aura des conséquences sociétales. Le projet d'ingénierie de formation prend ainsi de la hauteur : marche à marche, il gravit l'escalier institutionnel que construisent la procédure et ses documents empilés.

\section{Formalités et conformité : l'organisation de l'ingénierie de formation}

Considérons à présent cette procédure et ces fiches comme des formalités. Les formes demandées (une fiche de conception de 17 pages, composée d'une centaine de questions qui sont identiques pour tous les projets d'ingénierie de formation) limitent les gestes du responsable de formation. Il doit répondre aux questions posées, mettre les formes, et non pas présenter librement son projet d'ingénierie.

Ce qui peut paraître une limite du geste s'avère être un amplificateur de l'effet. Car se conformer à la forme commune c'est permettre à l'institution de fonctionner. Les directeurs peuvent comparer les différents projets qui sont semblablement présentés, avec des critères stables, et peuvent décider d'allouer les moyens humains et financiers à tel ou tel projet. La forme est canalisatrice du fonds d'ingénierie : elle conduit et porte le flux d'idées pédagogiques dans le moulin d'administratif qui se met en branle. Sans ces rouages nécessaires la machine ne tournerait pas. À l'échelle du

\footnotetext{
${ }^{51}$ Nous avons soutenu une thèse de doctorat en philosophie sur les valeurs du combat chez Nietzsche.

${ }^{52} \mathrm{Cf}$. Les travaux de Jacques Perriault ou encore Moeglin Pierre, 2010, Les industries éducatives, PUF. Que saisje ? France.
} 
responsable de formation, il faut entrer dans la forme et se conformer (ressembler), à l'échelle de l'institution l'ensemble des formes individuelles se conforment (s'assemblent) en une figure unique.

En termes organisationnel, le responsable de formation doit être organisé, c'est-à-dire accepter de fonctionner comme un organe déterminé. Alors l'organisation peut prendre forme: ensemble d'organes similaires qui fonctionnent en harmonie. Le bon geste du responsable de formation qui remplit les formalités comme il convient et au moment idoine est le signe d'un agent organisé qui permet à l'organisation d'exister. Ce geste est alors amplifié par l'organisation qui lui donne sa force institutionnelle.

La professionnalisation du responsable de formation consiste à apprendre ces bons gestes, à s'identifier à ce travail bien fait pour se fondre dans la culture d'action éducative de l'établissement. Les compétences d'ingénierie de formation sont ainsi pour partie de nature administrative (remplir des formalités) - ce qui permet à l'institution de justifier progressivement le passage du recrutement de personnel enseignant à des personnels gestionnaires plus que pédagogues

\section{Conclusions}

Le projet G. d'ingénierie de formation nous a permis de mobiliser les sciences de l'éducation pour comprendre le fait de gestion éducative. Nous avons maintenu une dialectique du geste et de l'effet pour comprendre cette gestion particulière. Ainsi nous pensons que le responsable de formation débutant gesticule sans effet. Puis il apprend à faire des gestes effectifs et devient novice. Suit l'expérience du confirmé qui fait beaucoup car sa gestion est un ensemble de gestes efficaces. Enfin, l'expert ${ }^{53}$ est efficient, il se situe au niveau de la gestation de projet, de l'ingénierie de formation. Il maîtrise parfaitement les compétences de son métier et effectue beaucoup avec peu de gestes, car ceux-ci prennent les formes idoines et interviennent au moment opportun. Organe adapté à l'organisation, il en améliore même le fonctionnement par ses propositions.

Cette dialectique du geste et de l'effet s'amplifie si elle éclaire les professions de la formation en elles-mêmes: le geste formatif serait celui qui produit un effet formateur dans l'esprit de l'apprenant. Et cet effet produirait lui-même d'autres effets car l'apprenant voit et agit selon ce qu'il a appris. Ainsi l'ingénierie de formation serait acte de gestation à effets gigognes.

\footnotetext{
${ }^{53} \mathrm{Cf}$. les travaux de Bernard Martory sur la gestion des ressources humaines. Martory B., 2010, La gestion des ressources humaines, Dunod. Gestion Sup. France.
} 


\section{Introduction}

\section{Problématique, hypothèse, plan et cadre théorique}

Le projet de ce chapitre est de mobiliser les cadres théoriques français du temps et les concepts de sciences sociales afférents pour analyser l'activité de conception et d'ingénierie de formations d'adultes. Nous prendrons pour exemple le chapitre précédent et le «projet G. » présenté qui constitue un matériau empirique de données ethnographiques.

Notre problématique, ou question de recherche, est « comment analyser l'ingénierie de formation avec la notion de temps, linéaire et cyclique »? Notre hypothèse est que cette notion de temps n'est pas nécessairement celle des sciences de gestion (temps linéiaire et finalisé par une flèche) et notre analyse anthropologique repose sur la description des temps forts puis sur la mesure de leur régularité dans une conception cyclique.

Nous inscrirons ainsi notre analyse dans les réflexions sur l'expérience subjective de l'irréversibilité (Jankélévitch, 2001), sur la mise en récit d'événements à venir ou passés (Ricoeur, 1991) et, dans une perspective de sciences sociales appliquées au fait éducatif, sur les rythmes (Hall, 1992), sur le temps collectif (Mauss, 2002 ; Levi-Strauss, 1987 et Witte, 2010) ou encore sur la mesure et les échelles de temps (Braudel, 2013). Ce cadre théorique restreint à des enseignements contemporains et majoritairement francophones se fonde sur les auteurs classiques en Occident (Heidegger, 1986 ; Parménide, 1998) mais reste au plus près des considérations empiriques modernes.

L'enjeu est d'offrir une description analysée et opératoire des temps de l'ingénierie de formation, utile aux chercheurs du champ pour penser leurs propres objets de sciences de l'éducation. Ainsi, par un travail d'import de concepts philosophiques et de sciences sociales, nous espérons apporter à la communauté scientifique un regard fécond sur les activités de conception de formation.

\section{Description analytique du temps de l'ingénierie de la formation}

Notre approche qualitative nous a permis d'isoler des moments-forts dans la vie d'une organisation de formation et plus particulièrement dans la conception ou l'ingénierie de 
formation. Ces moments-forts reviennent régulièrement et rythment ainsi l'activité, permettant le passage du qualitatif au quantitatif et à une mesure du temps. Notre métrologie passe donc par la définition d'unités de mesure, les moments forts, puis par le décompte de leur scansion rythmée.

\section{Les temps forts : l'intensité du moment}

Concevoir un programme de formation c'est vivre un moment intense où s'entrevoit un parcours avec sa temporalité interne (le nombre de séances de cours, les activités qui s'étalent durant plusieurs jours ou semaines pour les apprenants et le formateur) et une temporalité externe cadrée par l'institution (le temps qu'il faudra pour recruter le formateur, vendre la formation, organiser la logistique du parcours). Cet emboîtement complexe de temporalités devient routine lorsque l'on est employé pour des activités d'ingénierie de formation : chaque jour amène ses nouveaux projets de formations, ses nouvelles idées de programmes et leur réalisation à mettre en œuvre - et une partie seulement verra le jour. Le chargé d'ingénierie de formation travaille sur plusieurs projets en parallèle, il est donc confronté à une grande diversité de temporalités qui s'enchevêtrent.

Le quotidien de l'ingénierie de formation est fait d'une veille sur les tendances d'un champ de formation particulier. Nous avons par exemple été abonnés aux différents journaux de la formation professionnelle pour nous informer de l'actualité de leurs besoins de formation. Cette lecture routinière n'est pas lisse : elle présente des temps forts de lecture, au moment où un sujet proche de sa formation attire notre attention et concentre nos efforts. Parfois, au détour d'une phrase ou d'une offre concurrente, après un moment de réflexion personnelle, une idée de formation émerge. Ce moment de conception implique une activité mentale intense et en quelques dizaines de secondes des informations recueillies durant plusieurs jours ou semaines sont croisées pour donner naissance à une forme unique. Le temps se condense pour l'esprit qui est absorbé par sa réflexion, avant d'en être libéré sous la forme d'un résultat (un énoncé du thème formation, une image du public visé, peut-être quelques intuitions sur le programme). Une fois ce moment de conception créatif passé, le chargé d'ingénierie de formation peut retourner à des activités plus routinières où son effort mental se dissipe : lectures distraites, pauses avec les collègues pour soumettre son idée (ce qui permet de le libérer de son état d'excitation et d'avoir un avis extérieur), tâches moins complexes comme la planification ou les réalisations diverses, etc. 
Le moment intense, ou moment-fort, peut aussi être collectif (Mauss, 2002). Ainsi une réunion est un fait social qui totalise la vie d'une équipe ou d'un service administratif. Les différents collègues se réunissent autour d'un thème pour en débattre. Ce peut être une idée de formation précise ou, plus génériquement, une modalité pédagogique à adopter par tous. Ainsi le format ECTS peut être débattu pour mesurer la valeur des formations à distance. Ou encore l'importance des méthodes actives à intégrer dans les formations professionnelles continues. Ce moment-fort où chacun peut exprimer son point de vue, débattre et se comparer aux autres est un lieu important. Nombre de pratiques collectives s'y cristallisent, comme la bonne temporalité. Ainsi les plus anciens dans le métier peuvent conseiller les novices sur le temps qu'il faut prévoir pour recruter tel ou tel auteur, sur la durée de la procédure de contractualisation avec les services administratifs ou juridiques. De même, le groupe de chargés d'ingénierie en son ensemble peut être dépendant de la temporalité d'autres services : le service de production qui imprime les supports de formation et diffuse les contenus sur les sites web peut profiter de ce moment collectif pour rappeler son propre calendrier de travail et contraindre l'activité de conception par les impératifs techniques

Durant réunion de service des chargés d'ingénierie de formation le temps passé ensemble est un moment-fort. C'est un moment qui condense le temps : plus d'informations sont échangées, chacun s'ajuste rapidement aux autres. Les périodes de travail en solitaire qui précèdent ou succèdent à ces moments collectifs intenses paraissent être des périodes de repos, ou d'activité moindre. La réunion n'est cependant que rarement le moment où une nouvelle idée de formation est conçue : c'est plus souvent une confrontation aux pratiques et idées des autres qui permettent de la développer. Soit parce que des modalités pédagogiques de service doivent la compléter, des contraintes d'un autre service la déterminent, soit parce que le responsable hiérarchique pose des questions qui amènent des développements ou encore parce qu'il évalue et synthétise les données. Le simple fait d'avoir à présenter le projet d'ingénierie de formation demande de la préciser et de la détailler. Cette clarification du projet peut passer par la production d'un calendrier ou d'une frise listant les principaux moments du développement de la formation imaginée. Autrui intervient comme un miroir réfléchissant et déformant, qui demande de s'exposer avec son projet. Il créé un temps commun durant lequel les idées sont partagées et développées.

Le temps fort, moment intense de conception ou moment social de coopération, vient en amont de la formation, dans sa prévision. Une fois la formation acceptée par l'institution, vient alors le temps de la réalisation, qui lui aussi connaît ses temps forts. Les dates importantes sont liées à la temporalité commerciale de la vente du produit de formation en 
organisme de formation : le jour de la mise sur catalogue, la signature par les commerciaux d'un contrat avec un client important déclenchant plusieurs inscriptions, le jour où le nombre d'inscrits à la formation dépasse le nombre minimal viable et permet son maintien prévisionnel, etc. Cette temporalité des inscriptions est faite d'informations venant régulièrement des services ou bases de données concernées, des interactions avec les commerciaux ou les webmasters. Ce sont des moments marqués par l'attente et le relâchement subit de l'anxiété lorsque la bonne nouvelle est annoncée : instant d'assurance confirmée lorsque les inscrits entrent dans le cadre du projet de formation, instants d'agacement, de doute, voire de panique, lorsqu'il manque une information sur le site Internet ou lorsque la formation est annulée. La prise en compte de l'affect (Barbier, 1998) et des émotions est donc primordiale pour comprendre les temps fort de l'ingénierie formation et leur vécu.

Dans l'ingénierie de formation à distance : certes les moments-forts sont moins liés à des présences en salle que pour la formation professionnelle continue (quoique les formations hybrides le permettent), mais ils s'articulent également autour des inscriptions, des dates d'ouvertures des sites Internet ou d'envoi des colis de cours papier. La tension entre les partenaires mobilisés (éditeurs tiers, écoles partenaires, etc.) peut intensifier des moments comme la remise d'un stock d'ouvrages à distribuer ou le changement de plateforme numérique de formation. Dans ce dernier cas, un nouvel environnement numérique de travail demande quelques jours d'adaptation pour en comprendre ou retrouver les fonctionnalités. Ce moment-fort de découverte et de prise en main relève de la construction du sujet en interaction avec son environnement (Barbier, 2006). Le moment-fort n'est plus ici dans l'interaction entre sujets, dans un temps collectif, mais bien dans l'interactivité avec la machine ou un environnement d'objets - quoique l'usage industrialisé, à grande échelle, d'un outil de formation, puisse faire intervenir un temps fort collectif d'interactions avec l'outil : un ensemble de concepteurs de formation travaille à comprendre le maniement du nouvel outil dont ils feront chacun un usage individuel. Ici le temps collectif prépare et encadre les temps individuels qui succèderont.

L'ingénierie de formation à distance, dans l'institution observée, est complétée par un suivi des formations existantes: le chargé d'ingénierie de formation doit répondre de formations, c'est-à-dire à la fois soutenir leur viabilité économique, leur pertinence pédagogique et leur adaptation technique à l'état de l'art lorsque sa hiérarchie la met en question. Il assure dans ses missions la qualité pédagogique et est donc amené à effectuer des 
mesures : comptabilité des coûts, calcul du chiffre d'affaires et de la rentabilité ; taux de retour des copies et satisfaction des corrections ; veille sur les outils informatiques du moment utilisés par les concurrents et intégration des préconisations de l'institution. Chacune de ces mesures est faite en réponse à une demande de la ligne hiérarchique et déclenche un pic d'activité pour récolter les données et les formaliser. Le moment-fort initial est autour de la bonne compréhension des questions posées et la procédure pour trouver dans ses formations la réponse juste. Puis un moment notoire est une mesure négative où le responsable de formation voit que tel indicateur n'est pas satisfaisant et appelle une réingénierie de la formation. Après un moment de déception intense, il remet alors sa formation sur le métier de l'ingénierie pour l'améliorer vers des standards acceptables - ou il la supprime si elle n'est pas rentable, pas pédagogiquement ou techniquement adaptée.

La description de ces moments-forts dans le quotidien d'un concepteur de formation que ce soit en organisme de formation professionnelle continue ou dans la formation à distance - relève d'une approche discontinue du temps. Chaque moment semble isolé et indépendant l'un de l'autre. L'intensité émotionnelle ou affective du moment, le degré de fertilité et de condensation du temps, pourraient peut-être se mesurer - encore faudrait-il donner des indicateurs psychologiques, pédagogiques... Mais nous préférons prendre une autre voie en contemplant les régularités et les retours périodiques de ces moments-forts. Le passage au quantitatif se fait ainsi par une approche continue du temps, sous forme de cycles revenant régulièrement et pouvant être décrits et mesurés.

\section{De temps en temps : rythmes d'une activité de conception}

Les moments-forts que nous avons décrits qualitativement peuvent être classés en intensité psychologique, intensité sociale, ou intensité d'interaction avec l'environnement technique. C'est un des patrons temporels que nous cherchions à faire émerger de la diversité des lignes de temps et des moments ponctuels. Pourtant nous voulons privilégier une autre approche : en observant les retours cycliques de ces moments-forts, nous pouvons, comme le font les historiens (Braudel, 2013) distinguer des périodes de plus ou moins grandes amplitude, des temps courts et des «temps longs », quoique plus modestement à l'échelle de notre observation ethnographique qui s'incsrit dans une histoire brève.

Le temps d'une réflexion débouchant sur l'ébauche de conception d'une nouvelle formation est un temps court - limité à quelques minutes - et très intense (tension de l'esprit, 
fécondité du moment qui cadre les développements suivants). Cependant, c'est un «temps long » au sens des historiens car il se manifeste en permanence et constitue le cœur de l'activité. À l'échelle du métier, il ne constitue pas une période déterminée qui ne serait qu'un bref passage transitoire : c'est l'essentiel de ce qui est demandé. Tout au plus pouvons-nous constater un étirement de ce temps dans le sens où les projets de formation deviennent plus complexes avec l'ancienneté et le métier : plus d'éléments sont à prendre et compte et des programmes plus complets et souvent plus longs sont conçus. Le jeune chef de projet en organisme de formation professionnelle réfléchit à un programme de trois jours, puis à la conception de stages répartis sur une année, puis il pense une section de son catalogue de formations, un thème se déclinant en une série de formations brèves. De même, le chargé d'ingénierie de formation commence par prendre en main des formations existantes d'une durée de une à deux années, à améliorer le dispositif par des mises à jour ou des parties du programme repensées, puis il s'ingénie à créer des projets mobilisant plusieurs institutions (universités, éditeurs de livres, éditeurs de logiciels, etc.), concernant des centaines d'apprenants sur plusieurs années. Le temps contenu dans l'ingénierie s'étire, il est de plus en plus long, mais l'activité d'ingénierie reste constante. C'est à la fois un des temps les plus courts lorsqu'il est chronométré (des activités de réflexions de dix minutes), le plus fréquent et le plus long dans le sens où il est quasiment immuable et où son contenu s'étire.

Dans ce « longtemps » du temps long historique, voire ce « toujours » qui fait le quotidien de tous les jours, s'encastrent des temps plus courts et moins fondamentaux qui sont les temps sociaux de co-ingénierie. Paradoxalement, le temps social peut paraître plus long dans le sens où une réunion, un moment collectif, dure souvent plus d'une d'heure. Encore faudrait-il subdiviser les interactions, dégager les moments forts où tout le collectif est véritablement mobilisé (attention commune et fort échange d'informations, vote, prise de décision sur un sujet sensible), des moments faibles où le groupe se met en place, débat en aparté, attend patiemment la fin d'un soliloque, etc. Cette étude des temps sociaux, leur décomposition et leur mesure a été détaillée par E.T. Hall (1992). Nous sommes cependant plus intéressés par le retour des temps, leur fréquence plus ou moins haute, et non le détail d'un période. Le temps long des historiens consiste en effet non pas en la durée d'une période mais dans la durée pendant laquelle cette période revient fréquemment. C'est une question d'échelle qui se mesure en nombre d'années d'exercice à l'échelle du métier et non en minutes ou heures du moment. 
Le temps social est un temps plus court dans le sens où c'est un temps qui intervient différemment selon l'ancienneté. La socialisation à ce métier passe en effet par une période de test où le concepteur, certes encadré par un autre employé qui le guide, est relativement seul pour faire face aux contraintes du métier. Il sollicite autrui pour comprendre le fonctionnement de l'organisation et ses outils. Mais il doit faire ses preuves en passant l'épreuve des premières conceptions et réalisations. Il doit montrer seul qu'il est bien adapté, c'est-à-dire à la fois que son expérience du métier est positive (il ne part pas ou ne cherche pas un autre poste) et que les résultats attendus sont satisfaits (il est utile à l'organisation). Après ce premier temps court d'ingénierie solitaire en période d'essai (qui peut être la période légale ou durer d'un mois à un an, le temps de gagner la confiance de ses collègues), vient une deuxième étape où le jeune employé intervient dans les moments collectifs mais à titre mineur. Il participe à la co-ingénieirie en réunion mais modestement, en complétant le propos de ses aînés ou en donnant un avis souvent peu pris en compte. Cette période dure jusqu'à ce que le concepteur de formation ou le chargé d'ingénierie gravisse l'échelle formelle ou informelle de son environnement de travail. Soit que son succès est reconnu et qu'il obtienne une position hiérarchique officielle qui le légitime, soit que ses collègues qui le connaissent bien apprécient son avis sur tel ou tel point précis pour lequel ils le savent compétent. Ce troisième temps social, de chefferie ou d'expertise, est le plus long - tout au moins l'organisation a-t-elle intérêt à maximiser ce temps où l'employé, très sollicité par ses collègues, est utile. Le concepteur peut, lui, choisir entre le confort d'un poste où il est reconnu, ou avoir l'ambition de valoriser cette expérience réussie en continuant son parcours dans l'organisation ou en sollicitant d'autres organisations. L'activité sociale est ainsi de nature changeante et se décompose en trois temps plus courts que le temps long ou permanent de l'activité psychique de conception. Cette activité sociale, faite de réunions qui reviennent à rythmes réguliers fait appel à des temporalités multiples : entretiens hebdomadaires avec le responsable hiérarchique au début, puis semestriel, voir annuel une fois l'autonomie acquise. Mais on assiste, en cas de promotion ou de changement de poste, à un retour à des réunions plus fréquentes avec un nouveau supérieur hiérarchique .... La co-ingénierie change d'ampleur mais reste la constante. De même, la participation à des réunions de service, au début peu fréquentes, se multiplient et reviennent hebdomadairement. Et le concepteur y prend une part de plus en plus centrale au point que cela constitue le cœur de son activité : il participe à l'ingénierie collective et son avis pèse sur les décisions des uns et des autres. Là où il suivait le rythme collectif, il bat de plus en plus souvent la mesure pour donner la cadence et imposer ses visions du temps. 
D’après nos données empiriques, il semble que le temps social de conception collective dépende de la taille de l'organisation. En effet, une grande institution administrative fait coopérer des métiers très différents et spécialisés, ne parlant pas le même langage, et présente une hiérarchie telle que les procédures, souvent formalisées avec fiches et signatures des bons interlocuteurs, ralentissent les prises de décisions. Obtenir le budget et l'autorisation de mettre en œuvre un projet peut prendre plusieurs mois, voire quelques années. À l'inverse, dans une petite entreprise à l'organisation changeante (Marty, 2002), les temps sont plus brefs, les décisions plus rapides car moins d'interlocuteurs spécialisés et hiérarchisés sont sollicités. La mise en œuvre de la conception est elle aussi dans des temporalités différentes : la grande institution s'engage sur des programmes longs et utilise une grande variété d'objets complexes (environnements virtuels, outils numériques ou d'impression papier, partenariats inter-institutionnels...) qui demandent une inertie lors de leur mise en route. À l'inverse, une petite société spécialisée dans un secteur précis décide rapidement de mettre en œuvre l'ingénierie de formation qui s'appuie sur des objets plus simples (location de salle, catalogue papier) ou moins variés.

Plusieurs temps s'enchevêtrent ainsi dans la vie quotidienne du chargé d'ingénierie formation, comme l'écrirait l'anthropologue E..T Hall dans La danse de la vie : la frénésie du calendrier quotidien (courriels, réunions) ne doit pas être masquée par l'avancée trimestrielle des projets d'ingénierie, ni même annuelle des changements de missions et statuts du personnel, trisannuelle des recrutements (la rotation des chargé d'ingénierie de formation est de trois ans en moyenne, elle renouvelle la culture d'action commune) et le temps long des réformes (délocalisation du siège dans les années 1990, réforme en directions métiers dans les années 2000). Les grands gestes des hauts niveaux se font avec ampleur et plus lentement, ils font longtemps effet. C'est la vision nietzschéenne du grand homme avec ses gestes graves et son déplacement majestueux.

Le temps du projet d'ingénierie est donc variable selon la hauteur de vue que l'on prend. Ceci vaut aussi bien pour les acteurs humains que pour les actants non-humains. Nous opposons ainsi le stylo du chargé d'ingénierie de formation qui vibre quotidiennement pour signer les attestations de fin de formation aux lentes rotations des machines de production de cette industrie de la connaissance (Moeglin, 2010) : elles nécessitent un temps plus important pour être achetées, installées et allumées; mais alors elles produisent des milliers de fascicules par jour. Ce sont des actants au geste lent et à l'effet puissant. 
Nous touchons ainsi au le troisième type de moment fort, celui qui concerne les interactions avec l'environnement technique, qui fait l'objet de cycles plus variés encore. Les cadres temporels techniques sont marqués par des temps courts dans le sens où les avancées techniques rendent rapidement obsolète telle ou telle outil. Ainsi la temporalité imposée par la poste papier représente un temps passé dans l'ingénierie de formation à distance qui se fait aujourd'hui autour de la numérisation des documents. Et avant cela, plusieurs temps se sont succédés et sont parfois encore utilisés : téléphone, télévision, minitel, mél, site Internet, classe virtuelle. Les environnements virtuels numériques d'apprentissage permettent d'intégrer ces objets multiples aux temporalités variées : il faut ainsi concevoir des temps de lecture (variables selon les lecteurs), des temps de visionnage de vidéo (constants quel que soit le lecteur), des corrections instantanées car automatisées de question à choix multiples, des temps de correction de copies abrégés par une transmission électronique... L'outil moderne tend vers le raccourcissement des délais, voire l'instantané, et le foisonnement des supports de formation avec chacun leur temporalité propre. L'interaction entre enseignants et enseignés est multipliées et peut prendre plusieurs canaux de communication, chaque apprenant pouvant choisir sa temporalité et son mode de communication. Le concepteur de la formation doit tenir compte de tous ces outils avec leurs temporalités variées et anticiper les utilisations différenciées par les utilisateurs - apprenants d'un côté, enseignants à distance de l'autre qui doivent savoir répondre dans les temps normés par l'institution (délais de réponse) et adaptés à l'outil.

Le chargé d'ingénierie de formation dispose d'un outil de mesure comptable des coûts d'une formation. Cette outil, dans l'institution observée, prend la formation d'une feuille de calcul dans un tableur où tous les postes de dépenses sont listés - la feuille de calcul se chargeant de les additionner pour donner un coût total et le comparer au chiffre d'affaire pour effectuer une analyse financière de la marge et de la rentabilité. L'institution oblige ainsi le chargé d'ingénierie à évaluer les coûts en comptant le nombre de jour de travail à tous les postes : il doit, en concertation avec les responsables des services concernés, estimer combien de jours sont nécessaires à la production d'un nouveau site Internet, combien de minutes prennent une inscription pédagogique d'un apprenant, combien de temps nécessite la mise en page d'un document. L'anticipation du temps de contractualisation administrative d'un auteur vacataire, ou encore des délais de livraison des cours, ne font pas partie de cet outil. Mais ce sont toutefois des estimations temporelles qui sont demandées par l'institution et font partie du métier d'ingénierie : la directive est d'optimiser les rapports coûts/délais/qualité. Là où le 
chargé d'ingénierie semble très libre dans l'organisation de son travail - aucune tâche chronométrée ne lui est demandée - il doit contrôler le temps et en mesurer la valeur : c'est une partie primordiale de son métier.

Lors de la mesure des temps de travail pour calculer les coûts d'une formation avec l'outil de bureautique, le chargé d'ingénierie indique aussi l'espérance de vie de la formation. Le nombre d'inscription peut ainsi varier d'une année à l'autre durant le cycle de vie de la formation et la rentabilité s'en trouver affecter. Cette mise en perspective des coûts et des revenus fait l'objet d'un deuxième outil : une série de fichiers de texteur décrivant le « cycle de vie de l'offre ». La formation est pensée comme un organisme vivant ayant son espérance de vie. Chaque étape du développement du projet d'ingénierie de formation est marquée par un document qui doit être validé par le comité de direction idoine. Le produit de formation a ainsi un cycle de vie qui débute avec une fiche de proposition de nouvelle formation, suivie d'une fiche de conception de la nouvelle formation (impliquant les différents métiers de l'institution : production, commercialisation, scolarité, innovation) et ainsi jusqu'à une fiche de suppression de la formation. Le chargé d'ingénierie de formation doit remplir la bonne fiche, geste qui lui permet de créer ou de modifier les produits de formation dont il est responsable.

Comme le décrit Bruno Latour dans son œuvre de sociologie des sciences et des techniques, des actants divers sont impliqués : les acteurs «humains » avec leurs temporalités propres, leurs routines et leurs rythmes personnels, sont confrontés à des acteurs «nonhumains », des machines et des objets eux aussi impliquent un temps particulier. Il faut le temps de voir, de prévoir, de revoir pour faire fonctionner ces outils dans les temps qui leurs sont propres.

L'analyse des rythmes des temps psychiques de conception individuelle, sociaux de co-ingénieirie et techniques de manipulation d'objets pour réaliser la formation, nous amènent à un motif unique autour de trois lignes de temps. Chaque temporalité a une amplitude (la taille des points), une fréquence (le nombre de leurs retours sur la flèche du temps) et éventuellement une période (ainsi les temps sociaux sont divisés en trois périodes). La description des moments-forts s'ordonne ainsi en un tableau des rythmes cohérents : 
Temps de l'activité psychique de conception : un temps long ou permanent

Temps sociaux de co-ingénieirie : trois temps courts distincts

Temps des objets à manipuler : diversité selon les techniques

\section{Le patron des temps d'ingénierie de formation}

Après avoir décrit les principaux moments-forts de la conception, puis vu comment ces moments revenaient en rythmes enchevêtrés, dépendant de leur mode psychique, sociale ou physique, voyons comment le concepteur peut produire un discours sur ces temps-là. Comment il peut, pour reprendre une des perspectives de Paul Ricoeur (1991), mettre le temps en récit.

\section{Conclusion : le temps en récit}

Notre description analytique du temps de la formation nous a permis de répondre à notre question de recherche en validant notre hypothèse : le temps de l'ingénierie de la formation se mesure bien par une double étude des temps forts et de leurs rythmes. Ajoutons à cela des remarques épistémologiques qui vont servir d'ouverture à notre conclusion.

Produire un discours sur le temps passe par une distanciation avec le temps vécu et son esthétique propre pour passer à une narration qui elle-même a sa propre temporalité : le temps de l'ingénierie de la formation.

\section{Vivre le temps qui passe}

L'esthétique du temps, son ressenti au fil des terrains d'observation, relève du vécu subjectif du praticien et de l'expérience quotidienne sur les lieux d'enquête. Vladimir Jankélévitch (2011), dans ses écrits fondamentaux sur la perception du temps, indique la 
notion d'écoulement du flux temporel et l'irréversibilité de ce qui ne peut plus revenir. Le temps qui passe est déjà passé, et la surprise est d'autant plus grande lorsque le bon temps, invisible et indolore, fait l'objet d'une rétrospection analytique. La succession des moments forts, lorsque vécus plaisamment, appelle une nostalgie impossible : ce qui a été ne peut plus être à nouveau et le retour en arrière est illusoire. Le temps qui s'est écoulé devient soudain visible : il est revu à défaut d'avoir été vu dans une attente impatiente. Les moments de détente ou de frénésie qui en ont fait l'écoulement variés sont saisis, les sensations vécues sont alors rappelées. Tout n'est pas noté sur les carnets de terrain et l'effort de mémorisation guidé par l'écriture finale fait ressurgir des faits trop importants ou trop minimes pour avoir été notés au fil du temps d'observation

Un des biais possiblse de notre enquête réside dans cette subjectivité du temps vécu : les moments-forts que nous avons tenté d'objectiver sont liés à notre expérience et notre ressenti. Il serait ainsi possible d'en oublier certains, faute de les avoir senti passer, voire d'en exagérer d'autres qui plaisaient ou déplaisaient à un moment notoire. Toutefois, le recours à des discussions avec des collègues indigènes, l'objectivation dans des procédures (fiches de conception décrites, fiches du calcul de la valeur basée sur le temps de travail,...) et la comparaison entre deux organisations permet de relativiser cette part de subjectivité. L'intersubjectivité et les objets viennent conforter notre choix des moments-forts.

Comme le remarquait Maurice Halbwachs (1994) au début du 20 ème siècle, dans une étude devenue classique récemment, la mémoire est le produit d'une activité collective. Le choix de retenir tel ou te moment-fort est dépendant du cadre épistémique du laboratoire : la vision ou la mémoire des faits est cadrée d'une part par la mobilisation des affects et des émotions, c'est-à-dire du psychique; d'autre part par l'insistance sur le collectif et l'interaction, c'est-à-dire le social. Le thème du métier, de l'outil technique de travail, mais aussi de l'art et des compétences qu'il faut pour l'exercer, est le cadre de l'institution Cnam dans lequel s'insère le centre de recherche sur la formation. Le travail cognitif de recherche, et de mémoire en particulier, est donc déterminé par un jeu d'emboîtements épistémiques. Et à ce cadre-présent vient s'ajouter les cadre-passés de notre formation de chercheur en sciences sociales : l'attention aux règles et pratiques d'organisations dérive de cadres sociaux de la mémoire passés et pourtant toujours vivaces.

L'exercice de mémoire du chercheur est d'autant plus difficile qu'il est contradictoire avec les pratiques cognitives du métier d'ingénierie de formation. Comme nous avons tenté de le montrer, le concepteur de formation est tourné vers l'avenir et non vers le passé. C'est une culture de travail qui relève de la prévision plus que de la révision (Witte, 2010), de la 
prospection et rarement de la rétrospection. Il faut prévoir des formations, anticiper les problèmes, planifier la réalisation. Rares sont les regards sur ce qui a été - autre que pour améliorer ce qui sera. Le référentiel est donc « demain », « hier » n'étant vu que comme une préparation continuée «aujourd'hui » dans cet objectif. À l'inverse, le regard rétrospectif de l'analyse et de la narration, sort de ce cadre cognitif d'une profession de la prospection.

\section{Ecrire le temps passé}

Nous avons noté que notre exercice de mémoire s'inscrit dans les cadres d'un laboratoire de recherche. Au-delà de l'épistémè, des auteurs mobilisés et de l'inscription disciplinaire, la temporalité propre du laboratoire avec ses exigences de production et de résultats de recherche, détermine l'activité rétrospective et narrative. C'est le cadre social du laboratoire qui met en travail réflexif la mémoire du praticien : il recherche alors dans ses notes et documents, dans ses souvenirs, les éléments pour produire le discours nécessaire dans les temps qui lui sont imposés. L'expression de la recherche est circonstanciée, fruit des pressions et opportunités conjoncturelles, qui déterminent une mise ne forme

Cet exercice d'écriture, où il faut décrire le temps de l'ingénierie, repose sur des moments distincts : d'abord le recueil ethnographique d'informations - vécu, observations, entretiens, notes ; l'observateur est alors sujet du temps auquel il est soumis. Puis le temps de la rumination, digestion et gestation d'une analyse selon les cadres du laboratoire et dans les temps demandés : c'est là que le participant prend de la hauteur et fait du temps un objet qu'il peut observer et jeter devant lui. L'objectivation du temps passe donc par une prise d'altitude pour voir l'ensemble des moments et des rythmes. Cette objectivation ne se fait pas d'un seul coup mais au fil des temps de réflexion. Dans notre cas, les principaux moments de cette réflexion sont présentés par les documents successifs sur https://cv.archives-ouvertes.fr/marty.

À l'exercice d'écriture répond l'exercice de lecture : Paul Ricoeur a montré comment les déformations du temps sont majeures lors de cette ultime phase de la mise en récit. Le temps réel de l'action est déformé par le temps du récit, qui accélère ou ralentit selon le narrateur, sans prendre en compte le déroulé concret des événements passés. Et le temps du lecteur, celui du parcourt dans l'œuvre, passant furtivement sur tel paragraphe, s'attardant sur tel autre, est encore un autre temps avec sa logique propre qui s'éloigne des archives de la physique. Les faits passés sont présentés avec un temps, lus dans un autre, et l'histoire en son 
entier ne retient plus que quelques événements qu'elle à peine à chronométrer ; voire quelques dates difficiles à hiérarchiser.

La physique et les forces de la nature prennent toutefois leur revanche sur l'histoire des narrations humaines en imposant des objets qui cadrent les temps de lecture et d'écriture. Les temps institutionnels se manifestent dans des contrats de recherche qui déclenchant le versement de ressources matérielles, ou encore dans des rythmes de publication objectivés par des calendriers marqués de dates butoirs. La gestion du temps devient alors un élément central de la performance - depuis la conception jusqu'à la mise en récit. 


\section{Les métiers de l’ingénierie de formation : variations sur les ingénus}

Le propos ethnographique vise «les ingénus » au centre des enseignements à distance, ici ces ingénieurs de formation que l'on a observés et dont on a fait partie intégrante durant plusieurs années. Le dictionnaire Littré donne plusieurs acceptions au terme : est ingénu celui qui est libre, non asservi. Il peut donc montrer ses sentiments et s'affirmer aisément. En désignant ainsi ceux que l'on a étudiés on donne à voir les conditions de l'enquête (les ingénieurs se sont livrés sans inhibition et se sont prêtés au jeu de la description par le chercheur) comme la différence entre le chercheur et l'ethnie concernée. En effet, le chercheur, loin d'être un ingénu inféodé, était doublement asservi à son travail de recherche en plus de son travail dans le fief de l'institution étudiée. Il devait par là-même souvent masquer ses intentions et ses sentiments du fait de cette vassalité voulue et besogneuse. Les ingénus ne sont donc ni du genre du Huron décrit par Voltaire, ni assimilés aux romantiques de Verlaine, mais une ethnie à l'éthique bien réelle mêlant des caractéristiques des deux : des hommes et des femmes issus de l'enseignement pour administrer des programmes d'agrégation du secondaire, avec leur liberté d'expression et de sentiment face au chercheur réduit au silence.

Miroir laborieux non pas de tous les ingénieurs mais d'une nouvelle forme d'ingénierie (l'ingénierie de formation), nous nous sommes efforcés d'en décrire les engins propres (plateformes numériques, outils de diffusion des connaissances du livre au didacticiel en passant par le téléphone) et le génie afférent (que nous qualifions de génie social, relationnel ou pédagogique, voire parfois gestionnaire du fait de notre entrée dans le champ). Les ingénieurs correspondants, travailleurs de l'enseignement à distance, ne sont donc pas des ingénieurs absents, car on cherche parfois à excuser ce type d'enseignement qui ne vaudrait que pour ceux qui ne peuvent être présents à la formation. Leur ingéniosité est celle d'une présence différente, assurant une permanence pédagogique, affective et sociale, comme l'écrit Annie Jézégou, sur des dispositifs médiatisés qui peuvent être mobiles ou encore hybrides.

Nous commencerons ainsi par décrire le génie coopératif, ou de correspondance, de ceux qui travaillent à des activités de conception en organisation éducative assurant l'enseignement à distance. On verra dans le premier chapitre un travail d'ingénierie collective qui montre la compétence relationnelle. Ainsi la mise en place d'une soutenance collective de mémoire par classe virtuelle sera l'occasion d'élaborer un modèle algébrique (ou façon de 
réunir la classe) et de réfléchir aux conditions de mémoire collective, de cadre matériel et de stratégies individuelles qui déterminent le fonctionnement d'un groupe dans le temps. Notre ethnologie de l'ingénierie pédagogique et l'ingénierie de formation continuera ainsi la réflexion de la section précédente sur le temps en y ajoutant la dimension collective dans le jeu d'acteurs des ingénieurs étudiés et en insistant sur la valeur présente du moment et ses constructions circonstanciées de futurs et passés. Ce travail sur le génie pédagogique sera enfin l'occasion, dans le même chapitre, d'une réflexion sur les conditions de passage d'une ethnologie simple et fondée sur une éthique, à une anthropologie comparant plusieurs terrains ou métiers, voire aires culturelles dans les métiers de l'enseignement.

Le second chapitre est la charnière entre la compétence professionnelle et l'outil de travail, entre le génie et l'engin. À l'art de la coopération pour la conception de dispositifs de formation est associé un métier, ou machine, multimédia. Le savoir-faire se matérialise et est issu d'un dispositif technique. Nous distinguerons deux types d'outils utilisés sur le terrain : d'une part les savoirs médiatisés et d'autre part les intermédiaires éducatifs. Cette distinction permettra alors d'analyser les arts et métiers de l'organisation sur site, c'est-à-dire les compétences professionnelles de ceux qui manient ces deux types d'outils. La réunion de ces arts complémentaires en une organisation industrielle unifiée montre la valeur productive de ces métiers.

Nous évoluerons ainsi vers un chapitre dédié aux engins de formation, aux machines et outils que ces sont les théâtres numériques. Ceci sera l'occasion d'un effort sur les appelations des plateformes d'enseignement en ligne, laissant à la vision une place importante (classe virtuelle avec sa caméra, environnement informatique pour l'apprentissage humain avec ses icônes et tableaux de bord), et permettra de réfléchir aux mots-clés de l'enseignement, qui fondent ses réalités. Ainsi on s'appuiera sur l'histoire des engins pédagogiques et de leurs appelations depuis la télégraphie jusqu'aux cours en lignes ouverts et massifs (Moocs) - en réfléchissant au vocabulaire employé pour les désignér les machines à apprendre selon Eric Bruillard et Georges Louis Baron, et à ses implications quant au sens des activités.

Enfin, notre dernier chapitre présente un projet d'ingénierie de formation que nous avons mené et qui s'apparente à de la recherche finalisée et orientée vers la production de résultats (création d'un diplôme par le ministère). Ainsi la frontière entre la technique (ingénierie de formation) et la science (de l'éducation) est montrée comme ténue du fait des similitudes dans l'action. De même, l'identité de l'ethnographe du corps technique de l'enseignement est ambivalente du fait de sa double appartenance à deux mondes proches et 
en collaboration permanente, qui l'amènent à œuvrer sur les deux terrains qui parfois se confondent. Au-delà de cette question de méthode, l'intérêt de la présentation de ce court projet est l'insertion de l'ingénierie de formation à distance dans son contexte international et dans l'évolution de l'enseignement à distance en France. Enjambant le fossé peu profond entre science et technique, on complètera ainsi nos descriptions ethnographiques de l'ingénierie comme production avec ses actes quotidiens, par la description d'un produit relativement finalisé (un dispositif conçu) - nous invitons à compléter la lecture de ceci par l'expérience apprenante dans un dispositif à distance, pour les plus assidus. 


\section{Introduction}

Nous voulons interroger un verbe d'action (opérer), à dimension collective (comme l'indique le préfixe co-) - ce qui implique, comme nous le verrons, un temps et un espace commun à un groupe d'acteurs, une organisation et des instruments que nous allons questionner. Ce chapitre se veut être une modélisation scientifique, écrite depuis un poste à l'université, d'une expérience professionnelle dans l'enseignement à distance tenant lieu de donnée empirique. Tirant parti du binôme identitaire «enseignant-chercheur », nous voulons étudier avec les cadres analytiques du chercheur les fonctions professionnelles de l'enseignant.

Nous partons donc de données empiriques récoltées par l'observation participante durant un terrain de trois années (2011-2014) dans une industrie éducative de l'enseignement à distance. Ce terrain nous a amené à organiser une ingénierie didactique centrée sur une évaluation. Il s'agissait de répondre à la demande de la direction générale par un dispositif d'évaluation d'un mémoire collectif par soutenance lors d'une classe virtuelle. L'idée était que le groupe de trois étudiants s'étant affairé à un mémoire commun durant une année devait le présenter et le défendre collectivement devant le corps professoral au cours d'une soutenance à distance, par classe virtuelle.

L'ingénierie didactique demandée rentrait dans les cadres administratifs de la commande et est passée par une phase d'ingénierie de formation consistant à mesurer la rentabilité économique du projet (par le biais d'une matrice des coûts et recettes fournies par l'administration sous forme de tableur) et à mieux cibler les étudiants amenés à être évalués de la sorte (l'évaluation s'inscrivait dans un programme destiné à être vendu à des écoles de commerce pour leurs étudiants, ce qui impliquait la rencontre des directeurs d'études de ces établissements). Cette ingénierie de formation entrant dans une logique de gestionnaire s'est déroulée en parallèle de l'ingénierie pédagogique, centrée sur les modalités de l'évaluation innovante. Il a ainsi fallu retenir l'outil de classe virtuelle (qui relevait d'un accord entre l'administration et un fournisseur), et choisir les fonctions (partage d'application et de navigation) qui pourraient être utilisées au cours de la soutenance collective. Un calendrier du mémoire collectif à réaliser à distance par le groupe d'étudiant durant une année a été élaboré 
et devait permettre d'arriver à l'évaluation finale par soutenance avec un important matériau à présenter et défendre.

La capture d'écran ci-dessous présente une classe virtuelle : avec la photographie du locuteur principal et le nom des autres participants figurant au-dessous. Le document partagé «General Physics » apparaît sur l'écran de tous les participants qui peuvent donc le commenter en parlant au microphone et être entendus de tous. Dans le cas de la soutenance collective, les participants sont les étudiants et le correcteur, le document partagé présente le mémoire à défendre durant l'évaluation.

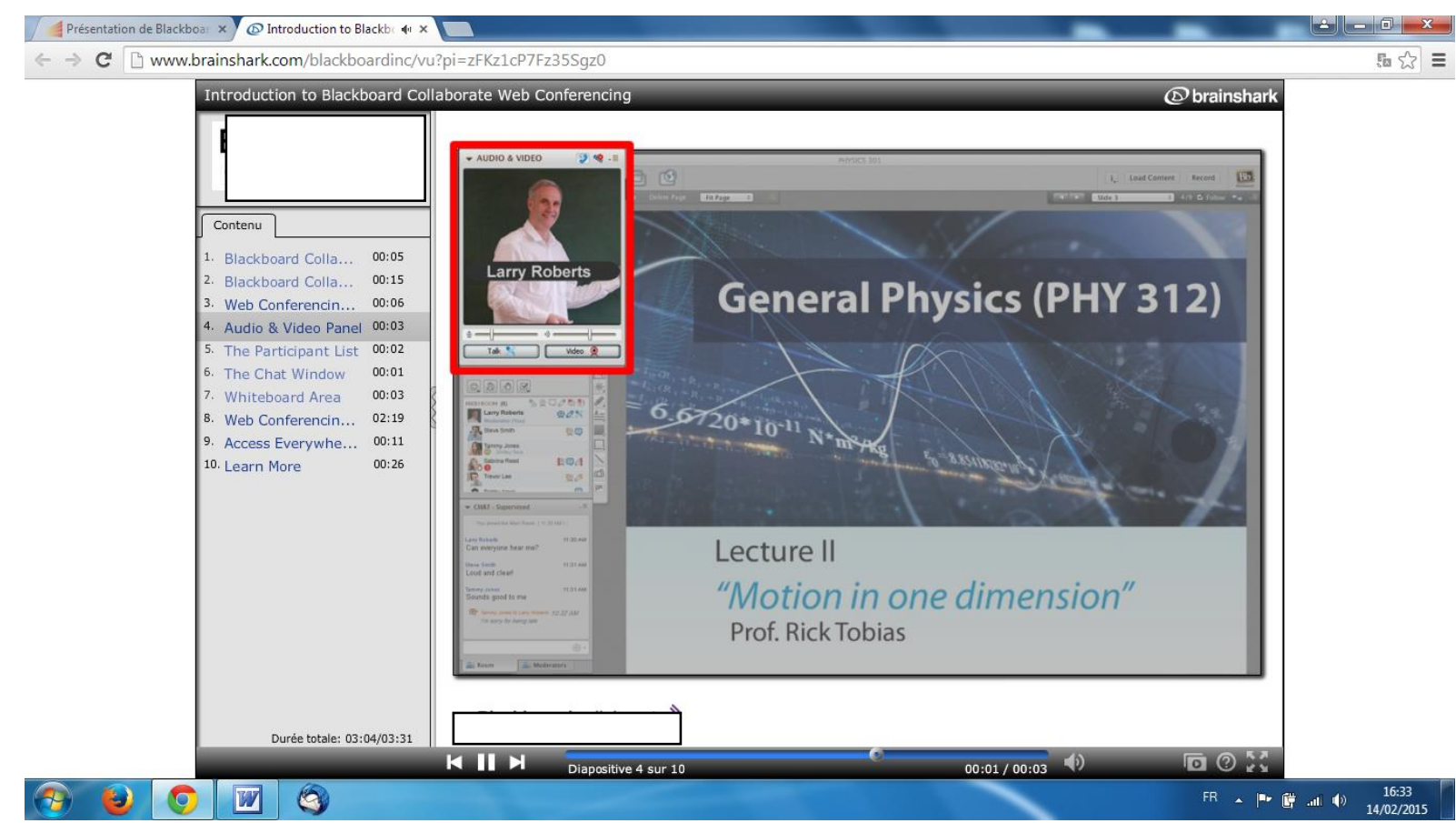

\section{Une classe virtuelle}

Si nous étions le « chef de projet» de cette ingénierie didactique, il nous a fallu valider l'idée en construisant une maquette réelle. Pour cela, nous avons mis par écrit nombre de données comme le marketing et la comptabilité de l'ingénierie de formation prédéterminée par les formulaires internes à l'administration, mais aussi une partie de l'ingénierie pédagogique : le déroulé du mémoire collectif et les modalités de travail à distance. Il nous a été demandé par voie hiérarchique de procéder à un enregistrement d'une simulation de soutenance collective à distance par classe virtuelle et c'est là que nous sommes entrés dans la phase coopérative de l'ingénierie didactique que nous voulons présenter. 
Nous avons en effet sollicité trois collègues pour former un groupe, deux prenant avec nous le rôle d'un apprenant à distance participant au mémoire à défendre oralement, un se posant en évaluateur devant qui soutenir le travail à distance. Nous avons grossièrement écrit les scripts de chacun pour que la préparation de l'enregistrement se fasse au mieux : nous avions en effet à former certains des collègues à l'outil de classe virtuelle, à expliquer à tous le projet de mémoire collectif et l'opération de soutenance collective à simuler. Ceci a fait l'objet d'une réunion préparatoire, puis d'un premier enregistrement de test et enfin un enregistrement définitif (qui fut ensuite retravaillé par le service de la production audiovisuelle pour en améliorer la qualité et le rendre présentable en réunion).

Question de recherche : comment modéliser l'organisation d'un groupe de travail ?

La dimension coopérative de notre ingénierie didactique apparaît donc à plusieurs niveaux : d'abord par l'insertion dans une ligne hiérarchique forçant la coopération entre acteurs dépendant les uns des autres verticalement, puis par la fusion d'un groupe de travail relativement éphémère (deux réunions pour un enregistrement final dans l'espace d'une semaine) et de même niveau hiérarchique indépendamment du projet.

C'est cette deuxième coopération que nous voulons étudier. Comment s'est organisée la coopération dans le groupe de travail ? Comment son fonctionnement l'a structuré et a permis une construction qu'il nous reste à déconstruire pour mieux modéliser ? Notre question de recherche est donc centrée sur la problématique de la modélisation de la coopération. Comment schématiser les processus de construction d'un groupe de travail dans une opération conjointe d'ingénierie didactique ?

Nous utilisons à escient le terme d'opération conjointe, puisque c'est celui qui est classiquement retenu par les théoriciens (dont Chevallard, 1992) de l'ingénierie didactique quand ils étudient les prestations combinant plusieurs enseignants ou un enseignant et un intervenant extérieur. Les particularités de notre cas d'étude sont qu'il s'agit d'une organisation éducative industrielle (s'opposant à l'artisanat de la plupart des pratiques Caron, 2010) avec forte division du travail. Nous étudions l'ingénierie primaire dans la préparation d'un exercice évaluatif où les rôles d'élèves et de correcteur sont distribués parmi des collègues professionnels occupant tous des fonctions à responsabilités dans l'enseignement supérieur à distance.

Cadre théorique des sciences sociales du fait éducatif 
De ces données primaires concernant la démarche d'observation participante en institution d'enseignement et une problématique centrée sur l'organisation d'un groupe de travail, on déduira aisément qu'il s'agit d'un travail de sciences sociales appliqué au fait éducatif. L'ethnographie est ici une «ethnodidactique» (néologisme récent doublant l'ethnopédagogie et l'anthropologie pédagogique) qui s'intéresse à la formation à distance d'adultes. Notre cadre théorique est celui de culture d'action commune, d'analyse des activités, d'identité et de communication.

Notre théorie de l'action (au sens du terme theorein, désignant en grec ancien l'observation) est donc plus qu'une praxéologie simple retour d'expérience mais bien une analyse ayant recours à des concepts scientifiques et s'inscrivant dans un programme de recherche.

Il s'appuie, qui plus est, sur notre formation préalable à la recherche au département de sciences sociales de l'École normale supérieure et aux travaux que nous avons menés dans plusieurs institutions en rayonnant autour de la première (mémoires à l'École des hautes études en sciences sociales, au Collège de France, travaux pré-doctoraux pour l'École nationale des ponts et chaussées et l'Écoles des hautes études commerciales). Nous avons en effet publié trois livres d'ethnographie des organisations (Marty, 2002, 2003, 2005) se dirigeant progressivement vers l'ethnographie des organisations éducatives et enfin l'ethnographie de l'éducation dans les organisations (Marty, 2014, 2015). Les auteurs au fondement de ces ouvrages et que nous réutiliserons ici sont les sociologues comme Moreno (1987) pour le sociogramme, Halbwachs (1994) pour la mémoire collective, Crozier (2014) pour les stratégies individuelles, Hall (1992 et 2014) pour la proxémie, Latour (2005) et Rabardel (1995) pour les actants non-humains ajoutant une dimension anthropotechnique.

Le présent chapitre vise à reprendre et intégrer l'apport de ces différents auteurs dans un modèle unique de la coopération. Nous allons en présenter les différents éléments successivement avant de les synthétiser en un panoptique heuristique dont nous proposerons une discussion épistémologique en conclusion.

\section{La mémoire collective comme convergence venue du passé}

\section{La dynamique de l'analyse organisationnelle : des organes à l'organisme}

L'analyse organisationnelle que nous proposons vise à disséquer le tout coopérant pour saisir la structure liant tous ses éléments. Nous présentons l'organisation comme un 
processus, un mouvement s'inscrivant dans le temps, tel que le signale le suffixe -tion. Nous allons donc partir des différents organes qui s'assemblent et s'organisent dans le temps pour former un seul organisme.

Le modèle dynamique se base sur une division du travail, d'abord verticale entre le chef de projet qui réunit ses collègues et les guide dans l'idée qu'il s'était faite de l'enregistrement de la maquette d'évaluation, puis une division du travail horizontale entre compétences complémentaires : ainsi chacun joue un rôle différent (étudiant ou évaluateur) et tous apportent leur particularité.

La division du travail se fait progressivement par négociation des rôles pour ajuster les différentes personnalités les unes aux autres, chacun développant les traits et savoir-faire appréciés positivement par ses collègues et inhibant ceux qui sont critiqués. Le tout se forme ainsi et l'unité de tous est progressivement trouvée dans la coopération.

\section{La conformation par une culture d'action commune}

La forme prise par l'organisation est ainsi une conformation des formes individuelles, tirant sa richesse des différences idiosyncrasiques. Il existe toutefois une préformation qui uniformise chacun des employés: tous se fondent dans une culture d'action commune (Barbier, 2010) qui, certes admet des difformités et différences, mais a une tendance homogénéisante.

Cette culture d'action commune est celle de l'enseignement. Les collègues mobilisés ont des diplômes (doctorat, grande école) ou des concours (agrégation du secondaire, professorat des écoles) qui les insèrent dans une culture professionnelle avec ses arts et ses métiers. Tous ont été confrontés à des élèves et ont acquis l'art pédagogique par la pratique, ils sont habitués à la relation éducative en présence avant de l'organiser à distance. Ils partagent le métier d'enseignant, certes dans des disciplines différentes (gestion, mathématique, philosophie), mais avec la même méthode scolaire et universitaire comme métier.

De là découlent des valeurs, règles et normes communes qui homogénéisent les réactions des uns et des autres dans l'action commune. Plus qu'un vivre-ensemble lié à une culture nationale au travail (tel que décrit par d'Iribarne (1993), Hofestede (2010)), c'est un agir-ensemble dans une culture professionnelle s'appuyant sur des outils (la salle de classe, la leçon, le document papier ou numérique, etc.) et une expérience commune. La culture 
d'action commune professionnelle dépasse parfois celle liée à la citoyenneté nationale, un peu comme la langue propre à une discipline académique constitue un genre (Bakthine, 1984) qui peut être traduit d'une langue nationale à une autre sans perdre son identité (liée à des mots techniques, des modes de raisonnements, etc.). Ainsi ce ne sont pas uniquement des techniques de corps maussiennes ou des outils mécaniques qui sont partagés, c'est aussi un imaginaire, des affects et une perception commune de la réalité qui constitue la culture d'action commune. Ici l'andragogie, la valeur européenne de l'apprentissage (tout au long de la vie), le sentiment d'appartenance à une industrie éducative diffusant les connaissances dans le souci du bien commun, constituent un champ de valeurs communes qui préforment les collègues avant l'acte coopératif et facilitent la conformation. Le groupe est en quelque sorte préconstruit dans le sens où les éléments à assembler ont tous une forme compatible entre elles.

\section{Un sociogramme en lieu d'organigramme}

Pour formaliser visuellement cette forme commune, nous présentons la structure informelle du sociogramme qui porte la mémoire collective des habitus partagés entre relations amicales. Dans le schéma ci-dessous, chaque liaison pointillée entre des individus devant leur ordinateur exprime une relation positive.

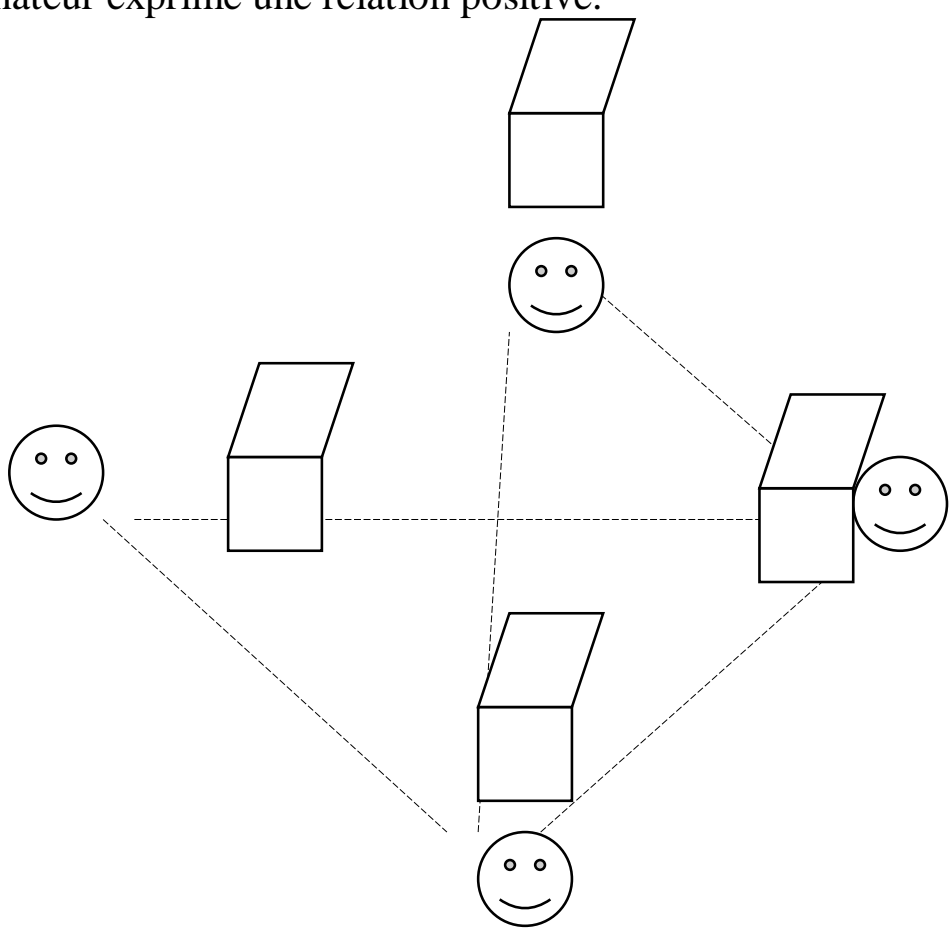


Le coopérant de droite est relié à tous : c'est en effet la collègue la plus ancienne et qui a des affinités avec tous. L'individu du bas serait, dans un organigramme formel (qui n'a pas lieu d'être ici du fait du caractère éphémère de l'organisation), le chef de projet: le sociogramme le réunit donc à tous les autres participants car il a des liens fonctionnels avec chacun. Les deux autres collègues, en haut et à gauche de l'écran, sont reliés à tous sauf entre eux : ils sont récemment arrivés, ne travaillent pas dans des bureaux proches n'ont pas d'affinité particulière entre eux.

La coopération dans une action commune de classe virtuelle a ainsi la valeur principale de boucher le trou structural du réseau dessiné par le sociogramme entre ces deux participants: en coopérant, ils développent des liens et facilitent la transmission de l'information dans le service et donc optimisent son fonctionnement interne en ajoutant de la coopération à la compétition qui avait lieu jusqu'alors.

\section{Une (ré)union par la force des choses}

\section{Le dispositif et ses actants}

Le sociogramme lie uniquement des humains. Une vision plus large des rets est cependant le réseau foucaldien: le dispositif - terme souvent repris dans la langue vernaculaire des professionnels de l'enseignement à distance étudiés - lie des humains et des non humains : les hommes travaillent sur des ordinateurs avec la logique de leur logiciel et en particulier celle de la classe virtuelle. Si la réunion préparatoire place tous les participants dans la même salle physique, elle se déroule aussi dans une salle virtuelle qui réunit leurs avatars. Ils y figurent par le biais de leur webcam, réunis autour d'un seul document partagé avec la possibilité de parler au microphone chacun à leur tour et de tchater en parallèle. Les actants sont donc aussi bien les murs de la salle de réunion que les lignes de codes qui les enserrent.

Nous représentons cette force des choses (salle de réunion, classe virtuelle, logiciels) qui réunit les coopérant par le schéma ci-dessous où les humains sont dans un cadre d'objets présents les entourant et assurant leur cohésion. Nous avons fusionné les murs de la salle de réunion avec ceux de la classe virtuelle en un même rectangle qui contient les humains. Les objets sont pensés comme permanents, force présente à tout instant pour relier les hommes par opération proxémique. 


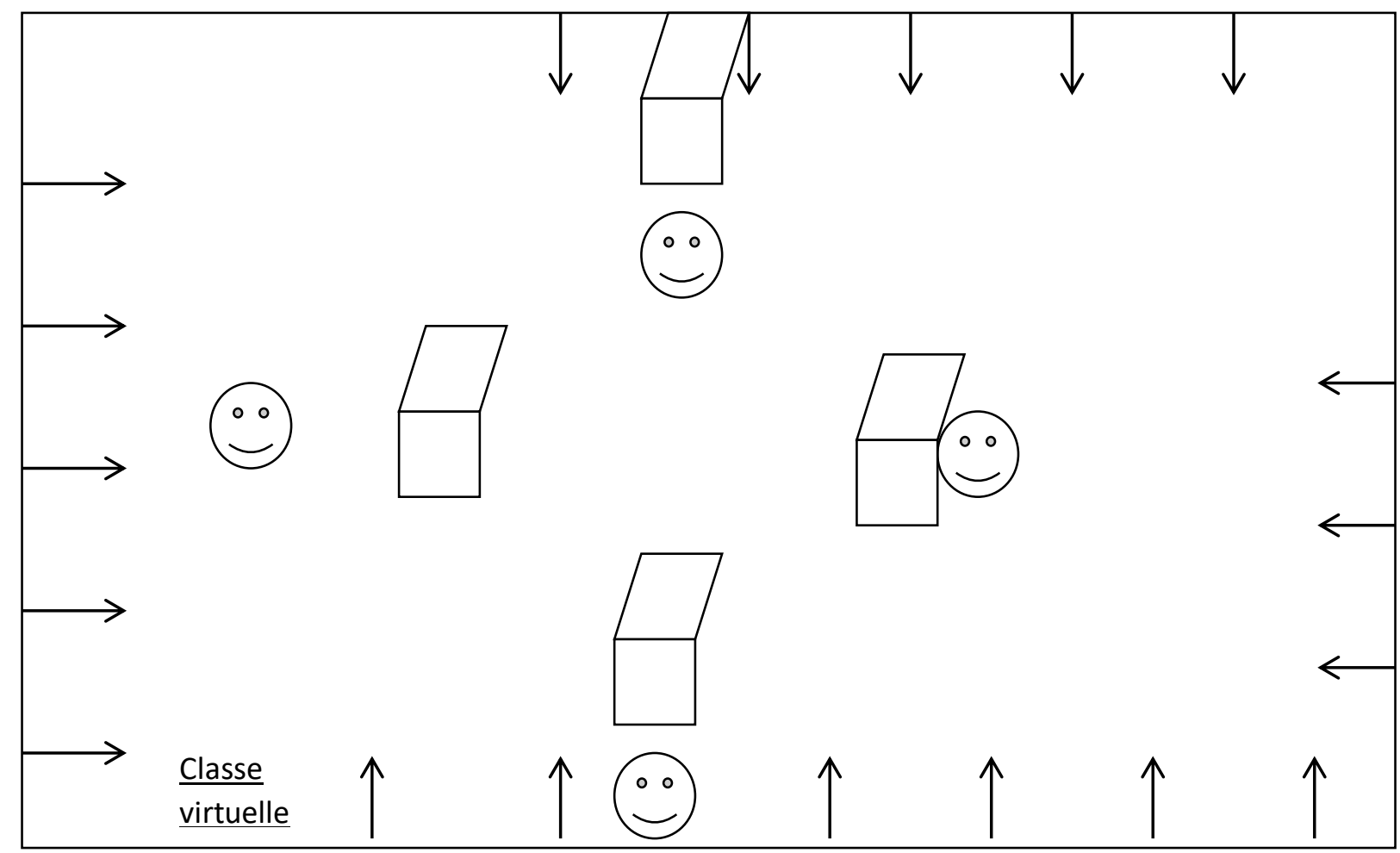

\section{Le cadre présent des actants non humains : la force des choses}

\section{Le psychosocial encastré dans le physique}

Cet aspect sociotechnique, ou anthropotechnique, est suivi, comme nous l'avons montré (Marty, 2002), par un encastrement du psychosocial dans le physique. Ce sont d'abord un espace et un temps qui sont communs aux hommes et que l'anthropologie (Hall, 1992, 2014) place comme premiers. L'endroit de la réunion cadre les interactions qui s'y déroulent. La disposition des tables et des chaises détermine en partie les jeux de regards et les prises de parole selon les possibilités de communication qu'elle ouvre ou ferme : deux personnes face à face communiquent plus facilement que deux personnes côte à côte. Dans la coopération autour d'une ingénierie didactique, on s'aperçoit que le chef de projet est placé à gauche de la collègue la plus âgée, pour éviter une opposition frontale des deux individus ayant le réseau le plus développé dans la situation. La porte de la salle est fermée et isole l'espace physique des interventions extérieures, renforçant l'intimité collective et permettant d'échanger des informations plus librement dans cet endroit isolé et sécurisé. 
Au-delà de l'espace-temps physique qui cadre les interactions sociales, l'espace-temps virtuel du logiciel détermine aussi les facultés psychiques mobilisées. Goody (1979) a montré comme l'écriture était une technologie de l'intellect qui constituait une raison graphique servant d'appui à la pensée. De même, il est aujourd'hui admis que l'informatique constitue une raison computationnelle qui cadre la pensée de ceux qui utilisent un ordinateur. Dans notre cas, les fonctionnalités ouvertes par le logiciel déterminent des modalités de pensée et d'action : les collègues «lèvent la main » pour prendre la parole au microphone qui leur est attribuée par celui qui joue le rôle de l'animateur de la réunion, ils sont ainsi placés en situation de dépendance pour la prise de parole, dépendance qui n'existe pas dans le monde réel. Ils ont aussi sous les yeux un document commun qui fixe l'attention collective sur une même image et un même texte que seul l'un d'entre eux fait défiler. Ils sont donc psychologiquement impliqués par l'audio et le visuel uniquement (ce qui laisse de côté l'osmose olfactive du monde réel ou même le tact et le contact). Dans le monde virtuel de l'espace-temps ouvert par le logiciel, certains gestes et pensées sont donc possibles, d'autres impossibles.

Le physique, réel ou virtuel, cadre ainsi le psychosocial, comme cela se voit sur notre schéma où les hommes sont insérés dans un rectangle, désignant la force des choses. Ici les choses sont pensées au présent, une perspective diachronique est cependant possible: l'artefact de la classe virtuelle agit au présent mais a été pensé dans le passé par des ingénieurs qui ont conçu l'engin informatique. Ils ont eu une intention de logiciel qui est instrumentalisée par nos coopérants voulant l'utiliser pour confectionner une évaluation collective, qui sera elle-même instrumentée par les étudiants qui s'en serviront plus tard. À chaque fois une logique de l'usage (Perriault, 2012) détourne les intentions premières et adapte l'utilisation aux contraintes du moment et des intentions en présence. Les actants non humains laissent une marge de manœuvre aux actants humains qui les adaptent à leurs besoins.

\section{Le média : un intermédiaire non neutre}

Il ressort de ce qui précède que toute analyse des tâches et description psychosociale de la coopération sont cadrées par le physique. Lorsque les acteurs interagissent par le média de la classe virtuelle, leurs gestes, leur collaboration et leurs pensées sont déterminés par les actants non humains. Les médias sont donc des intermédiaires non neutres. L'importance du média se voit dans le champ des sciences de l'éducation française par l'évolution du titre de la revue Distance et Savoirs devenue Distances et Médiations des Savoirs, mais aussi par la 
reconnaissance de l'American Journal of Distance Education qui laisse une large place dans ses articles à l'histoire de l'influence des médias sur l'enseignement à distance. Ainsi c'est la question de l'interface entre les utilisateurs qui est posée pour faciliter la coopération.

Nous avons vu comment le bien commun nouant le groupe est essentiellement cadré par la mémoire collective du passé et par les objets du présent. Ces deux forces sont convergentes. Dans cet espace d'activité, chacun est libre de choisir son avenir, d'établir sa propre stratégie et de fixer ses propres buts, convergents ou divergents. C'est ce croisement des stratégies que nous allons à présent décrire.

\section{Divergences et désunion : croisements des stratégies futures dans l'espace d'activité}

\section{Un espace d'activité laissant ouverte des voies de divergences}

Michel Crozier (2014) et Friedberg (1997), ont bien montré comment les acteurs humains dans un système d'interactions avaient chacun leur stratégie pour augmenter leur pouvoir en s'appuyant sur leur compétence (technique, juridique, organisationnelle, communicationnelle) comme sur les zones d'incertitudes qui masquent leur jeu aux autres. Ils peuvent ainsi s'allier ou s'opposer pour parvenir chacun à leur fin. Dans le cas observé d'ingénierie didactique coopérative, trois objectifs divergents sont poursuivis : le chef de projet veut terminer sa maquette au plus proche de son dessein initial, la collègue plus âgée veut valoriser sa participation auprès de la chef de service et a donc elle aussi intérêt à ce que l'opération conjointe se déroule bien - ce qui est pour l'un une fin est pour l'autre un moyen, ce qui signifie une implication différente. Les deux autres participants sont pris dans un emploi du temps serré et veulent en terminer au plus vite (quitte à ne pas achever l'enregistrement) et trouvent souvent à s'allier pour viser ce moyen commun servant leurs fins individuelles divergentes.

Ceci peut se représenter comme suit dans notre schéma : les flèches sont en gras car elles visent des objectifs dans le futur (là où les pointillés des précédents schémas montraient les liens passés et les flèches d'épaisseur simple la force des choses présentes). 


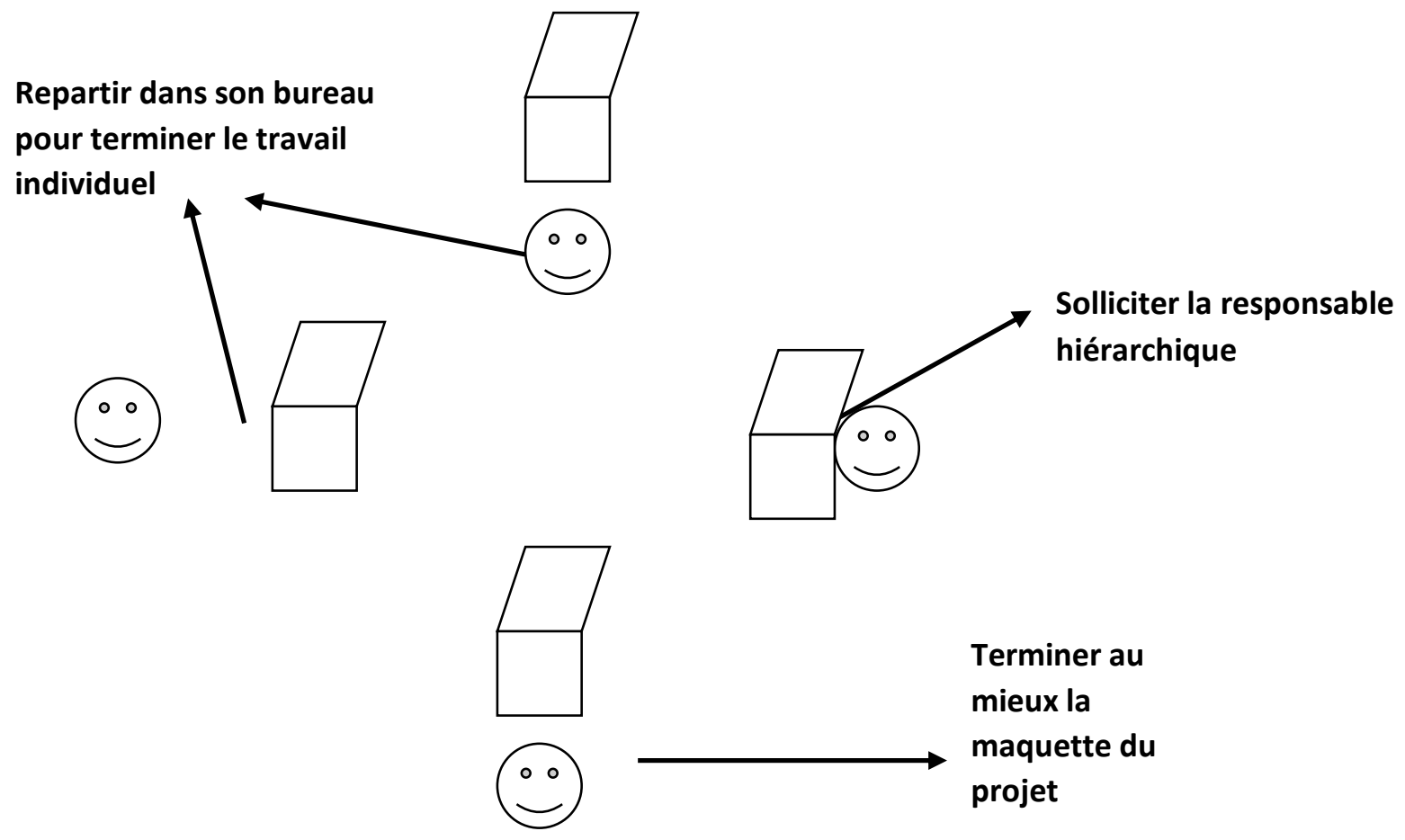

Les stratégies individuelles pour le futur : entre alliances et divergences

Ce qui ne se voit pas : des voix de l'accord aux voies de la discorde

Nous avons montré dans notre schématisation de la coopération plusieurs forces, ici divergentes. Il est cependant des faits qui ne se voient pas mais peuvent pourtant s'entendre. Nous avons en effet dit que l'espace physique comme les relations fonctionnelles dans le projet déterminaient les jeux de regards (plus fréquents entre alliés se faisant face ou entre étudiants qu'avec le correcteur). Ces jeux de regards participent du processus d'organisation par les effets de la distribution des mots : au moment où un mot est prononcé durant la réunion physique, le fait d'associer volontairement ou non son énonciation à un interlocuteur en le regardant contribue à lui assigner un statut. Ainsi, alors qu'un étudiant parle d'un «grand » projet, insistant sur le mot en parlant plus fort et regardant expressément le chef de projet en le prononçant, le chef de projet se voit rehaussé par le qualificatif « grand » du mot «projet », car il se sent visé par la parole. Les jeux d'alliance s'entendent aussi par la même attribution d'un mot à un interlocuteur : les adjectifs possessif («mon», «son») expriment en partie les jeux d'association éphémères qui nouent le groupe. 
Moins visible encore, car purement auditif, les tonalités et le rythme en disent long sur l'organisation et la cohésion du groupe. Le collègue qui joue le rôle de l'évaluateur et qui lance la réunion donne le ton aux étudiants qui parleront après elle. L'étudiant qui présente le projet et fait défiler les pages à l'écran donne le rythme au groupe. Chacun parle alors dans cet espace sonore en improvisant dans la gamme ainsi ouverte. La symphonie générale du début de projet marque un bon fonctionnement de l'organisation où chacun prend la parole à tour de rôle et avec justesse. La fin de l'enregistrement est toutefois marquée par des hésitations, des ruptures et contretemps qui dénotent et permettent d'entendre la cacophonie d'un dysfonctionnement discordant. La désunion s'entend plus qu'elle ne se voit ici. C'est ce que nous avons voulu dire en jouant sur l'homophonie polysémique du son « voi(t/x/e) ». Ici aussi le son qui trahit le sens (ou plutôt la dissonance qui révèle la dissension) est cadré par le physique car la fréquence des interventions doit être audible par la machine, ce qui impose des prises de paroles sensiblement plus lentes que pour une réunion en présence.

\section{Synthèse panoptique : un algèbre du moment ${ }^{54}$}

Mais cessons-là les considérations sur l'entendement commun et revenons à notre visualisation de la coopération. Nous pouvons synthétiser nos schémas dans le panoptique suivant, qui illustre la notion d'algèbre éducatif précitée :

\footnotetext{
${ }^{54}$ Le terme d'algèbre du moment désigne la " réunion » (" algèbre » en arabe comme en mathématiques) d'éléments temporels (nous verrons qu'il peut être synchronique ou diachronique).
} 


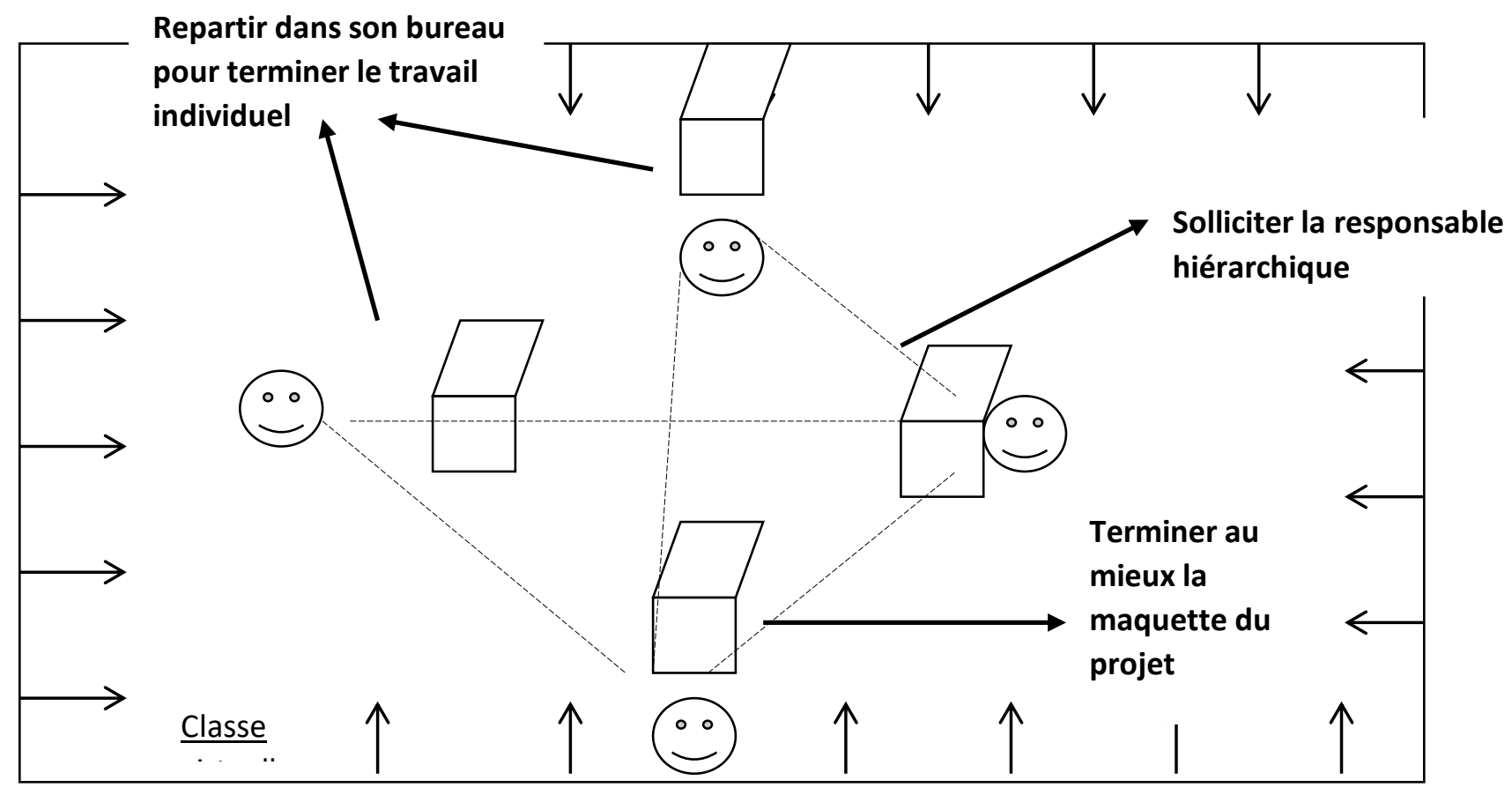

曲 $\frac{8}{8}$

Le passé des individus et du groupe les a conduit à nouer des relations et des habitudes les reliant dans une culture d'action commune

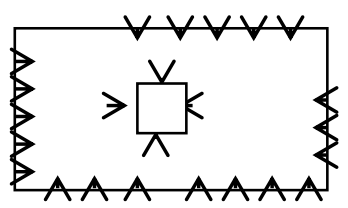

Le présent ou situation commune les réunit dans une pièce autour d'une activité de classe virtuelle tout en leur laissant entrevoir des suites variées

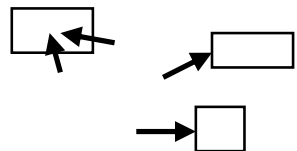

Le futur est marqué par des convergences vers des fins communes pour certains, des divergences vers des finalités variées pour d'autres. 


\section{De la stratification}

La principale valeur heuristique de notre modèle de la coopération est la superposition de trois couches d'analyse (sociogramme / actants non humains / stratégies) dans une dimension temporelle : la mémoire collective et le poids du passé, le présent atemporel des objets, les visées orientant les actions vers le futur. Cette superposition en strates temporelles fusionnées dans un schéma planaire rend le modèle complexe et pourtant réutilisable en de multiples situations de coopération. Nous l'avion ainsi déjà employé dans un carnet de terrain ethnographique d'un groupe d'apprenants dans une grande école collaborant à un projet sur six mois.

Le schéma tel que nous le présentons montre un passé qui soude et un futur qui divise (le mouvement est une scission) ; un autre schéma est possible en superposant des strates temporelles montrant un passé qui divise et un futur qui rassemble (le mouvement serait fusionnel). La psychologie sociale de la coopération en milieu éducatif présentée a ainsi une dimension temporelle - de scission ou de fusion du groupe - qui lui donne la coloration de psychologie sociale du développement d'un groupe. Nous l'avons appliqué aussi bien à une situation d'apprentissage (collaboration d'étudiants en grande école) qu'à une situation d'ingénierie didactique (la coopération ici décrite).

\section{La valeur du présent}

La superposition de plusieurs schémas comme photographies prises à différents instants (avec à chaque fois un passé et un futur dépendant de l'instant présent) peut permettre de comprendre l'évolution d'un individu ou d'un groupe à travers les strates. Ainsi, dans le schéma de coopération tripartite ci-dessous, on place en phase deux (en vert) l'algèbre synchronique, pour le faire précéder d'un moment précédent (en noir) et d'un moment qui lui succède (en bleu). Afin de simplifier, nous ne schématisons que les stratégies et la force présente des choses (nous avons effacé la mémoire du sociogramme pour plus de lisibilité). On s'aperçoit alors qu'en phase une, le groupe n'est pas relié par la salle de réunion ou la classe virtuelle : les coopérants adhèrent juste au projet d'enregistrement que leur a présenté brièvement le chef de projet (individu b). En phase deux, ils sont réunis par une salle de réunion (c'est la force des choses représentée), mais l'accord sur l'enregistrement dans le logiciel est discuté : comme nous l'avons vu ci-dessus, deux collègues (c et d) veulent repartir dans leur bureau pour assurer leurs tâches quotidiennes, la collègue la plus âgée (a sur le schéma) veut valoriser immédiatement sa participation à la réunion auprès de la chef de 
service. En phase trois, finalement, l'accord est trouvé sur l'enregistrement de la maquette comme moyen commun de valoriser l'activité auprès de la supérieure hiérarchique (qui avait commandé l'enregistrement auprès du chef de projet, argument décisif pour l'adhésion collective).

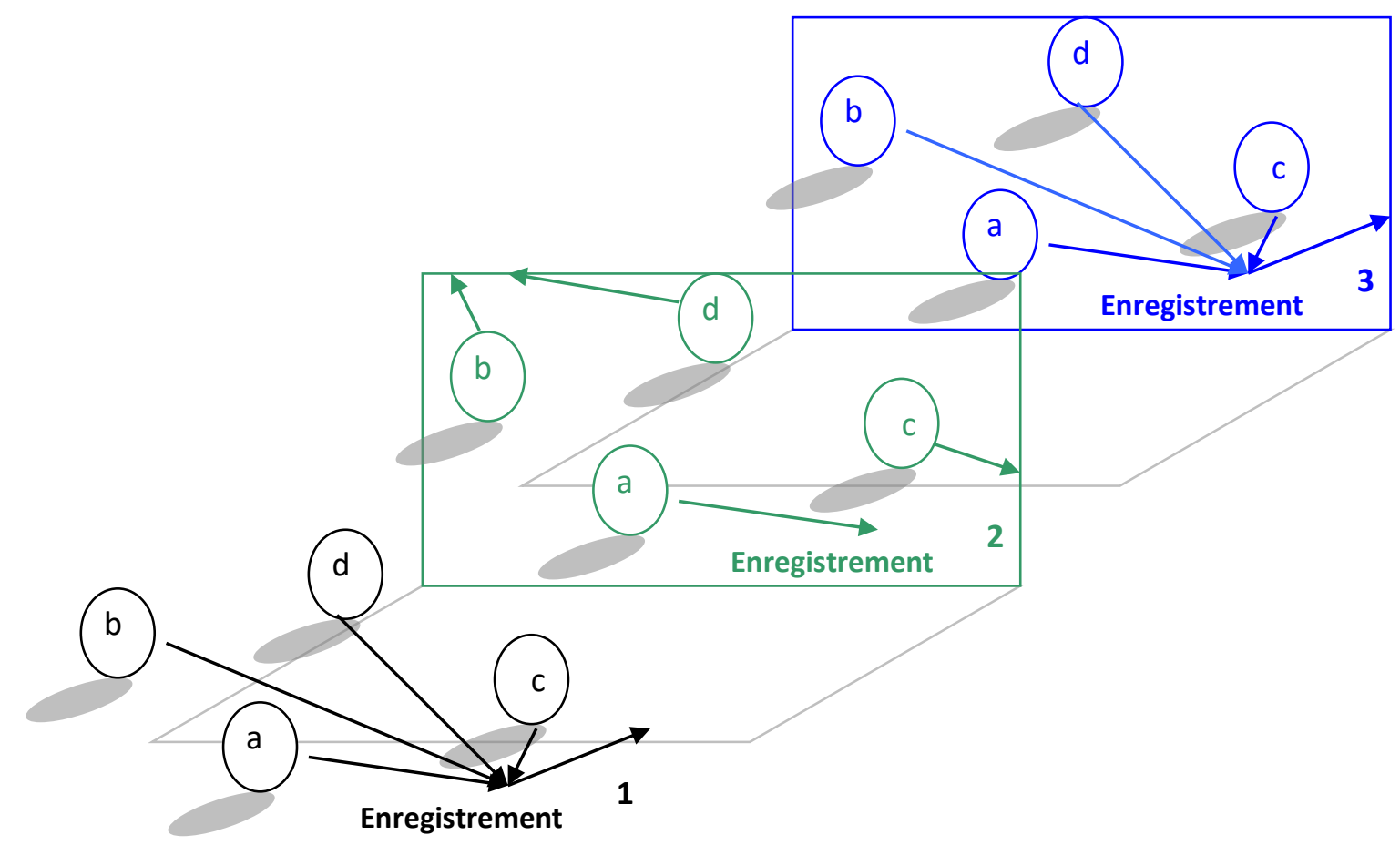

La dynamique coopérative : algèbre diachronique de trois moments successifs

Les groupes d'acteurs du schéma montrent un présent (les choses en présence dans les rectangles) avec ses avenirs (les flèches) qui sont à chaque fois redéfinis pour passer à l'étape suivante. Ceci permet de disséquer les situations et d'éviter les reconstructions simplificatrices par des récits linéaires qui ne présentent qu'un seul passé et un seul futur cohérent avec le mouvement d'ensemble (de réalisation du projet coopératif, de fusion puis scission du groupe par exemple). Ici l'effet est de ramener au présent une dynamique temporelle complexe en figeant les forces convergentes et divergentes qui sont dans un état d'équilibre à chaque instant.

Dans le schéma ci-dessous, nous représentons comment un moment (ici le moment deux en vert) « déteint » sur les autres. Ainsi le chef de projet dans le cercle a dit, au moment deux, que la plus ancienne des collègues a toujours voulu valoriser l'enregistrement auprès de la chef de service (ce qui n'était en réalité pas le cas dans le passé mais exprime son émotion 
négative face au désistement des uns et des autres). Il reconstruit ou révise le passé à cet instant. Parallèlement, au même moment, il perd courage et exprime sa déception que les collègues soudainement effrayés par l'ampleur de la tâche veuillent se retirer et pense qu'il en sera ainsi à l'avenir et qu'il est le seul à adhérer au projet - ce qui s'est révélé faux puisque tous les collègues mobilisés adhèreront finalement à nouveau au projet au temps trois en $\mathrm{y}$ voyant un moyen commun de se valoriser auprès de la responsable de service. Nous avons donc placé en vert des descriptions de stratégies issues du moment deux dans les moments un (en noir) et trois (en bleu).

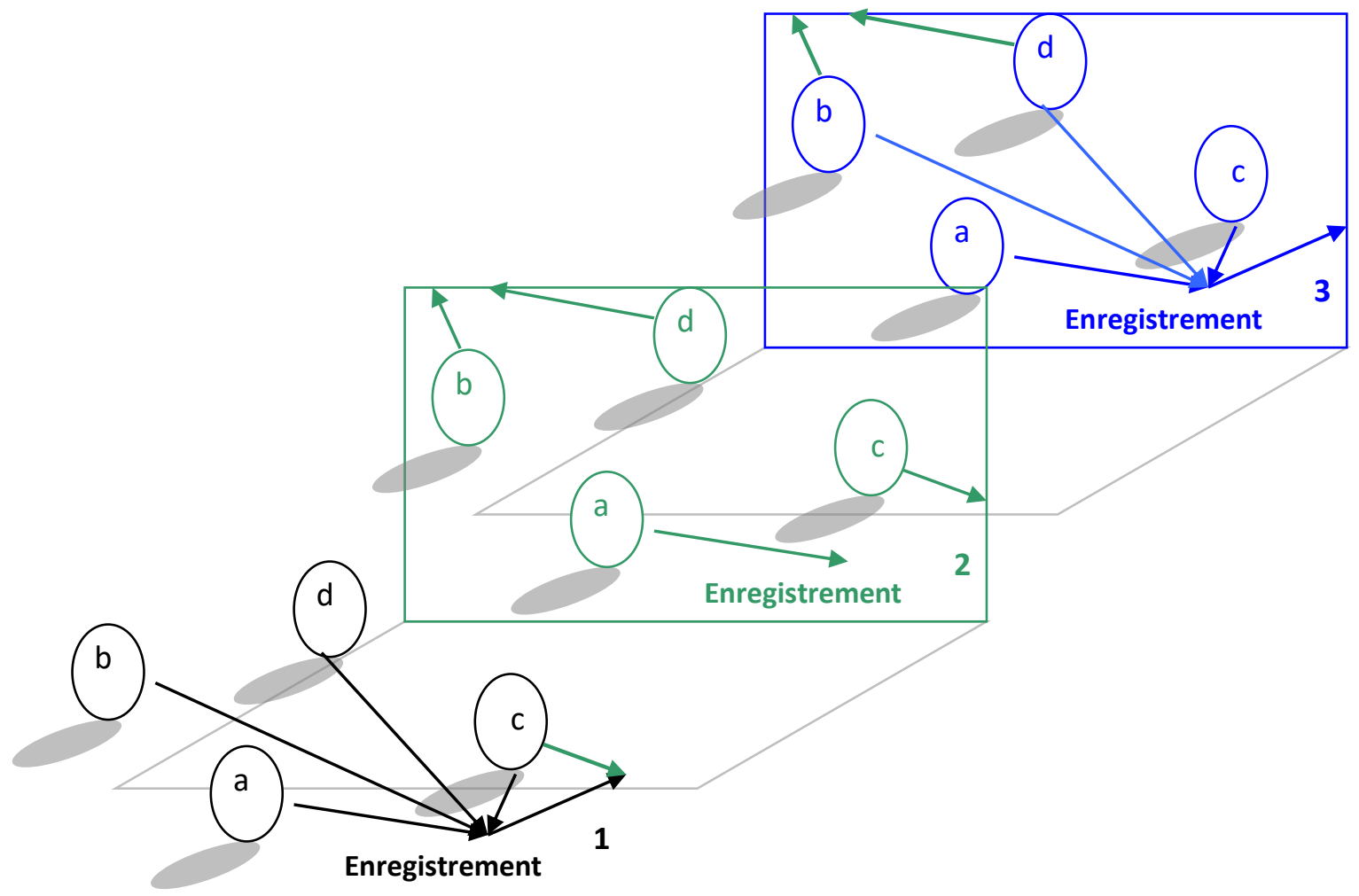

Les simplifications de la coopération : quand le présent déteint sur le passé et le futur

Nous avons-là un exemple simple à schématiser de linéarisation du temps dans un groupe. Les situations réelles peuvent être bien plus complexes du fait du grand nombre de points de vue d'acteurs et des modes narratifs du récit (Ricoeur, 1991) : la déconstruction des reconstructions révisionnistes comme des préfabriqués futuristes est extrêmement difficile à dessiner simplement. Ainsi le chef de projet pourra dire à tort au temps trois avoir toujours été 
en relation avec b (linéarisation des passés dépassés qui oublie qu'un temps cette relation n'existait pas), ou au temps un promettre de toujours garder en mémoire sa relation avec c (linéarisation des passés à venir par promesse de juste reconnaissance historique) ou encore, au temps un, dire vouloir toujours terminer le projet dans les temps imposés par ses collègues c et d (linéarisation des futurs à venir sans présenter les contretemps possibles). Cette multiplicité des cas de figure peut être synthétisée dans un tableau à double entrée.

\begin{tabular}{|l|l|l|}
\hline \multicolumn{1}{|c|}{ Perspective du moment } & Passé & Futur \\
\hline Poment évoqué & $\begin{array}{l}\text { Les passés dépassés : } \\
\text { Exemple de l'oubli présent } \\
\text { d'anciennes relations ou } \\
\text { anciennes stratégies }\end{array}$ & $\begin{array}{l}\text { Les futurs dépassés : } \\
\text { de projets et promesses passées }\end{array}$ \\
\hline Futur & $\begin{array}{l}\text { Les passés à venir : } \\
\text { Refus d'archiver, promesse de } \\
\text { secret }\end{array}$ & $\begin{array}{l}\text { Promesse présente de toujours } \\
\text { vouloir ou d'amitié perpétuelle }\end{array}$ \\
\hline
\end{tabular}

\section{Synthèse de l'algèbre diachronique}

À chaque fois notre méthode est un algèbre du moment dans le sens où elle part du kairos, ou moment présent, pour réunir les forces convergentes et divergentes orientées vers le présent, le passé ou le futur. Nous constatons que le futur, comme le passé, évoluent selon les moments présents, ils sont pluriels et les futurs de l'avenir ne se ramènent pas au futur indiqué présentement, celui que notre grammaire conjugue dans un futur simple. De même les passés ne sont pas simples et réductibles à un temps figé - ce que proposent souvent l'élocution et son écriture mais ne confirme que rarement l'expérience.

La coopération collective paraît rendre incertains les dires des opérateurs individuels les plus fiables. L'emprise d'un acteur sur la temporalité d'une coopération paraît ainsi faible tant l'équilibre des forces est instable et susceptible de revirements et reformulations. 
C'est en se centrant sur le présent des acteurs que l'on comprend la complexité de leurs opérations dans le temps - et de leur coopération encore plus complexe à cause de leurs rapports différenciés au temps. Au lieu de s'arrêter à un discours unique qui place sur une même flèche un passé, un présent et un futur cohérents pour tous, chaque moment et chaque point de vue d'acteur est analysé. Le présent - avec les forces qu'il réunit - apparaît comme une valeur centrale pour comprendre la coopération.

\section{Le positionnement épistémique : ouvertures de l'ethnodidactique}

\section{Auto analyse et distance réflexive : la question de l'étranger}

Nous avons mentionné en introduction notre insertion dans l'ethnodidactique des opérations conjointes. Depuis Homo Academicus, (Bourdieu, 1984) la réflexion sur les pratiques universitaires par auto-analyse est une pratique courante. Le fait, cependant, d'insérer cette réflexion dans les ethnosciences a un double effet de folklorisation de l'enseignement supérieur (ramené à une ethnie savante étudiable malgré ses résistances à l'objectivation et tentatives d'instrumentalisation) - ce qui ne va pas sans poser la question du rendu de l'étude ; mais aussi un effet de tension interne de l'enseignant-chercheur qui sépare ses pratiques de recherche (et la communauté scientifique à qui elles s'adressent) de ses pratiques d'enseignement (et la communauté éducative dans laquelle elle s'insère), ce qui ne va pas sans créer des tiraillements et revirement identitaires : jusqu'où le chercheur peut-il éprouver le sentiment d'étrangeté d'être lui-même un étranger à étudier ?

\section{Vers une anthropologie du didactique}

L'ethnographie des organisations éducatives telle que nous l'avons pratiquée récemment a été une sociologie qualitative de la pratique des sciences de gestion en milieu éducatif. Elle incluait une ethnopsychologie pour comprendre les prénotions, les représentations et les obstacles épistémologiques qui freinaient cette industrialisation gestionnaire de l'éducation comptabilisant la valeur de chaque dispositif. La fusion de la sociologie et de la psychologie fondamentales face aux sciences humaines appliquées en faisait, pensions-nous une anthropologie du didactique.

Il semblerait toutefois que l'anthropologisation de nos efforts dans les ethnosciences doive passer par l'ouverture à d'autres aires culturelles. Ainsi les terrains en France, en partie prisonnier de l'héritage helléno-latin et des concepts de pédagogie, méthode, didactique, 
enseignement, éducation... pourraient aussi s'appuyer sur les analyses venues de la langue savante du monde arabe, de l'autre côté de la mer Méditerranée. Par exemple, la racine trilétère D-R-S condense un vaste champ sémantique autour de la leçon (la darsoun comme genre littéraire), le professeur (le moudarris avec sa geste et son habitus) et l'institution (la madrassa avec son decorum). La comparaison des ethnodidactiques devrait assurer une montée en généralité de nos travaux - passant ainsi de l'analyse à l'algèbre éducatif, avec son idée étymologique et mathématique d'assemblage d'éléments.

Mais dans le cadre de cet ouvrage nous nous centrerons sur le lien entre la compétence (ici coopérative) et les outils (que nous allons voir), institutionnalisé en occident par des organisations telles que le Conservatoire national des arts et métier, qui évoque dans son titre même la complémentarité entre le génie (art) et l'engin (métier). 
État de l'art : littératures, données empiriques et question de recherche

Nous mobilisons un appareillage conceptuel en partie issu d'une même institution d'enseignement à distance française : celui de Jacques Perriault (2012), qui nous a dirigé après avoir fondé un laboratoire aujourd'hui disparu (LABIC) et dont les travaux sur les industries éducatives sont antérieurs aux analyses de sciences sociales du fait éducatif de Pierre Moeglin (2012) accompagnant la revue distance et médiations des savoirs.

Ces auteurs sont en partie héritiers des travaux pionniers de Michael Grahame Moore (2007), fondateur de l'American Journal of Distance Education et éditeur d'un manuel autorisant sa théorie de la distance transactionnelle, ouvrage que nous avons partiellement traduit et publié avec son autorisation (Marty, 2011). Nous reprendrons ici, dans nos définitions, la distinction de Moore entre dialogue et structure pour fonder la différence entre intermédiaires éducatifs et média diffuseurs.

Outre cette littérature liminaire, nous attirons l'attention sur celle qui relève de notre laboratoire d'affiliation, au Conservatoire national des arts et métiers : le Centre de recherche sur la formation (Crf), fondé par Jean-Marie Barbier, directeur de la collection Formations et pratiques professionnelles aux PUF. Au-delà du cadrage scientifique de cet article par ces travaux concernant notamment l'analyse des activités (Barbier, 2011), nous situons ce document dans l'axe de recherche « organisation de la formation » du laboratoire.

Nous décrivons ici les compétences qui font la professionnalité des professionnels étudiés, c'est-à-dire les arts nécessaires aux métiers de la formation à distance. Nous ne pouvons alors que faire allusion au dossier scientifique que nous avons dirigé dans Education Permanente sur «La formation et le théâtre » (Marty, 2013) : reprenant cette même métaphore théâtrale, nous voulons décrire - non pas la «scénarisation » de la formation mais bien les coulisses où les machinistes œuvrent à l'accès au savoir par le public des «apprenants » (« inscrits », «clients » ou « usagers » selon la quadrilogie de la langue vernaculaire). 
Ainsi définie la littérature scientifique éclairant les données empiriques, nous posons une question de recherche pour cet article : comment s'organise l'accès aux savoirs à distance dans un établissement dont le projet est l'enseignement - et dont nous valoriseront les métiers par le simple fait de les décrire ? Nous procéderons en trois temps : après des définitions d'usage, appuyées de schémas explicatifs, autour des notions de « savoirs » et de «médias » (1), nous décrirons en détail l'organisation des arts et métiers de la formation à distance dans un site d'exploitation (2) pour enfin offrir une conclusion synthétique résumant notre apport aux industries de la connaissance.

\section{Définitions : autour de savoirs et médias}

\section{Vocabulaire indigène et scientifique : savoir, enseignement, connaissance, formation}

L'étude des « savoirs » et de leurs médiations nous fait savourer des termes tels que « enseignements » et « connaissances » qui sont largement diffusés dans la langue indigène de l'établissement étudié et pourtant moins utilisés dans les intitulés des revues scientifiques. Ainsi, dans la liste des revues présente sur le site Internet de la section soixante-dix du conseil national des universités français ${ }^{55}$, trois intitulés de revues contiennent le mot «savoir » (Distance et savoir, Savoirs, Les cahiers de la recherche sur l'éducation et les savoirs), mais aucun ne contient des mots plus couramment employés au sein de l'institution étudiée tels que « connaissances ». Ceci se confirme si l'on traduit « connaissance » par «knowledge », lui aussi absent de la liste des revues. Le terme «Teaching» est plus problématique : nous comptons vingt-deux occurrences dans des titres de revue, mais il traduit plus souvent l'acte d'enseigner ou d'apprendre à quelqu'un et non le contenu de l'enseignement ou signe délivré. En langue scientifique anglaise, 'l'enseignant' est plus étudié que 'l'enseignement'. Il semble donc qu'il y ait un décalage entre la langue scientifique et la langue employée dans le quotidien de l'institution. Nous nous tiendrons, dans cet article, au vocabulaire scientifique en employant naturellement le mot savoir et en citant les « enseignements » ou « connaissances » entre guillemets pour faire référence à la langue vernaculaire.

\footnotetext{
${ }^{55}$ Site http://www.cpcnu.fr/web/section-70/rapport-d-activites-et-documents consulté le 23 juin 2014. La liste a été établie en 2011 (elle nomme l'ancien titre Distance et savoirs) et compte plus de 540 titres de revues de sciences de l'éducation constituant un champ de publication scientifique.
} 
Notre étude de vocabulaire doit encore être précisée puisque nous menons notre observation-participante dans une direction intitulée « direction des formations et services » et que notre cadre conceptuel est issu du laboratoire intitulé Centre de recherche sur la formation. Le point commun étant le terme de «formation », à la fois langue de travail sur le terrain et langue d'analyse scientifique. Ce terme de «formation» est présent dans six intitulés de revues de la même liste de la section soixante-dix et sa version anglophone «training » apparaît une fois, dans la revue «Vocational training ». Ce titre de revue nous permet d'avancer une explication sur l'utilisation de ce mot en sciences de l'éducation marquée en France. En effet, depuis la loi de 1971, la formation est mise en avant dans la formation professionnelle continue («vocational training») et reprise aussi bien dans la langue des chercheurs (dont ceux de notre laboratoire) et dans la langue des acteurs de terrain (ceux de notre direction étudiée). Ici, nous prendrons le terme de «formation » dans ses deux sens, soit la « formation » étudiée sur le terrain, soit la formation comme concept scientifique.

Du vocabulaire de Moore à la distinction entre « savoirs médiatisés » et « intermédiaires éducatifs »

Moore propose une théorie de la «distance transactionnelle ». Cet auteur a eu un rôle important outre-Atlantique dans la structuration du champ de recherche portant sur l'enseignement à distance, notamment par la publication d'un manuel dans sa troisième édition. L'article que nous avons traduit de cet auteur (Marty, 2011) propose un modèle visuel synthétique distinguant deux grands axes pour dessiner l'espace de la distance transactionnelle : la structuration des contenus d'enseignements en axe vertical, le dialogue ou communication comme axe horizontal. La différence est nettement marquée entre ce que nous pourrions européaniser sous les idées plus générales de "méthode » (c'est-à-dire de chemin dans la connaissance que les anglais traduisent par l'expression learning path et qui dépend étroitement de la structure du contenu de cours) et de «pédagogie » (évoquant le guidage des jeunes apprenants, nécessitant du dialogue et de la communication entre l'élève et le maître).

Comment allons-nous utiliser ce cadre conceptuel dans le terrain étudié ? Les professionnels de l'enseignement à distance que nous observons découpent officiellement le «produit de formation » qui est l'objet de cette industrie entre, d'une part des « supports de cours » (DVD, livres, documents PDF sur site Internet, etc.), d'autre part des « services 
d'accompagnement» (tutorat téléphonique, classe virtuelle, tutorat par mél, etc.). Nous retrouvons bien la distinction fondamentale de Moore entre la structure du cours (ou méthode) et le dialogue avec l'accompagnant (ou la pédagogie). Nous allons employer cette distinction pour orienter notre angle d'étude des médiations des savoirs dans l'enseignement à distance. En effet, nous observons deux types de média qui correspondent aux deux facettes du «produit de formation » et du modèle de Moore : d'une part les médias qui diffusent unilatéralement des «supports de cours ». Ce sont les médias sur lesquels s'inscrivent la structure du contenu de formation, comme le signe gravé sur un DVD, ou l'enseignement contenu dans un PDF. Le support de cours y est inscrit et structuré définitivement, l' « apprenant » évolue ensuite plus ou moins librement en suivant la méthode proposée. D’autre part les médias qui servent d'intermédiaire à la relation éducative bilatérale, c'est-à-dire qui permettent les «services d'accompagnement». Ainsi le dialogue pédagogique entre le tuteur et l'élève n'est possible que par un dispositif de boîte vocale téléphonique permettant de distribuer les appels, ou encore par l'entremise d'un forum dans l'environnement numérique de travail.

La distinction fondamentale de Moore en deux axes pour étudier l'enseignement à distance (structure et dialogue) peut donc être appliquée à la problématique outillée des médias. Elle se traduit par l'opposition entre ce que nous proposons d'appeler les «savoirs médiatisés » diffusés à distance unilatéralement et les « intermédiaires éducatifs » permettant la correspondance bilatérale, comme résumé dans les schémas ci-dessous :

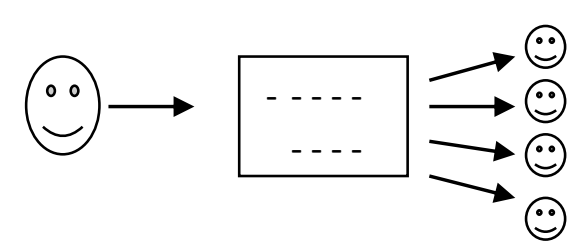

Diffusion unilatérale d'un savoir médiatisé

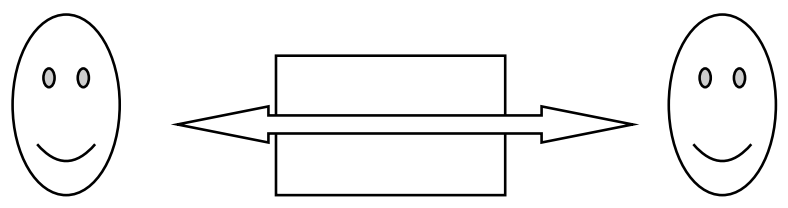

Correspondance bilatérale : les intermédiaires de la relation éducative 
Dans le schéma de gauche, le média (livre, DVD, fascicule de cours) sert de support où s'inscrit le signe de l'enseignement, ce support est rédigé par un faible nombre d'auteurs qu'ils autorisent - et posté à un grand nombre d'apprenants. Les économies d'échelles permettent d'industrialiser ces pratiques et de former de grandes masses d'étudiants. Le cours est conçu et rédigé une seule fois, chaque apprenant aura ensuite sa propre lecture, son propre parcours dans ce cours. La méthode idéale est donnée, les contenus sont plus ou moins structurés et rigides; chacun est ensuite libre d'effectuer son propre parcours librement, en ne reprenant pas ce qui est déjà appris, voire en délaissant telle ou telle partie qui ne fait pas partie de son propre objectif pédagogique.

Dans le schéma de droite, le média ne supporte pas un signe fixé définitivement. La boîte mél, le forum de site Internet, la liaison téléphonique sont autant d'intermédiaires qui permettent une correspondance entre un ou un faible nombre d'apprenants et un enseignant à distance. L'un expose ses difficultés, l'autre lui répond, le premier peut alors répondre par un nouveau problème : l'écho des questions-réponses permet d'individualiser la formation par l'intermédiaire de correspondance. Le dialogue pédagogique permet une relation éducative bilatérale et une individualisation adaptée des parcours de formation.

La distinction entre deux types de médias, fondée sur la théorie de la distance transactionnelle de Moore, rejoint ainsi les recommandations de Louise Sauvé (2014) dans son article pour Distances et médiations des savoirs: enseigner à distance, c'est à la fois produire des savoirs médiatisés à diffuser et à la fois organiser la relation scolaire professeurélève par des intermédiaires éducatifs. Cette recommandation théorique, issue de l'observation attentive des dispositifs d'enseignement à distance, est reprise dans la pratique. En effet, notre terrain empirique institutionnel nous a amené à visionner une vidéo de présentation de la nouvelle organisation («matinale » du 5 juin 2014 où le directeur général présente la nouvelle organisation de l'institution). Cette annonce de réorganisation de l'établissement, dès la sixième minute de la vidéo, fait une référence implicite à l'article précité de Sauvé lors de l'annonce d'une décision pratique de création de nouvelle direction. Cette nouvelle direction ainsi présentée devant prendre en charge les deux dimensions de l'enseignement à distance : cours standards et parcours individualisés, que nous outillons en savoirs médiatisés et intermédiaires éducatifs.

Nous voulons à présent décrire le maniement de ces médias centraux par les acteurs de l'institution. L'enjeu est de montrer les compétences des différents professionnels impliqués (direction mise à part car elle n'est pas l'objet de notre terrain) et, ce faisant, les arts nécessaires à aux métiers de la formation à distance. 


\section{L’art de manier les média : organisation des métiers de la formation à distance}

Notre terrain ne décrit pas le Centre d'éducation à distance dans son ensemble. Nous avons observé les différents arts et métiers complémentaires au niveau d'un des huit sites d'exploitation. Nous avons procédé par observation-participante de longue durée et déclarée selon une épistémologie de l'ethnographie en industrie éducative (Marty, 2014). La récolte des données empiriques ${ }^{56}$ a été faite au fil de l'emploi principal et des problématiques du laboratoire d'appartenance. Nous avons eu l'occasion d'interagir avec quatre sites d'exploitation et exceptionnellement avec la direction stratégique orientant le service public de l'enseignement à distance. Une telle étude complémentaire, au centre décisionnaire de l'organisation, pourrait s'appuyer sur une anthropologie des managers (Flamant, 2002).

Le site que nous étudions est attractif car il est l'ancien siège de l'institution, avant la délocalisation dans le campus du Futuroscope - selon l'histoire officielle telle que narrée dans un document académique sur le portail Internet de l'institution (Bourrel, Vidal, Mahieux, 2008). Le site étudié suit donc les «directions métiers »stratégiques poitevines et les met en œuvre localement. C'est cette activité que nous voulons analyser, en proposant une typologie non-exhaustive de trois arts et métiers correspondant aux trois niveaux occupés de l'immeuble : au rez-de-chaussée le service de la production avec ses «agents de fabrication », à l'étage intermédiaire le service des formations avec ses « responsables de formation chargé d'ingénierie de formation », à l'étage supérieur le service de scolarité avec ses «agents de scolarité ». Ces trois services coopèrent dans le site pour organiser l'enseignement à distance de dix-sept mille étudiants du supérieur-soit l'équivalent d'une université.

Notre problématique est d'analyser les arts nécessaires à la pratique de ces métiers et leur complémentarité organisationnelle. Nous traiterons des arts comme des compétences, savoirs, savoir-faire et savoir-être d'une profession. Les métiers renvoient au triple sens d'une communauté de professionnels, des outils de travail et de l'expérience accumulée par les professionnels maniant ces outils. Nous supposons ainsi que chaque métier a son art qui lui est propre, voire son «bel-art» quand il est bien exercé. Notre sous-question de recherche

\footnotetext{
${ }^{56}$ Notons que le projet d'article est « autorisé » car a fait l'objet d'une demande d'autorisation de divulgation des données empiriques auprès de hiérarchie dans le site étudié. II n'est pas pour autant " instrumentalisé » puisque la même hiérarchie fait confiance au comité de publication de la revue, appartenant à l'institution.
} 
pour cette partie est ainsi : quelles sont les compétences des professionnels maniant les médias d'enseignement à distance ?

Les arts et métiers de la production : compétences professionnelles des diffuseurs de savoirs médiatisés

Nos premières observations de terrain sont issues d'interactions professionnelles avec les «agents de fabrication» en poste dans le «service de production». Les agents se répartissent en métiers traditionnels de l'impression et de la diffusion de document papiers et en métiers plus innovants de production de sites Internet (quoique vieux déjà d'une vingtaine d'année dans l'institution qui est innovante par vocation).

La gestion de documents papier implique de vastes espaces de stockage des fascicules dont l'envoi postal se réparti par pics de hausse d'activité durant l'année (suivant pour beaucoup les rythmes des universités partenaires et des programmes nationaux des concours enseignants) ainsi que de lourdes opérations de manutentions pour imprimer avec les rotatives, assembler les colis, les placer sur les étagères et les poster le moment-venu. L'atmosphère qui y règne évoque celle d'une usine de fabrication industrielle. Les professionnels de ces métiers sont majoritairement masculins et doivent être capables de gérer des chaînes d'opérations manuelles impliquant l'activation de machines à imprimer en masse et le rangement dans des codes abstraits de documents papier dans le respect des délais imposés par l'agenda scolaire.

Dans un autre local du même étage de production, les professionnels qui travaillent à la fabrication des documents (mise en page, relectures techniques, etc.) et surtout ceux qui façonnent les sites Internet (environnements numériques de travail incluant forum, tutorat, documents en ligne, classes virtuelles, etc.) et les matériels audiovisuels (vidéos, tutoriels) oeuvrent dans les arts techniques du numérique et ont pour métier l'informatique (certains collaborent directement avec la gestion des systèmes d'information du site). Les compétences requièrent des clics multiples et complexes et la maîtrise des logiques variées des nombreux logiciels utilisés. L’inscription des savoirs dans les médias pour une diffusion en masse passe ici par l'art de maîtriser l'outil - ou métier - informatique.

La «transformation »stratégique de l'établissement vers un avenir numérique passe par une évolution des arts et métiers de la production de la première catégorie vers la seconde. Ceci implique des évolutions de carrière dans le service lorsque cela est possible et donc l'apprentissage de nouvelles compétences. Cette transformation, qui fait partie de l'évolution 
naturelle de l'institution constatée dans l'histoire officielle durant ses soixante-quinze années d'existence, est accentuée par le plan stratégique en cours. Elle se manifeste par une valeur changeante des arts et métiers sur le terrain et par des opérations de (re)conversion. Notre description statique durant quelques années d'observation (2011-2014) inclut modérément les reconstructions historiques et les projections futuristes de la période - elle ne prétend pas relater intégralement l'histoire dynamique de l'institution (histoire totale du flux de ses passés, de ses présents et de ses futurs, variant selon les moments). Nous nous centrons essentiellement sur l'analyse des activités éducatives et l'organisation des différents services actuellement en présence.

Les arts et métiers de la scolarité: compétences professionnelles pour utiliser les intermédiaires éducatifs

Il n'est pas d'enseignement à distance sans enseignants et enseignés, professeurs et élèves, formateurs et apprenants. Certes les savoirs médiatisés peuvent être diffusés largement auprès des inscrits, mais encore faut-il gérer les inscriptions pour leur adresser le bon support au bon moment. Et l'opération est encore plus délicate lorsque la relation éducative s'intensifie par le jeu des correspondances formatives. C'est là qu'interviennent les « agents de scolarité » dont l'art est de mettre en relation l'apprenant avec la bonne formation - en l'inscrivant dans la base de données de gestion automatisée des élèves - voire avec le avec son correcteur - par l'intermédiaire du logiciel « Copies-en-ligne ».

La problématique est la même que pour la production : les évolutions informatiques, depuis les bases de données et la bureautique des années 1980, jusqu'à la boutique en ligne permettant le commerce électronique actuel, supplantent peu à peu la gestion de dossiers d'inscription sous format papier. La mémoire n'est plus archivée dans les armoires sous forme de formulaires manuscrits, mais est inscrite dans le réseau informatique collectif de l'établissement. La compétence de gestion d'une base de données est donc primordiale pour organiser la scolarisation des apprenants par les intermédiaires éducatifs. Et ce bien que les anciens savoir-faire ne soient pas désuets : la relation téléphonique individualisée permet de résoudre les problèmes de scolarisation d'un cas complexe en échangeant plus d'informations dans un temps bref que par mél ; une lettre reçue par la poste est encore nécessaire pour des inscriptions de dossiers nécessitant la coopération avec d'autres établissements publics comme les universités partenaires. 
Les arts et métiers de la scolarité s'accumulent ainsi par strates avec le temps, les fondations des anciennes compétences graphiques et verbales soutenant les apprentissages professionnels plus récents comme le maniement d'intermédiaires éducatifs informatiques bases de données et logiciels avec lesquelles la jeune génération est plus à l'aise. L'expérience générale de la gestion des inscrits par les anciens prépare l'expertise localisée dans tel ou tel logiciel des nouveaux. Les vieilles armoires de fer se vident mais restent bien présentes, derrière les ordinateurs qui se multiplient. Les lieux de stockage de l'information concernant la relation éducative ont évolué, les plus récents médias permettant un traitement collectif plus rapide et des opérations plus complexes.

Le mode d'existence des objets techniques (Simondon, 2012) que sont les intermédiaires éducatifs passe donc par une individuation discutable : leur utilisation-même est collective et «l'équipe de la scolarité » est marquée par une organisation du travail éducatif basée sur la division complémentaire des logiciels. Chaque logiciel est un métier nécessitant un art particulier pour le manier. La bonne communication entre les interlocuteurs permettant de résoudre les problèmes d'inscription ou de redondance. La sensibilité esthétique à l'unité de l'outil informatique pose alors le paradoxe du réseau: cet objet technique, contemporain des analyses de Simondon bien qu'à l'époque non encore actualisé sous la forme du réseau informatique, peut difficilement être appréhendé dans une unité indivisible. Il est, a minima trois pôles reliés par trois arrêtes dans la théorie des graphes, a maxima une infinité de pôles et d'arrêtes. Mais réduire le réseau à une triade est le rendre inutilisable à l'échelle des arts et métiers informatiques de l'enseignement à distance. Aussi les compétences professionnelles des intermédiaires éducatifs supposent-elles un bon maniement des réseaux informatiques et des bases de données, sans pour autant les réduire à quelques éléments : l'utilisation simple de l'outil ne doit pas réduire la complexité de l'objet-même.

Compétences organisationnelles des cadres intermédiaires : les arts et métiers des « responsables de formation chargés d'ingénierie de formation »

Entre les deux étages de la production (de savoirs médiatisés) et de la scolarisation (par les intermédiaires éducatifs) se trouve un étage de coordination pour chaque formation. À cet étage collaborent les «responsables de formation chargé de l'ingénierie de formation » (nous insistons sur le terme de formation qui est repris dans notre laboratoire : le «Centre de recherche sur la formation »). Ces cadres intermédiaires, qui participe de notre identité à nos 
heures rémunérées, ont de multiples fonctions : «chef de projet », « manager transversal », «pédagogues »... L'intitulé principal évoque la capacité à répondre de formations et de s'ingénier à en créer. L'engin "formation », au cœur des responsabilités et des activités d'ingénierie, est appréhendé sous plusieurs formes : formalismes administratifs selon des données économiques (coûts, revenus, rentabilité du «portefeuille de formations») et formatage pédagogique selon la discipline d'appartenance. Le bon maniement de l'engin suppose des interactions fréquentes et efficaces avec les professionnels de la production et de la scolarité des autres niveaux de l'immeuble.

Le cœur de l'organisation de chaque formation est donc au centre, entre les deux autres étages du site d'exploitation. Les activités quotidiennes sont variées, quoique les missions, telles que décrites dans la fiche de poste officielle commune à tous, évoquent la veille, la création de nouveaux projets et leur réalisation avec des partenaires éventuels, l'assurance de la bonne qualité pédagogique des formations existantes - ce qui implique de piloter les équipes et de communiquer pour promouvoir l'offre. Ces arts multiples comprennent un savoir-être de sociabilité communicante, un savoir éducatif à la fois technique et pédagogique et un savoir-faire relevant de la gestion de projets complexes et collectifs. Le métier se confond avec la formation en elle-même, dans ses dimensions pédagogique (gestion des relations éducatives entre enseignants et enseignés), méthodique (recrutement des auteurs pour édition de supports de cours) et organisationnelle (gestion des projets de formation) ou administrative (car l'activité est cadrée par des logiciels et des personnels de gestion des contrats d'auteur, de transmission de l'information aux autres services, etc.).

Les missions identitaires sont la responsabilité de formations, impliquant le renouvellement de l'offre annuellement. Ainsi la formation de préparation à une agrégation implique de contractualiser chaque année des auteurs pour préparer des cours et devoirs en lien avec le thème du concours. Cette activité d'ingénierie est complétée par la mise en place de travaux collaboratifs sur les sites de formation: ainsi le tutorat du forum implique des discussions collectives autour de sujets en lien avec le programme. La transformation du métier implique de passer de cette ingénierie pédagogique traditionnelle à une ingénierie de formation, selon la distinction de Le Boterf (2011). Le chargé d'ingénierie de formation doit alors concevoir et piloter la mise en œuvre de nouvelles formations : gamme de modules pour le grand public, licence en partenariat avec une université, etc. Passer de l'engin pédagogique à l'engin formation implique ainsi une complexité plus grande dans les partenariats - qui sont institutionnels et non plus individuels - et dans le maniement des sommes comptées - qui font 
l'objet d'une «matrice économique et financière", outil de gestion des projets. La dépendance aux directives stratégiques poitevines est aussi plus forte car l'ampleur des projets implique de suivre la ligne édictée par le centre décisionnaire.

Un débat actuel, durant la période d'observation, est celui de la bipolarité entre la « conception» de formation et «l'exploitation» de formation. La répartition de la quinzaine de «responsables de formation chargés de l'ingénierie de formation » du site dans ces deux catégories est en effet questionnée par les agents eux-mêmes. Pratiquant leurs arts multiples dans le métier de la formation qui leur est confié, ils se demandent quelles seront les évolutions de compétences qui leur seront demandées pour s'adapter à l'organisation changeante. Nous sommes ainsi face à un cas de professionnalisation permanente pour anticiper et s'adapter aux évolutions du poste de travail.

Le métier de «responsable de formation et chargé d'ingénierie de formations » est relativement central dans l'institution puisque, à un niveau hiérarchique intermédiaire, il fait converger nombre de directions stratégiques visant à améliorer l'offre de formation. L'art de l'adaptabilité à des contraintes multiples et changeantes passe ainsi par des compétences de communication - requises par la fiche de poste - qui nécessitent un sens relationnel développé. La valeur centrale est ainsi sociale.

\section{Conclusion}

L'apport de ce document de recherche en sciences de l'éducation est de montrer qu'une industrie éducative moderne fonctionne selon la division du travail décrite par Adam Smith dans le cas institué de la manufacture d'épingle. Les métiers sont divisés et se complètent grâce à des agents qui correspondent dans un cadre administratif. Les différents organes humains de l'organisation locale font chacun profession d'un art, ensemble de compétences, qui leur permet d'exercer au mieux les activités liées à leur métier. C'est l'ensemble uni des travailleurs qui fait leur valeur totale et rend les savoirs à distance accessibles au public, que ce soit par diffusion de médias ou par intermédiation.

L'organisation étudiée autorise le service public de l'enseignement à distance, avec ses arts et métiers propres. L'intérêt scientifique de notre étude peut être conforté par l'écho distant d'une école interne (l’École de l'ingénierie de la formation à distance, Éifad) dont l'activité consistait notamment à valoriser et conserver ces compétences professionnelles.

Mais nous nous centrerons à présent sur les métiers ou outils de l'enseignement. 
La loi du 23 juillet 2013 marque un tournant dans la législation française de l'enseignement supérieur : la volonté politique de prendre le virage numérique se traduit par le droit, voire le devoir s'il l'on suit leur contrat d'établissement avec le ministère, pour les universités de déposer leurs Cours en ligne ouverts et massifs (Clom) sur la plateforme France université numérique (Fun).

Quelle leçon tirer de cette évolution des infrastructures techniques (une plateforme comme média national) et juridiques (un impératif d'y déposer les Cloms) ? Ce chapitre vise analyser les différents dispositifs impliqués par le tournant numérique sur les autoroutes de l'information, dispositifs qui mobilisent la vue et l'ouïe puisqu'il s'agit d'audiovisuel. C'est pour cela que nous avons choisi le terme de théâtre - nous référent explicitement à l'étymologie de «l'endroit où l'on voit». Quelles sont les modalités pédagogiques des théâtres numériques de la formation? (terme introduit au moment de l'algèbre éducatif, aussi discuté dans la communauté scientifique de la revue française de pédagogie. À noter aussi, sur la scène française, les machines à apprendre, terme évocateur qui pourrait y être substitué).

Dans cette actualité de pédagogie nationale, l'objectif de notre propos a un double enjeu théorique: il s'agit de croiser les traditions de recherches théoriques à la fois philosophiques et scientifiques. Au-delà du projet d'intersection entre philosophie et sciences de l'éducation, le texte veut que les deux traditions se complètent par réunion.

\section{Les notions fondamentales de " théâtres numériques » et « d'algèbre pédagogique »}

Notre propos s'inscrit dans la continuité d'une première œuvre d'éducation permanente sur la formation et le théâtre $\left(\right.$ Marty, 2013) ${ }^{57}$. Ce dossier développe la métaphore théâtrale pour décrire, expliquer et comprendre la formation : à la vision traditionnelle de l'estrade scénique où apparaît l'enseignant en actes, pour délivrer un message prescrit dans son rôle ; nous voulons ici ajouter un complément numérique. Les théâtres numériques sont ces mêmes lieu d'observation et d'audition que sont les salles de classes, mais médiatisés par des objets techniques modernes : téléviseur, campus numérique, classe virtuelle, etc. Nous aurons à explorer l'appauvrissement sensoriel, limité à l'audiovisuel et perdant l'osmose et le

\footnotetext{
57 Marty O., (2013) «La formation et le théâtre » dossier dirigé dans Education Permanente, numéro 194. France.
} 
bon contact humain des scènes de classe d'antan, en posant la question de l'esthétique pédagogique, c'est-à-dire de la sensibilité à ces nouveaux usages par la communauté éducative.

La notion d'algèbre est plus riche encore puisque ce mot arabe signifiant principalement «réunion» est au fondement de la théorie des ensembles dans les mathématiques supérieures actuelles. Ce texte veut ainsi renouveler les discours scientifiques autour de «l'analyse » par une « algèbre » dont l'ambition serait de viser à l'unité, l'harmonie et la compréhension d'un tout. Ce vocable algébrique est particulièrement important au moment où Philippe Meirieu, dans son cycle de conférences sur les grands entretiens de l'éducation, rappelle que « faire la classe » est l'essentiel de l'art de l'enseignant. Si le métier classique de l'éducateur est un outil composé d'une salle, de chaises, d'un tableau, de crayons, de papier... son art est de réunir en sa présence et son discours l'ensemble des élèves. Là où chacun a un imaginaire extra-scolaire qui lui est propre (car au croisement des influences familiales, de loisirs, amicales, etc.), l'unité autour des programmes nationaux se fait dans la personne du maître dont la fonction est donc essentiellement algébrique.

Ces formules de théâtres numériques comme outil et d'algèbre pédagogique (ou algèbre éducatif, nous n'établissons pas de distinction) comme compétence prennent un sens complémentaire au moment où ils se rencontrent. C'est la notion moderne de distance qui, paradoxalement, les rapproche : le livre, le courrier papier et la correspondance permettaient déjà de se libérer de la salle, théâtre classique de l'algèbre pédagogique. Mais lorsqu'Internet ou des dispositifs tels que la classe virtuelle réalisent un théâtre numérique, l'algèbre pédagogique évolue : l'essentiel est alors audiovisuel. « Faire classe » se réalise alors par une présence médiatique dans ces dispositifs techniques modernes.

\section{Une pédagogie numérique comme problématique commune}

Nous avons commencé à décrire, avec l'appareil conceptuel de Gilbert Simondon ${ }^{58}$, les modalités techniques de ces objets numériques à l'individuation complexe. Les théâtres numériques de la formation appellent ainsi une esthétique aiguisée par la sensibilité à l'image, au son et aux dynamiques d'animation de classe qu'elles permettent. L'attention se porte sur les signes de présence des participants dans une liste avec leur statut, le partage d'un

\footnotetext{
${ }^{58}$ Simondon G., 2012, Du mode d'existence des objets techniques, Aubier. Philosophie. France.
} 
document ou d'application, les questions-réponses orales ou par clavardage, la passation des micros, l'expression par émoticônes, etc. Faire un tout de tous les participants par algèbre pédagogique est ainsi un art qui se conserve avec ce nouveau métier numérique. Autour de cet outil que nous qualifions de théâtral par son essence visuelle, une communauté de professionnels expérimentés se constitue peu à peu. Nous prenons ici exemple les utilisations industrielles au sein d'une institution publique nationale pour les cohortes de préparationnaires à l'agrégation (Marty, 2013) ${ }^{59}$. Une genèse sociale des usages éducatifs des théâtres numériques est ainsi à écrire pour informer au mieux de ces pratiques naissantes prolongeant les traditions théâtrales de l'éducation - et c'est l'ambition de ce texte que de prolonger notre premier ouvrage sur la formation et le théâtre par une réflexion sur le numérique.

Dans un théâtre de formation classique, l'algèbre pédagogique indique que l'activité des élèves est régulée par les verbes actifs suivant : ils peuvent lever la main pour poser une question, écouter, écrire, répondre à une interrogation collective, participer au spectacle instructif d'un autre élève au tableau, lire un document distribué, faire un travail pratique par sous-groupe, suivre une visite hors établissement... Tout ceci évoque les théâtres participatifs qui sont des pédagogies actives consistant à «faire la classe ». Si la classe est défaite : c'est-àdire en difficulté, alors le pédagogue peut retrouver son algèbre, anciennement son latin, en écrivant au tableau l'essentiel pour que tous aient le temps de noter ; ceci pouvant même être préparé avant le cours si la prudence s'impose dans une classe difficile. Le maître est parfois un étrange étranger qui professe des mots familiers : un ancien expérimenté et raisonnable qui attire regards, concentre les attentions pour faire algèbre pédagogique dans le théâtre autour d'une méthode classique. La magie de l'éducation se produit alors : tous sont conduits aux bonnes inductions de formes pendant l'algèbre, qui seront sources de communes déductions ultérieures, adaptées aux expériences individuelles

Le même algèbre pédagogique se retrouve dans un théâtre numérique. À titre de prolégomènes, nous constatons que, là-aussi, les élèves peuvent lever la main pour poser une question. Et ce, soit librement, soit par ordre de parole donnée par l'animateur qui distribue le micro, selon les logiques indigènes retranscrites à l'écran des logiciels. Les élèves doivent aussi regarder le tableau commun (le «partage d'application » qui concentre l'attention de

\footnotetext{
${ }^{59}$ Marty O., 2013, "Industrializing virtual classrooms in distance education ", $6^{\text {th }}$ International Conference of Education Research and Innovation, Séville. Spain.
} 
tous sur un document graphique ou iconique, éventuellement dynamique pour une visite virtuelle guidée sur Internet), ils écoutent, peuvent prendre note sur leur ordinateur, répondre à une interrogation...

Bien qu'il s'agisse d'un «théâtre », l'aspect figuratif visuel est minime : une «webcam » permet de voir le visage du locuteur, animateur ou questionnant. Les expressions faciales confortent alors le ton de la voix et donnent sens aux paroles (sourire, effort crispé sur le visage, perplexité, etc.). Les indications essentielles pour faire classe socialement sont données graphiquement par le statut des participants qui apparaît à l'écran : identification donnée (rôle «d'animateur», «d'organisateur», ou simple «participant » libre d'inscrire son prénom, nom, institution, lieu, etc.), sur lesquels des messages automatiques peuvent être activités à sa demande : coche verte ou rouge pour acquiescer ou nier en cas de sondage collectif (le professeur peut voir la réaction de la classe en son ensemble en balayant ces réponses individuelles du regard), applaudissements / sourires pour l'ambiance générale, demande d'accélération ou de décélération pour participer à la définition collective du rythme d'apprentissage, etc. Ces fonctions sont souvent sous-utilisées et dépendent de la maturité des apprenants à distance - l'art d'apprendre en classe.

L'art didactique moderne permet d'organiser des classes complexes avec ce métier numérique : ainsi la distribution de rôles pour une soutenance collective à distance, par coopération organisée autour d'une division verticale des présentations défendues devant le professeur évaluateur. Un élève mène la classe virtuelle et les autres suiveurs ont chacun leur partie à présenter, ce qui génère une dynamique de négociation de l'implication dans la présentation collective entre les suiveurs par jeu de questions-réponses devant le professeur attentif à l'évaluation collective à distance - la distance étant la principale valeur de l'opération. Un tel exercice d'examen par classe virtuelle évoque les classes complexes préparant aux concours de grandes écoles telles que racontées par le professeur expérimenté André de Peretti. Ainsi, à la minute quarante-deux de la vidéo le vénérable locuteur affirme : «l'organisation c'est l'interactivité ». Cette interactivité se fait entre les élèves et avec l'organe professoral, instrument vocalique sensé, raisonnable et logique. Un théâtre numérique de la formation par classe virtuelle peut donc reproduire les organisations complexes des classes préparatoires aux grandes écoles - comme les pratiques estudiantines dans celles-ci (Marty, 2014) ${ }^{60}$ ou des travaux dirigés d'université.

\footnotetext{
${ }^{60}$ Marty 0.,2014, « Studying (in) a French grande école », Higher education and mobilities, Grenoble (France).
} 
Une place de choix est à accorder au clavardage : contrairement au bavardage qui peut nuire à l'algèbre pédagogique, la numérisation permet un contrôle et une utilisation de la communication entre apprenants. Les élèves peuvent, sans gêner le cours professoral, échanger par clavardage dans la boîte de dialogue dédiée : ils construisent un sens commun dont l'outil garde la trace et qui peut être discuté par le locuteur légitime. Ainsi un contre-sens peut être repris et rectifié. Le sous-discours collectif de clavardage, concomitant au discours légitime du professeur et à ses diapositives, peut être confronté à celui-ci : les chuchotements sont enregistrés et vérifiés collectivement selon les dires du maître. Ceci permet l'entraide pour mieux comprendre le discours principal, voire faire émerger des questions pour le professeur.

\section{Un objectif de bien général conservé : définitions complémentaires autour de l'éducation}

Cet exemple d'une pédagogie numérique par classe virtuelle, illustrant aujourd'hui ce que peut conserver un théâtre numérique quant aux pratiques algébriques bien connues des anciennes générations, pose la question du rapport aux programmes nationaux et internationaux. Les nouveaux outils, aussi mentionnés comme technologies de l'information et de la communication pour l'éducation, servent aussi à sculpter le socle commun composé des compétences primaires et des enseignements secondaire élaborés par l'actuel conseil supérieur des programmes, tout comme les disciplines supérieures qui mènent jusqu'à être le disciple d'un directeur de thèse puis d'habilitation ayant une œuvre à transmettre. Les différents personnels didactiques : professeur des écoles, enseignant du secondaire, maître de conférences ou professeur des universités, ont un degré de liberté plus ou moins grand avec la réalisation de ce programme lié aux méthodes passées (les chemins de connaissance tout tracés) pour les adapter dans une relation éducative (la pédagogie d'une prise par la main d'un groupe voire d'un élève unique pour les plus fortunés ayant un précepteur).

Que le socle pour cette génération française dans l'espace européen soit atteint par classe classique ou par théâtre numérique, force est de constater que le niveau monte et qu'il est dans une dynamique d'ascension : là où seulement plus de quatre-vingt pour cent de la classe d'âge devaient être diplômés du baccalauréat après les années 1980, l'objectif ministériel est, en 2014, que bientôt cinquante pour cent d'une classe d'âge ait une licence universitaire. Avant-même la statue individuelle à ériger sur ce socle, une question étatique 
pourrait être celle du statut de ceux que l'on porte jusque-là : soit la question du vocable et des appellations.

La première appellation classique serait celle d'élève de théâtre numérique : plus qu'un spectateur ou qu'un auditeur, le «participant» (vocable indigène inscrit dans le logiciel) est élevé vers le socle des apprentissages. Certes la distance médiatique permet d'élever aussi les absents, mais ceux-ci ne le sont pas totalement puisqu'ils sont réunis algébriquement par l'audiovisuel exposé. Une fois que les élèves sont élevés sur le socle commun, ils accèdent au statut d'étudiant, apprenant par loisir. Ceci implique d'être dégagé des contraintes matérielles et ainsi le statut d'étudiant est souvent plus haut que celui d'élève. L'étudiant a appris des savoirs : il a appris à savourer une alimentation frugale pour se libérer de cette nécessité biologique et aller vers des prises plus nobles, relevant non pas d'un palais gastrique mais de connaissances par phonétique et iconique. Il s'expose à des sons sensés et des visions («védas » en sanskrit) qui fabriquent un imaginaire commun et font une réalité unique et partagée.

La hiérarchie antique des sens est retrouvée. Au plus haut des visions se trouve la notion platonique d'idée, de forme abstraite. Après avoir appris avec appréhension pour éviter le mépris de la compréhension trop commune, l'apprenant permanent se saisit de réalités purement formelles. Il délaisse le maniement maniéré d'outils faciles - les techniques artificielles pour agir avec les objets mais aussi le vivre-ensemble de la sociabilité avec sa classe d'âge. L'apprenant instruit se concentre sur une élévation intérieure par des paroles et des visions, une esthétique binomiale qui limite la sensibilité traditionnelle à cinq sens, pour devenir une forme commune et adaptable, objet de formation en processus continu. Cette paideia atemporelle est une tension archaïque électrisant les classes virtuelles contemporaines, elle est permise par la distance : un espace-temps privilégié qui autorise l'asynchrone (le livre est démultiplié lors qu'il est électronique) comme le synchrone par interactivité de haute fréquence en endroit séparés (car le numérique est plus nerveux et rapide que la correspondance postale traditionnelle). Notre propos collectif n'est cependant pas de définir les valeurs éducatives d'une paideia numérique mais d'explorer les voies ouvertes par la technique moderne - notamment l'évaluation.

L'évaluation repose en partie sur la notion de média qui est centrale : les intermédiaires éducatifs entre l'élève(s) et le maître qui amplifient, déforment, enregistrent l'activité didactique et son symétrique «paideutique». Le medium central permet la circulation des savoirs, des connaissances ou plus simplement des mathèmes, c'est-à-dire des 
unités d'apprentissage dans toutes leurs formes idéales. La formation par suivi didactique numérique apparaît alors être le processus d'acquisition de ces mathèmes ${ }^{61}$, guidé par le pédagogue sur les chemins de la méthode.

Les signes de l'enseignement à distance, auditifs et visuels, permettent la conduite éducative vers la doctrine, par des personnels qui peuvent avoir l'expérience nécessaire pour exercer cet art ancestral, avec les métiers que leur organisation actuelle leurs fournissent à titre d'outil. Ils deviennent alors organe d'un tout au service de la société civile bien administrée : instrument humain fonctionnant dans une école, un collège, un lycée, une institution d'enseignement supérieur et de recherche, une université, etc. Ils professent ainsi les mathèmes livrés par les générations précédentes ou acquises lors de leur propre expérience vécue, devant le public qui leur est confié et avec les outils de l'organisation - plus ou moins innovante.

La problématique organisationnelle apparaît ainsi dans une dynamique, comme le rappelle le suffixe-même qui achève le mot «organisation »: les organes fonctionnant sont engagés dans des luttes de pouvoir pour optimiser leur activité et les institutions sont en perpétuel mouvement interne, d'effervescence de recomposition permanente - plus ou moins rigidifiée par le droit qui fixe les règles du jeu social.

Une utilisation pédagogique du terme de «sacerdoce » laïcisé (c'est-à-dire rendu au peuple) retient notre attention : l'éducation-séparée peut intervenir en cas de mise à l'écart temporaire pour une meilleure réintégration à des fonctions liées à l'ancienneté acquise durant le parcours hors-classe. Ainsi tel pédagogue polymathe pourra s'écarter temporairement des normes et standards de l'enseignement pour mieux les retrouver ultérieurement, après sa période de probation, si telle est sa vocation première. Dans les anciennes facultés de théologie, la notion de sacerdoce permettait d'accéder à des fonctions telles que la prêtrise, ou de personnel âgé par le poids des années naturelles ou celui de l'expérience artificielle - ce qui peut être un modèle pour les institutions laïques. L'organisation des théâtres numériques de la formation pourrait ainsi remettre en cause la pyramide générationnelle en laissant place à de jeunes pédagogues ayant accumulé une expérience originale, parfois à l'écart des autres, et donc de l'ancienneté utile dans le maniement des outils techniques.

\footnotetext{
${ }^{61}$ Le terme de mathème est un hellénisme culturellement préférable, dans les traditions européennes et occidentales, à la notion indoue de véda - pourtant bien connue du fait du passé colonial en Orient.
} 
Ces considérations linguistiques faisant des allers et retours entre sémantique et sémiologie de l'enseignement ont été l'occasion de présenter des dispositifs techniques nouveaux tels que la classe virtuelle, des théâtres numériques propre à cette ingénierie.

Montrons à présent un projet d'ingénierie qui resitue l'enseignement à distance dans son contexte international et historique. La livraison de ce «produit d'ingénierie », du moins en tant que projet présenté par audition au Ministère de l'enseignement supérieur (nous ne fournirons le complément d'ingénierie par les agents du ministère), permet de donner une vision des activités de conception dans l'enseignement à distance. Après la description de la production en actes, la lecture d'un produit conçu (qui n'est toutefois pas à confondre avec la consommation d'un dispositif mis en place) permet de compléter notre travail ethnographique de présentation de l'ingéniosité en formation. 


\section{Un exemple de conception en organisation éducative : la licence ouverte}

Ce pojet a été reçu au ministère de l'enseignement supérieur en 2016 avec l'appui d'un courier de présentation et en qualité de directeur des formations de l'université Paris 8 Vincennes à Saint Denis. Il est donc un prolongement ultérieur au terrain dans l'enseignement à distance, français.

\section{Faits marquants : les enseignements numériques}

Le développement de l'enseignement à distance passe par le numérique, ansi la création de France Université Numérique permet de proposer au public francophone trois cent cours en lignes ouverts et massifs (Clom) produits en France en 2016.

Ces modules de formations donnent la plupart lieu à une certification attestant de la bonne compréhension du module. Au-delà de simples ressources éducatives en ligne, les cours en lignes ouverts et massifs sont donc des unités d'enseignements avec leurs propres modalités de contrôle des connaissances.

Quelques établissements d'enseignement supérieur (école d'ingénieurs tel Telecom Bretagne) commencent à reconnaître ces modules pour les valoriser dans leurs cursus de formation en leur conférant des ECTS.

Ceci s'inscrit dans une dynamique internationale puisque les plateformes anglophones (Coursera, Edx, Future Learn...) proposent le même type de service souvent grauits à défaut de modèle économique arrêté. Il est donc possible d'être reconnu formé à des enseignements très précis et ce en langue étrangère (anglais, espagnol, portugais, etc.).

Les compétences informatiques et internet sont mobilisées dans ce type de formation qui font appel à la transmission de savoirs stabilisés via des vidéo avec QCM pour vérification de la compréhension mais aussi la construction de savoirs par forum ou exercices corrigés par les pairs.

\section{Proposition : la licence ouverte}

Les Clot (Cours en ligne ouverts à tous, aussi dits Clom, Cours en ligne ouverts et massifs) indiquent le nombre d'heures de travail demandé à chaque étudiant. Par exemple, un nombre standard est 6 heures hebdomadaires pendant 5 semaines, ce qui représente 1 ECTS dans la comptabilité européenne. 
Cette comptabilité pourrait être généralisée pour traduire tous les Cloms en ECTS et financer directement les équipes d'enseignants-chercheurs porteurs d'un projet sous ce format (ce qui serait une dépense d'investissement public pour le service de l'enseignement supérieur).

Ceci permettrait à des étudiants de se former durant plusieurs années et d'accumuler suffisamment de crédits pour élaborer un dossier d'obtention d'une licence standard dite « licence ouverte».

Étant donné la disparité des enseignements disponibles en Cloms et leur niveau général qui est souvent de l'initiation, nous pensons qu'ils sont plus facilement regroupables pour former une licence et non un master qui suppose un niveau allant au-delà par une plus grande spécialisation. Ce qui n'interdit par l'utilisation de Cloms dans des cursus de master ou de doctorat (reconnus par des ECTS).

\section{Ingénierie de la licence ouverte}

Le dossier à soumettre à une université pour obtenir une licence ouverte contiendrait le justificatif du baccalauréat ou son équivalent, l'attestation de réussite aux Cloms justifiant du nombre d'ECTS minimal, dont un minimum en langue étrangère, et d'un projet personnel et professionnel sous forme de mémoire.

Ainsi un candidat pourrait avoir accumulé 150 ECTS dans un laps de temps maximum de cinq années (qui correspondrait à une durée de validité des crédits), dont 30 ECTS obtenus dans des cours en langue étrangère, il s'adresserait alors à un bureau d'orientation centralisé (affilié au Ced ou à France Université Numérique) qui le mettrait en contact avec les universités porteuses de licences ouvertes (dans des structures d'accueil liées à celles de la VAE).

Il s'engagerait alors, avec l'université délivrant le diplôme, à un processus de validation pour obtenir le grade de licence et qui passerait par un mémoire (30 ECTS) dans lequel il préciserait les raisons des choix de ses Cloms, la perspective d'ensemble donnant sens à sa formation et le lien avec ses activités professionnelles passées ou projetées.

Un tel mémoire l'aiderait à donner un sens global à sa licence et à l'insérer dans le cadre national des formations et des types de diplômes proposés en France. L'enseignant en charge du suivi de son mémoire pourrait préconiser jusqu'à 10 ECTS de Cloms 
complémentaires pour obtenir le profil de la licence choisie et faciliter une éventuelle intégration en master.

L'étudiant ne serait inscrit dans l'université délivrant le diplôme que l'année de la soutenance du mémoire.

\section{L'apprenant au centre du dispositif}

L'apprenant suivrait en moyenne et en continu 3 (voire 5) cous en parallèle. Chacun débutant selon son propre calendrier.

Une licence professionnelle ouverte pourrait être composée d'une activité professionnelle et 3 cours en parallèle ( 15 heures d'activité face à l'écran). Une licence générale ouverte pourrait être plus intense avec 5 cours en parallèle.

La reconnaissance des crédits des Cloms lors du projet final pourrait être panachée avec une VAE.

L'apprenant gagnerait à ce dispositif en utilisant les TICE pour se former mais aussi en réduisant ses coûts de transport et d'hébergement vers l'université, en ayant la possibilité de s'organiser pour débuter sa vie professionnelle à côté, ou en menant des vies hors du commun comme le montre le marketing de l'enseingment à distance du Ced via le site Internet Smooz (récits de formation de sportifs de haut niveau, artistes ou voyageurs qui mènent leur passion tout en étudiant à distance).

\section{Un modèle économique pour les Clots}

Une telle proposition offre un modèle économique public pour les Cloms : en favorisant la formation tout au long de la vie, à distance et compatible avec une actviité professionnelle, modularisée et individualisée, le service public de l'enseignement supérieur est très fortement accru. La bascule dans l'université du $21^{\text {ème }}$ siècle et ses nouvelles formes d'apprentissage (mobile, professionnel, numérique, modularisé, international...) est marquée, tout en conservant un système de classement par grades universitaires dont la collation est monopole d'État.

Un financement public des enseignants s'engageant dans la production de Cloms (heures comptabilisées dans leur service d'enseignant, voire primes supplémentaires pour 
l'équipe) serait donc justifié pour ce service public d'intérêt général innovant de grande valeur pour l'insertion dans Europe de la connaissance.

Ce financement permettrait, en plus d'impulser une dynamique de création de Cloms, de l'orienter fortement vers tel ou tel domaine jugé d'intérêt national et en rapport avec la typologie actuelle des diplômes de licence et des compétences.

Un tel projet, eu égard à sa dimension, nécessiterait une mise en place de longue durée (production des textes cadres, production effective des Cloms et mise en place des circuits institutionnels) pour une exploitation valable pour plusieurs décennies. Il placerait la France en avance dans la concurrence mondiale de l'économie de la connaissance.

\section{Données financières}

Le coût estimé de réalisation d'un seul Clom par les équipes d'institutions d'enseignement supérieur est actuellement de 30000 euros (chiffres Université Paris 8 et Cnam).

La mise en place d'une licence dans chacun des quatre grands domaines de l'enseignement supérieur d'ici cinq ans demanderait 1000 Cloms délivrant des crédits ECTS à produire (nous en comptons actuellement 300 disponibles en langue française mais ne délivrant pas nécessairement des crédits). Ainsi, dans chaque grand domaine, l'étudiant aurait le choix entre 250 Cloms.

Ainsi on chiffre à 6 millions d'euros annuels de production de Cloms (1 000*30 000 / 5), qui se traduirait par un surcroît d'activité de France Université Numérique et de bourses à projet de Cloms distribuées aux équipes universitaires.

À long terme, ce sont des économies d'enseignants (puisque les cours sont massivement écoutés par les étudiants, les enseignants peuvent travailler en équipe autour de chacun des projets de cours numériques, et effectuer de la recherche scientifique, culturelle et en lien avec les professionnels), et des économies de locaux (les locaux universitaires sont allégés en partie de l'afflux des étudiants de premières années).

Le coût par étudiant varie selon le nombre d'étudiants et l'on peut anticiper une courbe de pente descendante allant de 30000 euros par an dans les premières années de mise en place 
à 500 euros par an (ce qui est largement en-deçà du coût complet moyen dans les premières années d'étude du supérieur).

Objectifs sur 20 années :

-2020 : 100 diplômés (soit 25 par an)

-2020-2025 : 1000 diplômés (soit 200 an par an)

-2025-2030 : 10000 diplômés (soit 2000 par an)

-au-delà de 2030 : 1000 diplômés par an (une licence coûterait alors 600 euros par an, soit $10 \%$ du coût complet actuel)

La production de Cloms pouvant être soit dirigée centralement par des appels à projets de cours auxquels répondent les équipes universitaires, soit émanant directement des universités qui distribueraient le financement selon les équipes en place et le contrat de site.

\section{Conclusions}

Nous sollicitons les autorités ministérielles pour développer oralement le 7 juin 2016 cette vision stratégique de licence ouverte plus avant et mettre en place les structures administratives permettant leur réalisation.

Le ministère oriente vers la Conférences des présidents d'universités françaises pour appel à projet, il apparaît que le terrain n'est pas encore prêt pour cette expérimentation en 2019. Ce bien que des mini-master soient créés outre-Atlantique et que la conférence européenne des Moocs indique une prise en charge par le répertoire national des certifications professionnelles en France. 


\section{La sortie du terrain : les valeurs de l'apprentissage}

Notre parcours de philosophe devenu anthropologue dans une industrie éducative nous a amené d'une entrée par le management et les activités de gestion (comptabilité, mercatique, gestion de projet) à une description de l'ingénierie au cœur de cette organisation : l'ingénierie de formation assurée par l'ethnie des ingénus avec une éthique synthétisant des arts (de la correspondance) et des métiers (les théâtres numériques comme la classe virtuelle) idoines.

Nous nous sommes efforcés, tout au long de ces descriptions ethnographiques, d'en abstraire des résultats anthropologiques plus généraux autour des notions de valeur, de temps ou encore de la sémiologie éducative. C'est pour cela que nous avons renoncé à une surcharge descriptive. Nous voulons prolonger cet effort d'abstraction dans ce chapitre en travaillant, à partir de notre matériau empirique, des domaines des sciences de l'éducation ayant trait à la théorie de l'activité, à celle de la transmission et enfin à la docimologie. La porte de sortie de notre terrain est ainsi celle de l'apprenant à distance qui expérimente les dispositifs produits ou, plus largement, des valeurs de l'apprentissage.

Nous commencerons ainsi par proposer un modèle de l'activité d'apprentissage présenté sous forme de schéma commenté. Il va du plus particulier (l'acte), au général (l'activité qui est répétitions régulières d'actes) auquel l'intention donne sens (c'est l'action intentionnelle des activités). Nous ne présenterons pas ici des développements récents du modèle autour de l'action polysémiotique, développement que nous devons à la contribution de Jean Marie Barbier. Le modèle premier et abstrait se concrétise par les observations que nous avons eues en tant que responsable d'une formation à distance en droit, complété par notre propre expérience d'apprenant à distance.

Puis nous nous centrons sur un dispositif d'évaluation en pleine évolution. Nous nous efforçons de décrire ce changement allant vers la profession professorale de ses pratiques, ou démonstration de son savoir faire et de ses compétences. Puis nous en tirons des conséquences à la fois pour l'apprenant (car il vise une bonne évaluation de ses apprentissages) et pour le formateur à distance (dont le métier se transforme). Cette évolution des évaluations nous permet enfin d'introduire des considérations plus générales sur l'éducation en termes de transmission et de relation éducative - avec la place particulière de l'apprentissage à distance qui réduit certaines distortions liées à la présence. 
Le dernier chapitre de cette section concerne la valorisation économique, plus que politique, de l'acte correctif. Nous décrivons et discutons le processus de valorisation de ce travail en industrie éducative et ses conséquences sur l'art de la notation. Nous constatons que l'économie contraint la docimologie dans une logique, là-aussi, de la métronomie du temps. Mais lorsque nous comparerons ceci au même processus de correction pour l'évaluation des diplômes supérieurs tels que le doctorat, nous observerons que la mesure de la valeur suit une logique de temps linéaire. De là, une perspective macrosociale émerge: l'évaluation numérique et quantitative est préférée pour la masse des étudiants alors qu'une évaluation nominale et qualitative est employée pour l'élite. Notre enquête qui demandait au départ "que vaut une formation", s'achève ainsi sur une évolution de la question vers "que vaut un étudiant ?" (voire un “élève"), déplaçant ainsi le centre de gravité du dispositif conçu par les ingénieurs vers l'apprenant lui-même. 
Nous savons que le Centre d'éducation à distance (Ced) est un établissement public administratif placé sous la double tutelle des ministères de l'Education nationale et de l'Enseignement supérieur et de la recherche. Cette institution est aussi connue sous le nom d' «académie en ligne » et propose des formations, pour le compte de l'État, depuis l'école primaire jusqu'au master.

La «Capacité en droit » est une formation proposée par le Ced en partenariat avec plusieurs universités, dont l'université Paris-1 Panthéon-Sorbonne comme entité fédératrice localement. C'est une préparation de deux années (capacité 1 et capacité 2 ) à un diplôme universitaire reconnu par l'État comme équivalent du baccalauréat. Institution ancienne des facultés de droit, issue de l'université impériale ou napoléonienne et post-révolutionnaire, c'est-à-dire faisant réaction aux grandes écoles de la première républqiue (1791) telles que pensées par Condorcet $^{62}$, la capacité en droit est souvent considérée comme un diplôme de la seconde chance, pour reprendre le chemin des études là où une scolarité secondaire tourmentée les avait dévoyées.

Nous allons décrire la formation «capacité en droit» avec une théorie de l'apprentissage distinguant actes, activité et action. Nous allons nous appuyer sur un double matériau empirique : d'une part notre propre parcours d'étudiant à distance ${ }^{63}$, d'une dizaine d'années antérieure à la date de rédaction de ce document; d'autre part notre position de terrain actuelle comme responsable de la formation «Capacité en droit» au sein du Ced. Nous avons donc un double point de vue : nous regardons le diplôme «par en-dessous » comme celui qui le prépare activement et «par en-dessus» comme celui qui le gère administrativement et pédagogiquement.

L'idée essentielle cadrant notre analyse est celle de méthode. Nous l'empruntons à Aristote qui évoque la «poursuite» (metà) d'un «chemin » (hodos) vers la connaissance pour translittérer les deux étymons grecs composant le mot. Filant la métaphore du chemin d'apprentissage, nous verrons que celui-ci se parcourt pas à pas, acte d'apprentissage après

\footnotetext{
${ }^{62}$ Nous faisons ici une brève référence à notre projet de livre: Marty O, Amirault R., 2020 (?) Nicolas de Condorcet and the French revolution of higher education, Springer. Key thinkers in education. Suisse. Ce projet de livre fait l'objet d'un contrat avec l'éditeur après acceptation par le directeur de collection.

${ }^{63}$ Formation à distance avec le Ced (Brevet d'État.) mais aussi avec le Service d'enseignement à distance (Sed) de l'université Toulouse le Mirail : Deug et licence de philosophie puis de sociologie.
} 
acte d'apprentissage. La marche régulière se fond alors dans une activité d'apprentissage avec son rythme et sa démarche propres. Enfin, l'ensemble du parcours devient une action d'apprentissage quand on lui donne du sens : c'est la direction du chemin vers sa destination finale (la reconnaissance collective du diplôme) qui permet de le comprendre comme une totalité unique. Actes, activité régulière et action sensée nous permettent ainsi de comprendre l'apprentissage avec la métaphore aristotélicienne du chemin : la méthode.

Cette théorie de la méthode, élaborée pour le séminaire doctoral du Cnam, peut-être schématisée comme ci-dessous. Nous allons ensuite colorier cette forme abstraite en l'appliquant à la réalité concrète et vivante de la capacité en droit préparée avec le Ced.

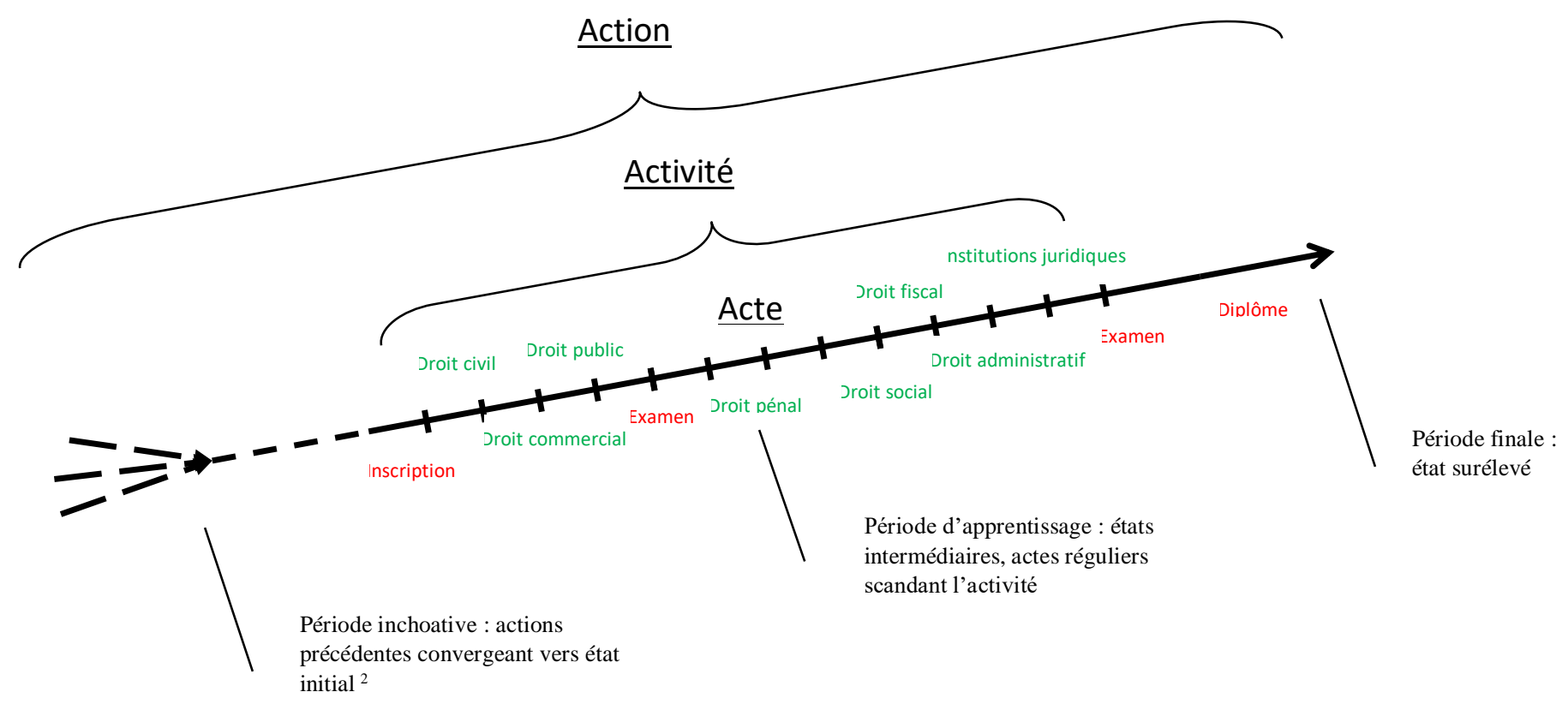

Le droit chemin : jeux d'échelles sur une méthode

\section{Apprendre le droit}

La capacité en droit, diplôme de la seconde chance, réunit en une même formation des étudiants de profils très variés : jeune lycéen de plus de dix-sept ans ayant échoué au baccalauréat, mère de famille voulant reprendre ses études, employé souhaitant passer un 
concours administratif pour progresser dans sa carrière... Des quatre cent inscrits en première année, seuls trente obtiendront le diplôme : la sélection est importante, plus importante que le taux de réussite à l'agrégation du secondaire portée par l'ethnie étudiée (quoique le niveau soit celui du master, ce qui réduit le nombre de candidats au concours). Ces heureux lauréats se ressemblent entre eux et à ce que l'institution attend d'eux : ils ont suivi le droit chemin - à la différence des non-reçus qui divergent et se fourvoient dans une multiplicité de parcours alternatifs expliquant l'échec. Nous prendrons donc comme modèle le « bon élève » ou élève normal qui suit le parcours de formation conformément à ce qui est attendu de lui. Quels sont ses actes? Quelle est son activité régulière? Quel est le sens de son action d'apprentissage ?

\section{Actes}

La méthode de la capacité en droit est un chemin qui se poursuit durant deux années : il est donc divisé en deux étapes. Chacune de ces années est elle-même fractionnée en semestres, selon le schéma européen. Et chaque semestre compte plusieurs matières. En première année, les étudiants doivent apprendre la méthodologie (comment lire les cours, rédiger une dissertation avec plan apparent, un commentaire d'arrêt), le droit civil, le droit commercial et le droit public. En deuxième année, ils ont le choix entre trois voies : la filière administrative, la filière judiciaire et la filière entreprise ; chacune de ces voies les amenant à étudier plus de quatre matières parmi les huit suivantes : droit commercial, droit administratif, droit fiscal, droit social, droit notarial, droit pénal, économie politique, institutions juridictionnelles et procès.

Le passage d'un module à un autre se fait librement dans l'année considérée. La progression se fait donc pas à pas, acte d'apprentissage après acte d'apprentissage. Chaque acte d'apprentissage permet de faire le pas, de progresser sur le chemin de la connaissance. Chaque acte est synonyme d'une compétence supplémentaire acquise : l'étudiant apprend à établir les faits, à les qualifier juridiquement et à les juger selon leur place dans la pyramide des lois ou la tradition de jurisprudence.

L'apprenant est donc actif : ce serait une mécompréhension profonde de le considérer comme inactif. Ses actes quotidiens se décrivent par des verbes d'action : écouter le DVD de cours, lire un chapitre des ouvrages fournis, consulter l'échéance des examens sur le site de téléformation, souligner pour fixer l'attention, rédiger une fiche de lecture, noter le vocabulaire dans un lexique juridique, réviser ce qui a été appris la veille, réciter les dates des lois et traités importants, rédiger un devoir ou cas pratique et, idéalement, discuter avec son 
entourage de ce qu'il vient d'apprendre et porter un regard juridique sur l'actualité. Ces actes se répètent quotidiennement, ils constituent l'activité de l'étudiant.

\section{Activité}

La difficulté que rencontrent les étudiants n'est pas tant dans l'accomplissement d'un acte isolé (tous ont au moins une fois ouvert les documents de cours et pris quelques notes) que dans la répétition régulière de ces actes. Il faut tenir la distance et maintenir l'apprentissage tout au long des deux années.

Ceci implique de pouvoir embrasser le programme de l'année pour diviser la totalité impressionnante de cours adressés au premier semestre (plus de trois kilogrammes de livres et fascicules, une dizaine de devoirs à envoyer à la correction) en parties assimilables et à les répartir le long d'un programme d'apprentissage individuel. L'organisation de son travail est donc d'abord annuelle (Dans quel ordre apprendre les matières ? Quel mois vais-je commencer les révisions en vue de l'examen ?), puis hebdomadaire (Quels sont les jours de la semaine que je vais consacrer à l'étude ? Quel temps me permet de dégager ma profession ou toute autre contrainte extérieure ?) et enfin quotidienne (Quel est le meilleur moment de la journée pour apprendre? Combien d'heures puis-je rester concentré ?). Ainsi l'activité d'apprentissage est-elle descriptible selon différentes échelles de temps.

L'étudiant doit aussi combiner sa vie sociale (équilibre des sorties, de la famille et des amis qui doivent accepter cette nouvelle activité) et sa vie physiologique (nourriture saine pour soutenir l'effort, temps de repos nécessaires à l'assimilation) pour optimiser son apprentissage. Chaque jour il se met seul au travail, sans ajournement ou longs préparatifs, selon des habitudes et des rituels qu'il se donne à lui-même. Il envoie périodiquement des devoirs à la correction pour trouver des repères et marquer le passage entre les matières : il avance pas à pas en s'assurant de l'un avant de passer à l'autre. L'étudiant est capable, enfin, de faire varier son activité selon les périodes d'examen finaux. C'est le moment des relectures, révision et récitations où tout est refait afin d'être prêt pour l'évaluation finale. C'est ce qui donne sens à son activité. Il s'inscrit là dans une logique plus globale qui donne une fin à son action d'apprenant.

\section{Action}

Le chemin d'apprentissage, ou méthode, arrive à son terme lorsque l'étudiant passe l'examen final et obtient son diplôme. C'est donc un chemin qui a un sens, il est orienté 
depuis le point de départ jusqu'au point d'arrivée : l'apprenant le parcourt les yeux tendus vers l'objectif final. C'est ce sens qui transforme l'activité en action. L'action suit une direction fléchée et n'est pas simple succession chaotique d'actes, elle n'est pas agitation ni activisme de façade.

L'action sensée donne une unité à l'apprentissage: l'étudiant construit progressivement son identité de capacitaire en droit et cette identité se parachève lorsqu'il obtient le diplôme. Le diplôme, tout insécable qui donne sens aux partiels, s'apparente à un rite de passage qui change l'état social de l'étudiant : mis à l'écart avec ses pairs le temps de l'épreuve, il en ressort transformé, métamorphosé. Il était «capacitaire », il devient «lauréat», en capacité de «faire son droit»: il peut s'inscrire en première année universitaire (voire directement en deuxième année pour les plus adroits qui obtiennent une moyenne supérieure à quinze sur vingt), passer un concours administratif de catégorie B, évoluer dans son entreprise ou son administration avec la reconnaissance sociale de ce diplôme.

Tout ne se joue cependant pas le jour de la remise du diplôme. Celle-ci n'est jamais qu'un couronnement, cérémonie formelle préparée par un travail de fond qui a duré deux années. Les actes réguliers sont soudainement légitimés par l'arrivée à l'étape ultime d'apprentissage, ils avaient pourtant déjà un sens tout au long du chemin. Ainsi, c'est progressivement que l'étudiant a appris une logique (celle des réalités décrites dans les différentes matières, avec le vocabulaire spécifique noté dans son lexique) et une éthique (un comportement fondé sur une mémoire collective du droit et de ses valeurs déontologiques ; un imaginaire partagé quant aux futurs rôles professionnels et les costumes qui devront y être endossés) qui font de lui un juriste. En suivant son parcours d'apprentissage dans le bon sens, il a acquis un sens commun juridique (l'implicite infra-logique qui tombe sous le sens de tout homme de droit) et un sens particulier (éthique individuelle qui le situe comme élève unique par rapport à sa discipline, avec son propre projet idiosyncratique). À l'issu de ces deux années, l'étudiant, en capacité de «faire son droit», doit avoir profondément modifié ses habitudes : il porte des habits appropriés, habite la ville en juriste en fréquentant les bonnes institutions. Il a appris à vivre pleinement le droit : il sent, il voit, il pense, il dit les formes juridiques de tout fait social. En un mot, il a assimilé une culture d'action ${ }^{64}$ juridique.

\footnotetext{
${ }^{64}$ Barbier JM, "Cultures d'action et modes partagés d'organisation des constructions de sens ", Revue d'anthropologie des connaissances $1 / 2010$ (Vol 4, n 1), p. 163-194.
} 
Osons ici un parallèle. Un grand homme de théâtre se voit hors des théâtres : il repère instinctivement, dans toute situation sociale, la mise en scène, l'acte, le costume et le bon mot qui produiront un effet spectaculaire. De même, le juriste abouti se montre tel après la dernière étape du chemin d'apprentissage: ses mœurs sont droites, il vit le droit spontanément. Ainsi, il cite un arrêt célèbre pour alimenter son opinion quant à l'actualité, sans réciter fastidieusement le cas en son entier comme il le faisait lorsqu'il était étudiant. Sa compétence est de granularité fine et peut nourrir les bouches les plus délicates.

\section{$\underline{\text { Apprendre à distance }}$}

Jusqu'à présent, nous avons décrit un apprentissage en droit tel qu'il pourrait être mené en présence du professeur donnant ses cours en amphithéâtre de faculté. De fait, la capacité en droit est aussi délivrée par des universités, ce sont même des partenaires universitaires qui confèrent le statut étudiant, le diplôme final et qui organisent certains regroupements. Le Ced offre, pour sa part, la possibilité technique d'apprendre à distance et une partie du corps professoral en charge, notamment, des corrections. Centrons-nous maintenant sur les particularités de la méthode du Ced : l'apprentissage à distance et sa pédagogie propre. Étudions ce qui, dans l'art d'apprendre, se conserve, ou au contraire évolue, avec les techniques de la distance.

Actes

Parmi les actes d'apprentissage propres à la capacité en droit avec le Ced, l'étudiant doit savoir surmonter la distance, c'est-à-dire se rapprocher de l'institution en cas de difficulté. Il doit apprendre à envoyer un document selon les formes demandées (modèle de copies à adresser à la correction et circuit de distribution particulier, utilisation du logiciel Copie en ligne ou envoi postal), accéder au tutorat qui se trouve sur le site Internet de la formation pour demander de l'aide, voire téléphoner à un conseiller qui pourra l'orienter dans l'organisation de son travail. Le premier acte de l'apprentissage à distance est donc l'apprentissage de l'interaction avec l'institution.

Le deuxième acte de l'apprentissage à distance consiste lui-aussi à rompre l'isolement, non pas pour demander de l'aide auprès de ses professeurs, mais pour s'insérer dans le collectif des étudiants. Ce sont les regroupements en présentiel et les échanges sur le forum du 
site Internet qui recréent l'émulation et la dynamique collective d'une salle de classe. C'est là que s'apprennent les formes des activités informelles : accent et modes vestimentaires, loisirs communs, références estudiantines et jugements partagés sur l'expérience d'apprentissage. Ce deuxième acte est celui qui crée l'esprit d'une promotion, son homogénéité et la solidarité qui fera sa force ultérieure.

Les interactions distantes avec le corps professoral et avec la promotion permettent de s'ajuster progressivement, d'être corrigé, pour se fondre dans la masse et suivre, ensemble, le même chemin. Cet ajustement doit être régulier, il est ainsi une activité à part entière.

\section{Activité}

La régulation de l'activité par les rétroactions professorales (devoirs corrigés, conseils prodigués sur le forum) guide les étudiants sur le droit chemin. Celui-ci se parcourt à son rythme, avec une démarche propre et selon les contraintes individuelles d'emploi du temps. La distance offre en effet plus de liberté aux apprenants qui peuvent se former en parallèle de leur travail, lors de leurs moments de loisir. La scolarité se rapproche alors du sens classique de la skholê grecque, c'est-à-dire du temps de loisir sagement occupé. L'apprentissage n'est plus réservé à une classe de loisir ${ }^{65}$ ayant l'oisiveté des privilégiées : elle est accessible à tous.

Liberté et égalité face à la formation à distance ne signifient pas pour autant anarchie et autodidaxie : le pouvoir du maître subsiste et les apprenants ne sont pas livrés à euxmêmes. Le Ced reste un tuteur rigide qui guide les étudiants et les fait pousser droit pour qu'ils s'élèvent au plus haut de leurs capacités. Toutefois, aucun docte jardinier n'est là pour redresser les natures broussailleuses par la parole. L'élève doit s'accrocher seul aux écrits qui lui sont envoyés. Son autonomie consiste à suivre de lui-même et régulièrement les normes d'apprentissage de l'institution qui sont omniprésentes.

La distance parcourue tout au long de la formation est une course de fond. L'apprenant doit faire preuve de volonté et de ténacité dans son travail. Le philosophe Alain, une décennie avant la création du Ced en 1939, énumérait les qualités de celui qui sait se gouverner dans ses études: l'assiduité (continuité dans le temps), l'exactitude (commencer et arrêter à l'heure : la ponctualité), la mise en train (pas d'ajournement ou de longs préparatifs), l'ordre (une chose à la fois), le repos (dormir, oublier), la règle (se donner un emploi du temps), la patience (ténacité, suite). Ce travail et cette volonté dans leur activité d'apprentissage sont

\footnotetext{
${ }^{65}$ Veblen T., 1979, Théorie de la classe de loisir, Gallimard. Tel. France.
} 
communs aux étudiants qui réussissent à distance, quel que soit leur profil individuel ; ils leur permettent de construire une identité remarquable leur apprenant à faire face aux situations les plus complexes 66

\section{Action}

Actes variés d'interaction avec l'institution, activité libre mais régulière, l'apprentissage à distance devient une action lorsqu'elle prend sens dans une volonté à long terme de se former tout au long de la vie, que ce soit pour s'adapter à son poste de travail (travailler le soir, en complément de son emploi) ou pour se développer personnellement (éducation permanente, culture générale). Loin d'un sprint où l'étudiant ingurgiterait précipitamment des quantités d'écrits pour les régurgiter sur sa copie le jour de l'examen (pratique répugnante pour les enseignants), les étudiants à distance sont en promenade permanente sur les chemins de l'apprentissage : chaque jour leur esprit cueille et goûte des savoirs nouveaux comme une bouche déguste des saveurs exotiques. Le plaisir d'apprendre, l'envie de progresser et un appétit régulier pour des connaissances variées permettent à l'étudiant d'assimiler sans cesse, de digérer et d'incorporer les nourritures de l'âme. Son action devient existentielle : il vit de et pour l'apprentissage.

L'évaluation, dans cette voie, n'est alors qu'une formalité de passage sur les chemins de l'apprentissage pour s'assurer que la démarche est la bonne. Elle permet de fixer un objectif avec sa temporalité propre (par exemple l'étudiant se donne deux années pour apprendre les bases droit) et donne un repère quant aux acquis de l'étape. De même qu'un passeport est tamponné lors d'une escale sur une route internationale, le visa du contrôle permet de faire valoir le parcours lorsque l'on veut passer à une autre étape (entamer une licence de droit sans le baccalauréat par exemple). Et pour l'étudiant qui veut parcourir beaucoup de chemin, cela lui donne repère personnel : il sait par exemple, qu'il a acquis tel niveau dans telle discipline, à tel moment de sa vie (obtenir la capacité en droit pour, quelques années plus tard ensuite s'inscrire à la faculté d'économie).

Ces profils d'étudiants polymathes du Ced, plus rares, se construisent progressivement, au fil des expériences de formation réussies. Ce sont ceux qui empruntent plusieurs chemins d'apprentissage : révision en mathématiques pour assoir ses bases, modules libres de latin par curiosité intellectuelle, formation au droit pour prendre de la distance avec

\footnotetext{
${ }^{66}$ Alain, 1986 (1932), Propos sur l'éducation - Pédagogie enfantine, PUF. Quadrige Grands Textes. Paris. 19 ème leçon "La volonté et le travail », p. 305. Alain écrit : "Ce sont les hommes qui dans leurs études ont su se gouverner, et par cela seul ils sont et restent supérieurs, quelle que soit la tâche. ».
} 
son parcours professionnel dans le monde de la justice, module de bureautique pour mieux rédiger avec un logiciel de traitement de texte ${ }^{67} \ldots$ Ces inscrits, grâce à la flexibilité de l'apprentissage à distance, vivent au quotidien la politique européenne de formation tout au long de la vie. Ils vont sur le droit chemin.

Ceci nous amène à nous interroger sur l'évaluation de ces inscrits allant dans le bon sens. En montrant l'évolution des évaluations, on verra que certaines valeurs éducatives se transforment.

${ }^{67}$ Formations à la bureautique où l'interactivité se fait avec le didacticiel et non plus avec un enseignant humain : action et rétroactions formatives sont pour moitié automatisées, l'apprenant est cadré par la machine et doit en épouser les formes. 


\section{De l'évaluation des épreuves aux valeurs éducatives}

\section{$\underline{\text { Introduction }}$}

Nous voulons ici mener une analyse anthropologique (laissant une large place de référence aux textes philosophiques) des activités d'apprentissage et de leur évaluation en dégageant les conséquences d'une évolution réglementaire des modalités de concours, tant pour les sciences de l'apprendre que pour la didactique. Nous nous placerons ainsi d'abord du point de vue de l'apprenant (partie 1), puis du point de vue du formateur (partie 2), pour voir en quoi une nouvelle norme d'évaluation modifie leurs pratiques. Nous procéderons ensuite à une ouverture critique de ce nouveau système éducatif (partie 3) avant de synthétiser les deux points de vue dans la notion de relation éducative entre apprenant et formateur (partie 4).

\section{Définition}

Définissons tout d'abord la notion de norme. Là où plusieurs voient dans le mot « norme » l'idée d'un comportement moyen (au sens statistique d'une loi normale), ou encore d'une règle droite à suivre, nous choisissons de retenir le sens de nomos ${ }^{68}$, c'est-à-dire de tradition ou de coutume. Nous étudierons alors le nouveau type d'évaluation dans la perspective de rupture innovante avec la tradition, de nouvelle norme formatant les pratiques.

\section{Données empiriques}

Nous observons que le recrutement des enseignants du second degré évolue vers un nouveau type d'épreuve, consistant non pas seulement en une composition technique dans la discipline visée (carte de géographie, thème de grec ancien, version d'espagnol, commentaire d'arts plastiques...) mais en une description de ses pratiques pour mettre en valeur les

\footnotetext{
${ }^{68}$ Nous reprenons la distinction étymologique d'Hayek, dans Droit, législation et liberté, entre nomos (droit traditionnel) et tesis (droit voté à l'assemblée).
} 
compétences déjà acquises. Ainsi la reconnaissance des acquis de l'expérience (Raep ${ }^{69}$ ) permet aux candidats aux concours internes du Capes et du Crpe de synthétiser dans un dossier leurs pratiques et d'en faire profession devant le jury les examinant. Ils professent alors leur profession de professeur, avec toutes les compétences qui font la professionnalité du métier. De même le dossier numérique de compétences (Dnc) du certificat informatique et internet de deuxième niveau pour enseignants $(\mathrm{C} 2 \mathrm{I} 2 \mathrm{e})$ est un condensé des preuves pratiques que les candidats sont en mesure d'exercer la profession dans ses dimensions informatiques. Cette norme d'évaluation est d'ailleurs présente dans l'enseignement supérieur et la recherche puisqu'elle existe déjà pour le recrutement des maîtres de conférences (Mcf) et professeurs des universités $(\mathrm{Pu})$ comme des ingénieurs et techniciens de recherche et de formation (Itrf) : dans chaque cas un dossier est demandé et le candidat doit y apporter les preuves pratiques de ses compétences (notamment les projets de recherche pour les premiers, le travail d'ingénierie pour les seconds). L'évolution de la norme d'évaluation ne concerne donc pas nécessairement toujours le support (réunir toutes les pièces sur dans un dossier informatisé) mais bien l'objet : il s'agit d'évaluer les pratiques attestant de la capacité à exercer le métier visé.

Cette norme d'évaluation n'est pas neutre, elle relance la tension entre savoirs d'action (le savoir-faire acquis par la pratique) et savoirs théoriques (traditionnellement appris à l'école et examiné lors de la composition d'un concours) déjà abondamment travaillée lors d'un séminaire du Centre de recherche sur la formation animé par Jean Marie Barbier ${ }^{70}$. Fautil évaluer ce que le candidat a déjà fait et peut à tout moment refaire, sa connaissance pratique du métier, ou au contraire évaluer les descriptions du métier que le candidat a entendues par des professeurs et qu'il énonce à son tour? L'histoire des normes d'évaluation semble aller vers la première des deux hypothèses.

\section{$\underline{1 / \text { Conséquences de la Raep pour l'apprenant }}$}

$\mathrm{Au}$ début du $20^{\text {ème }}$ siècle, le psychologue Gustave Lebon tentait de réformer l'université et il écrivait «Professeurs, parents et élèves dédaignent absolument ce qui n'est

\footnotetext{
${ }^{69}$ Cette épreuve s'inscrit dans la loi de modernisation de la fonction publique du 2 février 2007, comme le souligne Yolande Ferrandis dans La Raep dans les concours, La documentation française (2013).

${ }^{70}$ Barbier JM (dir.), 2011, Savoirs théoriques et savoirs d'action, PUF. Éducation et Formation. France.
} 
pas matière à examen $»^{71}$ (Lebon, 1920, p. 180). Il déplorait en effet que le jugement, la raison, l'observation ne fussent pas développés à l'université ou l'apprentissage par cœur et la mémoire restaient maître. Il expliquait cela par des techniques d'évaluation basées sur la récitation du cours qui n'incitaient les élèves qu'au bachotage sans compréhension ni jugement.

La réflexion du docteur Gustave Lebon est toujours d'actualité dans le sens où le mode d'évaluation est déterminant quant à ce que les élèves vont réellement apprendre et les professeurs réellement enseigner : c'est en effet l'épreuve de vérité où tout le programme se joue et chacun, par souci d'efficacité, centre donc son attention et ses stratégies d'apprentissage sur les modalités d'évaluation.

Changer le mode d'évaluation - la preuve de l'apprentissage - est donc plus important que changer les programmes - ce qui doit être appris. C'est en effet changer les qualités et aptitudes que l'apprenant va développer en vue de réussir l'évaluation, et ce quel que soit le programme enseigné. La généralisation du dossier numérique de compétences ou encore de la reconnaissance des acquis de l'expérience n'est donc pas anodine : c'est une pratique qui va cadrer les apprentissages. Un effet gigogne est à souligner : les enseignants ainsi recrutés vont eux-mêmes développer ces techniques d'évaluation (pour le brevet informatique et internet (B2I) par exemple) pour former leurs élèves.

Quels sont les changements dans les techniques d'apprentissage qui vont découler de cette nouvelle norme d'évaluation? La première et la plus évidente est le développement de la réflexion personnelle. Celui qui doit aller puiser dans ses pratiques ce qui correspond aux compétences attendues, qui doit formaliser ce qu'il a fait plus que se former à de nouveaux énoncés, va nécessairement se décentrer pour réfléchir sur lui-même - en prenant soi-même comme un autre, l'individu reste, certes, centré sur lui-même, mais il effectue un décentrement réflexif. Nous pouvons ici citer les travaux menés sur les e-portfolio qui développent cette nécessité de la distance réflexive ${ }^{72}$. Il doit alors apprendre à se regarder au miroir des compétences attendues dans le référentiel national (un référentiel de sept domaines de compétences pour le C2I2E par exemple). Ce type d'évaluation encourage donc les modes

\footnotetext{
${ }^{71}$ Lebon G., 1920, Psychologie de l'éducation, Édition Kindle de Flammarion, emplacement 184/5006.

72 L'analyse de la reconnaissance des acquis de l'expérience par un dossier numérique de compétences s'apparente à la littérature scientifique sur les e-portfolio. Notamment : Lopez-Fernandez et Rodriguez-Illera, 2008, "Investigating university students' adaptation to a digital learner course portfolio » in Computers \& Education $\mathrm{n}^{\circ} 52$ (pp608-616) ou encore Cheng, Chau (2012) « A study of the effects of goal orientation on the reflective ability of electronic portfolio users " in Internet and Higher Education $n^{\circ} 16,(p p 51-56)$
} 
d'apprentissage de praticiens réflexifs, qui s'exercent et analysent leurs actions sans cesse pour pouvoir les professer utilement le jour de l'évaluation - c'est-à-dire dans le cadre du référentiel de compétences qui leur est imposé par l'épreuve.

Une deuxième conséquence est de développer, non pas la faculté de concevoir et d'énoncer des choses abstraites, comme dans l'évaluation classique par dissertation, mais plutôt la faculté de rester malléable pour se former sans cesse par la pratique. L'apprenant développe la volonté de se laisser faire, de se désinhiber: il se laisse aller, se laisse transformer par ses actes et prend acte de ces transformations en notant ce qu'il apprend. La recherche de pratiques variées maintient alors la polymorphie juvénile et permet de garder la forme, c'est-à-dire la possibilité de se former sans cesse en changeant de forme.

L'apprenant, dans cet exercice de profession de ses pratiques (ou profession de soi) apprend à mettre en forme le fond de son expérience, il formalise sans cesse ses actions. Cette mise en forme n'est pas superficielle : il apprend à faire émerger à la surface le fond de ses compétences accumulées dans les profondeurs de son expérience. La profession de soi, le fait de retrouver dans ses pratiques ce qui correspond aux compétences attendues, est un travail sur soi pour formaliser ce que l'on est selon les formes attendues par le jury. L'apprenant doit trouver dans la panoplie de ses costumes passés l'uniforme conforme à la forme demandée.

Si l'on considère à présent cet exercice de profession de ses pratiques à la lumière de la dialectique entre savoirs d'action et savoir théoriques développée par Jean Marie Barbier ${ }^{73}$, on peut rappeler que les savoirs théoriques sont plus riches sémantiquement (vocabulaire plus varié et moins polysémique que pour les savoirs d'action) et plus intelligents au sens où ils lient sans cesse les faits les uns aux autres (alors que les injonctions des savoirs d'action ne prennent pas le temps de relier les faits par le verbe). La profession de ses pratiques appelle donc, en plus de la compétence réflexive, le développement de la faculté d'énonciation de la part des apprenants qui sont conduits à questionner ce qu'ils font pour le verbaliser consciemment. Ils vont et viennent entre le général correspondant à la compétence attendue et le particulier qui est l'exemple de vécu qui prouve qu'ils ont la compétence.

Les facultés que doit développer l'apprenant avec cette nouvelle norme d'évaluation et que nous venons de détailler peuvent être résumées dans la liste ci-dessous :

\footnotetext{
${ }^{73}$ Barbier JM (dir.), 2011, Savoirs théoriques et savoirs d'action, PUF, Éducation et Formation. France.
} 
1. réflexion personnelle,

2. pratiques variées,

3. formalisation de ses activités,

4. verbalisation intelligente.

Examinons à présent les conséquences de la profession de ses pratiques sur les techniques d'enseignement, sur la didactique. En quoi le métier de formateur est-il affecté par ces nouvelles normes d'évaluation?

\section{2/ Conséquences de la Raep pour le formateur}

Pour désigner les pratiques didactiques que nous allons décrire, nous reprenons la distinction antique entre pédagogie et méthode, modernisée par la dichotomie entre éducation et enseignement. Nous avons élaboré un tableau à double entrée permettant de visualiser ces types didactiques. Les deux dimensions sont la méthode et la pédagogie, elles permettent de repérer quatre types de pratiques : l'éducation (essentiellement pédagogique), l'enseignement (méthodique de nature), l'art oratoire (qui est une didactique faible) et le professorat ou formation (une didactique forte, c'est-à-dire à la fois pédagogique et méthodique). Nous appuyons ce modèle sur la théorie de la distance transactionnelle de M.G. Moore ${ }^{74}$ qui croise l'axe de la structure (ici méthode) et du dialogue (ici pédagogie) dans un espace bidimensionnel.

74 Moore Michael Grahame, 2007, "The Theory of Transactional Distance" in Handbook of Distance Education, pp. 89-105, Second Edition, Edited by Michael Grahame Moore, Lawrence Erlabum Associates, publishers, Mahwah, New Jersey, London. Nous avons traduit, avec l'accord de l'auteur, ce chapitre qui est archivé sur HalShs : http://halshs.archives-ouvertes.fr/halshs-00777034 


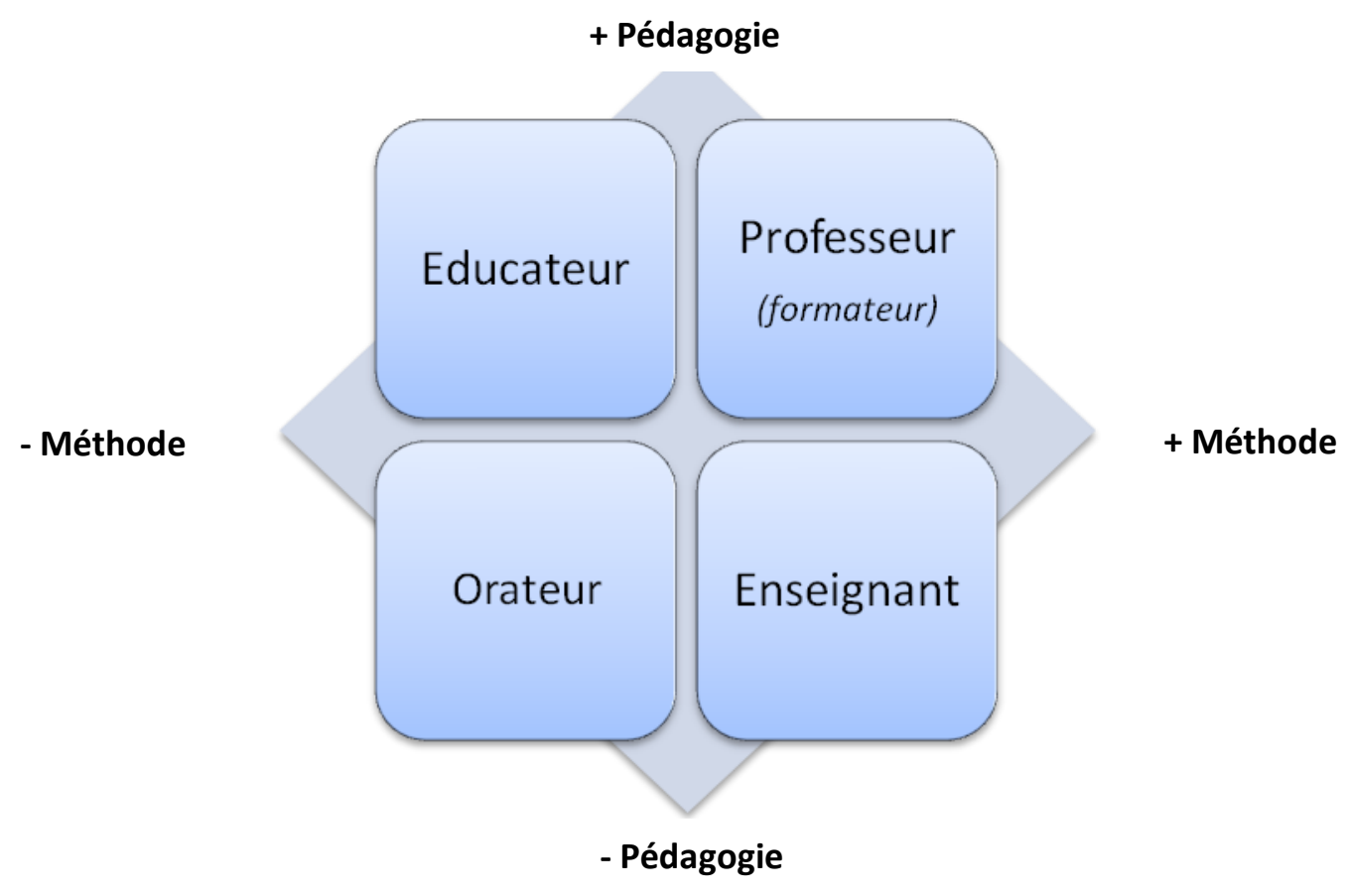

\section{Types didactiques}

Les mots désignant ces types didactiques ont été choisis selon leur étymologie, en partant du principe que le sens premier gardait encore un pouvoir de signification aujourd'hui, parmi les multiples acceptions modernes et contradictoires de ces termes. Ainsi la « pédagogie », construit sur deux étymons grecs, consisterait à «mener » les plus «jeunes », et ainsi définirait «l'éducation », au sens latin premier d'accompagnement et de conduite. Cet axe vertical est croisé, dans notre tableau, avec la «méthode », terme aussi composé de deux étymons grecs et qui rappelle le «chemin» parcouru vers la «connaissance » (comme le traduit l'expression anglaise en vogue de learning path), ce qui est le propre de « l'enseignement » au sens large de « signe » de connaissance à transmettre.

Dans notre matrice, l'éducation est du côté de la pédagogie alors que l'enseignement est de celui-de la méthode. L'opposition majeure enseignant-éducateur différencie les pratiques didactiques centrées sur la transmission de connaissances (enseignement) et celles qui privilégient l'accompagnement et la relation maître-élève (éducation) : d'un côté les enseignant méthodiques, d'un autre les éducateurs pédagogues. 
Nous ajoutons à cette opposition matricielle deux autres types didactiques : tout d'abord l'orateur qui est celui qui dit devant un auditoire. Nous critiquons cette posture qui néglige pédagogie et méthode : l'orateur, parle devant un auditoire sans souci de ce qui est dit et de ceux à qui cela est dit, légitimé par sa position sur la tribune. Cet orateur-là n'a pas la professionnalité qui fait la profession de professeur - type didactique fort dans notre modèle.

Le professeur est un type didactique fort au sens de formateur : c'est le médiateur qui fait passer au mieux ses idées à ses élèves, pour les transformer. Le formateur est ainsi celui qui modèle les formés, il transforme leur esprit et leur vision pour les amener à voir les bonnes formes ${ }^{75}$. Nous nous appuyons ici sur le travail de Michel Fabre reprenant La physique d'Aristote : ainsi la réalité prendra les mêmes formes dans leur esprit que dans celui du formateur, ils partageront les mêmes idées (au sens d'eidos). Il n'a pas face à lui des étudiants informes (ou amorphes) mais difformes, qu'il va transformer (métamorphoser) vers une forme idéale. Malgré le travail de conformation de la promotion de formés, le résultat ne sera jamais uniforme mais au contraire multiforme car tous les formés sont différents et la forme obtenue jamais pure.

Cette typologie nous permet de mieux comprendre l'impact de la norme d'évaluation de profession de ses pratiques sur la didactique. Les épreuves considérées servent à évaluer de futurs professeurs. Il s'agit donc de former les professeurs - qui deviendront à leur tour formateur lorsqu'ils atteindront le sommet de leur art. Et cette formation passe par l'enseignement d'un contenu et une éducation pour les accompagner dans leur apprentissage.

En matière d'enseignement, nous pouvons prendre l'exemple du dossier numérique de compétences dans le dispositif C2I2e. Le référentiel est ici public : les compétences attendues de la part des candidats à la certification sont données publiquement sur un site Internet (http://www.c2i.education.fr/spip.php?article87 ${ }^{76}$ ). Libre à chacun de mettre en œuvre les moyens de les acquérir et de les exprimer correctement dans un dossier approprié qu'évaluera le jury. Le contenu est donc connu (les compétences à obtenir), il est supposé acquis (durant le passé du préparationnaire) et s'il ne l'est pas les organismes de formation aident à l'acquérir (par des exercices d'enseignement utilisant les technologies de l'information appliquées à l'éducation). Le contenu, ou méthode, est donc dans ce modèle donné. Le

\footnotetext{
${ }^{75}$ Fabre Michel, 1994, Penser la formation, PUF, l'éducateur. France. Nous nous appuyons sur le chapitre concernant la lecture de la Physique d'Aristote par Heidegger.

${ }^{76}$ Article consulté le 17 février 2013 sur le site national dans C2I2E.
} 
cheminement est gratuit, tout au plus le formateur vendra-t-il une aide à la démarche réflexive, un accompagnement.

Et l'on entre ici dans le domaine de l'éducation. Pour préparer le futur professeur, le formateur l'accompagne et le conduit sur le chemin qu'il a à suivre. Il l'aide à se décentrer pour prendre une distance réflexive avec ses pratiques pour les formaliser sous forme de récit d'expérience dans le dossier numérique de compétences. En plus de ce rôle principal, le formateur fait œuvre d'éducateur en accueillant l'aspirant professeur, en faisant un bilan avec lui des compétences manquantes et en l'orientant dans le chemin à suivre pour acquérir ces expériences manquantes. Il le secourt en cas de situation difficile et enfin reconnaît le chemin parcouru en l'aidant à le formaliser.

La norme d'évaluation de la profession de ses pratiques implique donc une double évolution du métier de formateur et un modèle économique particulier. Le formateur aide l'apprenant à acquérir du contenu (la méthode est gratuite) et l'accompagne dans sa démarche réflexive (la pédagogie est payante). On se rapproche d'une technique d'enseignement ici aussi corporatiste: celle du compagnonnage médiéval. La relation éducative prime sur l'enseignement : la hiérarchie entre le maître et l'apprenti (le formateur et le futur professeur) est importante.

Nous avons ainsi décrit scientifiquement ce qui était visible sur le terrain et analysé philosophiquement comment l'évolution de l'évaluation des enseignants du second degré entraînait deux types de conséquences : d'une part sur les sciences de l'apprendre avec le développement de la réflexion personnelle, de pratiques variées, de verbalisation de ses activités, de formalisation de son expérience ; et d'autre part sur la didactique avec le primat de l'éducation sur l'enseignement, de l'accompagnement sur l'apprentissage pur. Tout n'est cependant pas positif. Cette norme d'évaluation par profession de ses pratiques est en effet critiquable dans le sens où elle ne laisse pas de chance à celui qui, faute d'être du bon milieu, n'a jamais connu le métier, n'a pas accompli les bonnes activités à formaliser pour l'évaluation ${ }^{77}$.

Perspective critique sur la question de l'égalité dans cette nouvelle légalité

\footnotetext{
${ }^{77}$ C'est pour cela que la politique du stage, à visée formative, est inséparable de l'évaluation par profession de ses pratiques.
} 
Du fait de cette nécessité d'avoir pratiqué pour pouvoir être reconnu comme apte à enseigner, l'idéal méritocratique de la Révolution française serait perdu et l'on risquerait un retour des corporatismes : seuls les héritiers ${ }^{78}$ pourraient professer leurs pratiques car, fils de praticiens, ils ont appris à faire et peuvent y réfléchir. La logique de la reproduction des classes sociales que dénonçait le sociologue Pierre Bourdieu ${ }^{79}$ serait ici aussi implacable. Le mythe du concours reste faussé : tous les concurrents courent certes vers la même ligne d'arrivée, mais chacun a son point de départ, selon son milieu social ou l'institution qui l'a préparé et l'a positionné différemment au moment de s'élancer. Quant au classement sur la ligne d'arrivée, il renseigne peu ou pas sur l'état d'arrivée : il ne dit rien de l'épuisement de ceux qui viennent de loin et ont beaucoup couru (le chemin est long depuis les milieux défavorisés) ou même de la réorientation de certains pour qui c'était trop facile et qui ne se sentent pas concernés par le devenir attendu des vainqueurs. En résumé, la profession de soi comme mode d'évaluation change certes les techniques d'apprentissage, mais elle ne résout en rien la contradiction de notre société entre le mythe égalitaire du concours et la réalité des inégalités éducatives et d'accès à un métier.

Le philosophe de l'éducation John Dewey ${ }^{80}$ rappelle la fonction sociale de l'école qui est de transmettre à la nouvelle génération le meilleur de ce que l'ancienne génération a connu - nous nous référons ici à son chapitre introductif de Democracy and Education et non aux vues constructivistes avant l'heure développées dans Experience and Education. L'expérience des anciens, traduite en signes verbaux, est léguée aux plus jeunes. Ce sont à la fois une vision de la réalité et un ethos (gestes, comportements, valeurs, normes, règles, émotions,...) qui sont transmis. C'est la mémoire collective qui passe d'une génération à une autre, qu'elle soit mémoire vive, transmise par la parole, ou mémoire morte qui se fixe dans les écrits et dispositifs techniques - la distance privilégiant la seconde, mémoire suffisamment dure pour durer.

Nous croisons cette idée à nouveau avec la sociologie de l'éducation de Pierre Bourdieu $^{81}$, montrant la proximité culturelle des classes dominante avec la culture scolaire : la culture cultivée est plus proche des savoirs légitimes qui s'enseignent à l'école que les cultures populaires - qu'elles soient étrangères (immigration) ou ouvrières. Pour illustrer ceci, nous pouvons songer au concours d'entrée à l'Institut d'Études Politiques, où la reproduction

\footnotetext{
${ }^{78}$ Au sens premier espagnol de hijo de algo, fils d'un noble possédant un titre

${ }^{79}$ Bourdieu P., 1970, La reproduction, Éditions de Minuit. Collection le sens commun. Paris.

${ }^{80}$ Dewey J., 2004, Democracy and education, Dover Publication.

${ }^{81}$ Bourdieu P., 1970, La reproduction, Les éditions de Minuit. Paris.
} 
sociale est très forte et où l'épreuve de dissertation de «culture générale » traduit l'appartenance à la bonne culture. Toutefois, cette épreuve est jugée inutile dans le cas de dossiers scolaires de très bonne qualité qui sont admis sans passer le test de culture générale.

Croisant les théories de Bourdieu et de Dewey, nous obtenons ainsi un modèle de trois cercles concentriques, plaçant la culture scolaire au centre, entourée de la culture dominante, puis des cultures populaires périphériques. Cette vision d'un centre culturel à transmettre montre comment chaque société fait germer dans l'esprit de la nouvelle génération le noyau culturel qui lui permettra de reproduire le meilleur de ce qu'elle a été.

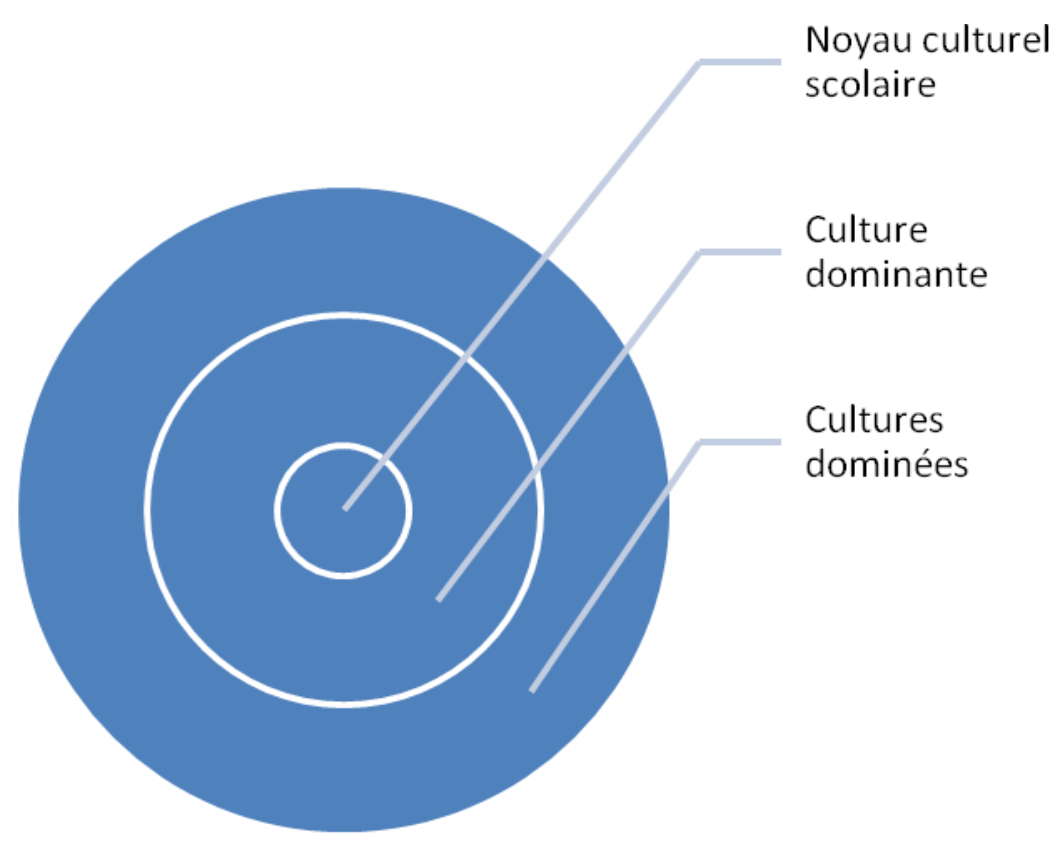

\section{Transmission du noyau culturel par l'école}

Ce qui peut être détaillé par le schéma suivant, représentant spatialement une typologie des savoirs selon qu'ils sont plus ou moins centraux ou périphériques dans notre culture ${ }^{82}$ :

\footnotetext{
${ }^{82}$ Nous sommes conscients que nous prenons ici le risque de "l'espitémocentrsime scolastique " dénoncé par Pierre Bourdieu dans ses Méditations pascaliennes. Nous soutenons la vision d’Henri Mendras, dans La seconde révolution française, d’une " galaxie centrale » que nous présentons hiérarchisée dans les schéma ci-dessous.
} 


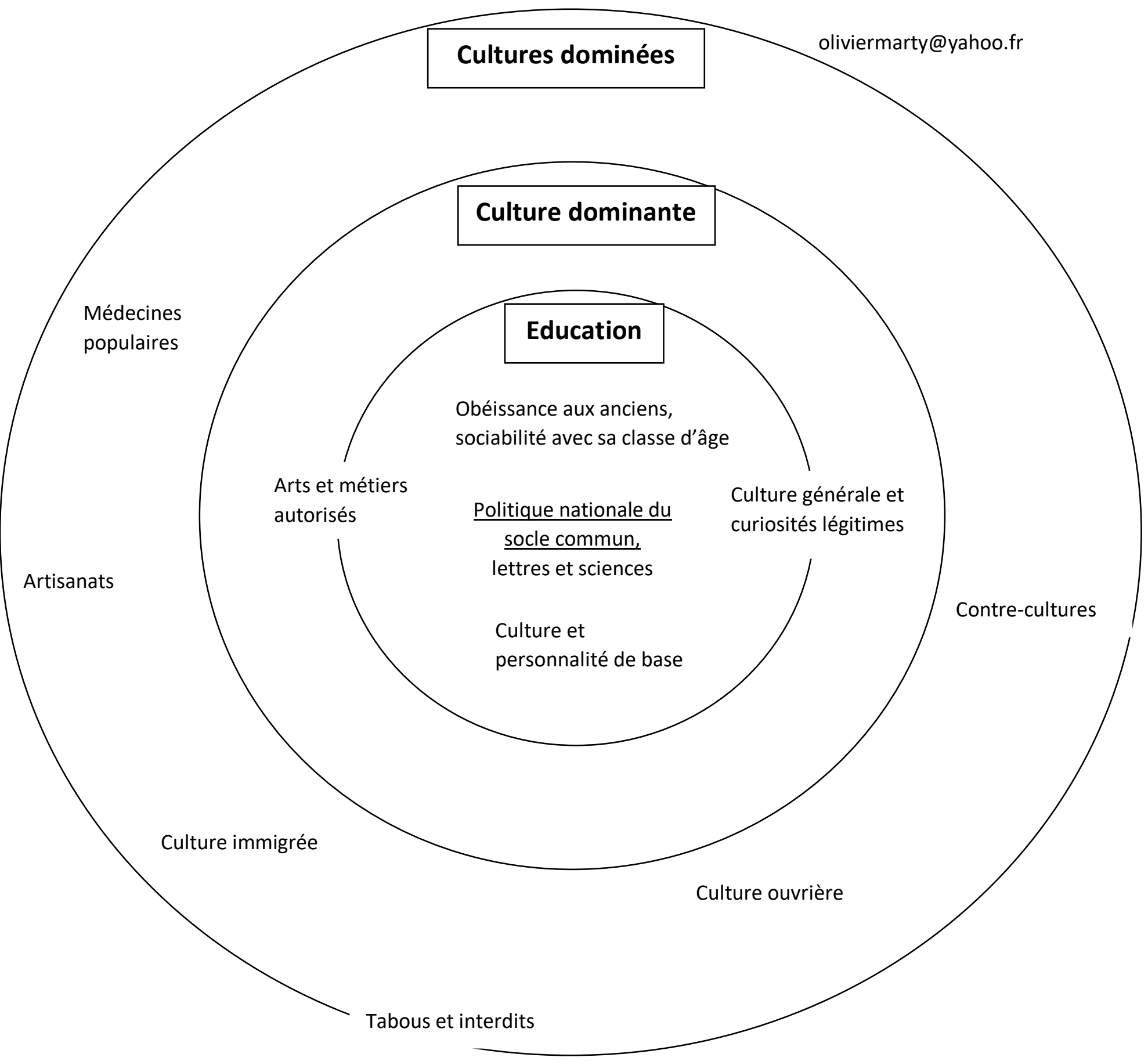

La transmission du noyau culturel par l'école : extensions 
L'évaluation par profession de ses pratiques se situe au cœur de ce modèle de transmission : un fils d'enseignant possède un art ou métier autorisé, une pratique familiale qu'il peut valoriser lors du concours de la Raep. L'autre partie de l'épreuve repose sur l'apprentissage scolaire du socle commun, voire de la culture générale - et la sociologie de l'éducation ${ }^{83}$ a montré comment ceci était fortement déterminé par l'origine sociale de l'apprenant.

Comment la distance modifie-t-elle ces enseignements ? L'image de la transmission du noyau culturel pour le faire germer dans l'esprit de la jeune génération peut être trompeuse : il ne s'agit pas d'un contenu figé à reproduire tel quel, des paroles mortes à ânonner. Bien au contraire, la formation telle qu'elle s'observe en actes consiste à apprendre à faire et à penser. Les compétences transmises sont des savoirs penser et des savoirs faire. Ce sont à la fois des techniques de corps au sens de l'ethnographe Marcel Mauss ${ }^{84}$ (domestiquer l'œil pour qu'il sache lire, la main pour qu'elle puisse écrire proprement, la bouche pour que ses muscles permettent de parler des langues étrangère) et des techniques de pensée telles que le conçoit le philosophe Michel Foucault ${ }^{85}$. C'est la mémoire, l'imagination, l'affection qu'il faut formater pour que les plus jeunes puissent penser, sentir et agir comme les anciens ${ }^{86}$.

Tous ces actes de formation sont dépendants du média par lequel ils sont exécutés. Apprendre l'anglais dans une classe de jeunes français, où il faut exercer son oreille à distinguer les sons émis par son professeurs de ceux, dissonants, de ses camarades, pour ensuite parler devant tous en maîtrisant sa pudeur ou sa peur du public, est très différent d'apprendre l'anglais avec une méthode individuelle en ligne : les sons présentés sont alors plus purs, on répète seul, sans retour sur la qualité de ce que l'on émet, sans personne pour railler ou féliciter, et à son rythme, au gré de ses envies. Cet exemple de l'anglais est valable

\footnotetext{
${ }^{83}$ CF. Les travaux de Pierre Bourdieu dans Les héritiers et La reproduction. Éditions de Minuit. France.

${ }^{84}$ Mauss M., 2002, Manuel d'ethnographie, Payot. La petite bibliothèque Payot. Paris.

${ }^{85}$ Foucault M., 1998, Surveiller et punir, Gallimard.Tel. Paris.

${ }^{86}$ La tentative d'emprise de l'école sur les esprits est totale, et ceci explique la proximité des pouvoirs religieux des œuvres éducatives: I'Eglise catholique et les jésuites en Occident, la madrasa musulmane.... Où même I'École républicaine française qui, en partie née de la Convention, apprend à lire et à écrire en français (et non en langues régionales) et à compter avec les poids et mesures établis par la Révolution française. Cette tentative n'est cependant pas toujours un succès comme le montre aujourd'hui l'échec scolaire et les autres instances socialisatrices (télévision, entreprise, armée...).
} 
pour tous les enseignements : on ne reproduit pas le noyau culturel de l'ancienne génération de la même manière selon que l'on apprend en présence d'un professeur ou à distance. ${ }^{87}$

L'expérience sociale de l'école est elle-même modifiée. La transmission du noyau culturel est souvent réservée à l'élite de la société : c'est la tradition du maître de philosophie chez qui le jeune noble de l'Antiquité est placé ou, plus humblement, celle du précepteur bourgeois au siècle des Lumières. L'éducation est alors un bienfait réservé à la classe aisée qui veut préserver ses enfants de l'oisiveté et de ses dangers. C'est aussi la réalité sociale de la reproduction des élites dans les grandes écoles françaises ou les universités prestigieuses anglo-saxonnes. C'est enfin et surtout la théorie de la classe de loisir du sociologue Veblen ${ }^{88}$, qui veut que seuls une élite oisive a le temps de se former, tout comme l'étymologie même du mot école qui renvoie au loisir et donc à la classe sociale qui peut se le permettre.

Au-delà du contenu de formation, des connaissances transmises, l'école est alors un lieu de reconnaissance où l'on apprend des codes communs qui permettront de se reconnaître dans la bonne société plus tard. Tous partageront les mêmes goûts, les mêmes attirances (ou valeurs), les mêmes savoir-faire (ou plutôt avoir su-faire) et les mêmes préjugés liés à leur rang : la formation est conformation, ciment de la nation, elle assure l'unité sociale - autour de la hiérarchie des positions héritées. Elle établit une hiérarchie entre ceux qui, sans éducation, devront innover pour conquérir leur place dans la société, ou reproduire une culture périphérique et populaire, et ceux qui pourront légitimement reproduire le meilleur de la société ancienne. L'école transmet des valeurs communes pour ceux qui seront au centre de la société, et, à son niveau le moins prestigieux, des valeurs spécialisées complémentaires qui préparent à des métiers déterminés.

Comme pour un rite de passage ${ }^{89} \mathrm{du}$ statut d'enfant commun à adulte supérieur, le processus social isole les jeunes prétendants (dans l'endroit de l'école, hors du monde du travail et coupé de la famille), leur fait passer une série d'épreuve (apprentissages vérifiés par des examens, tests) pour enfin leur donner le statut supérieur (via le diplôme). Que se passe-til dans le cas de l'enseignement à distance ? Ce rite de passage réservé à l'élite est modifié sans être supprimé ${ }^{90}$ : plus de groupe homogène, de promotion, plus d'épreuve commune clairement identifiée : tout se passe seul, isolé, loin de tous. Le fonctionnement même de

\footnotetext{
${ }^{87}$ Dans cet ouvrage, nous renvoyons au chapitre sur l'enseignement à distance à l'université de Corinne Baujard

${ }^{88}$ Veblen T., 1979, Théorie de la classe de loisir, Gallimard. Tel. Paris.

${ }^{89}$ Van Gennep A., 2011, Les rites de passage, A\&J Picard, Picard Histoire. France.

${ }^{90}$ Il reste parfois, par exemple, la cérémonie de remise de diplôme.
} 
l'institution scolaire comme rite de passage est peut-être à repenser : on touche là à l'éducation, c'est-à-dire à la relation sociale pure.

Pour conclure cette perspective critique, ce modèle d'évaluation normé, c'est-à-dire autorisé, n'est cependant pas si novateurqu'il peut paraître. En effet, l'épreuve de recrutement dans nombre de sociétés privées ou administrations publiques passe par le traditionnel curriculum vitae dont la rédaction - en adéquation avec l'offre d'emploi - est un art relativement comparable et tout aussi inégalitaire. Ici, l'évaluation est ramenée au récit biographique, toujours plus ou moins illusoire ${ }^{91}$, introduit et norme les jeux de présentation de soi $^{92}$.

\section{4/ Synthèse : conséquences sur la relation éducative apprenant-formateur}

\section{La relation éducative}

Nous avons défini l'enseignement par les signes à transmettre, la méthode aristotélicienne. À l'opposé, l'éducation est pédagogie, accompagnement de l'élève à la manière de l'Emile de Jean-Jacques Rousseau. C'est cette relation éducative, ce fait social, qui sera principalement transformé par la distance. Avant de détailler les modalités de la transformation, illustrons notre définition de l'éducation par un schéma qui nous servira de point de départ. Le schéma représente une relation inégalitaire entre un maître (point haut) et un élève (point bas), relation qui permet l'écoulement d'un courant d'idées, de signes ou d'enseignements. Cette relation est homophile, dans le sens où le maître et l'élève vont se rapprocher au fur et à mesure que l'éducation avance. La relation éducative a pour produit l'élévation sociale de l'élève.

\footnotetext{
${ }^{91}$ Bourdieu P., 1986, " L'illusion biographique ", Actes de la recherche en sciences sociales, $\mathrm{n}^{\circ} 62 / 63$.

92 Goffman E., 1990, The presentation of self in everyday life, Penguin.
} 


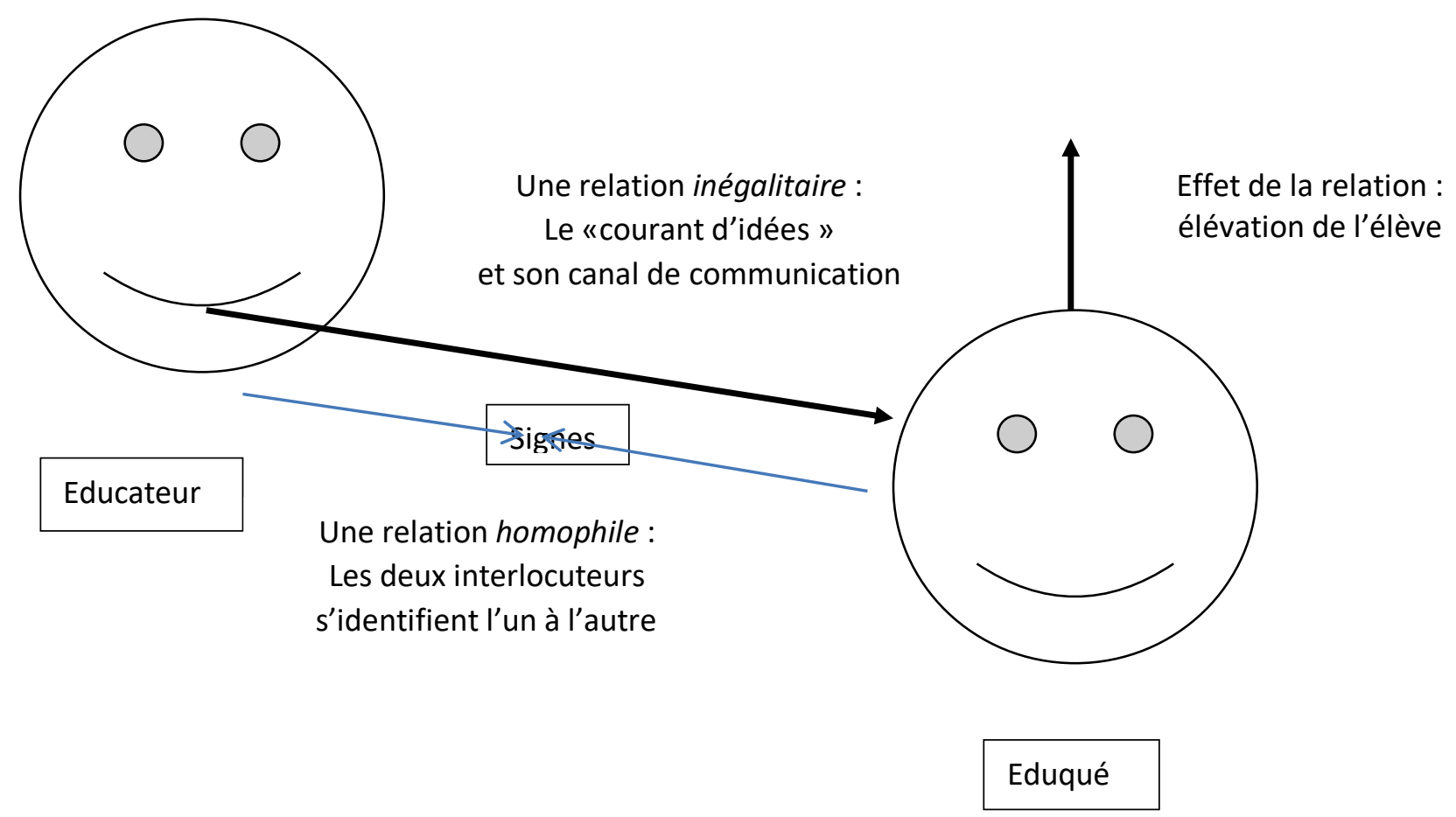

\section{La relation éducative}

Cet écoulement d'un point haut vers un point bas est vrai au niveau microsocial, entre un maître et un élève, mais aussi au niveau mésosocial entre des institutions d'enseignement hiérarchisées ou au niveau macrosocial entre des pays de pouvoirs culturels différenciés. Ainsi le Conservatoire national des arts et métiers rayonne et diffuse ses idées auprès d'universités créées dans d'anciennes colonies françaises, tout comme les idées et la culture américaines ont irrigué l'Europe de l'après seconde guerre mondiale. Toujours le courant d'idées va du point haut vers le point bas et les signes se diffusent en cascadant d'un pouvoir fort vers un pouvoir faible.

Voyons-en quoi l'insertion d'un média entre le maître et l'élève - papier, téléphone, télévision, Internet - ou ce que l'on appellera plus communément distance, va modifier la relation éducative. Nous confirmerons par là-même l'hypothèse que la relation enseignantenseigné est plus importante que l'enseignement qui transite, que la modalité technique du 
média, ou canal de communication, prime sur le type de signes transmis, pour comprendre les effets de la distance.

Nous présenterons d'abord ce qui est transformé dans la relation éducative par l'effet des médias, puis ce qui est conservé, notamment la métaphore théâtrale.

\section{Les effets des médias}

Les chercheurs anglo-américains ont étudié, média par média, l'impact de la technique sur la pédagogie. Ainsi plusieurs thèses ont été soutenues aux États-Unis ou au Canada sur l'utilisation du film, du téléphone ou encore de l'Internet pour se substituer au cours en salle de classe. Les dispositifs sociotechniques transforment la relation enseignant-enseigné ${ }^{93}$. Voyons ce qu'il en est, en France, dans le quotidien des agents publics du principal opérateur national. Détaillons les conséquences pédagogiques de chacun de ces média en les classant par ordre d'interactivité croissante.

Le cours sur support papier (de type livre ou fascicule) et le site Internet où ne peuvent qu'être téléchargés des documents (.pdf) sont les moins interactifs. Le centre d'enseignement met les cours à disposition de ses inscrits (étudiants collégiens, lycéens ou inscrits à l'université pour préparer, par exemple, un concours de la fonction publique) et ceux-ci peuvent faire un retour mensuel sous forme de devoirs que les enseignants corrigeront. Pour l'enseignant il n'y a aucun stress dans son travail puisqu'il n'a pas de dynamique de classe à gérer («faire la police», « réveiller la classe »). Tout au plus l'incertitude de savoir si son cours a bien été suivi et compris (l'enseignant ne peut pas lire dans les yeux des apprenants s'ils suivent bien comme il pourrait le faire dans une salle de classe). C'est ce métier traditionnel d'enseignant à distance, peu interactif et donc peu demandant émotionnellement, qui a conduit nombre d'académies à placer dans l'institution observée des personnels en maladie de longue durée. Les pathologies physiques (surdité partielle, aphonie...) ou mentales (troubles bipolaires) ne permettent plus d'assurer les cours dans la salle de classe mais s'adaptent bien aux contraintes allégées de l'enseignement à distance. Du côté des élèves, c'est une nouvelle façon d'apprendre à laquelle il faut s'adapter : avancer à son rythme et

\footnotetext{
${ }^{93}$ Voir par exemple la notion de distance transactionnelle chez Moore dans le Handbook of distance education opp. Cit. Ce même ouvrage liste dans ses premiers chapitres les thèses qui ont jalonné l'histoire des recherches nord-américaines sur l'enseignement à distance.
} 
librement suppose d'être capable de se fixer des contraintes, d'être autonome. L'apprenant doit être capable de diviser le travail en unités d'apprentissage régulières et de se «mettre au travail » tout seul, voire de surmonter une incompréhension ou une difficulté sans l'aide immédiate d'un enseignant. Aux élèves des DOM TOM ou des fils d'expatriés, public important de l'institution, s'ajoutent ainsi les professionnels voulant reprendre leurs études et ayant une plus grande maturité. Au final, la relation éducative distanciée par le cours papier est peu interactive, soulage le professeur et demande à l'apprenant de compenser par plus d'efforts personnels. Les efforts de l'institution, comme nous l'écrivions dans la première partie, visent à rétablir de l'interaction pour réintroduire plus de pédagogie. L'offre de formation, celle des enseignants rédacteurs de cours, est ainsi complétée par des services d'éducateurs : accompagnement, tutorat ou pédagogie par téléphonie ou sur forum.

Le téléphone rétablit la synchronicité dans la relation éducative. En cas de difficulté, l'apprenant peut appeler un professeur en charge de sa discipline pour obtenir une aide de méthode, ou même le responsable de formation pour obtenir des conseils sur l'organisation de son travail. Pour le personnel enseignant, la relation individuelle permet de prendre l'ascendant plus facilement que pour une question posée en public, dans la salle de classe. Le métier de l'enseignant à distance par téléphone est donc là aussi facilité. Le problème est que seuls les élèves les plus motivés appellent et ce sont donc les meilleurs qui voient leurs compétences renforcées.

Le forum sur site Internet permet de retrouver la dimension collective avec ses dynamiques tant positives que négatives. Les élèves peuvent poser des questions sur le forum de leur formation et un formateur veille à ce qu'une réponse soit fournie dans un délai de trois jours. L'enseignant à distance est relativement protégé par le caractère asynchrone de ses interventions (il n'est pas tenu de répondre immédiatement et ne sera donc pas « débordé » par le groupe) et par le fait que, invisible, il peut demander de l'aide à un collègue (il est donc rarement mis en porte à faux et tout élément perturbateur du forum peut être traité collectivement par le corps des enseignants). Les élèves ont souvent un bon sentiment à l'égard des forums, du moins les plus jeunes, car ils peuvent y chercher librement des informations parmi les questions qui ont déjà été posées. Ils permettent, qui plus est, de retrouver un esprit de classe que l'on n'avait pas dans les relations individualisées.

Le média qui produit le moins de distorsions par rapport à la présence est relativement récent : il s'agit de la classe virtuelle. Un enseignant peut, par le truchement d'un ordinateur 
connecté à Internet et équipé du logiciel approprié, animer un cours dans une classe virtuelle : il sera entendu et vu en direct par un groupe d'apprenants. Le son et l'image se combinent en temps réel, la situation se rapprochant de celle d'une salle de classe réelle. Le dispositif informatique demande cependant une certaine connaissance du logiciel puisqu'il faut pouvoir se concentrer sur l'enseignement tout en surveillant les différentes fonctionnalités éducatives : distribution de la parole aux élèves, retour par messagerie instantanée, partage d'application... afin de préserver l'attention des participants, il est conseillé aux enseignants de souvent poser des questions. Ils procèdent alors sous forme de sondages et s'assurent ainsi que tous les étudiants suivent.

$\mathrm{Au}$ final, malgré les avancées techniques importantes des vingt dernières années (forum du site Internet, classe virtuelle), l'enseignement à distance entraîne un affaiblissement de la relation éducative. Les canaux de communication sont plus fins et seuls l'écrit, la voix (téléphone) et la vision (classe virtuelle, film) sont transmis : pas de relation touchante, pas de ressenti lorsque les élèves apprennent à distance. La relation éducative n'est plus multisensorielle. À cela il faut ajouter une interactivité moindre (surtout dans le cas du papier) et, lorsqu'il y a interactivité, elle n'est pas toujours synchrone. Nombre de média décalent la question et la réponse dans le temps, introduisant une distance temporelle dans la relation éducative.

Une note interne de l'institution, émanant, en janvier 2012, de la direction de l'innovation, classait les différents médias par le degré d'ambigüité. Ainsi les médias les plus interactifs seraient les plus ambigus : cette vision conservatrice place le papier ou le fichier à télécharger comme peu ambigu (un seul message clair est transmis) alors que le téléphone ou la classe virtuelle seraient plus ambigus (car le ton de la voix peut contredire la parole, les mouvements du visage contredire les mots). Nous allons visualiser cette théorie à l'aide d'une carte mentale. Là où un enseignement en salle de classe est parasité par la richesse des canaux de communication et les circonstances d'énonciation (la déictique produit parfois des injonctions paradoxales), un message transmis par papier est plus clair car plus pauvre. 


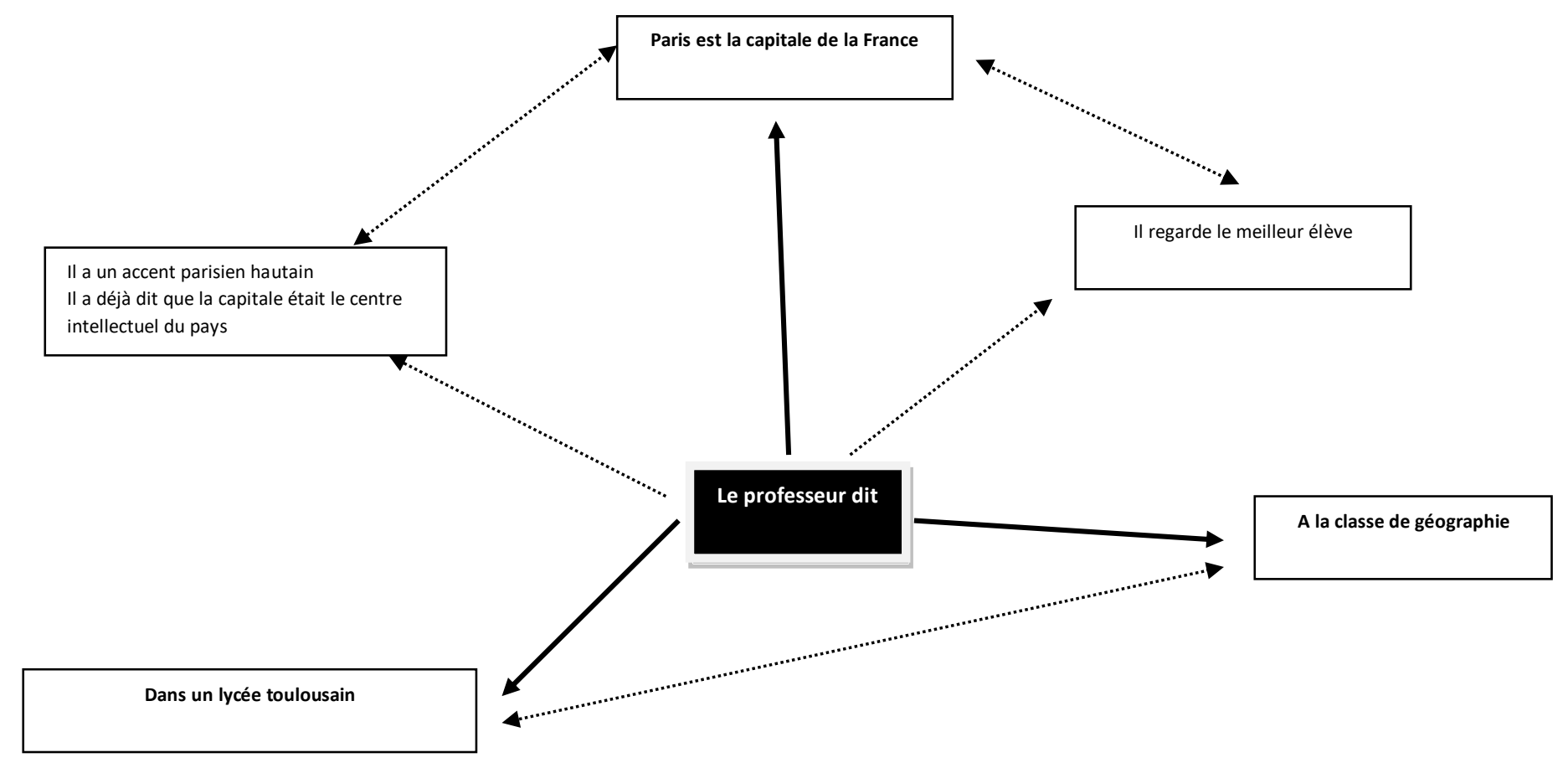

Carte des échos de signification : cours de géographie

Lorsque le professeur dit «Paris est la capitale de la France» à la classe de géographie, dans un lycée toulousain, après avoir expliqué que Paris était le centre intellectuel du pays, ce message clair peut être parasité par des informations secondaires. Ainsi, le fait qu'il regarde le meilleur élève au moment où il dit ceci et le fait qu'il a un accent parisien hautain donnent au même moment des informations sur 1/ son positionnement hautain par rapport à la salle de classe (il se dit parisien par son accent et s'adresse à des toulousains, ville qui n'est pas la capitale) et $2 /$ son positionnement encourageant par rapport au meilleur élève (il le regarde comme si Paris lui était associé puisque c'est le centre intellectuel).

Le même message « Paris est la capitale de la France » écrit dans un cours sur support papier et envoyé également, sans accent ou regard, à tous les inscrits, sera moins ambigu. Aucun écho de signification ne viendra parasiter le message. L'écrit est plus neutre car il 
épure l'enseignement. Le média a ainsi un effet réducteur qui diminue les ambigüités entre l'émetteur et le récepteur.

Le métier d'éducateur est donc modifié par la distance : plus sécurisé, moins stressant, car moins interactif ; la relation d'enseignement est plus efficace sur certains points car plus épurée quant aux messages transmis. Voyons, à l'inverse, une vision du métier qui est stable et qui se conserve lorsque l'on passe de la présence à la distance.

\section{La conservation de la métaphore théâtrale}

Comme nous l'avons montré dans le dossier ${ }^{94}$ que nous avons dirigé dans la revue Education Permanente sur le thème «La formation par le théâtre », les liens entre le théâtre et l'éducation sont multiples. L'enseignement peut alors être vu comme un art plus que comme un métier.

Dans les Politiques, Aristote fait du spectacle un outil d'éducation. Thème qui sera repris au siècle des Lumières dans une controverse philosophique célèbre portant sur la création d'un théâtre à Genève : les textes mis en scène sont-ils œuvre d'éducation populaire ou au contraire décadence et perversion des mœurs ? Car il est sous-entendu que l'on apprend (ou désapprend) lorsque l'on est placé comme spectateur.

Aujourd'hui, c'est en étant acteur que l'on apprend : les élèves sont mis sur le devant de la scène par un professeur metteur en scène, pour improviser en équipe, pour apprendre à parler en public, pour maîtriser leurs émotions.... voire pour purger, par catharsis, des traumatismes.

Le lien entre théâtre et formation qui retient notre attention est le plus évident : c'est celui qui fait du théâtre une métaphore pour comprendre l'enseignement en salle de classe. Plusieurs livres de didactique font en effet l'analogie entre le professeur-acteur avec son rôlecours présenté sur l'estrade-scène devant des élèves-spectateurs attentifs. L'acte d'enseignement est un véritable spectacle, parfois costumé (en blouse blanche de physicien), souvent simplement déclamatoire car les actes joués en interaction avec l'audience (le jeu de questions-réponses, l'adaptation au niveau des élèves) sont les plus difficiles. Est-ce que la relation éducative, vue comme relation théâtrale, est modifiée par la distance ?

\footnotetext{
${ }^{94}$ Marty O. (dir.), 2013, dossier : " Théâtre et formation », Education Permanente, opp. Cit.
} 
Alors que l'on pourrait croire que la distance supprime le spectacle, il apparaît, au sein de l'institution étudiée, que les termes mêmes de cette métaphore sont repris pour comprendre les pratiques professionnelles innovantes. Ainsi, le travail de formatage des enseignements pour les rendre compatibles avec une interface informatique est appelé, au sein de l'institution nationale : «scénarisation ». Il s'agit de mettre en scène le contenu de formation, de le présenter à l'internaute de façon à ce qu'il puisse naviguer aisément sur un poste informatique. L'enseignant n'est plus directement sur le devant de la scène : il tire les ficelles d'une machine qui accomplit l'acte éducatif à sa place, en relation directe avec les apprenants.

Au-delà du vocabulaire vernaculaire, nous soutenons que la métaphore théâtrale est plus profondément encore conservée par les gens du métier lorsqu'ils passent à la distance. En effet, le theatron, en grec ancien, est le lieu où l'on voit. Et cette essence visuelle de l'acte d'enseigner est conservée dans l'établissement public administratif par les nombreux dispositifs audiovisuels dans lesquels l'institution investit. Les studios d'enregistrement de l'établissement public diffusent en effet des vidéos, soit sous forme de films (documentaires, spots publicitaires), soit à destination des sites Internet (courtes vidéos, jeux vidéo). Ainsi, il s'agit encore de visualiser ce qui est enseigné, de théâtraliser l'enseignement.

C'est une de forces principales de cette industrie de la connaissance : sa division du travail est suffisamment poussée pour disposer de moyens d'investissement importants et concentrés sur des activités visuelles constituant le cœur du métier.

\section{$\underline{\text { Conclusion }}$}

Notre terrain dans l'enseignement à distance, parmi les ingénieurs de formation concevant les dispositifs de préparation à l'épreuve de Raep des Capes et agrégation, nous permet de comprendre les conséquences de cette norme d'évaluation ${ }^{95}$ consistant en une profession de ses pratiques :

- au niveau des sciences de l'apprendre, du point de vue de l'apprenant qui doit développer des qualités nouvelles: réflexion personnelle, pratiques variées, formalisation selon des référentiels, verbalisation intelligente

\footnotetext{
${ }^{95}$ Le dossier numérique de compétences, exemple d'e-portfolio
} 
- au niveau de la didactique, du point de vue du formateur qui fait primer l'accompagnement pédagogique sur l'enseignement de contenus et voit ainsi son métier évoluer - et ce bien que des inégalités macro-éducatives critiquables persistent dans la transmission du noyau culturel.

- enfin au niveau synthétique de la relation éducative entre apprenant et formateur où, dans le cas de l'enseignement à distance, nous assistons à un épurement de l'enseignement sous l'effet de la médiatisation des contenus, tout en conservant une dimension visuelle (ou théâtrale) importante

Ainsi un nouveau type d'évaluation, consistant à professer ses pratiques, normalise les pratiques éducatives selon les nouvelles voies que nous avons tenté de montrer. Notre chapitre à visée heuristique pourrait alors être résumé par l'idée de nouvelle standardisation des pratiques. Il laissera à présent sa place à une observation propre à notre position d'observateur, voyant, en matière d'évaluation, l'économique contraindre le docimologique. 
Nos données de terrain sont une observation participante dans une réunion où sont fixés les tarifs de rémunération de correcteurs des différentes préparations nationales aux concours d'agrégation du secondaire (mathématiques, physique, sciences économiques et sociales, philosophie, espagnol, anglais, etc.). Nous inscrivons ces données empiriques dans le cadre théorique de la littérature sur la comparabilité : deux ouvrages français vont nourrir notre réflexion : la Sociologie économique de la mesure de François Vatin (2013) qui pose la question de la mesure de la valeur du travail de formation et La politique des grands nombres d'Alain Desrosières (2010) qui introduit à une réflexion sur la comparaison et la comparabilité des notes des apprenants pour renouveler le débat sur la quantité et la qualité. La réception française de la théorie de la valuation de John Dewey par Alexandra Bidet (2011), Louis Quéré et Gérôme Truc est aussi signalée car elle permet de comprendre comment sont valorisés les actes évaluatifs. Enfin la Grammaire des conduites à projet de Jean-Pierre Boutinet (2010, p.196) offre une perspective sur l'acte d'évaluation comme création de valeur dans une industrie de la connaissance telle que la décrit Pierre Moeglin (2012). Nous nous situons non pas du côté de l'élève (voire de la communauté éducative) et de sa lecture des annotations, non pas du côté de l'enseignant signataire de l'annotation, mais de celui du cadre intermédiaire de l'enseignement qui doit mesurer la valeur d'une notation, pour le concours d'agrégation du secondaire.

Suivant cette méthode et consultant la littérature précitée, notre question de recherche est : quel est le lien entre la valeur économique d'une notation (le tarif de rémunération d'un correcteur) et sa valeur docimologique (la production de sens d'une note chiffrée et d'une annotation par le correcteur dans sa discipline) ? Nous questionnerons ce lien entre les mesures de valeur au travers de notre description de terrain pour ensuite ébaucher une théorie plus large de la démesure en matière d'évaluation - offrant ainsi une perspective macrosociale sur le débat quantité/qualité en matière d'évaluation.

\section{$\underline{\text { Observations de terrain : fixer la valeur des évaluations }}$}

L'administration observée est en charge du service public de l'enseignement à distance. Instituée en 1939 et comptant huit sites d'exploitation sur le territoire national, Elle emploie des « responsables de formation chargés d'ingénierie de formation » qui encadrent 
des enseignants à distance : auteurs, correcteurs, tuteurs,... collaborant sur des dispositifs papier ou électronique. Les correcteurs sont ainsi en charge de copies papier et électroniques d'apprenants pour lesquels ils n'assurent ni le tutorat (téléphone, échange de méls,...), ni la délivrance des enseignements premiers (livres, fascicules sur environnement numérique de travail, etc.). Ces correcteurs sont recrutés dans le corps de professeurs agrégés ou certifiés de l'éducation nationale, ils peuvent aussi être vacataires cherchant un complément de rémunération. L'art de la notation qu'ils exercent est relativement libre : chacun relevant d'une discipline qu'il a apprise puis pratiquée en milieu scolaire ou universitaire, ils peuvent évaluer les copies selon leurs connaissances et habitudes. La notation fait le plus souvent l'objet d'un barème dont la somme est de vingt points - selon la tradition nationale, les annotations explicatives sont encouragées pour aider les apprenants à combler leurs lacunes et développer leurs points forts. Cet art est cependant cadré par un métier : les outils de la communauté professionnelle appartiennent à l'administration étudiée ; ce sont le matériel de correction (bureau, ordinateur, connexion Internet, etc.) mais aussi et surtout la rémunération des vacataires.

Les responsables de formation ont la charge de coordonner l'organisation ou appareil administratif de chaque formation à distance - ici telle ou telle préparation à l'agrégation - et en particulier la rémunération des correcteurs. Ceci fait l'objet de nombreux tableaux de déclaration informatisés des personnels employés dans lesquels sont indiqués les tarifs de correction. La réunion que nous allons décrire est le moment où les quinze responsables de formation du site sont réunis par la chef de service pour arrêter une nouvelle tarification. Les nécessités de coordination entre sites d'exploitation - l'institution en comptant huit au total demandant de réduire les dépenses de paiement des vacataires et de faciliter leur gestion en harmonisant les taux de paiement.

$\mathrm{Au}$ cours de la réunion, nous avons pu ouvertement observer les différents débats et arguments mobilisés. Le discours gestionnaire central était de minimiser le nombre de tarifs en vigueur. La logique était un taux horaire, débouchant sur trois tarifs : différents selon le niveau (agrégation versus Capes) mais communs quelle que soient les disciplines. La logique du taux horaire devait permettre d'harmoniser la correction des copies de mathématiques avec celle de lettres, d'anglais ou d'histoire. Elle différenciait uniquement les copies de niveau agrégation - censées demander plus de temps - des copies de Capes. Une exception a été débattue pour le cas d'épreuves spécifiques imposant un temps de correction plus long. Ainsi une épreuve de lettres classiques a vu son tarif maintenu de trente pourcent au-dessus du tarif des autres disciplines du fait de sa complexité. 
La logique du temps de correction a aussi prévalu lors du débat autour des copies orales. Le dispositif de transfert électronique des copies permet en effet de recevoir des fichiers audio dans lequel les préparationnaires ont enregistré leur prestation orale. La question a alors été de savoir s'il fallait rémunérer à un tarif supérieur ces copies à celui des copies écrites, plus rapides à corriger. Une décision collective a abouti à un tarif légèrement différencié. En effet, il a été avancé que le correcteur devait écouter l'enregistrement et que cette opération lui demandait plus de temps que de lire une copie écrite - sous format papier ou électronique. La même logique du temps de correction a donc prévalu pour déterminer le tarif de rémunération.

Notre observation participante nous permettant d'assister aux discussions informelles dans les couloirs et à la pause-déjeuner, nous pouvons rapporter que d'autres critères de paiement - non officialisés par la réunion de service et ses débats - avaient prévalu par le passé : prime à l'utilisation d'une nouvelle technologie (pour convaincre les correcteurs indépendants de corriger par voie électronique et non papier, un supplément leur avait été concédé), prime à la qualité du correcteur et surtout accumulation de strates tarifaires différenciées au cours de l'histoire de l'institution, conservant des correcteurs rémunérés avec des tarifs de leur premier contrat - dans le même site d'exploitation il y a quelques années ou venant d'autres sites d'exploitation avec des tarifs différents.

Le débat de la réunion a toutefois été simplifié autour du taux horaire et de la discussion sur le temps pour corriger tel ou tel type de devoir. Cette économie de la durée se fonde sur une évaluation du tarif de correction d'une copie qui dépend du temps estimé, luimême dépendant soit du niveau (agrégation/capes) soit du support (audio/oral), soit d'une particularité irréductible (épreuve de lettres complexe). Comment analyser les conséquences docimologiques de cet acte administratif d'économie de l'évaluation?

Analyse : une docimologie microéconomique

Nous posons que, dans le cas de l'administration observée, la relation éducative entre correcteur et corrigé n'a pas le temps de s'établir au fil d'une année (voire plus) de formation. Nous constatons une méconnaissance des auteurs des copies par les correcteurs, de l'impossibilité de déterminer les progressions ou attentes particulières. Dans ce type d'industrie de la connaissance, il s'agit d'optimiser la production et la rémunération « à la pièce » de la copie corrigée à des conséquences sur la docimologie. 
En effet, la motivation du correcteur est étroitement dépendante, non plus des progrès de l'élève ou même de l'adéquation avec le cours - puisque, rappelons-le, ce n'est pas le correcteur qui le délivre - mais de la rémunération à la copie. L'art de la correction est formaté par le métier administratif ; et le temps prévu, traduit en rémunération, influe sur la correction par le biais de la motivation. Les disciplines universitaires et leurs différences de méthodes, d'approche, de sensibilité, sont formatées dans un même cadre organisationnel qui s'impose à tous. Une métronomie commune du travail de correction et de sa valeur s'impose, l'évaluation économique originelle de l'acte docimologique d'évaluer une copie vient cadrer l'art des correcteurs. Noter à l'économie est ainsi une conséquence première et évidente: selon le taux de rémunération, un temps est passé et un effort est fourni sont accordés.

L'art de la pratique de notation sont cadrés par le métier ou l'organisation de l'appareil administratif rémunérateur. L'économique prime sur le docimologique. L'évaluation première, celle des correcteurs et de leur travail de correction, est économique. Elle se mesure en temps linéaire - de travail - qui ne tient pas compte des variations de densité. Cette métronomie temporelle ne va pas sans rappeler le formatage européen du système éducatif supérieur par l'European Credit Transfert System : là-aussi, la mesure des formations est normée par le temps moyen supposé nécessaire pour acquérir les apprentissages - validés par l'examen.

Cette économie de la quantité de temps est certes une simplification utile, elle permet cependant difficilement d'évaluer la haute qualité. Essayons à présent de montrer comment les niveaux supérieurs (en particulier au-delà du master, par exemple le doctorat), ne rentrent pas dans ce système de mesure chiffrée et reposent sur un moment fort, hors du temps linéaire quantifiable.

\section{L'élitisme et la démesure en évaluation : le temps qualitatif}

Lorsque nous comparons notre observation-participante de type auto-ethnographique à une expérience d'apprentissage personnelle - notre cérémonie de soutenance de thèse de doctorat universitaire en philosophie, voire les jurys de soutenance que nous avons présidés pour des mémoires de masters en grande école, ou encore la participation à un jury de thèse en sciences de l'éducation - nous nous apercevons qu'une logique différente prévaut.

En effet, au-lieu d'une évaluation standard de la correction de copies anonymes d'agrégation, selon des corrigés-types et un taux-horaire impliquant une notation à l'économie, nous assistons dans ce deuxième cas à un suivi individualisé tout au long de la 
formation et de la production du document à évaluer : orientation, suivi, aide, du directeur de thèse ou du directeur de mémoire jusqu'à la reconnaissance finale collective lors de la cérémonie de soutenance (organisée par le même directeur). Le temps de correction s'étire ainsi dans une année de master ou trois années de thèse - selon les normes européennes de mesure de la valeur d'une formation précitées - mais se condense dans un moment hautement qualitatif qui est celui de la cérémonie collective de soutenance.

Cette cérémonie ne fait pas l'objet d'une note chiffrée : les annotations correctives tout au long de l'année ont permis d'arrêter le document final qui est déposé face au jury et à destination de l'université, idéalement soumis, parfois défendu. Une cérémonie stable permet la discussion libre autour du document, une soutenance innovante s'appuie sur une lecture prudente du document et une réponse formelle aux questions des interrogateurs. Le diplôme qui en dépend - master ou doctorat - fait mention d'une annotation précisant sa qualité. Celles-ci, sans être totalement libres, ne sont pas chiffrées, ni même normées dans une grille standard. La référence à la valeur de «l'honneur», plus ou moins grand, voire de la « félicité », accordée plus ou moins unanimement, traduit une qualité concédée à l'apprenant.

Le directeur de master et le directeur de thèse de doctorat sont rémunérés pour leur travail après la cérémonie de soutenance - selon une moyenne horaire fixée par l'organisation administrative qui délivre le diplôme. C'est donc ce moment fort et qualitatif qui condense la ou les années de corrections actives. Il est lui-même de durée variable, d'une heure à une demi-journée, hors du temps linéaire.

Notre interrogation finale, concernant les usages sociaux de la statistique, est celle la comparaison qu'autorise le chiffre. En effet, alors que, dans le cas de la préparation à l'agrégation, l'économie des métiers fixant la rémunération des correcteurs a des conséquences sur l'art docimologique de leur notation (évaluation chiffrée dans les deux cas : taux horaire et note sur vingt), dans le cas de la cérémonie de soutenance de thèse, le moment intense échappe à la mesure linéaire du temps (évaluation qualitative dans les deux cas : présence d'experts éminents et mention sur le diplôme). Alors que la masse des étudiants scolaires et universitaires suivent un corps professoral agrégé et font l'objet d'une correction normée, quantifiée et linéaire (la note qui permet de comparer et classer les copies), l'élite constituée par les doctorants entre dans une économie de la démesure où la valeur de leur diplôme échappe au chiffre et donc à la comparaison. La langue de travail, la réputation de l'université, la discipline, la composition du jury, la mention du diplôme sont difficilement quantifiables et résumables en un chiffre pour faciliter la comparaison. La notion même de promotion au sein de laquelle s'insérer pour une mise en équivalence dans une économie de la 
grandeur cède sa place à un sujet de thèse qui fait l'actualité de la recherche lors de sa conception. Noter à l'économie devient alors impossible et l'art du correcteur est d'économiser ses notes en les faisant partager par un jury évaluateur. C'est l'acte même d'évaluer qui prend de la valeur lorsqu'il est supporté par un collectif de qualité, transférant son degré de vérité au document du docteur conduit, éduqué puis corrigé.

\section{Conclusion : de l'anthropologie économique à l'anthropologie épistémique}

Notre itinéraire d'observateur participant et de participant observateur dans l'enseignement à distance, et en particulier le chapitre précédent sur le lien entre docimologie et économie, pourrait laisser penser que notre anthropologie se réduit à une anthropologie économique critiquant un gouvernement par les nombres surrané. Nous avons en effet, selon une approche institutionnaliste ou organisationnelle classique en anthropologie de l'éducation, étudié une industrie éducative diffusant les connaissances. Notre originalité étant d'étudier non pas comment les connaissances sont produites (par la recherche) ou consommées (par les étudiants) mais l'intermédiaire éducatif qui permet de les diffuser (l'industrie commerciale d'un centre d'enseignement à distance, appareil administratif national). On retrouve bien des problématiques propres à l'anthropologie économique qui occupe une place de choix dans les sociétés de la connaissance - où la connaissance se massifie, se marchandise et devient un secteur d'activité parfois lucratif. Est-elle un bien public ou un bien privé ? Cette distinction révolutionnaire et pourtant acutelle avait-elle un sens dans l'université médiévale ?

Pourtant nous ne voulons pas réduire nos efforts à une anthropologie économique. On remarquera en effet l'évolution dans notre ouvrage depuis la gestion, vers la conception et enfin l'apprentissage. La description du métier de cadre intermédiaire de l'enseignement à distance, spécialisé dans les fonctions d'ingénierie, laisse peu à peu la place à des descriptions sur le métier d'élève ou encore sur les activités d'apprentissage et leurs valeurs.

Nous pensons que notre anthropologie économique n'est pas insensible au type de produit ou service éducatif qu'elle étudie. Analyser la diffusion des connaissances ouvre la voie à une perspective d'anthropologie épistémique sur le rapport de l'homme aux savoirs, rapport à la fois sociétal, organisationnel et psychique selon le degré de précision de l'observation. Une anthropologie des métiers de l'enseignement à distance peut s'ouvrir sur des questions telles que : quelle est la place des connaissances (publiques ? privées ?) 
diffusées dans la société en son ensemble ? Quelles sont les valeurs et transformations opérées par les organisations qui diffusent ces connaissances ? Quel est le mode de réception et le rapport au savoir individuel des étudiants de cette institution?

Nous pensons ainsi contribuer par notre anthropologie épistémique aux sciences studies. D'une part car la connaissance n'est pas insensible à son économie et parce que son mode de diffusion détermine sa nature. Là où les sciences studies s'intéressent d'abord à la production des connaisances par la recherche, nous voulons compléter cet effort par notre programme d'investigation sur la diffusion de ces connaissances par l'appareil éducatif. D'autre part, et enfin, car notre épistémologie propre à ce travail appelle une réflexion relevant de l'anthropologie épistémique.

Nous allons donc présenter dans ce chapitre conclusif une revue de littérature précisant l'anthropologie de l'éducation annoncée en introduction par l'anthropologie des savoirs telle qu'elle se partique aujourd'hui. Puis nous montrerons les différentes anthropologies que nous avons mobilisées pour réaliser notre étude. Enfin nous achèverons l'ouvrage par des considérations épistémologiques réfléchissant à notre propre travail, à la fois d'anthropologue et de praxéologue.

\section{Revue de littérature : préciser l'anthropologie de l'éducation par celle des savoirs}

La revue de littérature montre l'actualité de l'anthropologie du fait éducatif, au travers de diverses terminologies évolutives vers la notion de savoir. Le sociologue Edgard Morin appelait, en France et dès les années 1970, une anthropologie de la connaissance, dans son troisième ouvrage grand public sur la Méthode ${ }^{96}$. C'était dans l'optique de mieux connaître nos connaissances (la « connaissance de la connaissance » selon l'approche «méta » qui le caractérise) et ceci préfigurait donc l'anthropologie des savoirs. Son spectre était large puisqu'il avait recourt à des connaissances biologiques (notamment neurologiques) et sociales pour d'écrire l'homme dans l'action de connaissance.

Le philosophe historien des idées Michel Foucault prônait, au même moment, lui aussi une archéologie du savoir incluant cette dimension anthropologique comme il allait le préciser dans ses Dits et Ecrits ${ }^{97}$ de 1976. Il s'agissait alors de se positionner en ethnographe de notre propre culture et de ses schémas de pensée pour la traiter comme étrangère et en voir de ce

\footnotetext{
${ }^{96}$ C'est le titre-même du chapitre majeur qui début dès la page 33 dans Morin E., 1986, La méthode. 3 : la connaissance de la connaissance, Seuils. Points Essais. France.

${ }^{97}$ Foucault M., 2001, Dits et écrits, tome 2 p. 160-174, leçon du 7 janvier 1976, p. 164. Gallimard. France.
} 
fait les grandes lignes architecturales apparaître. Cette perspective ne pouvait que passer par des études historiques dont il s'est montré une figure exemplaire et qui reste encore d'actualité dans l'anthropologie des savoirs ${ }^{98}$.

En parallèle, en Allemagne, Wulf s'établissait dès les années 1980 comme un spécialiste international de l'anthropologie de l'éducation, bien que peu de ses ouvrages aient été traduits dans notre langue. Dans son livre Anthropologie de l'éducation ${ }^{99}$, il aborde lui aussi les questions de connaissance et de «l'anthropologie pédagogique » autour de l'apprentissage, la malléabilité ou «la perfectibilité » de l'homme, ce qui ne va pas sans rappeler plusieurs traditions philosophiques sur l'éducabilité (Rousseau en France et Nietzche en Allemagne, dans les pays que nous évoquons).

Cette anthropologie de l'éducation va dans le sens des recherches nord-américaines dans ce champ ainsi désigné et ayant fait l'objet de manuels ${ }^{100}$. Les auteurs du nouveau monde accordent une place importante à l'influence des analyses bourdieusiennes et de la french theory. Ainsi la noblesse d'État, la reproduction ou les héritiers sont mis en perspectives avec les réalités états-uniennes pour critiquer des institutions où le pouvoir est blanc ${ }^{101}$ et masculin ${ }^{102}$. Les analyses sur le système français de l'état providence et sa stratification originale sont adaptées pour une dénonciation du capitalisme universitaire et des politiques éducatives néolibérales qui marchandisent l'enseignement ${ }^{103}$, dénonciations qui trouveront écho en France comme par exemple dans le colloque de Nantes de 2013. Nous pensons que la question de la monétisation de l'éducation est à replacer dans des cultures nationales et doit prendre garde à comptabiliser les coûts complets d'un acte éducatif (logement, coût d'opportunité du temps passé, etc.) avant de le critiquer. Ceci rejoint la

\footnotetext{
98 Par exemple : Jacob C., 2011, Pour une anthropologie historique des savoirs. Conférence présentée le 4 février 2011 devant la Société française pour l'Histoire des Sciences de l'Homme, Paris.

${ }^{99}$ Wulf C., 1999, Anthropologie de l'éducation. L'Harmattan. Savoir et formation. France.

${ }^{100}$ On peut faire reference au manuel : Dearborn G., Louise S., Fifty Years of Anthropology and Education, 1950-2000 : A Spindler Anthology, Publisher Lawrence Erlbaum Associates.

${ }^{101}$ Brayboy, Bryan McKinley Jones, 2003, The Implementation of Diversity in Predominantly White Colleges and Universities. Journal of Black Studies, Special Issue 34(1):72-86.

102 Holland, Dorothy C., and Margaret A. Eisenhart, 1990 Educated in Romance: Women, Achievement, and College Culture. Chicago, IL: University of Chicago Press.

Mir, Shabana, 2009, “Not too 'College-Like,' Not too Normal: American Muslim Undergraduate Women's Gendered Discourses." Anthropology \& Education Quarterly, 40(3), p. 237-256.

103 Barrow, Clyde W., 1990 Universities and the Capitalist State: Corporate Liberalism and the Reconstruction of American Higher Education, 1894-1928. Madison, WI: University of Wisconsin Press.

Canaan, Joyce E., and Wesley Shumar, 2008 Structure and Agency in the Neoliberal University. New York: Routledge.

Shumar, Wesley, 1997 College For Sale: A Critique of the Commodification of Higher Education. Washington, DC: Falmer Press.
} 
question plus large de savoir si l'éducation est un bien public ou un bien privé (ce qui détermine en partie sa monétisation).

La littérature anglophone fait aussi état de l'anthropologie de la connaissance («knowledge ») elle aussi depuis les années 1980, avec des auteurs comme Barth, Elkana ${ }^{104}$, Cohen ${ }^{105}$ ou Crick, menant chacun des recherches indépendantes sur ces thématiques dans différents continents. Crick a tenté une synthèse précoce des apports sur ces sujets. De ces recherches anglophones, qui ont l'avantage d'être portées par une langue d'usage importante dans les sciences contemporaines occidentales, il ressort que le terme de «savoir » (ou, pris sans nuance, « connaissance ») est préférable à l'approche traditionnelle d'une anthropologie de l'éducation telle qu'elle continue à persister en Allemagne ou sous les formes dont nous avons montré les limites aux États-Unis. Nous précisons donc notre anthropologie de l'éducation en une anthropologie des savoirs en référence à cette tendance des auteurs de langue anglaise.

Il reste de ces mouvements scientifiques trentenaires aujourd'hui en France une revue et une société d'anthropologie de la connaissance, et la thématique est, en 2017, listée dans la section 38 du CNRS (anthropologie) sous la désignation «anthropologie des savoirs, de la connaissance et du symbolique » (la notion d'anthropologie de l'éducation ou pédagogique en est absente). Cette thématique de recherche est ainsi centrale de l'anthropologie scientifique française.

Ceci peut se confirmer quand on regarde les thèses de doctorat des universités soutenues en France sur le sujet, pour n'en citer que trois :

- Duvillard J., 2014, Lyon, «La place des gestes et micro-gestes professionnels dans la formation initiale et continue des métiers de l'enseignement ». On y voit la dimension professionnelle et donc la question qui nous est chère des métiers apparaître.

- Dutournier G., 2014, Inalco Paris, «Description d'une relation lettrée dans la Chine des Song : un essai d'anthropologie du savoir. Cette thèse est de nature plus historique et correspond à un courant de l'anthropologie des savoirs laissant une place majeure au temps long.

\footnotetext{
${ }^{104}$ Elkana, 1981, “A programmatic attempt at an anthropology of knowledge” Sciences and cultures, Springer ${ }^{105}$ Cohen E., 2010, "Anthropology of Knowledge" Journal of the Royal Anthropological Institute.
} 
- Wathelet O., 2009, «Anthropologie de la transmission des savoirs et savoir-faire sensoriels ». Cette anthropologie-là a le mérite de questionner la sensualité de la connaissance (ici par l'osmose), ce qui va dans la même direction que nos travaux de sémantique sur la notion de savoir et le champ lexical de la saveur.

Le dynamisme du champ est porté en France par Béguin et Verbuge rappelant la proximité entre les problématiques d'éducation et celles de communication en matière de savoirs. La thématique étant par ailleurs proche de nos travaux puisqu'elle accorde une large place à l'entrée « activité », par une anthropologie des savoirs en action.

Le résumé français que nous privilégions reste cependant le manuel de 2011 de Nicolas Adell (Anthropologie des savoirs) ${ }^{106}$, quoiqu'il opère des choix dans les auteurs (Lévi-Strauss de la Pensée Sauvage, Descola de Par-delà Nature et Culture, par exemple) et concepts présentés (magie, initiation...) qui le rapprochent plus de l'anthropologie traditionnelle par aires culturelles que des sciences de l'éducation et des objets tels que ceux que nous avons travaillés (organisation, conception, apprentissage,...). Un chapitre correspondant à nos travaux porte sur la notion d'identité et apporte un regard radicalement différent du nôtre, centré sur les notions de transformation plus proche de Mokhtar Kaddouri.

Nous pouvons notamment porter au crédit de Nicolas Adell le fait de vouloir définir l'homme par les savoirs dès son introduction et d'insérer l'anthropologie des savoirs dans le cadre plus large de la «société des connaissances» appelée par un rapport de 2005 de l'Unesco. Il développe cette idée à la conclusion de son ouvrage, intitulée "l'anthropologie des savoirs dans les sociétés du savoir" et il en montre la généalogie par l'auteur conférencier Peter Drucker dès les années 1960 aux États-Unis. L'ouvrage ouvert sur une considération anthropologique (l'homme est celui qui sait) s'achève sur une note sociétale (la société de la connaissance), et son tour d'horizon des sciences humaines et sociales accorde une place de choix à l'histoire, du moins celle de la constitution de champ de recherche. Ce chapitre permet notamment de situer, en référence à son manuel, notre travail dans les ethno-sciences qui se développent avec l'anthropologie cognitive.

Il reste cependant au débit de l'auteur qu'il veut séparer et écarter la technique de la pensée, négligeant ainsi les travaux de Jack Goody sur l'écriture comme technique intellectuelle (ou même avant lui les écrits du philosophe Wittgenstein que Morin utilise

\footnotetext{
${ }^{106}$ Adell Nicolas, 2011, Anthropologie des savoirs, Armand Colin. Collection U. SHS. Paris.
} 
pourtant dans cette optique dans l'ouvrage précité). Au-delà de ces considérations anthropologiques et pour en rester à des objets plus éducatifs, ajoutons qu'il passe sous silence des didacticiens des disciplines importants comme Yves Chevallard ${ }^{107}$ et Guy Brousseau qui, dès les années 1970 ont adopté la perspective anthropologique dans leur didactique des mathématiques, pour forger les concepts de transposition ou encore pour éclairer le contrat pédagogique soutenant la transmission de savoirs ${ }^{108}$.

\section{Au-delà de la notion de métier : les anthropologies mobilisées}

Dans la revue de littérature que nous avons proposée, nos apports sont multiples. Ils ont trait tout d'abord à l'approche par métier qui centre l'intérêt sur les acteurs professionnels et leurs contextes de travail ainsi que les outils pour l'action. Notre anthropologie des mondes contemporains génère des schémas de compréhension dans différents métiers : ainsi les organisations innovantes de la nouvelle économie et leur sociogramme peuvent être comparées à une administration industrielle de l'enseignement à distance pour mieux comprendre les logiques professionnelles à l'œuvre et dépendant du secteur économique - et donc son degré d'incertitude et de risque - dans laquelle l'activité est réalisée.

Notre anthropologie des métiers et du travail éducatif développe ainsi les conceptions d'anthropologie économique de Maurice Godelier ${ }^{109}$, auteur largement reconnu par le Centre national de recherche scientifique, dans son volet «ethnographie des organisations » qui accorde une place de choix à l'analyse de la valeur. Ceci est le cas dès les travaux sur la nouvelle économie où est montrée la socialisation des employés, et se prolonge dans notre déontologie des organisations avec l'acculturation aux valeurs de l'enseignement à distance et les conflits alors générés. Là où l'approche de Maurice Godelier, elle aussi laissant une large place à la notion d'outil et d'usage, produisait des données macro-économiques, notre analyse de la valeur se différencie par une approche méso-économique, au niveau de l'économie institutionnelle observée avec la distance propre à notre discipline.

L’anthropologie économique fondée par Godelier bénéficie ainsi des apports de l'anthropologie politique, plus ancienne en France et remontant aux travaux de Georges

\footnotetext{
107 Voir notamment : http://yves.chevallard.free.fr

108 Notamment Brousseau G., 1980, L'échec et le contrat, Recherches, 41, pp177-182.

${ }^{109}$ Même si ses débuts ont parfois étés difficiles : Godelier M., 1974, Un domaine contesté : l'anthropologie économique, Mouton. France.
} 
Balandier, repris et développés par Marc Abélès ${ }^{110}$ par l'entremise d'une anthropologie des mondes contemporains. Il s'agit en effet d'étudier les institutions comme lieu de pouvoir : le centre d'enseignement que nous avons étudié a, dans les années 1980, été doté du statut d'académie et jouait donc un rôle primordial dans l'innovation pédagogique.

Notre anthropologie du travail éducatif innovant se centre ensuite sur la formation des adultes, ce qui est différent du panorama des recherches évoquées. Le public ciblé appelle une relation éducative différente que pour la pédagogie traditionnelle, moins hiérarchisée et plus centrée sur le concret et l'utilité immédiate dans les expériences partagées. Ce thème, largement développé par les approches andragogiques, est repris et fortifié par observation directe de la production de connaissance par les gens du métier pour les utilisateurs que seront les apprenants.

L'influence de nos travaux doctoraux sur Nietzsche, qui remonte à l'âge frustre de l'anthropologie ${ }^{111}$, nous amène aussi à revoir avec le recul de l'histoire des idées plusieurs notions comme la place centrale accordée à l'apprenant aujourd'hui en Europe, ou encore la notion de valeur éducative au centre des processus d'évaluation et confrontant le quantitatif (qu'il soit docimologique ou économique) au qualitatif (les valeurs des acteurs, attracteurs individuels et collectifs dégagés par les ethno-méthodes). Ce fond philosophique propre à notre thèse de doctorat des universités débouche sur une déontologie en organisation qui se différencie des travaux de l'économie des grandeurs qui avait une approche macro-sociale.

Certaines des idées du Nietzsche éducateur, reprises par Dewey, concourent aux pédagogies actives de l'apprentissage par le faire (learning by doing) et de l'expérimentation, notamment sur le lieu de travail pour les adultes en formation. Notre anthropologie de la formation est donc une anthropologie de l'action formative qui s'inscrit dans le prolongement de notre première thèse : il s'agit là aussi de décrire l'éthique d'un spectacle dont on est parfois soi-même aussi acteur. La déontologie n'est plus celle d'un métier de l'art, réévaluée par les classiques occidentaux (approche homéro-nietzschéenne) mais elle est issue du spectacle de l'action productrice du travail éducatif avec ses dimensions scientifiques des littératures contemporaines en sciences humaines et sociales.

\footnotetext{
${ }^{110}$ Abélès Marc, Jeudy Henri Pierre, 1997, Anthropologie du politique, Armand Colin, collection U. France.

${ }^{111}$ On peut ainsi comparer La généalogie de la morale à La théorie de la classe de loisirs de Veblen : les deux auteurs de formation philosophique font œuvre d'anthropologie en appelant à des comparaisons avec une civilisation primitive qu'ils idéalisent pour critiquer l'ère moderne au $19^{\text {ème }}$ siècle colonial.
} 
De ce fait, l'expérience de la souffrance et de la douleur, typiques du tragique nietzschéen et largement développée dans notre thèse, sont abandonnées du fait de l'influence de Dewey (qui ne place pas le «travail» (fût-il «scolaire») dans son sens premier d'instrument de torture) et la reprise de la question par Olivier Reboul ${ }^{112}$ et ses notions de joie dans l'apprentissage qui renvoient à des processus psychologiques de renforcements positifs peut être plus efficaces que les méthodes anciennes de punition. Nous rejoignons donc les thèses de Jean Marie Barbier que nous synthétisons dans l'expression de « l'épistémophilie anthroponomique » (Entretien filmé par l'université de Rouen entre Jean Marie Barbier et Oivier Marty au Cnam en 2019, à paraître).

Les liens entre les observations (ou théorie) et participations (ou pratiques) sont multiples dans nos travaux (et c'est en cela que l'on peut parler avec Jean Marie Barbier de polysémiotisation de l'action), soit que l'on est participant observateur (terrain dans l'enseignement à distance, entreprises innovantes), soit que l'on observe ses propres participations (groupe de travail en grande école) ou celles d'autres qui nous sont étrangers (ethnographie en Guyane). C'est le degré d'implication dans l'action qui varie, selon la triade des distances identitaires que nous avons décrite en méthodologie.

Notre apport est enfin propre à l'anthropologie des savoirs dans l'architecture des connaissances qui est proposée dans un article issu de notre terrain guyanais. L'influence du professeur Philippe Descola, et notamment de son ouvrage Par delà nature et culture est déterminante puisqu'il s'agit de proposer une épistémologie de l'encastrement des savoirs en tentant d'abandonner nos propres cadres occidentaux de pensée pour épouser la forme de ceux observés sur le terrain. Ainsi un emboîtement à trois niveaux propose de placer le physique à la base, sur laquelle s'appuie le social et dans lequel s'inscrit le psychique. Cette trilogie pyramidale que nous ne détaillerons soulève toutefois la question anthropologique de savoir d'où vient l'idée de l'emboîtement, si c'est une perception pure issue de l'observation de l'ethnie considérée ou si c'est une conception venue du milieu scientifique d'origine. L'expérience anthropologique de l'altérité génère des schémas dont il est difficile de trouver l'origine certaine: est-ce que la distance à sa propre culture occidentale, provoquée par l'acculturation au terrain, permet de contempler de loin sa propre mentalité pour en donner les grandes lignes (cette architecture du savoir que nous proposons) ensuite projetée sur l'ethnie observée en clamant une démarche heuristique ? Ou bien est-ce sincèrement ce qui est

\footnotetext{
${ }^{112}$ Reboul O., 2010, Qu'est-ce qu'apprendre ? PUF, Éducation et Formation. France.
} 
observé sur le terrain en faisant table rase de tous ses schémas cognitifs importés ? C'est là la difficile question de l'équilibre entre conceptions et perceptions sur laquelle nous reviendrons dans le chapitre suivant.

Toujours est-il que nous proposons de qualifier d'anthropologie épistémique cette démarche qui vise à produire des systèmes hiérarchisés de connaissances en se confrontant à d'autres cultures, métiers ou géographiques. Ceci prolonge le programme de travail sur « la métaphysique des autres » de l'anthropologie de la nature du professeur Philippe Descola au Collège de France, reprise en partie en dialogue avec notre directeur de thèse Francis Wolff à l'École normale supérieure, dans son séminaire de philosophie hebdomadaire, sous son volet métaphysique. Ce que nous apportons est un traitement de ces questions en anthropologie des mondes contemporains, en provoquant la réflexion au sein de communautés scientifiques de spécialistes centrés sur l'éducation occidentale.

Ainsi notre contribution d'anthropologie épistémique est d'abord une anthropologie des savoirs (et de leurs encastrements), mais elle touche aussi à l'anthropologie de l'éducation dans le sens où nous avons travaillé le triangle professeur-savoir-élève, et donc les questions de relations éducatives et de conditions sociales de production ou de consommation de connaissances. Comme on le voit dans le schéma ci-dessous, on peut constater qu'il ne s'agit plus d'un triangle équilatéral mais d'un triangle isocèle du fait de l'écartement entre enseignants et enseignés, séparés par les média qui véhiculent l'enseignement.

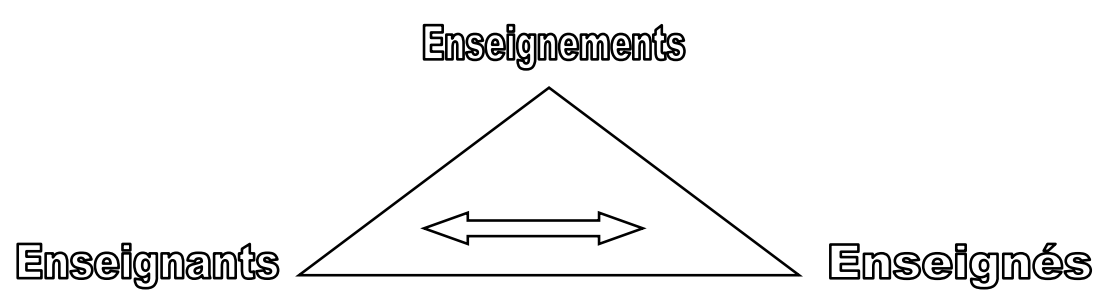

\section{L'aplatissement de la triade houssayenne dans l'enseignement à distance}

Du fait de la médiatisation des enseignements qui écarte les enseignants des enseignés

Dans cette triade ainsi retravaillée pour être élargie, on a pu apporter différents éléments novateurs comme l'analyse de la relation éducative médiatisée enseignantsenseignés, les dynamiques d'apprentissages en ligne des enseignés (notamment en phase de 
construction de savoirs), les organisations de la production ou de la diffusion des enseignements par les métiers enseignants - ce qui nous a amené à utiliser des cadres d'anthropologie économique et de sciences de gestion (partir des demandes de savoir pour déterminer la production, organisée par un mode gestionnaire), nous l'avons vu, mais aussi des sciences de l'apprendre psychologisantes (Bandura, Bloom, etc.). La notion de rapport au savoir a été transférée des enseignés vers les enseignants en observant non plus les inhibitions culturelles mais au contraire les aspects facilitateurs de la profession. Enfin nous nous sommes intéressés à la politique de l'éducation et des enseignements au travers de l'ingénierie didactique d'un programme de philosophie pour voie professionnelle, questionnant ici aussi le rapport au savoir de ces publics-là et l'adaptation des enseignements du fait du lien particulier formation-emploi dans la société de la connaissance (instrumentée et non pas l'apanage d'une classe de loisirs).

La triade houssayenne aplatie permet de dénoncer les dangers de la médiatisation qui objectivent les enseignements (ou savoirs) en donnant l'illusion qu'ils peuvent être extraits de la relation formative. En effet, en constant que le lien enseignant-enseigné est distendu, on voit que les enseignements peuvent être détournés dans leurs interprétations, traverser les siècles pour être réinterprétés ou changer de continent pour des réappropriations qui n'étaient pas prévues. Cette conséquence de la distance permet de pointer les effets de catachrèse des enseignements par les enseignés qui n'étaient pas nécessairement prévus dans les intentions premières des enseignants. La tâche propre à une anthropologie étant alors de remettre en valeur la base humaine enseignants-enseignés du triangle et de rappeler ce fondement nécessaire aux enseignements médiatisés qui auraient tendance s'extraire de ce cadre.

Notre objet premier de réflexion est bien les connaissances ou savoirs, notamment par une analyse soit linéaire (la formation initiale continuée) soit cyclique (la réinitialisation de la formation par reprise d'études) du rapport au savoir. Nous avons ainsi pu travailler l'importance de la première formation pour définir ce qu'est un savoir, quand, où, et comment il est appris (mais par «pour qui », qui reste un impensé pourtant primordial dans la voie professionnelle et ses débouchés), et voir comment la formation d'adultes pouvait soit continuer cette première forme, soit la réinitialiser. L'insertion dans la relation éducative permet de mieux situer ces processus et de voir les acteurs à l'œuvre dans l'apprentissage.

Ainsi, notre anthropologie est plus qu'une science de l'homme se référant aux langues anciennes (sans être assez classique pour être pleinement humanité), elle ne s'oppose pas simplement aux langues modernes bien vivantes des codes informatiques (les humanités 
numériques; comme le «qui ?» (quali) s'oppose au «combien ?» (quanti) dans le recueil de données), notre anthropologie vise à comprendre l'homme dans son rapport au monde tel qu'il le connaît ou s'efforce de le connaître au travers des savoirs. Le fil de nos apports nous amène donc à proposer un chapitre épistémologique sur le lien, entre les «savoirs » et le « réel », complétant le double lien entre «savoirs » et : «professeur » d'une part, « élève » d'autre part.

\section{Une épistémologie équilibrée entre constructivisme et positivisme}

En développant nos apports à l'anthropologie par la notion de métier, nous avons montré l'importance des savoirs pour mieux comprendre la relation éducative. L'architecture des savoirs nous a amené à questionner l'effort épistémologique pour produire ces données. Nous voulons à présent développer notre contribution à l'épistémologie dans deux souschapitres complémentaires : d'une part l'atout d'une formation de philosophe pour penser l'équilibre entre conceptions et perceptions dans tout effort ontologique proprement scientifique; d'autre part les apports d'une situation de praticien pour comprendre les déterminants de l'action d'une praxéologie - chacun de ces deux thèmes faisant aussi l'objet d'une littérature abondante.

\section{Apports épistémiques d'une formation de philosophe}

Nous sommes certes redevables à Nietzsche, mais surtout à Jacques Derrida, pour l'effort que nous présentons de déconstruire le dilemme entre constructivisme et positivisme logique. En effet, plutôt que de philosopher «à coups de marteau » pour détruire l'opposition, nous voulons défaire pierre par pierre l'édifice et montrer que la césure n'est pas nécessaire comme elle est souvent présentée. Le chiasme entre la découverte scientifique d'une réalité existante (par pure perception qui est ensuite conceptualisée) d'un côté ; et l'invention de toutes pièces par les constructeurs de la réalité (qui conçoivent le réel dans les institutions dédiées pour ensuite en trouver une perception, peut être artificielle) de l'autre ; nous paraît par trop grossi lorsqu'il est demandé de se ranger dans un camp épistémique contre l'autre. 
Pour prolonger les propos d'Edgar Morin citant le neurologue Jean-Pierre Changeux ${ }^{113}$, tout en actualisant cette perspective par la consultation de travaux plus récents dans les deux disciplines, il apparaît qu'une anthropologie biologique de la connaissance doit passer par la double reconnaissance des concepts et percepts et l'importance de trouver un juste équilibre entre les deux tendances au lieu de ne considérer qu'un des deux aspects. Puisque la cognition humaine accède au réel en passant à la fois par des conceptions issues en partie du travail mental individuel mais aussi et surtout de la culture éduquée, notamment par le langage; et à la fois par les perceptions sensorielles individuelles (éventuellement augmentées par un dispositif anthropotechnique), il apparaît que l'effort scientifique collectif pourrait reconnaître l'importance égale des deux démarches.

Autrement dit, l'expérimentation perceptive pure n'est rien sans conception préalable (il n'y a pas de réel brut s'offrant à nos sens et donnant lieu à des théories scientifiques falsifiables en s'y référant) tout comme la théorisation pure (mathématiques ou spéculative comme on sait depuis Bloor et malgré Berkeley) semble creuse sans recours aux données du sensible (fût-ce par la transposition des théories pour application dans une autre discipline comme la physique par exemple). Pas de percept sans concept (d'expérience sans question), ni de concept sans percept (de question formelle sans matière). Ceci pourrait paraître une évidence ainsi énoncé mais cet équilibre délicat permet de poser un regard critique et conciliateur sur des débats encore vifs. Il permet aussi de retrouver l'équilibre aristotélicien entre la forme (ici les conceptions) et la matière (ici les perceptions) prenant le nom classique d'hylémorphisme.

Il est certes possible de reconnaître des personnalités, ou des âges, ayant une tendance à la conception ou à la perception, pouvant donner lieu à des prises de positions radicales et des programmes épistémologiques partisans. Il est aussi possible de repérer des cultures disciplinaires donnant plus de place ou bien à l'expérimental ou bien à la théorisation dans lesquelles telles ou telles personnalités trouveront à s'épanouir pleinement - pour reprendre l'idée d'encastrement du psychique dans le social dans notre architecture des savoirs.

\footnotetext{
${ }^{113}$ Edgar Morin dans l'ouvrage sur la connaissance de la connaissance précité (La Méthode, 3) cite Jean Pierre Changeux de l'homme neuronal, ouvrage de vulgarisation en neurosciences dont nous sommes également très redevables malgré certaines propositions qui ont été dépassées mais que nous ne retenons pas ici.
} 
Alexandre Koyré ${ }^{114}$, il y a près d'un siècle, avançait des propositions que nous pourrions qualifier d'une approche anthropologique proche de la nôtre en avançant que les métiers scientifiques dans l'ensemble étaient plus proches de la conception pure alors que les métiers techniques étaient tournés vers la perception du réel. De cette opposition qui pourrait paraître trop binaire et tranchante à première vue, il en tire des conséquences fines sur la difficulté de vouloir confirmer la science par une réussite technique, puisque la multiplicité contradictoire des conceptions des uns est extrêmement appauvrie dans l'approche technique des autres - qui s'appuie sur quelques conceptions enseignées et largement divulguées pour ensuite trouver des solutions expérimentales originales basées bien plus sur leurs atouts perceptifs que leur richesse conceptuelle.

La place de l'instrumentation scientifique est alors centrale puisque c'est elle qui permet de matérialiser une conception (voire plusieurs) et qui sert alors aux techniciens pour percevoir le réel (avec toutefois des possibilités de détournement de la théorie initiale pour mieux s'emparer des réalités qui se présentent). Ces écrits, auxquels nous adhérons en partie permettent de comprendre les enjeux de pouvoir autour de l'instrumentation qui sert de légitimation d'une théorie pour les scientifiques (toutes les conceptions ne sont pas matérialisées par un instrument) et de droit de regard sur le réel pour les expérimentateurs (tous n'ont pas accès à l'instrument qui autorise la perception du réel).

Cette approche dans le sens de Koyré rappelle l'importance de la hiérarchie des faits. Car dans l'infinité perceptuelle c'est le pouvoir d'accorder le primat a telle perception plus qu'à telle autre (en la plaçant au fondement d'une conception) qui ordonne la description du monde. Ainsi la légitimité du scientifique permet de passer de l' «observation» à la «théorie » (étymons désignant sensiblement la même approche visuelle mais aux acceptions scientifiques et courantes relativement éloignées et hiérarchisées socialement)

Cette approche nous amène aussi à une position ontologique du primat de la matière sur la forme. Ce parti affirme que la nature est première et que c'est en son sein que l'homme a commencé à la représenter. Ainsi les idées des sciences de la nature sont humaines (comme les anciennes idées des divinités et celles qui sont discutées et donc discutables de big bang, les concepts de force ou de cause, l'apparition de la vie), elles sont imparfaites et amenées à être perfectionnées. L'anthropologie des savoirs place en premier ce qui doit être su (le réel,

\footnotetext{
${ }^{114}$ Koyré A., 1985, Études d'histoire de la pensée scientifique, Gallimard. Tel. France. Voir notamment le dernier chapitre. Ces propos sont complétés par l'ouvrage réédité en 1988 dans la même collection : Du monde clos à I'univers infini.
} 
auquel l'homme n'a qu'un accès limité par sa perception conceptualisée) mais dont on ne sait rien de définitivement satisfaisant, puis en second l'homme dans son effort pour savoir (c'est le propre des travaux d'anthropologie de la connaissance que nous avons énumérés dans notre revue de littérature) et donc en troisième et dernier toutes les constructions appuyées sur les découvertes que sont les disciplines scientifiques (le monde représenté légitime, a une date donnée des débats).

Nous avons donc une nouvelle pyramide des savoirs avec à son sommet inaccessible le monde nouménal kantien ou l'ontique qui est plus qu'un être linguistique puisqu'il est objet d'expérience. L'anthropique intermédiaire désigne les efforts humains pour connaitre cette ontique. Par un ensemble de perceptions et conceptions s'élaborent des ensembles épistémiques qui peuvent alors être étudiés par les sciences humaines (histoire, sociologie, ethnologie des sciences). Mais la base principale de la pyramide reste les ensembles scientifiques traditionnels, ici nommés épistémiques : les théories des différentes disciplines (nous nous limitons en France à celles que reconnaissent le CNU et le CNRS dans leurs sections et prenons un sens large incluant médecine, droit et théologie au-delà des sciences et lettres). Pour retrouver l'hylémorphisme, on ajoute une tension entre la matière ontique en haut de la pyramide et les formes humaines en bas. 


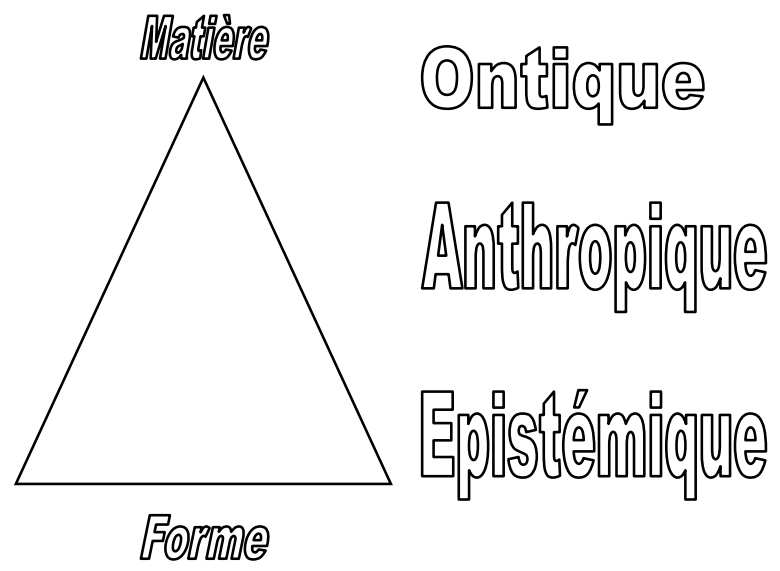

\section{La pyramide des savoirs}

L'anthropologie épistémique est une phénoménologie réflexive : une science de comment l'être nous apparaît

Si l'on ne peut quasiment rien savoir de définitif sur la matière telle qu'elle est (bien que ce soit vers elle que se tournent tous nos efforts pour savoir), l'anthropologie des savoirs a une première place épistémologique par défaut pour comprendre la connaissance en action, mais elle s'efface enfin et rapidement derrière toutes les connaissances disciplinaires qui présentent des représentations légitimes, formalisées et pertinentes de la matière. L'anthropologie épistémique est comme un intermédiaire, passage obligé pour comprendre les interactions du couple matière-science, ou encore réel-savoirs humains : ce couple vit dans des relations de va et vient et ce sont ces mouvements qu'étudie l'anthropologie des savoirs. Elle n'étudie pas que l'acte de recherche sur le réel mais s'intéresse aussi, comme nous allons le voir, aux processus de diffusion des connaissances (par exemple par les métiers de l'enseignement à distance) et les processus d'acquisitions individuels de non spécialistes.

Si elle s'inscrit dans un matérialisme naturel, elle traite les savoirs comme humains et rien qu'humains (au sujet de choses qui ne le sont pas nécessairement, comme la physique). Elle ne prétend pas que tout est humain : il existe certes un réel premier dont l'ontologie est impossible mais aussi des représentations humaines dont on peut étudier la production et diffusion par les professionnels. On ne choisit pas l'anthropique contre l'ontique : l'ontique est première mais inaccessible ; bien que l'expérience instrumentée amplifie la perception et les sciences purifient les conceptions. 
La place des ethno-sciences, que nous évoquions via notre revue de littérature, est alors importante : si «nos » sciences d'une « grande société ouverte » permettent d'habiter un vaste espace formel de réalités scientifiques et techniques dans la matière première, conforté par le miracle quotidien des techniques, il est important d'étudier les efforts similaires d'autres populations pour se construire un monde de représentations, et le traiter sans porter de jugements appuyés sur des valeurs issues de notre propre monde (notamment à l'aune de nos prouesses techniques que nous interprétons avec notre propre référentiel scientifique et non l'appareil conceptuel de l'ethnie étudiée). Les travaux de Philippe Descola montrent ici comment leur propre physique et leur propre métaphysique leur permet d'élaborer des savoirs qu'ils jugent pertinents sur la nature.

Notre apport que nous proposons, nous inscrivant dans les science studies ${ }^{115}$ quoique les prolongeant jusque dans la réception des connaissances par les individus par l'intermédiaire de professions du savoir, est de pousser la logique des ethno-sciences jusque dans l'anthropologie des mondes contemporains : l'idée d'un monde scientifique unique et unifié valable pour tous les occidentaux - c'est-à-dire consensuel dans la communauté d'experts et diffusé avec homogénéité par la vulgarisation scolaire et médiatique, donnant enfin lieu à une réception équitable par chaque individualité - semble en effet une utopie et les ethno-méthodes au sein de notre propre société amènent à avancer l'idée de mondes pluriels plus ou moins homogènes. La place de l'anthropologie des savoirs étant alors aussi d'étudier l'homme dans l'action de connaître, non pas seulement comme scientifique pour toute une société (étudiant les sociétés savantes et leurs logiques d'imposition de sens), mais comme groupe social restreint habitant tel espace particulier (du fait de sa profession par exemple) voire comme seul individu déterminé face aux informations dont il dispose (et son caractère propre issu d'un développement idiosyncratique).

On peut alors placer dans ce panorama notre recherche sur les métiers de l'enseignement à distance. En indiquant par exemple le rôle de diffusion à vaste échelle de connaissances scientifiques choisies (via les programmes de préparation à l'agrégation ou les licences et masters à distance que propose l'institution étudiée à plusieurs centaines de milliers d'apprenants), tendant à homogénéiser et diffuser le monde scientifique représenté dans les différents groupes. Le métier de l'enseignement à distance, dans son aspect pédagogique, a alors un sens comme passeur et diffuseur de connaissances - ce qui est vrai plus largement des métiers de l'éducation.

\footnotetext{
${ }^{115}$ Voir par exemple : Hacking I., 2000, The Social Construction of What ?, Harvard University Press.
} 
Ce qui amène à préciser que nous complétons les recherches sur la recherche en train de se faire (Latour, Bloor par exemple) par des données sur les diffusions de la recherche via l'appareil éducatif - avec les particularités de l'enseignement à distance, centralisé par cette institution en France jusqu'à encore récemment (elle subit depuis une quinzaine d'années de plein fouet la concurrence des différentes universités produisant de l'enseignement à distance, ce qui donne lieu à un appareil de diffusion de grande échelle et industriel pluri-centré). Là où le centre régnait seul sur l'enseignement à distance francophone et avait un pouvoir sur les connaissances diffusées via les choix de partenariats vers telle ou telle université ou école dont il assurait la grande audience, il est à présent concurrencé par d'autres institutions diffusant directement leurs propres recherches. Il est encore remarquable que le comité pour l'élaboration des programmes du secondaire se réunisse dans le même bâtiment que l'institution, trace de cette centralité passée où se décide encore pour beaucoup se qui formatera le monde de représentations dans lequel vivront les nouvelles générations suivant les programmes dans leur scolarité obligatoire.

L'idée même d'un «centre » autour duquel tout tournerait, pivoterait ou verserait est d'ailleurs propre à l'étymologie de l'université ${ }^{116}$, concept datant d'une conception de l'univers concomitante de l'apparition médiévale de ces institutions - c'est ce que nous avons présenté à l'université d'Aarhus au Danemark, pour un congrès en 2017 sur l'avenir de l'université. Plusieurs auteurs débattent encore de savoir si c'est une conception française de corporation de maîtres et d'élèves qui graviteraient ensemble ou bien une conception allemande de savoirs tournant autour d'un centre. La question étant alors évidemment de savoir la nature de ce centre. Au-delà des bâtiments et de l'architecture propre des lieux de gravitation - qui n'ont pas toujours été prestigieux et qui ont changé dans l'histoire de chaque institution, s'écartant progressivement des édifices religieux - les savants de l'époque, tel Saint Thomas d'Aquin mais aussi Al Ghazali dans les équivalents des civilisations d'outreMéditerranée, avancent souvent une idée dont la polysémie est reconnue par les philosophes

\footnotetext{
${ }^{116}$ Cf. la littérature sur l'histoire sociale et la philosophie des universités, dont :

- $\quad$ Durkheim Emile, 1938, L'évolution pédagogique en France, Paris ;

- Renaut Alain, 2015, Les Révolutions de l'université. Essai sur la modernisation de la culture, Calman Levy. France. qui prolonge Gusdorf George, 1964, L'université en question, Payot. France.

- $\quad$ Repris récemment par les historiens : Gilli P., Verger J., Le Blévec D., 2007, les universités et la ville au Moyen-âge. Cohabitation et tension. Leiden, Boston, et la synthèse dans Christophe Charle et Jacques Verger Histoire des universités XII e XXI e siècle 2012, Puf. Quadrige manuel. France.

- Ou encore l'équivalent pour une autre aire culturelle : Dodge Bayard, 2011 (1961), Al Azhar, one millenium of muslim learning, Literacy Licensing, The middle east institute.
} 
contemporains laïques ${ }^{117}$ : le monothéisme. Un concept de dieu si fédérateur (le «toutpuissant », l'« unique », le «clément », le «miséricordieux »... tel que le décrit la tradition scolastique, catholique autant que musulmane) permet en effet de rassembler un grand nombre de notions et a longtemps été placé au centre des mondes représentés.

Aujourd'hui, toutefois, notre perspective d'anthropologie des connaissances nous amène à nous détacher d'une idée d'un centre unique ou d'une idée ultime qui serait l'axe de pivot de tout un système de pensée. Le fait que plusieurs mondes scientifiques cohabitent, voire même que les physiciens débattent d'un «multivers » pluri-centré en lieu et place de l'univers, nous amène à relativiser toute idée de centralité définitive. La vision de réseaux scientifiques avec plusieurs points de convergence remplace la forme étoilée avec un centre rayonnant, ce aussi bien socialement, dans la constitution des espaces de débats scientifiques, que scientifiquement dans les mondes de représentations élaborés (la seconde proposition dépendant peut-être de la première).

Ceci n'écarte cependant pas tout l'apport des discours religieux, qui sont à prendre avec leur sens étymologique (qu'il s'agisse du latin, du sanskrit, de l'arabe...). Ainsi le débat encore très actuel sur les croyances scientifiques et les prises de position pas toujours raisonnées renvoie à l'adhésion affective, au cœur (c'est une des racines majeures du mot croire) mis à l'ouvrage scientifique qui peut expliquer les passions et les émotions dans les débats. Comme nous l'avons esquissé dans notre didactique de la théologie (étude sur les techniques de formation des cadres religieux dans une institution monothéiste), qui se veut être une étude d'anthropologie de l'éducation (par une approche ethnographique dans une organisation éducative financée par l'état français), les pratiques anciennes de ces institutions sont à replacer dans leur contexte culturel, linguistique et historique de création et perdent parfois de leur sens face à la modernité, notamment lorsque l'on constate l'évolution de la langue (quoiqu'un grand effort soit fait pour les adapter en permanence).

Et nous finirons ce chapitre d'épistémologie dans son volet philosophique en évoquant les travaux de Jacques Bouveresse ${ }^{118}$ - philosophe français voulant user de la méthode scientifique après avoir renoncé à la prêtrise - portant sur la notion de vrai et de faux. Il montre en effet que les croyances et les principes affectifs sont importants dans l'effort

\footnotetext{
117 On peut comparer, dans notre bibliographie, deux auteurs contemporains du Collège de France : Rommer de L'invention de Dieu et Jacques Bouveresse de Peut-on ne pas croire, ils prolongent les débats philosophiques des années 1960 sur " l'idée de dieu »

118 Notamment : Bouveresse J., 2007, Peut-on ne pas croire : sur la vérité, la croyance et la foi, Agone. France.
} 
scientifique et peuvent contribuer à expliquer la remise en cause contemporaine de la notion de vérité. S'appuyant sur l'influence déterminante de Nietzsche (sur Musil, Wittgenstein et le cercle de Vienne), il montre que cette idée de vrai, tout de même fort utile pour fédérer les communautés et prouver par utilité en finalité la pertinence de telle ou telle conception, est dépendante d'une certaine sentimentalité (l'esthétique du vrai: la beauté, la simplicité, l'harmonie, la justesse, etc.) qui peut être collective. Ainsi l'anthropologie des savoirs accorde selon nous - et dans le prolongement de notre second chapitre de thèse de doctorat des universités - une place importance à l'esthétique scientifique, individuelle ou collective, comme principe de validation de connaissance basé sur des données purement humaines comme l'émotion ou le sentiment - en-deçà de leur rationalisation.

\section{Perspectives épistémiques ouvertes par la pratique}

Notre apport épistémologique dans l'anthropologie des savoirs ne serait pas complet si on n'énonçait pas une autre partie de ses conditions de production et leur caractère positif pour la recherche. Nous avons montré l'environnement de lectures philosophiques et scientifiques qui introduit à des courants de pensée qui vont souvent puiser à la source grecque des idées à régénérer et interpréter dans l'actualité de chaque discipline moderne (histoire, sociologie, ethnologie de l'éducation). À ce tour d'horizon des lectures et débats auxquels nous avons été sensibilisés, il faut ajouter la dimension pratique de notre formation. En effet, notre effort pour doubler notre formation à la recherche par un master de grande école à visée professionnelle, et la suite qui a consisté à décrire de l'intérieur les métiers de l'enseignement à distance dans leur dimension gestionnaire, donne lieu à ce qu'il est convenu d'appeler depuis L. V. Mises une praxéologie, ou théorie de la pratique.

Les méthodes diverses d'observation-participante, de recherche-action, d'ethnographie se sont combinées pour trouver un lieu d'expression durable en tant que chercheur affilié au Conservatoire national des arts et métiers (connu pour cette alliance entre la pratique et leurs théories) et nous voulons à présent donner l'apport conceptuel de ce positionnement.

Le premier apport épistémologique est la possibilité de compléter les conceptualisations venues de la littérature et des séminaires de recherche par des observations pratiques, des perceptions directes en situation (dans les métiers) qui complètent l'effort de 
connaissance. Ces perceptions ont fait l'objet de carnets de terrain ethnographique et d'écriture réflexive hebdomadaire pour en garder la trace permanente et pouvoir revenir sur ces expériences pour les réinterpréter - sans perdre toute leur richesse pleine de réel - à l'aune de cadre conceptuels et théoriques.

Nous pouvons d'ores et déjà évoquer l'apport important d'Yves Clot et de sa clinique de l'activité qui amène à considérer non pas uniquement l'action réussie, mais aussi et symétriquement l'action qui a échoué et les intentions non abouties. Ainsi une partie de analyses porte sur des projets d'ingénierie de formation à distance qui n'ont pas été réalisés jusqu'au bout, souvent pour des raisons économiques. Comme Bruno Latour étudie les entreprises scientifiques réussies symétriquement de celles qui ont échoué, et nous avions repris ce principe épistémologique dès notre travail de recherche sur la nouvelle économie et ses Start Up connaissant le succès financier ou la faillite, nous pensons qu'il est important de considérer les moyens également et indépendamment des fins atteintes.

D'autant plus, et c'est un apport de notre anthropologie, que les moyens mis en œuvre débouchent parfois sur des fins différentes de celles visées et sont alors réinterprétés pour servir ces nouvelle fins. Étudier l'action à un temps T, avec ses reconstructions historiques et ses projections futuristes, peut se faire indépendamment de la validation de cet état comme moyen d'une fin située au temps $\mathrm{T}+1$. Chaque moment est indépendant et comporte son passé et son futur, qui seront différemment cités et réécrits au moment suivant. On peut donc échapper au jugement du moment premier par le moment second et étudier, par gros plan dans l'action, toutes les dimensions d'un moment unique. L'action est alors prise dans unité perceptive et conceptuelle la plus fine possible.

Notre praxéologie, du fait de ses gros plans sur l'action, débouche ainsi sur une conception «présentéiste» du temps, où chaque moment discret inclut son propre passé et son propre futur que l'on va étudier comme lui appartenant. Ceci au lieu de se centrer sur les continuités entre moments comme a par exemple plus le faire Ricoeur dans ses essais sur la narrativité temporelle et la linéarisation par des récits. Cette seconde conception, plus commune, pourrait être développée dans une rétrospection globale ne profitant pas de la richesse des carnets de terrain et de l'écriture scientifique régulière.

Nous avons écrit, dans le premier chapitre introductif, nos premiers apports à une anthropologie de l'action, des savoirs en action et des acteurs du savoir. En évoquant les différentes dimensions de la poétique (action créatrice), le drame (action sociale), la praxis 
(action finalisée) et l'ergologie (étude de l'action productrice), nous avons présenté nos propres apports au travers des résultats de plusieurs publications et avons ensuite débattu du lien entre science et action: le point de vue de l'acteur situant sa pensée dans le cours des événements (temporalité, finalités, technicités, configuration...) et produisant des énoncés, non pas au service de l'action, mais libérateurs et validés par des instances externes dont l'objet de recherche est le cadre de cette action.

Cette seconde action, action scientifique dans l'action éducative, est une action réflexive, dont le reflet ne fait pas miroiter de meilleurs résultats mais est discuté par les chercheurs, écrit dans ses formes approuvées et se veut être fidèle à « la réalité » (avec toutes les nuances apportées par notre chapitre précédent à ce sujet). C'est une ontologie scientifique qui dit ce qui est - voire ce qui a été et sera peut-être - et non ce qui devrait être. À cet égard, les valeurs et leur descriptif, qui ont une place importante dans nos analyses, sont des objets scientifiques et non un fondement prescriptif.

Un apport épistémique important sur les conditions de cette praxéologie se situe dans l'éthique de l'enseignement et la recherche, ou comment concilier les mœurs et déontologies parfois contradictoires entre la science des métiers de la distance d'une part; et le terrain éducatif d'autre part. Si les mœurs divergent surtout quant aux usages de la vérité (la place de la critique, du débat, la visée d'une production d'énoncés valides sur le temps long sont des attraits du monde anthropologique), les déontologies renvoient à des référentiels de valeurs relativement différents (l'efficacité et la valorisation des travaux qui sont présentes pour les anthropologues comme pour les indigènes mais sous des formes différentes un vocabulaire propre et visant des publics distincts).

Un conflit d'appartenance peut poser des questions d'identités contradictoires comme nous l'avons montré, mais la didactique professionnelle de la recherche nous pousse à avancer une ambivalence, permanente ou alternative, qui permet de faire valoir des actes dans l'un ou l'autre monde et d'obtenir, au mieux, des doubles reconnaissances (quoique souvent différées). La liberté du chercheur est qui plus est accrue du fait de sa pluri-appartenance institutionnelle qui impose plus de contraintes, le stimule et le pousse à innover en permanence.

Les métiers de l'enseignement (et leurs cadres gestionnaires) et de la recherche, parfois considérés comme distincts, parfois alliés comme dans le statut d'enseignantchercheur au trait d'union qui peut prendre plusieurs manifestations réelles, mobilisent des 
arts ou compétences relativement homogènes. Notamment eu égard à l'appareil de production verbal et scriptural commun et les instances académiques de légitimation et de reconnaissance de la qualité. Par exemple, telle institution qui est source de connaissance à diffuser pour les enseignants est aussi lieu de production de savoir pour les chercheurs. Ce qui indique qu'une anthropologie des métiers est plus facile à réaliser dans ce champ-là du fait de ces proximités que dans d'autres mondes plus éloignés de la science.

La professionnalisation à l'anthropologie des métiers de l'enseignement à distance, ou formation des nouveaux chercheurs à ce champ disciplinaire et à ses objets, est donc complexe et, au-delà des avantages initiaux des chercheurs hybrides («halfies »), elle peut commencer relativement tôt par une socialisation inchoative aux deux types de valeurs et techniques dès l'entrée dans le supérieur. Le développement de la formation tout au long de la vie laisse entrevoir des possibilités de former sur le temps longs des professionnels à une démarche d'analyse de l'action et de recherche sur leurs activités qui n'étaient pas aussi évidentes pour les générations passées. 


\section{Index des notions}

acte, 191

action, $28,58,62,65,68,78,101,116,139$, $140,141,142,143,144,146,147,153$, $169,170,172,174,175,177,179$

administration, $7,31,42,43,44,45,47,55,70$, $96,97,145,172,174,182$

anthropologie, 6, 8, 9, 10, 12, 42, 73, 103, 113, $120,145,177,178,179,180,181,182,183$, $184,185,186,187,188,189,191,192,194$, 195, 196, 198, 201, 205, 206

apprentissage, 6, 7, 13, 22, 24, 25, 28, 31, 36, $37,38,45,63,87,101,109,121,129,132$, $136,141,143,144,145,146,147,148,152$, $156,157,158,161,166,175,179,181,183$, $184,186,203,210$

communication, $7,32,50,56,68,87,99,103$, $117,123,125,130,165,167,181,201,202$, 207

conception, $7,8,10,34,35,36,39,52,56,57$, $60,61,70,71,75,76,77,79,80,81,83,85$, $86,88,89,90,92,125,177,181,184,188$, 189, 193, 195, 196, 208

déontologie, 182, 183

valeur, 25, 26, 27

didactique, 182, 186, 194, 197

discours, 194

distance, 9, 11, 13, 182, 184, 185, 186, 191 , 192, 193, 195, 196, 197, 198, 210, 211

ethnologie

ethnographie, 190, 195

expérience, 10, 13, 184, 188, 190, 191

formation, $11,12,13,179,180,183,186,187$, 194, 195, 196, 198, 201, 204, 207, 208, 211 gestion

management, $7,8,21,22,29,35,41,42$, $43,48,49,51,52,54,55,56,57,58,61$, $62,63,64,65,68,70,73,74,76,78,92$, $100,113,121,122,123,124,125,173$, $186,206,208$

ingénierie

ingénieur, 8, 11, 12, 15, 16, 21, 22, 24, 25, $26,27,28,34,35,36,41,45,51,52,55$, $59,61,70,71,72,73,74,75,76,77,78$, $79,80,81,82,83,84,85,86,87,88,89$, $90,91,93,96,97,98,103,105,109,120$, $123,124,125,151,172,186,196,202$, 208

institution, 11, 13, 192, 193, 194, 198, 201

média, 13, 185

métiers, 11, 13, 180, 182, 185, 186, 189, 191, $192,195,197,198$

numérique, 207

organisations, 10, 13, 182, 186

outils, 13,182

pédagogie, 183, 207

pratiques, 184, 194, 195, 203

professionnalisation, 198, 204

sens, $10,184,185,188,189,190,192,194$, 203

université

universitaire, 193, 205, 210

valeur, 13, 182, 183, 186 


\section{Table des illustrations}

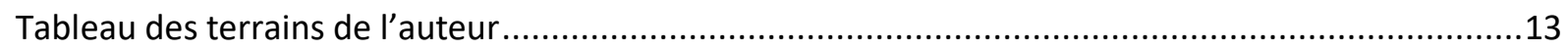

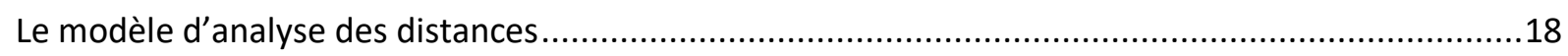

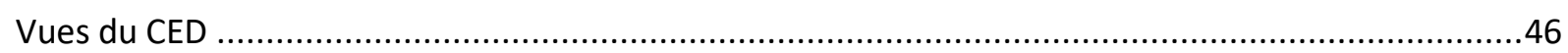

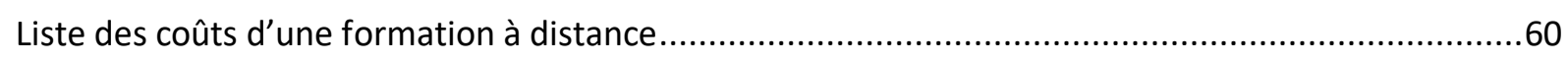

Un exemple de site web de formation produit par l'institution ....................................................71

Exemple d'actant contenant les gestes des acteurs : le forum d'un site de formation ......................73

Aperçu de la fiche de conception du nouveau produit de formation (sommaire du document) .........76

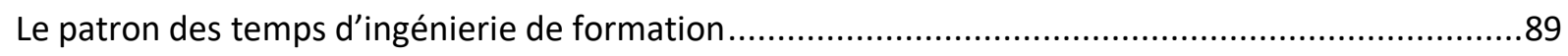

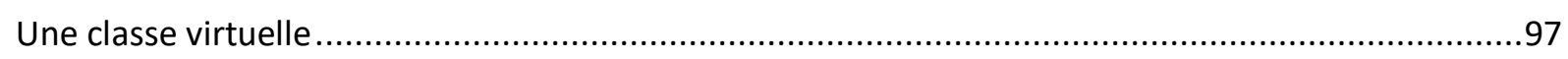

Le sociogramme comme mémoire collective structurante ........................................................101

Le cadre présent des actants non humains : la force des choses...............................................103

Les stratégies individuelles pour le futur : entre alliances et divergences .......................................106

Le modèle coopératif de l'algèbre synchrone ..........................................................................108

La dynamique coopérative : algèbre diachronique de trois moments successifs ...........................110

Les simplifications de la coopération : quand le présent déteint sur le passé et le futur ..................111

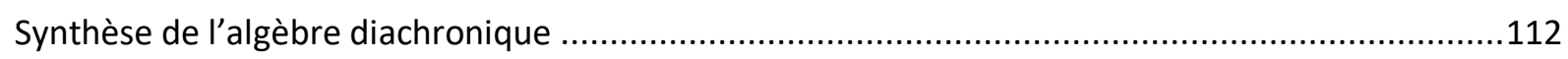

Savoir médiatisé et intermédiaires éducatifs ............................................................................118

Le droit chemin : jeux d'échelles sur une méthode...................................................................142

Types didactiques

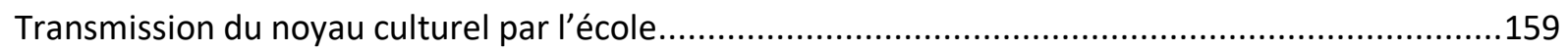

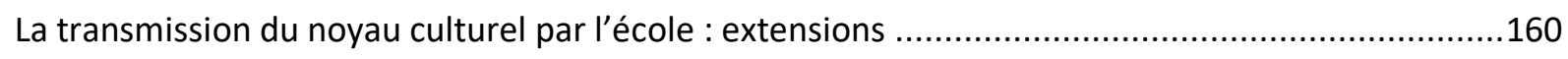

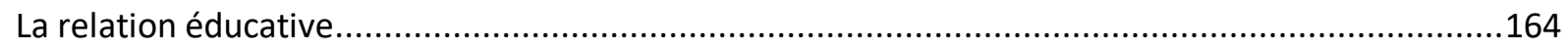

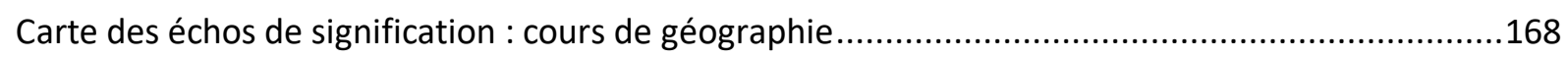

L'aplatissement de la triade houssayenne dans l'enseignement à distance ..................................185

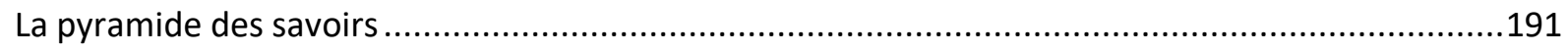




\section{Bibliographie d'anthropologie des organisations éducatives}

Abélès M., Jeudy H.P., 1997, Anthropologie du politique, Armand Colin. Collection U. SHS. Paris.

Adell N., 2011, Anthropologie des savoirs, Armand Colin. Collection U. SHS. Paris.

Astier P. (dir.), 2003, « Analysons nos pratiques », Les cahiers pédagogiques, dossier n416. France.

Alain E.A.C., 1986 (1932), Propos sur l'éducation - Pédagogie enfantine. PUF. Quadrige Grands Textes. Paris.

Albero B., 2001, Pratiques d'apprentissages dans et hors institution : une dialectique enfin possible dans les dispositifs émergents de formation. Recherches en communication, 2001, Médias, éducation et apprentissages, p.103-119. <edutice-00000195>.

Augé M., 2010, Pour une anthropologie des mondes contemporrains. Flammarion. Champs Essais. France.

Bachelard G., 1938, La formation de l'esprit scientifique. Vrin. Paris.

Bachelet R., Delpeyroux S., 2015, Intégrer un Mooc dans un cursus de formation initiale, p.61-70, actes du $8^{\text {ème }}$ colloque «Questions de pédagogie dans l'enseignement supérieur: Innover, comment et pourquoi ? », Ensta Bretagne, telecom Bretagne, UBO. France.

Bakhtine M., 1984. Esthétique de la création verbale. Gallimard. Paris.

Balandier G., 2013, Anthropologie politique. PUF. Quadrige. France.

Barbier J.M., 2011, Vocabulaire d'analyse des activités. PUF. Formation et pratiques professionnelles. France.

Barbier J.M. (dir.), 2011, Savoirs théoriques et savoirs d'action. PUF. Éducation et Formation. France.

Barbier J.M., 2010, « Cultures d'action et modes partagés d'organisation des constructions de sens », Revue d'anthropologie des connaissances, Vol 4, n 1 .

Barbier J.M. (dir.), 2006, Sujets, activités, environnements. Approches transverses. PUF. Éducation et formation. France.

Barbier J.M. (dir.), 1998, Action, affects et transformation de soi. PUF. Éducation et formation. France.

Barrow C. W., 1990 Universities and the Capitalist State: Corporate Liberalism and the Reconstruction of American Higher Education, 1894-1928. Madison, WI: University of Wisconsin Press. USA. 
Barth F., 2002, «An Anthropology of knowledge », Current anthropology, vol. 43, n 1, p. 111.

Barthes R., 2002 [1957], «Mythologiques », Euvres Complètes tome I. p. 671-870. Le Seuil, Paris.

Bateson G., 1977, Vers une écologie de l'esprit, Éditions du Seuil. Paris.

Becker G., 1984, Human capital: a theoretical and empirical analysis, with special reference to Education, $3^{\text {rd }}$ edition, University of Chicago Press. USA.

Béguin-Verbrugge A., 2009, «Information, Communication et Anthropologie des savoirs » Electronic Journal of communication information and innovation in Health, Rio de Janeiro, v. 3, $\mathrm{n}^{\circ} 3$. ISSN 1981-6278.

Berger P., Luckmann T., 2012, La construction sociale de la réalité, Armand Colin. Hors collection. France.

Berrou C., Gripon V., 2012, Petite mathématiques du cerveau. Une théorie de l'information mentale. Odile Jacob. France.

Bertaux D., 1996, Les récits de vie : perspective ethnosociologique, Nathan. 128. Paris.

Bertrand I., 2003, «Les dispositifs de FOAD dans les établissements d'enseignement supérieur : transfert ou intégration ?», Distances et savoirs 1 (Vol. 1), p. 61-78. France.

Blondel M., 2013 (1893), L'action, PUF. Quadrige. France.

Bloor D., 1976, Knowledge and social imagery, Chicago University Press. USA.

Boltanski L., 1982, Les cadres. La formation d'un groupe social, Éditions de Minuit. Le sens commun. Paris.

Bortef G. le, 2009, l'article « Ingénierie de la formation » dans l'Encyclopédie de la formation (Puf-Demos) dirigée par Barbier, Bourgeois, Chapelle et Juano-Borbalan. PUF. France.

Bortef G. le, 1990, L'ingénierie et l'évaluation de la formation, Les Éditions d'Organisation. Paris.

Le Boterf G., 2011, «L'ingénierie de la formation: quelles définitions et quelles évolutions ?», in Carré P., Caspar P., Traité des sciences et techniques de la formation, p. 383-400. Dunod. France.

Bourdieu, P., Lauretta C., 1996, The State Nobility: Elite Schools in the Field of Power. Stanford University Press.

Bourdieu P., 1986, «L'illusion biographique », Actes de la recherche en sciences sociales, numéro 62/63, Éditions de Minuit. Paris.

Bourdieu P., 1984, Homo Academicus, Éditions de Minuit. Le sens commun. Paris. 
Bourdieu P., 1980, Le sens pratiques. Éditions de Minuit. Paris.

Bourdieu P., 1979, La distinction, Éditions de Minuit. Le sens commun. Paris.

Bourdieu P., 1972, Esquisse d'une théorie de la pratique, Droz. Genève.

Bourdieu P., Passeron J.C., 1970, La reproduction. Éléments pour une théorie du système d'enseignement, Éditions de Minuit. Paris.

Boutinet J.P., 2010, Grammaire des conduits à projets, PUF. Collection Formation et pratiques professionnelles. France

Bouveresse J., 2007, Peut-on ne pas croire ? Sur la vérité, la croyance et la foi, Agone. Banc d'Essai. France

Braudel F., 2013, Écrits sur l'histoire, Flammarion. Champs Essais. France

Brousseau G., 1980, «L'échec et le contrat », Recherches, 41, p.177-182.

Bruillard E., Baron G-L., 2009, « Travail et apprentissage collaboratifs dans l'enseignement supérieur : opinions, réalités et perspectives », Quaderni [En ligne], 69 | Printemps 2009, mis en ligne le 05 avril 2012, consulté le 31 janvier 2017. URL: http://quaderni.revues.org/327 ; DOI : 10.4000/quaderni.327

Canaan, Joyce E., Wesley S., 2008 Structure and Agency in the Neoliberal University, Routledge. New York.

Carnevale A.P., Schultz E.R., 1990, «Return on Investment: Accounting for Training », Training and Development Journal, V44 n7 pS1-S32 July 1990.

Caron P.A., Becerril-Ortega R., Réthore S., 2010, « Modèle artisanal de la formation à distance », Conférence JOCAIR 2010. Amiens. France.

Cathelat B., 1990, Socio-styles système. Les styles de vie : théories, méthodes, applications, Éditions d'Organisation. France

Charle C., Verger J., 2012, Histoire des universités XII ème XXI ème siècle, PUF, Quadrige manuel. France

Cheng, Chau, 2012, «A study of the effects of goal orientation on the reflective ability of electronic portfolio users », Internet and Higher Education $\mathrm{n}^{\circ} 16$.

Chevallard Y., 1992, «Concepts fondamentaux de la didactique : perspectives apportées par une approche anthropologique », Recherches en didactique des mathématiques, vol. 12 no 1 , 73-111.

Cisel M., 2005, Réponses aux détracteurs de l'enseignement en ligne, in Cités, 2015/3, №63, PUF. France. 
Collectif, 2006, Visionary Observers. Anthropological Inquiry and Education, University of Nebraska Press. Lincoln and London. USA.

Cohen E., 2010, “Anthropology of Knowledge" Journal of the Royal Anthropological Institute.

Coulon A, 1993, Ethnométhodologie et education, PUF, L’éducateur. Paris.

Crozier M., Friedberg E., 1974, L'acteur et le système, les containtes de l'action collective, Points Essais. France.

Danvers, F. (dir.),2003, Anthropologie de l'éducation et de la formation, SPIRALE $n^{\circ} 31$. France.

Dearborn G., Louise S., Fifty Years of Anthropology and Education, 1950-2000: A Spindler Anthology, Publisher Lawrence Erlbaum Associates.

Delpech Q., Diagne M., 2016, Note d'analyse : «Mooc français, l'heure des choix», www.strategie.gouv.fr. France.

Demaizière F., Cord-Maunoury B., 2003, «Penser une formation aux TIC. Une professionnalisation des acteurs de la formation : formateurs et chefs de projet $»$, Distances et savoirs 4/2003 (Vol. 1), p. 533-550 : www.cairn.info/revue-distances-et-savoirs-2003-4-page533.htm.

Descola P., 2005, Par-delà nature et culture, Gallimard. Paris.

Descola P., 1986, La nature domestique. Symbolisme et praxis dans l'écologie des Achuar, Éditions de la maison des sciences de l'homme. Paris.

Desrosières A., 2010, Politiques des grands nombres. Histoire de la raison statistique, La découverte. France.

Detienne M., Vernant J.P., 1974, Les ruses de l'intelligence. La mètis des Grecs. Flammarion, Paris.

Derrida J., 2001, L'université sans condition, Galilée. Incises. Paris.

Develay M., Godinet H., Ciekanski M., 2006, «Pour une écologie de la responsabilité pédagogique en e-formation. », Distances et savoirs 1/2006 (Vol. 4), p.6172 : www.cairn.info/revue-distances-et-savoirs-2006-1-page-61.htm.

Dewey J., 2011, La formation des valeurs, traduction d'A. Bidet, La découverte. Les empêcheurs de penser en rond. France.

Dewey J., 2004, Democracy and education, Dover Publication.

Dodge B., 2011 (1961), Al Azhar, one millenium of muslim learning, Literacy Licensing, The middle east institute. 
Duhem P., 2007, La théorie physique, son objet, sa structure, Vrin. Bibliothèque des textes philosophiques. France

Durkheim E., 1975 [1910], «Le problème sociologique de la connaissance » dans Textes 1 : Eléments de théorie sociale, p190-194. Éditions de Minuit. Paris.

Durkheim E., 1938, L'évolution pédagogique en France, Paris.

Estellat N., Leroux E., 2011, «Le rôle des commerciaux dans le développement de la performance organisationnelle: le cas des CFC dans les établissements d'enseignement public » Revue Management et Avenir, juin 2011, Issue 45, p.144-155.

Fabre M., 1994, Penser la formation, PUF. L'éducateur. France.

Flamant N., 2002, Une anthropologie des managers, PUF, Col. Sciences sociales et société. France.

Ferrandis Y., 2013, La Raep dans les concours, La documentation française. France.

Foucault M., 2001, Dits et écrits 2 1976-1988, Gallimard. Paris.

Foucault M., 1975, Surveiller et punir ; Gallimard Tel. Paris.

Foucault M., 1966, Les mots et les choses, Gallimard Tel. Paris.

Friedberg E., 1997, Le pouvoir et la règle, Seuil. Points Essais. France

Garfinkel H., 1967, Studies in ethnomethodology, Englewoods, Cliffs, Prentice-Hall.

Geertz C., 1986, Savoir local, savoir global, Les lieux du savoir. PUF. Paris.

Gilli P., Verger J., Le Blévec D., 2007, les universités et la ville au Moyen-âge. Cohabitation et tension. Leiden, Boston.

Godelier M., 1974, Un domaine contesté : l'anthropologie économique, Mouton. France.

Goffman E., 1991, Les cadres de l'expérience, Les éditions de Minuit, Le sens commun. France.

Goffman E., 1990, The presentation of self in everyday life, Penguin.

Goody J., 1979, La raison graphique, la domestication de la pensée sauvage, Éditions de Minuit. Paris.

Gueudet, G., Lameul, G., Trouche L., 2011, «Questions relatives à la 'pédagogie universitaire numérique'. Regard et rôle de la recherche ». Revue Internationale des Technologies en Pédagogie Universitaire (RITPU), 8(1-2).

Gusdorf G., 1964, L'université en question, Payot. France. 
Hacking I., 2000, The Social Construction of What? Harvard University Press. USA.

Halbawchs M., 1994, Les cadres sociaux de la mémoire, Éditions Albin Michel. Collection l'évolution de l'humanité. France.

Hall E.T., 2014, La dimension cachée, Seuil. Points Essais. France.

Hall E.T., 1992, La danse de la vie, temps culturel et temps vécu, Seuil, Points Essais. France.

Hammersley M, Traianou A., 2012, Ethics in qualitative research. Controversies and contexts, Sage. Londres.

Hammersley M., Atkinson P., 2007 (third edition), Ethnography, principles in practice, Routledge. UK.

Hayek F.V., 2013, Droit, législation et liberté, PUF. France.

Hayek F. V., 1986, Scientisme et sciences sociales, Pocket. France.

Heidegger M., 1986, Etre et temps, Gallimard. Bibliothèque de philosophie. France.

Heidegger M., 1971 (1961), Nietzsche, Gallimard. Bibliothèque de philosophie. France.

Hofestede, 2010, Cultures and organizations, software of the mind, McGraw-Hill Professional.

Holland, Dorothy C., Margaret A. Eisenhart, 1990 Educated in Romance: Women, Achievement, and College Culture. Chicago, IL : University of Chicago Press.

Hryshchuk S., 2005, «La trilogie « coût-population-qualité » appliquée au tutorat à grande échelle. », Distances et savoirs 2/2005 (Vol. 3), p. 133-156 : www.cairn.info/revue-distanceset-savoirs-2005-2-page-133.htm.

D'Iribardne P., 1993, La logique de l'honneur, gestion des entreprises et cultures nationales, Seuil. Points Essais. France.

Jacob C., 2011, Pour une anthropologie historique des savoirs. Conférence présentée le 4 février 2011 devant la Société française pour l'Histoire des Sciences de l'Homme. Paris.

Jankélévitch V., 2011, L’irréversible et la nostalgie, Flammarion. Champs Essais. France.

Jézégou A, 2012, «La présence en e-learning : modèle théorique et perspectives pour la recherche », Journal of Distance Education / Revue de l'Education à Distance, 26(1). France.

Jézégou A, 2011, «Se former à distance : regard sur les stratégies d'autorégulation environnementale d'étudiants adultes », Savoirs, Revue Internationale de Recherches en Education et Formation d'Adultes, 24, 79 - 99.

Jorro A., 2007, Évaluation et développement professionnel, L’Harmattan. Paris.

Koyré A., 1988, Du monde clos à l'univers infini, Gallimard. Tel. Paris. 
Koyré A., 1985, Études d'histoire de la pensée scientifique, Gallimard. Tel. Paris.

Kuhn T, 1983 [1962], La structure des révolutions scientifiques, Flammarion. Paris.

Lameul G., Loisy C., 2014, La pédagogie universitaire à l'heure du numérique, (préface De Bernadette Charlier), De Boeck Supérieur. Belgique.

Lameul, G., 2008, «Les effets de l'usage des technologies d'information et de communication en formation d'enseignants sur la construction des postures professionnelles », Savoirs, 17, 73-94.

Latour B. Woolgar S., 2005, La vie de laboratoire, la production des faits scientifiques, Éditions la Découverte. France.

Latour B., 1989, La science en action, la Découverte. France

Lave J., Wenger E., 1991, Situated Learning, Legitimate peripheral participation, UK.

Lebon G., 1920, Psychologie de l'éducation, Édition Kindle de Flammarion.

Levi Strauss C., 2008 [1962], La pensée sauvage, in Euvres, p553-872, Gallimard. Pléiade. Paris.

Levi Strauss C., 1987, Race et histoire, Denoël Folio. Paris.

Levi Strauss C., 1958, Anthropologie structurale, Plon Pocket. Paris.

Loiret P-J., 2009, «Politiques publiques et stratégies des acteurs. », Distances et savoirs 4/2009 (Vol. 7), p. 645-666 :www.cairn.info/revue-distances-et-savoirs-2009-4-page645.htm.

Lopez-Fernandez et Rodriguez-Illera, 2008, « Investigating university students' adaptation to a digital learner course portfolio » in Computers \& Education $\mathrm{n}^{\circ} 52$.

Loisy C, Lameul G., 2015, «Les universités à l'heure de la pédagogie numérique ». Questions de pedagogies dans l'enseignement superieur (QPES), Jun 2015, Brest, France. Actes du VIIIe colloque Questions de Pedagogie dans l'Enseignement Superieur, <http://www.colloque-pedagogie.org/? $<$ hal-01326640> .

Marcel JF, Picot T., 2014, «Le travail collectif des enseignants en question(s) », Questions vives, recherches en éducation, $\mathrm{n}^{\circ} 21$.

Martinez, ML., 2004, coordination de deux numéros ( $\mathrm{N}^{\circ} 23$ et $\left.\mathrm{N}^{\circ} 24\right)$ de la Revue TREMA consacrés à « Approche (s) anthropologique (s) en éducation et en formation ».

Marty O, 2016,. «Licence ouverte. Utilisation des Cloms pour obtenir le grade de licence ». [Rapport Technique] Direction générale de l'enseignement supérieur et de l'insertion professionnelle, Ministère d'enseignement supérieur et de la recherche. 〈halshs-01329191>. France. 
Marty O., 2016, «Penser l'enseignement à distance. Valeurs historiques, économiques et esthétiques d'un nouvel élitisme », Journal of distance education, Athabasca University, Vol $32, n^{\circ} 1$, Canada.

Marty O., 2015, «Un modèle d'ingénierie coopérative. Contribution à l'ethnologie des opérations conjointes : l'algèbre du moment ». Biennale de l'éducation, de la formation et des pratiques professionnelles 2015 : "Coopérer ?", Jun 2015, Paris. <halshs-01181335>

Marty O., 2015, «Du métier informatique à l'art de la formation. Une critique épistémologique et esthétique du didacticiel Ammonite». Chapitre de : Cardona Gil Emmanuel, Lemaître Denis (dir.). La modélisation des activités managériales au défi de la formation. Analyse d'un serious game, L'Harmattan, Collection Action et savoir, p. 75-92, 2015, 978-2-343-06389-8. France.

Marty O, 2015, An anthropology of education management: Out of socio-political field studies of adult education conception. Document de travail en ligne : <halshs-01233789>.

Marty O., 2015, «A model of distance analysis. Epistemic field notes for education ethnographers ». Ethnography and Education, vol 15, Issue 1, p.17-27, Taylor and Francis Routledge, UK.

Marty O., 2014, «Monetizing French distance education. An enquiry on higher education value(s) », International Review of Research On Distance Learning, avril, vol 15, n², Canada.

Marty, O. (dir.), 2013, dossier : « Théâtre et formation » dans Education Permanente, ${ }^{\circ} 193$. Paris.

Marty O., 2013, «Lumières sur l'improvisation théâtrale : trois usages éducatifs d'une pratique sociale innovante ». Éducation permanente, Documentation française, p. 59-68. <halshs-00806538>, France.

Marty O., 2013, «Les métiers innovants de la formation. Le champ de la formation professionnelle et l'ingénierie de formation à distance». Les métiers innovants de la formation, France. $\leq$ halshs-01007448>

Marty O, 2013. «Learning on an online campus. Students team building through online interactions with a LMS ». ICERI2013. 6th International Conference of Education Research and Innovation, Spain. 〈halshs-00923472>

Marty O., 2012, «Le 'projet G.' : une ingénierie de formation. Conception et gestion d'un dispositif d'enseignement (le geste et l'effet). » Document de travail pour l'Axe 4 Organisations et formation du CRF. <halshs-00776590>. France. 
Marty O., Moreau R., Weinberger D., 2004, Start Up ? Du mythe médiatique aux réalités sociologiques, L'harmattan, Dossier de sciences humaines et sociales, Paris.

Marty O., 2004, Klikoo.com, ethnographie d'une start up, Éditions Le Manuscrit. France.

Marty O., 2003, Le son et le sens. De la musique dans les mots, Éditions Le Manuscrit. France.

Marty O., 2002, «À partir de la construction d'une pirogue Djuka : milieu fermé versus milieu ouvert ». ethnographiques.org, Numéro 2 - novembre 2002. France.

Marty O., 2002, « La vie de Start Up » dans Gérer et comprendre, mars 2002, n67, Revue de l'École des Mines de Paris en ligne sur http://www.annales.org/gc/2002/gc67-2002/marty004015.pdf. Paris.

Marty O., 2001, «Fins et moyens dans le processus institutionnel. Comment les institutions s'émancipent de leur projet initial ». Labyrinthe. Atelier interdisciplinaire, Hermann, 2001, p. 105-107. $\leq 10.4000 /$ labyrinthe.1122>. <halshs-01261126>. Paris.

Marx K., 2008 [1867], Le capital. Critique de l'économie politique, Livre 1 : le procès de production du capital, Folio Essais. France.

Mauss M., 2002, Manuel d'ethnographie, Petite bibliothèque Payot. France.

Mialaret G., 2006, Sciences de l'éducation : aspects historiques, problèmes épistémologiques, PUF. Quadrige Grands Textes. France.

Mir, Shabana, 2009, «Not too 'College-Like,' Not too Normal: American Muslim Undergraduate Women's Gendered Discourses. »Anthropology \& Education Quarterly, 40(3), p. 237-256.

Mises L. Von, 2014 [1921], L'action humaine, Traité d'économie. Les belles lettres. Paris.

Moeglin P., 2010, Les industries éducatives, PUF. Que sais-je ? France.

Morin E., 1986, La méthode. 3 : la connaissance de la connaissance, Seuils. Points Essais. Paris.

Moore M. G., 2007, «The Theory of Transactional Distance » in Handbook of Distance Education, p. 89-105, Second Edition, Lawrence Erlabum Associates, publishers. Mahwah, New Jersey, London.

Moreno J.L., 1987, Psychothérapie de groupe et psychodrame, PUF. Quadrige. France.

Musselin C., Kehm B., 2013, The development of higher education research in Europe. 25 years of CHER. Sense Publisher, Rotterdam. 
Musselin C., Neave G., Amaral A., Maassen P., 2009, European Integration and the governance of higher education and research, Springer.

Parménide, 1998, Sur la nature ou sur l'étant, Seuil. Points Essais. France.

Peraya D., 2016, «Des universités se mettent à la distance », Distances et médiations des savoirs[En ligne], 16|2016, mis en ligne le 17 janvier 2017, consulté le 28 janvier 2017. URL : http://dms.revues.org/1639.

Perriault J., 1989, La logique de l'usage - Essai sur les machines à communiquer, Flammarion. Paris.

Popper Karl, 1973, La logique de la découverte scientifique, Payot. Paris.

Poteaux N., 2013, «Pédagogie de l'enseignement supérieur en France: état de la question », Distances et médiations des savoirs [En ligne], 4 | 2013, mis en ligne le 08 octobre 2013, consulté le 08 février 2016. URL :http://dms.revues.org/403.

Pottiez J., 2011, Evaluation de la performance de la formation en entreprise par une approche systémique, Thèse de doctorat en stratégie et management des organisations. Université de Lille 1. France.

Rabardel P., 1995, Les hommes et les technologies, une approche cognitive des instruments contemporains. Armand Colin. Paris.

Reboul O., 2010, Qu'est-ce qu'apprendre? PUF, Éducation et Formation. France.

Renaut A., 2015, Les Révolutions de l'université. Essai sur la modernisation de la culture, Calman Levy. France.

Ricoeur P., 1991, Temps et récit, L'intrigue et le récit historique tome1, Points Essais. France.

Rommer T., 2014, L'invention de Dieu, Seuil. Les livres du nouveau monde. France.

Rorty R., Engel P., 2005, À quoi bon la vérité ? Gasset. Paris.

Rumble G., 2003, «Modeling the Costs and Economics of Distance Education » in Moore G.M. Handbook of distance education, pp703-717, Pennsylvania State University William G. Anderson Massey. University Mahwah. New Jersey, London.

Sauvé L., 2014, «Des dispositifs en ligne pour personnaliser l'apprentissage tout au long de la vie : quelques recommandations », Distances et médiations des savoirs [En ligne], 5 | 2014, mis en ligne le 26 février 2014, consulté le 17 juin 2014. URL : http://dms.revues.org/629

Shumar, W., 1997 College For Sale: A Critique of the Commodification of Higher Education. Falmer Press. Washington DC.

Simon G., 2012, Du mode d'existence des objets techniques, Aubier. Philosophie. France.

Sperber Dan, 1996, La contagion des idées, Odile Jacob. Paris. 
Stratégie nationale de l'enseignement supérieur et de la recherche (Stranes), disponible sur http://cache.media.enseignementsup-

recherche.gouv.fr/file/STRANES/12/2/STRANES_entier_bd_461122.pdf, (consulté en janvier 2017). France.

Stuart R., Long G., 1985, "Towards Marketing the Training Function, Part I : Adopting a Marketing Perspective", Personnel Review, Vol. 14, Iss : 2.

Thibault, F., 2007, Enjeux de l'enseignement à distance pour l'université française 19472004 (thèse de doctorat, Université Paris XIII - Paris Nord, France, sous la direction de Pierre Moeglin et Gaëtan Tremblay). Récupéré du site TEL (thèses-en-ligne) : http://tel. archivesouvertes.fr. France.

Unesco 2005, Vers les sociétés du savoir ? Rapport mondial. Éditions de l'Unesco. Paris.

Van Gennep A., 2011, Les rites de passage, A\&J Picard. Picard Histoire. France.

Vatin F., Callon M., Desrosières A., 2013, Evaluer et valoriser, une sociologie économique de la mesure, PUM. France.

Veblen T., 1979, Théorie de la classe de loisir, Gallimard. Tel. Paris.

Vernant J.P., 2007 [1965], «Mythe et Pensée chez les Grecs » dans Euvres p. 239-611, Éditions du Seuil. Paris.

Weber M., 1959 [1919], Le savant et le politique. Plon. Paris.

Witte A., 2010, Past and future culture. Booksurge Publishing.

Witthaud G., 2016, Validation of Non formal Mooc-based Learning, JRC Science for policy report, European Commission. UE.

Wittorski R., 2007, Professionnalisation et développement professionnel, L’Harmattan, Paris.

Wolff F., 1997, Dire le monde, PUF. Éssais Débats. France.

Wulf C., 1999, Anthropologie de l'éducation. L'Harmattan. Savoir et formation. France. 